M.

s 1. $x^{2}+x^{2}+x^{4}$

36)

Why

ase in

Y.t.

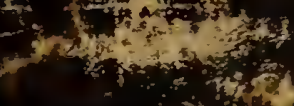

4.

(W. de

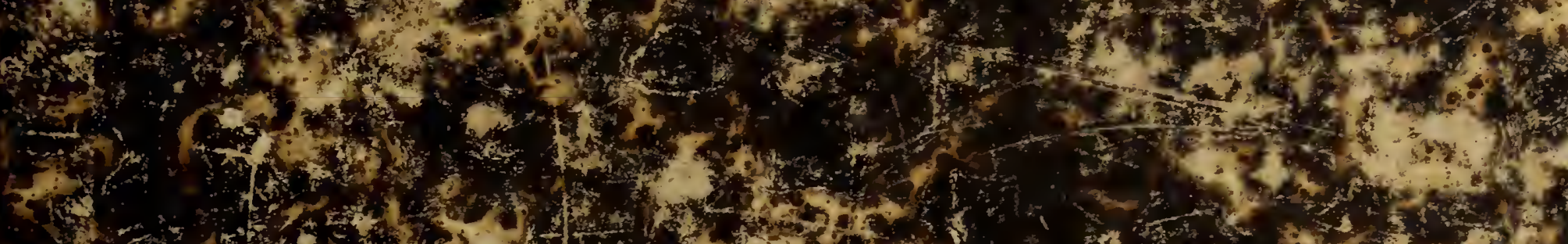

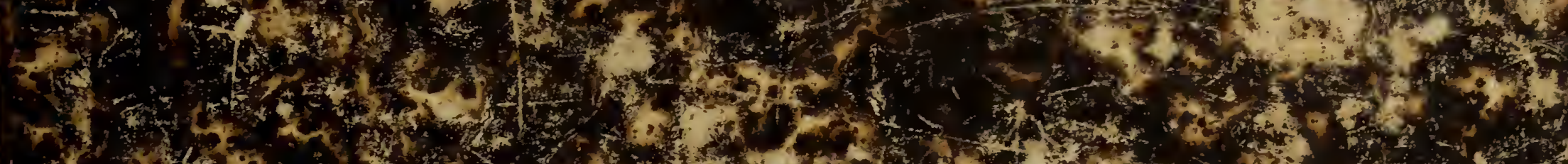
4.

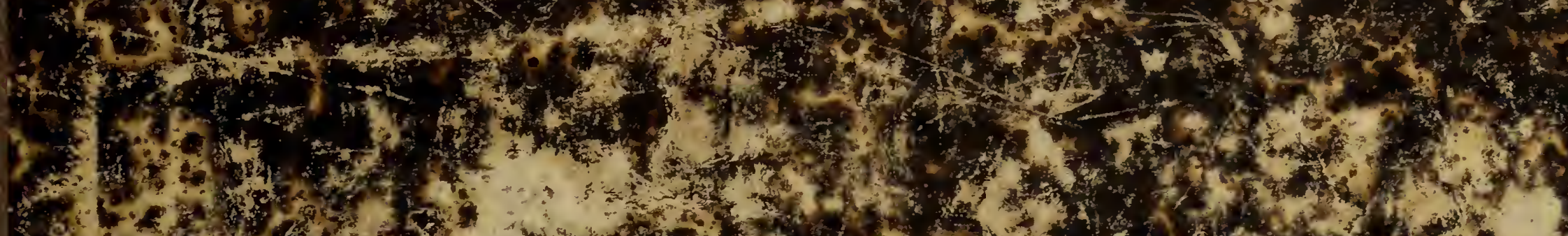

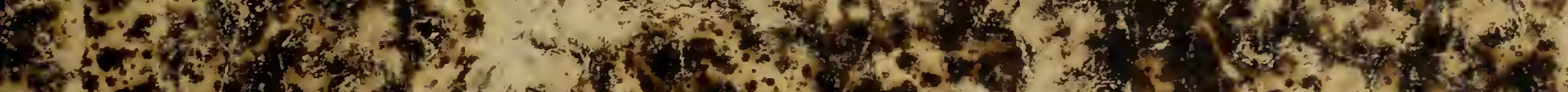

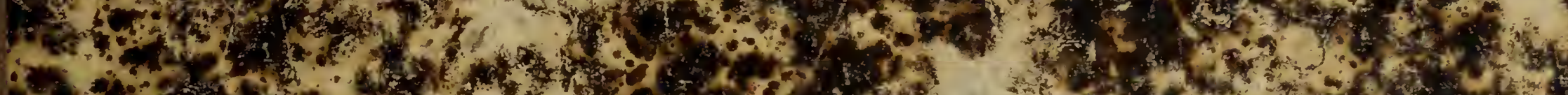

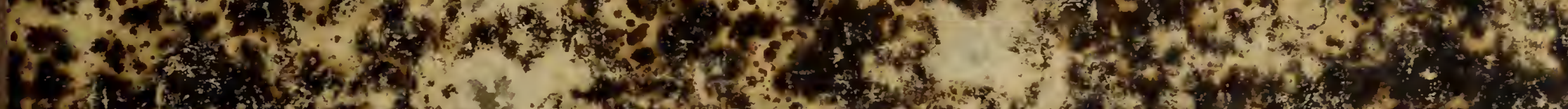

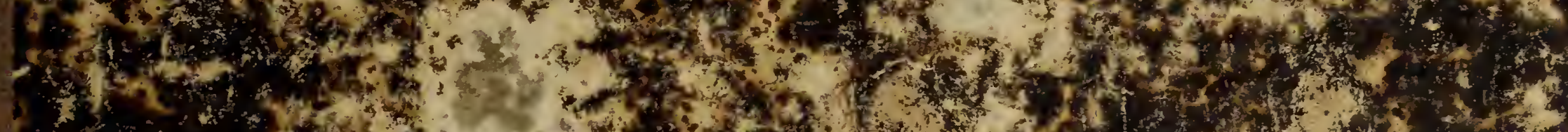

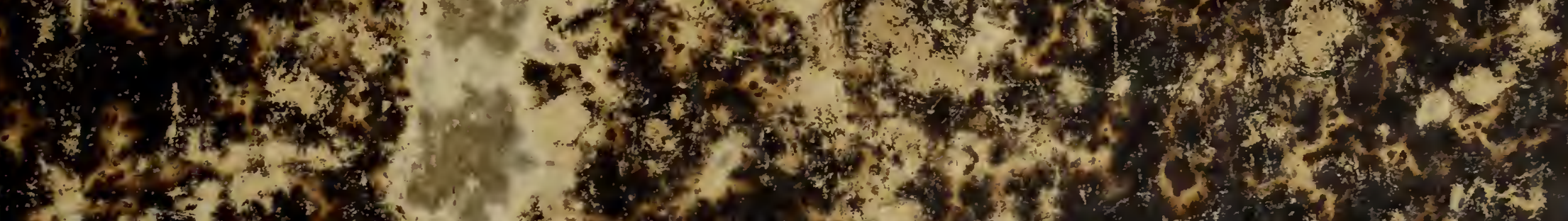

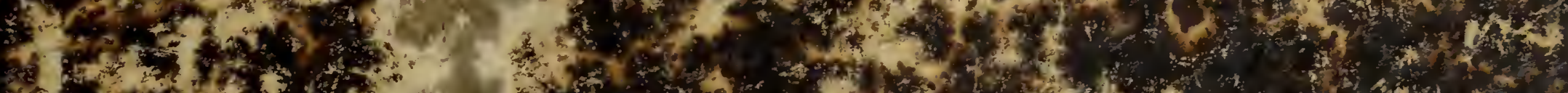

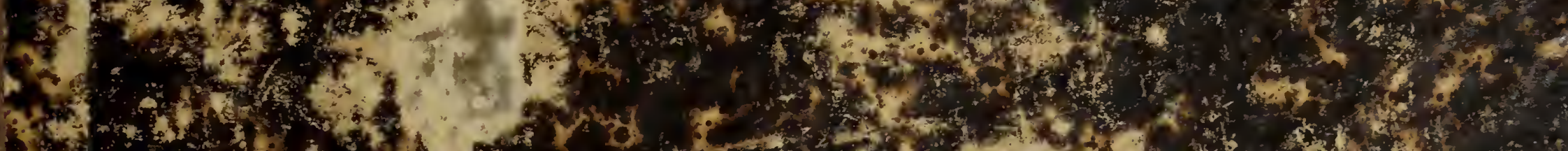
(3)

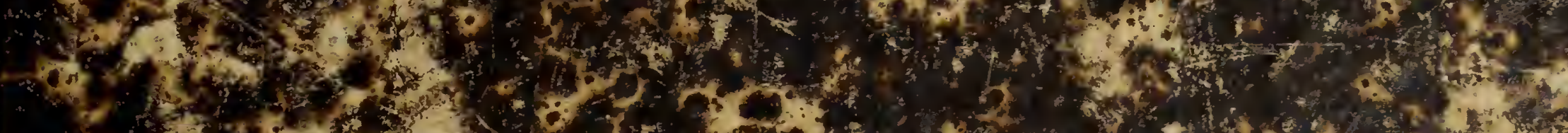

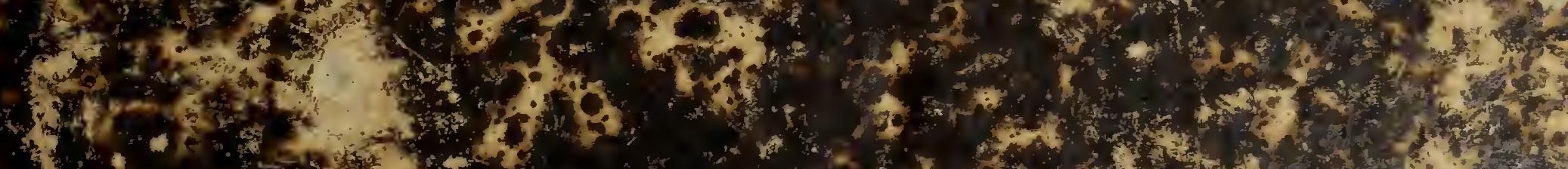

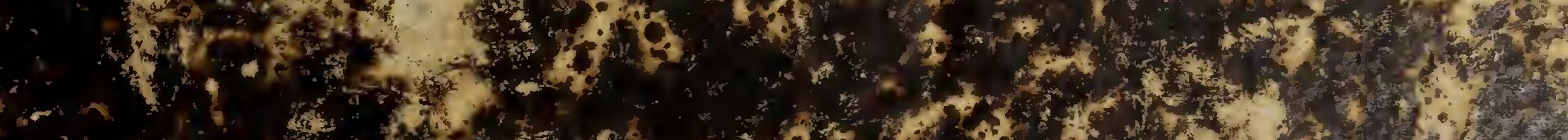

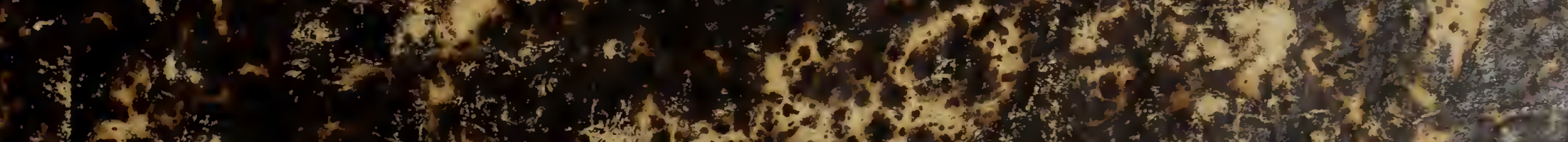

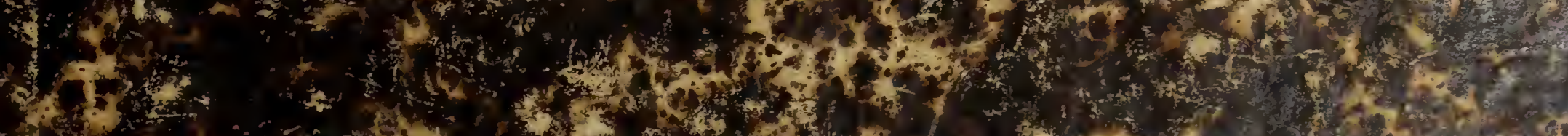

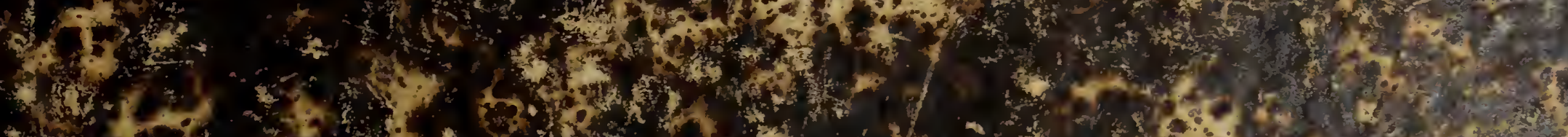

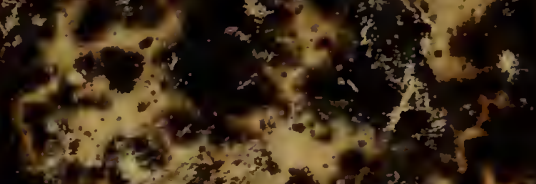


HARVARD UNIVERSITY.

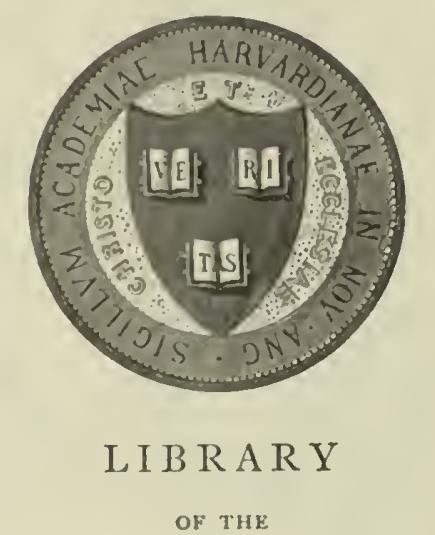

MUSEUM OF COMPARATIVE ZOÖLOGY $72,28,5$

LIBRARY OF SAMUEL GARMAN 



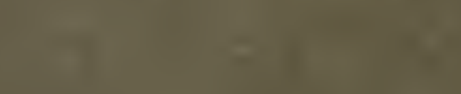
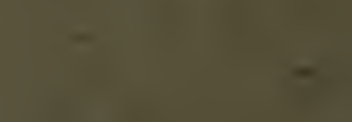

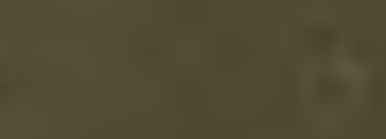






\section{DESCRIPTIONES ET ICONES}

$\begin{array}{lllllllllll}\text { A } & \text { M } & \text { P } & \text { H } & \text { I } & \text { B } & \text { I } & \text { O } & \text { R } & \text { U } & \text { M. }\end{array}$

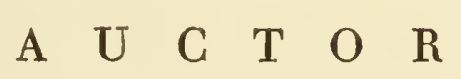

Dr. J O A N N E S $\underline{\underline{W}}$ A G L E R, NOR I M B E R G E N S IS.

$\operatorname{Tr} e s \quad p a r t e s$

c $u m$ X XXVI $\quad$ X $a b u l i s$.

MONACHII, STUTTGARTIAE ET TUBINGAE,

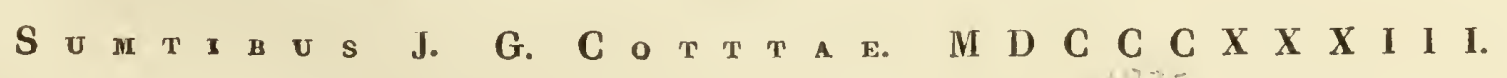





\section{$\begin{array}{llllllllll}\mathrm{P} & \mathrm{R} & \mathrm{O} & \mathrm{E} & \mathrm{M} & \mathrm{I} & \mathrm{U} & \mathrm{M} \text {. }\end{array}$}

Inter omnes Zoologiae studiosos constat, carere nos ad hoc usque tempus opere, in quo exhibeantur amphibia tam descriptionibus criticis, quam iconibus accuratis ornaț, quam ob rem ejusmodi operis editionem excusatione non egere mihi persuasum est.

Errores multiplices inde oriebantur, quod opus, quod complectatur Amphibia, examine quam diligentissimo nixum deficit, difficultas, qua hanc ob rem scientia ista impediebatur, plurimos deterrebat, ne campum huncce zoologiae uberrimum perscrutarentur. Hisce de causis descriptio Amphibiorum ex omnibus Zoologiae partibus una maxime neglecta, licet ipsâ maxime doceamur de organis inter se comparatis, quibus, ut ita dicam, vita et spontanei motus etiam superioris ordinis animalium efficiuntur.

Inductus juventutis ardore, qui, quae senectus timida fugit, suscipere amat, qui laboribus gaudet difficultatibusque ad id perficiendum, quod velit, qui denique naturae isti tam blandae conciliatrici se totum committit, simulque etiam incitatus laudibus, quibus Systema Avium illud a me editum est cumulatum et Amphibiorum Systema in medium proferre ausus sum. In hoc opere elaborando non temere collegi, quae jam alii conscripserunt, et pridem memorata iterum memorare fugi. Sed id solum ex auctoribus, qui me praecesserunt, retinui, quod natura ipsa erat comprobatum, et quod mihi naturae legibus consentire videbatur.

Hoc modo amphibiorum classificatio orta est, ab ea praecedentium auctorum dissimillima. Acerrimum meum oculorum visum utinam ingenii acumen adaequet! De hoc vero optime judicabunt, qui, ut ego, amphibii structuram tam internam quam externam contemplari solent.

Hoc novum Amphibiorum Systema, natum ex accurata consideratione atque contemplatione naturae, brevi in lucem edetur, idque hujus rei studiosis commendatum velim. Illud enim si respicis de generibus, quae hisce fasciculis nominatim tantum et speciatim exhibentur, judicare tibi licebit, quoniam illic argumenta sunt allata hujus rationis. De opere satis praefati sumus. Proferam ea nunc quae de isto edendo monenda habeo.

Wagler, Axphis. I. 
Hujus operis, quod inscribitur: „Descriptiones et Icones Amplibiorum" quarta quaque vel octara hebdomade unus fasciculus edetur duodecim pictis cum summa diligentia industriaque tabulis totidemque descriptionibus instructus. Ad hoc accedit, quod minime cum silentio praeterire possum, (unod, quae in hoc: opere continentur Amphibia, ad naturam ipsam sunt descripta, delineata et ad animal vivum depicta omnia; caque re maguum illi splendorem accessisse puto, quod illi quoque, qui naturam quasi animantem et virescentem malunt quam exsanguem et mortuam contemplari, non minimi pretii habebunt.

Delincatio vero ommiun, quae in hoc opere comprehendentur, Amphibiorum a me ipso est facta, quod singulae corporis partes et habitus ad constituendas species valde necessariae ab artifice non raro negligi soleant. - Ceterum in prioribus fasciculis praecipue genera exhibentur. Quod ad Amphibiorum mensuram attinct, monendum est, normâ Parisiensi in hoc me usum esse.

Monachii mense Februario

\section{IIDCCCXXVIII.}

JOANES WAgLER. 



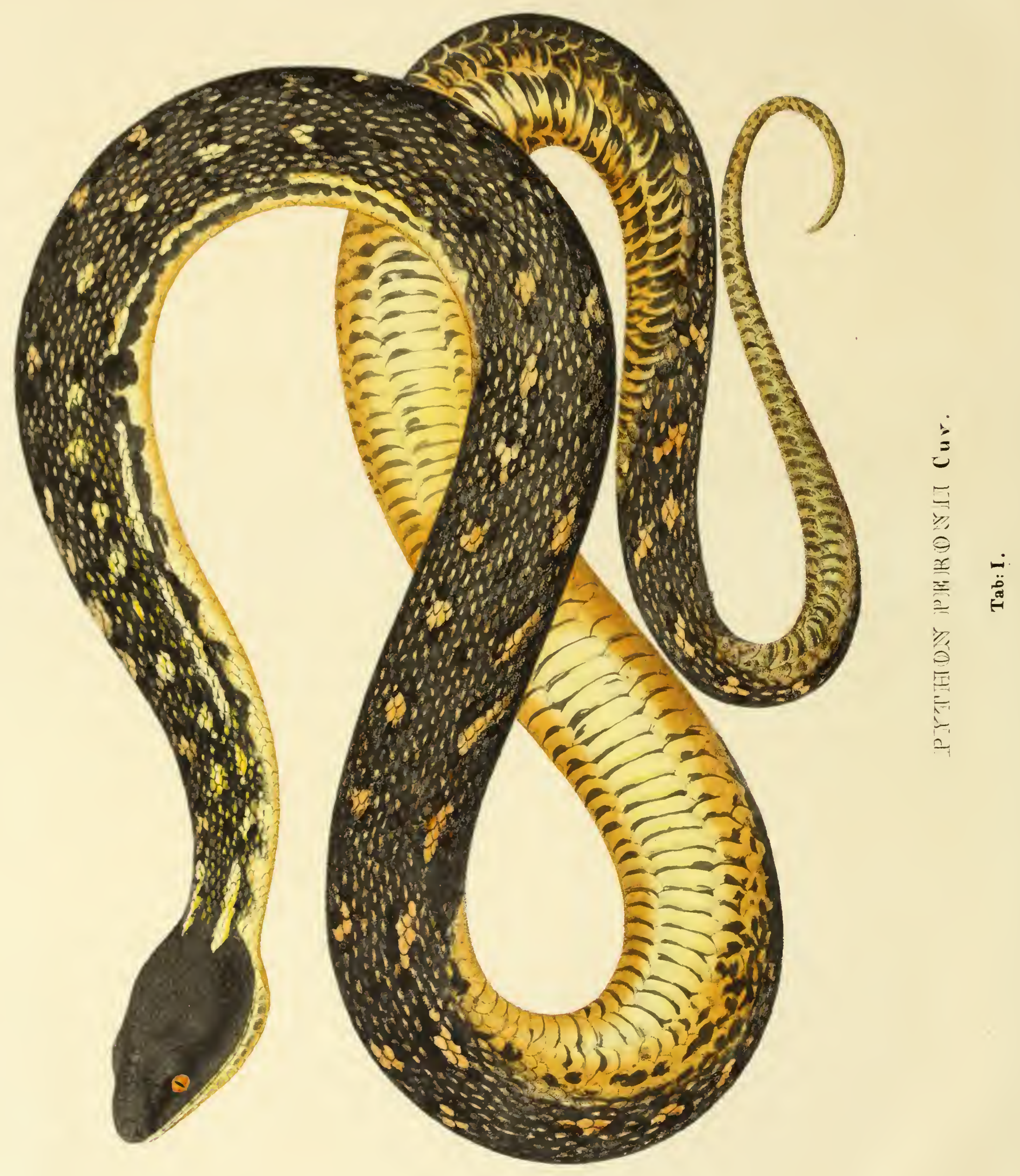




\section{PYTHON PERONII Cur.}

\section{$\begin{array}{lllllllllllllll}\mathbf{P} & \mathrm{E} & \mathrm{R} & \mathrm{O} & \mathrm{N} & \mathrm{S} & \mathrm{P} & \mathrm{Y} & \mathrm{T} & \mathrm{H} & \mathrm{O} & \mathrm{N} .\end{array}$}

T A B. I.

Character essentialis. Scutis frontalibus quatuor majusculis perquam convexis, capitts scutorum omnium maximis; scuto verticali minutissimo, squamulis subsequentibus irregularibus margine subsursum plicatis; scutis abdominalibus angustissimis, squamas in utroque latere adjacentes magnitudine parum superantibus; squamis trunci antici ovatis, postici rhomboideo-lanceolatis, omnibus laevibus; trunco toto et cauda supra nigricantibus, squama unaquaque puncto intermedio flavo, dorso antico praeterea medio hic et illic macula irregulari, parva, citreo-flava, lateraliter lineola, horizontali concolore notato; labiis, capite et trunco subtus flavis, trunco postico et cauda subtus nigricanti-variis. Scut. abdom. 276, scut. infracaud. 84-86.

Synonyma: Snaken. 5. White Journ. of a Voy. to New South Wales p. 259 (descriptio et figura adjecta pessimae); Australasian Snake Shaw Gen. Zool. 3. p. 505 (Descriptio manca); Python Peronii Cup. in Mus. Paris., Python punctatus Merr. Syst. Amphib. p. 90. (Descriptio Whiteiana.)

\section{E S C R I P 'T I O.}

Corporis forma universalis. Caput distinctum, mediocre, ovatum, depressum, vertice convexiusculum, prope rostrum acutiusculo-obtusatum, compressiusculum, oris angulus rectus; nares mediocres subtriangulari-ovales antrorsum spectantes; oculi laterales, mediocres, longiusculo-rotundi fere in medio capitis lateralis siti, unde a rostri apice perquam amoti; scuta: scuium rostrale latiusculum apice supremo lanceolato-acutissimo triangulum, antice medio convexiusculum; scuta frontalia anteriora et posteriora bina omnium capitis maxima, majuscula, medio, praesertim postica, perquanı convexa, anteriora, posterioribus magnitudine aequalia, trigona, apice antrorsum verso, pone rectiusculotruncata, posteriora rhomboidea; scutum verticale (multum ante oculos situm) minutissimum, fere lanceolatum scutellis duobus subsequentibus multo majoribus rhombeo-rotundatis, scuta occipitalia repraesentantibus, pone in medio aliud minus, subliexagonum includentibus; squamulae sequentes, forma irregulares, convexae et concavae; scuta minima, forma diversa inter utrumque oculum sita margine sursum flexa, subsequentibus aliis forma et magnitudine similibus, attamen planis sive obsolete convexis; scutella superciliaria, forma variantia, quatuor; scutorum ocularium posteriorum loco squamulae tres convexiusculae; regio a naribus usque ad oculos squamulis ovalibus, convexiusculis circiter 15 obsita; scuta marginalia labii superioris 12, parva, omnia quadrangula, versus oris angulum magis magisque altitudine decrescentia, convexiuscula; scuta marginalia labii inferioris 14, parva, a mandibulae apice versus oris angulum sensim majora, 5-6 postica quadrangula medio perquam excavata, reliqua integra, antica mediis longiora ac inferius subtrigono-terminata; scuti labialis ejusque scutorum accessoriorum loco squamae angustae, oblongae 5, integrae; caput totum inferius squamis ovato-lanceolatis obtectum, quae versus mandibulam reliquis paullo minores; dentes ut in reliquis Pythonibus formati, primores palatini reliquis dentibus vero longiores. Truncus elongatus, compressus, modice fusiformis, crassiusculus, abdomine subanguloso-rotundatus, superius medio squamarum seriebus 47; squa mae omnes laeves et versus scuta abdominalia magis magisque majora, dorsi antici minimae oblongo-ovales ac interdun subconcavae, dorsi medii ovatae, sensim rhomboideo-lanceolatae, serierum trium penultimarum in utroque latere magnae, latae, apice rotundato-acuminatac, seriei ultimae maximae, scutis abdominalibus similiter 
formatis paullo angustiotes, semilumlares, in ntoque vero apice introrsum abscissac; scuta abdomiu alia angustissima squamamn adjacentium forma, numerosa; scutum anale nediocre, $n$ ug u is extremitatis posticae ad utrumpene ani latus parvus, recurvatus; ca uda tenuis, brevinscula, contpressinsculoteres, sensim attenuata, apice acutiuscula, scntis inferioribns omnibus divisis, squamis subrhombeis superins obtecta. Scuta abdominalia 276 , scuta infracaudalia $84-86$.

Color. Caput totum supra, trunci caudaeque partes superiores et laterales omnes nigricant, inferiores fiatae sunt; uncha lineis duabus longitudinalibus, longiusculis, largiusculo interstitio distantibus, sulphureo-llavis notata; adjecta snnt infia has taenias horizontales lineolae, quae vix nsfpe ad tertian trunci partem conspicuae, ac nuchalibus colore similes sunt; squamac in hac tunci parte fere omnes nigricant, at in ea primum maculae substellilormes in duplici serie longitudinali collocatae, dein lineolae uansversae, maculis concolores, haud numerosae, sulphureo-flavac conspiciuntur, quoque jann omnes squanae, nltimis utrinque exceptis, in medio gerunt maculam flavan, sensin in squamis trunci inferioris ac caudae in aurantiun vergenten; nec lineoleac in trunen posteriore horizontales nec transversae adsmot, attanen maculae parvae in quadruplici serie, e maculis squamarum reliquis majoribus, tribus aut quatuor congestis, anrantiacis composita, magnis intervallis distant; flavedo squanas versus abdomen magis nagisque occupat et squanarum series uhina toto flavicat; scuta labialia maxillae et mandibulae, caput subtus nee non totum abdomen sulphureo-flava sunt et immaculata, exceptis scutis abdominalibus versus anum nugis magisque nigricanti-mubilatis; scuta infacaudalia flavo-nigricantique nubilata; irides ...?

Proportio corporis partium e mensura. Longitudo a rostri apice usque ad caudae

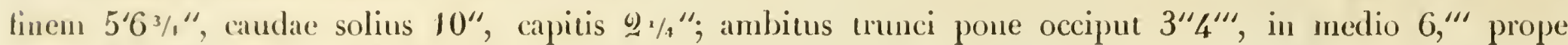

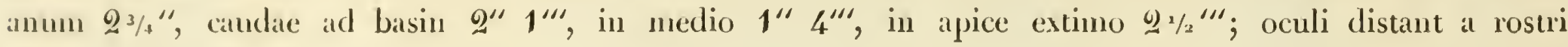
apice $7 \%$ \%".

Habitatio etvilae ralio. Habitat in Nova-Hollandia, non infrequens, ut videtur, in Dusk y-B a y.

OBSERVA'TIONES. Specimina hujus speciei adsunt in Museo Parisiensi, Lugdunensi Balav. ac in collectione mea; descripto vidi multo majora, allamen simili modo picla. Puncla nec non maculae trunci flava in speciminibus exsiccatis restant, at in ïs pallescunt quae in spirilu vini asservanlur. Species haec pulcherrima formal ob capilis structuram transitum a PYlhonibus ad Xiphosomala, allamen ad ex ea genus distinclum formandum characleres nullo modo sufficiunl. - Figuram Ithileianam in operis sui tabula 46 (Snake Nr. 1. p. 250.) spectandam juvenem PYlhonis Peronii monstrare credo. 



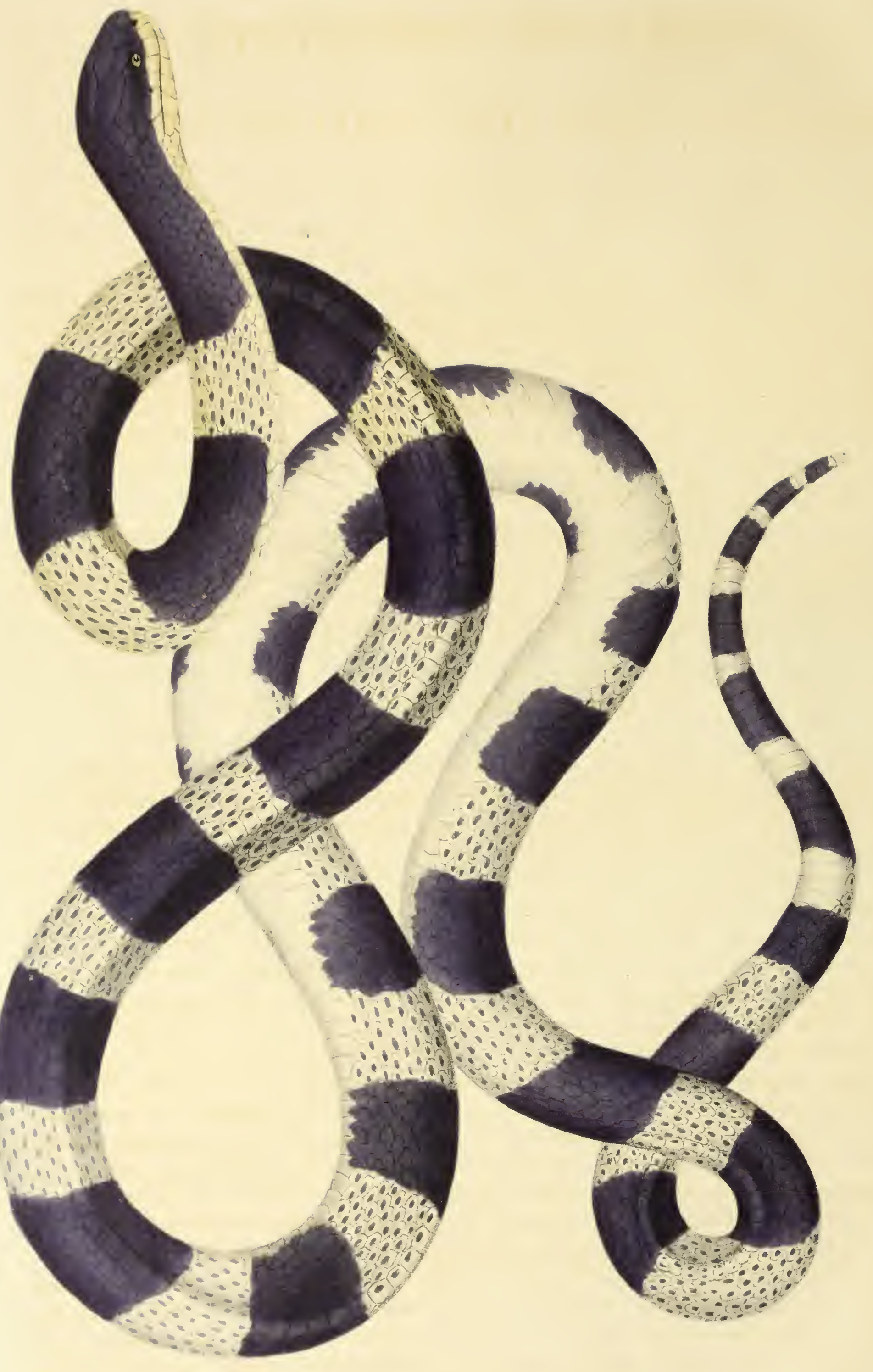

迹 


\title{
ASPIDOCLONION SEMIFASCIATUM.
}

\author{
H A L B GE B Ä N D E R T E R S C H I L D R Ü C K E N.
}

T A B. II.

Character essentialis. Squamis omnibus laevibus, trunci antici hexagonis postici majoribus subovato-rhombeis, caudae brevis, obtusiusculae rhombeis; spina dorsi ac caudae cestriformi-compressa; abdomine rotundato absque angulis prominulis, capitis parte infima labiisque laete flavis, unicoloribus; capitis partibus superioribus, occipite, nucha et collo supremo fasciisque trunci superioris circiter 20 latis, rectissimis, scutorum abdominalium marginem externum attingentibus violaceonigris, fasciis trunci totidem fere flavis, angustioribus et unaquaque squamarum macula parva violaceo-nigra medio notata; cauda annulis alternantibus violaceo-nigris, et flavis violaceo-nigro variegatis. Scut. abd. $212-220$, scut. infracaud. $46-50$.

Symonyin. Seba Thes. 2. t. 66. f. 1, 2, 3, 4 (figurae pessimae) - Coluber candidus Linn. Mus. Adolph. Frid. 1. p. 33. t. 7. f. 1. (Descriptio et icon non satis accuratae.) Bonnater. Ophiolog. p. 39. nr. 98. t. 21. f. 41. (icon Linneana.) Lacép., Latr., Daud. Bungarus semifasciatus Oppel in Mus. Paris., Boie in Mus. Lugd.

\section{E S C R I P T I O.}

Corporis forma unipersalis. Caput vix distinctum, depressissimo-ovatum, obtusum, latiusculum, ante oculos versus labia subrotundato-declive, impressiusculum, pone illos prominulo-convexiusculum, labiis convexiusculis, maxillae prominulis, rictu rectiusculo lato; faciei latae longitudo mediocris, nares rostri apici approximatae, in marginis interni apice scuti nasalis sitae, majusculae, perpendiculari-ovatae, deorsun spectantes, margine interno prominulo; oculi laterales, parvi, rotundi, pupilla circulari, parum prominuli, a rostri apice multum amoti; scuta: scutum rostrale magnum margine obsolete angulosum, subrotundatum, inferius medio emarginatum unde subsemilunulare; scuta frontalia antica duo mediocria, irregulariter quadrangula, latere externo subrotundato, scuta frontalia posteriora magna anterioribus dimidio majora pentagona, antice recto-, pone trigono-terminata; scutum verticale breve aeque longum ac latum, hexagonum, attamen angulus versus scuta frontalia posteriora spectans parum emergens; scuta occipitalia magna, irregulariter terminata, versus verticem triangularia, lateribus externis, subapicalibus obsolete biemarginata; scutum nasale magnum, quadrangulum, latere interno naribus emarginatum; scutum loreum mediocre, trigonum, cum apice acuminato versus oculos spectans, lateribus subtilissime emarginatum; scutum oculare anterius unicum mediocre, pentagonum, planum; scuta ocularia posteriora duo aequalis fere magnitudinis mediocria, superius quadrangulum, inferius, vix minus, subpentagonum; scuta superciliaria subparva, brevia, pone recto-truncata, bi-, antice tri-angulosa, plana, super oculos margine externo non proeminentia, sed ibi recta; scutum temporale unicum utrinque longiusculo-quadratum, interdum obsolete subquinquangulum, pone squamis nonnullis majusculis rhomboidalibus munitum; scuta marginalia labii superioris 7 , primum omnium minimum, triangulum, latere externo oblique truncatum, secundum, tertium et quartum aequali magnitudine et forma, mediocria, quinquangula, apice supremo triangula, lateribus rectissima, quintum majusculum praecedentium forma, attamen his dimidio majus ac latere inferiore quam superiore multo latius, sextum magnum, omnium maximum, pentagonum, superius perquam dilatatum et trigonum, versus basin magis magisque attenuatum, ultimum praecedente multo minus irregulariter quadrangulum, pone subrotundatum; scutum labiale medium mediocre, planum, trigonum, scuta labialia accessoria longiuscula apice interno regulariter triangula; scuta marginalia labii inferioris 6 , primum et ultimum omnium minima, parva, illud subre-

Wagen, Amphis. 1 . 
gulariter quadrangulum, hoc oblongo-quadratum, penultimum ejusdem formae, attamen duplo majus, sequens dein altius sed brevius, suboblique quadratum, proximum omnimn maximum, permagnum, scntiforme, margine inferiore usque ad scuta mentalia extensun, scutum sequens praecedente dimidio fere miuns, pone rotundatum, angustins versus prinum; scuta mentalia anteriora lata, posterioribus dimidio fere majora, harpiformia, posteriora mediocria, pone ovato-terminata, antice latere interno obliqne al)scissa; squamae gulares mediocres regulariter hexagonae; dentes pone tela imperforati 2-5, reliqui ut in omnibus speciebus hujus generis formati. 'Truncus elongatus gracilior, latere inferiore rotundatus, superiore versus spinam dorsi perqnam compressus, in spina dorsi ipsa acuminatus, aequali fere ambitu ab occipite usque ad anm, versus hunc et occiput paullo angnstior, per totam longitudinem squamarum seriebus 15, squam a omnes laeves, planae, trunci antici hexagonae, postici majores, subovato-rhombeae; scutella spinae dorsi lata, omnia sexangula, versus candam magnitudine paullo decrescentia, suprema medio perquam cestri-formi compressa; scuta abdominalia latitudine totum abdomen occupantia, numerosa, mediocria, scutum anale largum, pone rotundatum, integrum; ca uda brevis subtus planiuscula, reliqua parte tereti-compressiuscula apice subobtusa squamis fere rhombeis, spinae scutellis hexagonis, scutis inferioribus onmilous integris. Scuta abdominalia 212-220, scuta infracaudalia $16-50$.

Color. Capitis totius pars superior, nucha ac collum supremum fuliginoso-nigra, unicolora, egregie violaceo-resplendentia; trunci totius superioris pars reliqua fasciis alternantibus lacte flavis ac fuliginoso-nigro violaceis, latis; fasciae hac obscurae trunci supremi latissimae versus inferiorem magis magisque latitudine decrescentes, omnes versus scuta abdominalia, quorum latera attingunt, pallescentes; fasciae flavae obscuris semper nulto angustiores in squamis omnibus medio macula oblonga fuliginoso-nigro violacea, in scutellis maculis tahibus binis notatae, unde laete variae; cauda tota ejusdem picturae, attamen non fasciata, sed annulata, annulis binis obscuris, superius medio divisis, utplurimum subtus confluentibus; labia maxillae et mandibulae, capitis pars inferior ac reliqua trunci inferioris pars laete subvitellino-flava, immaculata; irides...?

Proportio corporis partium e mensura. Longitudo a rostri apice usque ad caudae finem 3' 5\%", caudae solius 4" 11" , capitis 1\%"; ambitus capitis immediate pone nares 1\%", pone oculos $1^{\prime \prime} 10 \% "$, occipitis $2 " 1^{\prime \prime \prime}$, colli supremi $13 / 4$, trunci in medio $22^{\prime \prime}$, prope anum 13/.", caudae ad basin 1" 4", in medio $11^{\prime \prime \prime}$, in apice extimo 5"'; oculi distant a rostri apice 4\%".".

Habitatio et vitae ratio. Habitat in $\mathrm{Java}$ insula satis frequens.

OBSERVATIONES. Exemplaria hujus speciei asservantur in Museo Parisiensi, Lugdunensi Batav., Monacensi ac in collectione mea; colore vix variant; quoque solum annuli flavi in spiritu vini albescunt. Huc sane referendus est LINNEI Coluber candidus cujus scutella spinae dorsalis describere omisit auctor clarissimus; LACÉPEDII ac DAUDINI adjecta ii vero varietas, mihi prorsus ignota, spectat colubrum toto coelo diversum. - Pone tela in osse maxillari dentes quinque imperforatos, parvos palatinis magnitudine aequales retrorsum flexos in speciminibus duobus a me dissectis observavi, duos solum invenit amicissimus HeNRICUs BoIE, igitur horum numerus non constans esse videtur. Pedis postici vestigium in specimine a me dissecto, male conservato, detegere mihi non contigit; forsan reperitur in exemplaribus melius adfectis. - Aspidoclonion annulare a hac specie primo intuitu, squamis trunci annulati latioribus, nec non capitis pictura distingui potest, specimina contra in museo Lugdunensi sub nomine Bungari Ferri equini KU H II spectanda, sicut hujus auctoris, descriptio ipsa nullam mihi ab Aspidoclonio annulari ostendebant, differentiam. 



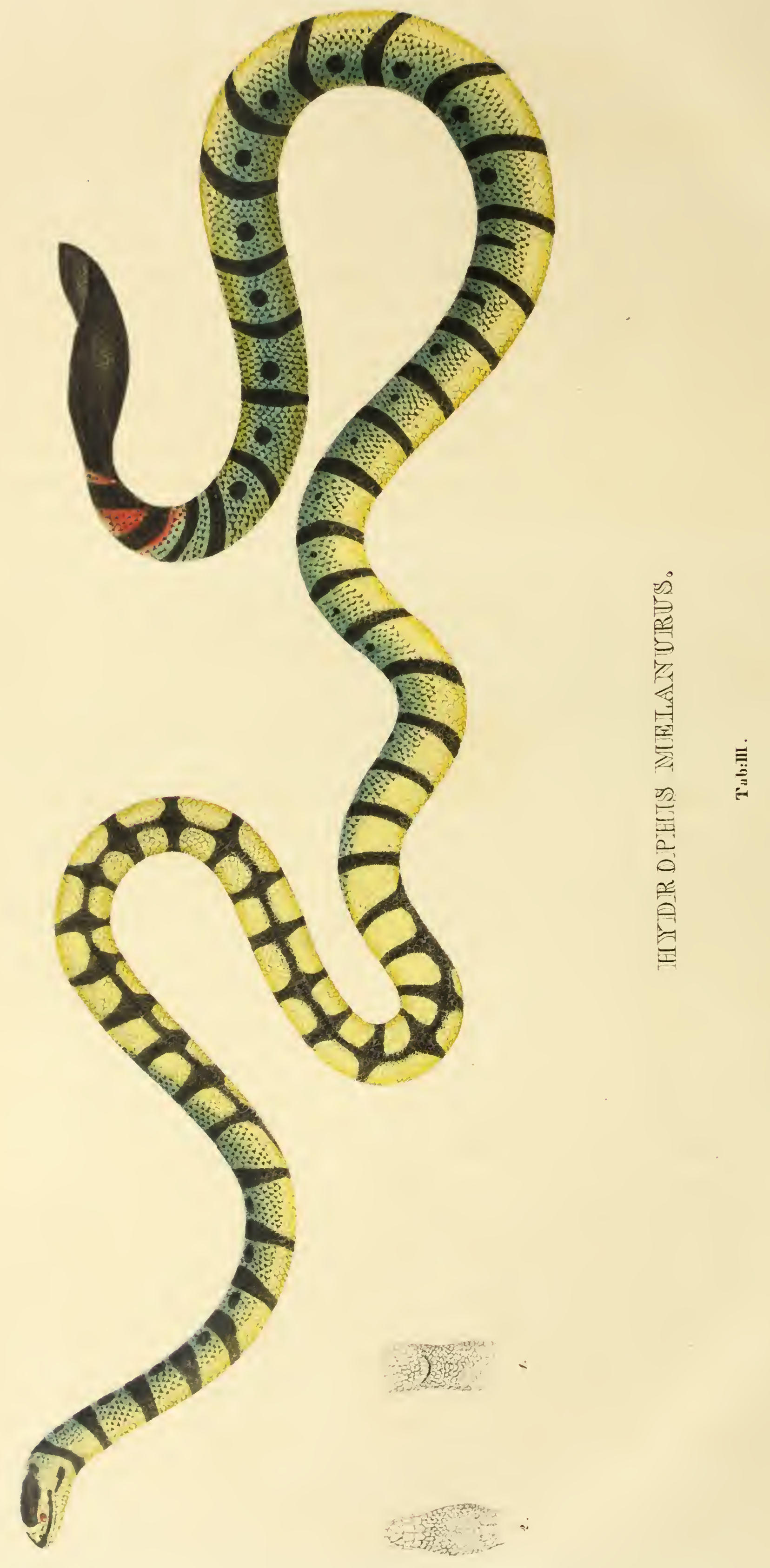




\title{
HYDROPHIS MELANURUS.
}

\section{SCHW ARZSCHW ÄNZIGE W ASSERSCHI,ANGE.}

\author{
T A B. III.
}

Cluaracter essentialis. Trunco gracili tereti-compresso, postico sensin latiore, superius in medio rotundato-compresso, squamis parvis rhombeis medio punctulo gibbo notatis; cauda Irunco non latiore trigono-terminata, schistaceo-aterrina, unicolore; scutellis abdominalibus hexasonis schistaceo-aterrinus, punctulis duobus aut tribus gibbis notatis; capite flavo exceptis taenia supra oris angulum orta, infra oculos dein arcuata supra rostrum infra nares ducta, macularue verticis usque ad occiput extensa nigricantibus; trunco supra laete olivaceo-viridi squamis nigricanti-marginatis, ad latera sulphureo-flavo ab occipite usque ad caudae basin fasciis in abdomine cam scutellis coadunatis, superius maculis rotundis schistaceo-atris interpositis variegatis dissecto; fasciis ante caudae basin duabus penultimis flavis rubro-lavatis. Scutellis abdominalibus 154.

SYNONYMIA: 0

\section{E S C R I P 'T I O.}

Corporis forma universalis. Caput indistinctum, oblongum, depressum, planum, obtusum, a naribus ad rostri apicem devexum, lateribus convexum; oris angulus subtiliter adscendens; mandibulae margo a maxillae illo inclusus; nares a rostri apice multum distantes, supernae, punctiCormi-rotundatae, exterius in scuti frontalis antici fissuram excurentes; oculi laterales altamen pilei margini proximi, perquam parvi, recti, circulares, pupilla circulari, parun prominuli, non scutorum superciliarium margine prosiliente adumbrati, oris angulo paullo propiores quam rostri apici; scuta: scutum rostrale majusculum, pentagonum, margine infimo subtiliter biemarginatum; scuta frontalia anteriora magna quadrata, in parte postica quam antica suboblique truncala paullo latiora; scuta frontalia posteriora anticis dimidio minora, pentagona; scutum verticale mediocre sublurriforme, margine antico subtiliter biemarginatum; scuta occipitalia magna, oblonga, margine externo subshliquo, rix anguloso, apice postico paullo attenuato, antico rectiusculo-truncato; scuta occipitalia serfuntur lateraliter squamae 2-3 reliquis trunci superioris nuajores, irregulares; scuta nasalia ac scuta lorea nulla; scutcllum oculare anticum unicum, parvum, regulariter pentagonum; scuta superciliaria latiuscula, freria, pone recto-truncata, antice obsoleto-trigona; scutum temporale unicum, magnum, rhombeo-pentagonum; scutella ocularia posteriora duo, quadrata, pone subrotundata; scuta marginalia labii superioris septenı, quorum ultimum squamiforne, penultimum ninutum, rhombeum, reliqua altissima, prima locum scuti lorei ac nasalis occupantia, versus narginem inferiorem convexa; omnium margo inferior aculus et a sequente interstitio parvo diremtus est; scutum primum mediocre, angustum, apice supremo subtrigonum, secundum omnium maximum apice supremo dilatatum, subtrigonum, tertium, quartum, quintun aequali altitudine ac latitudine, subobliqua, apice supremo subtrigona; scutum labiale medium subparrum, angustiusculum, acutotrigonum; scuta labialia accessoria majuscula, oblonga, lanceolato-terninata; scuta marginalia labii iuferioris octo, quorum duo ultima squaniformia, reliqua majuscula, tertium onnium maximum, onmia subrhombea, primum rero ac secuudum tertio paullo breviora sunt; quartum, quinto paullo majus, brevius est primo; scuta mentalia quatuor, majuscula, antica posticis dimidio majora, oblongo-sulsquadrata, postica fere pentagona; scuta omnia capitis obtecta sunt, accuratius contemplata, rerrncis minulissimis, uumerosis; gula obtegitur squamulis oblongo-rhombeis, subtilissime verruculosis; dentes ut in congeneribus formati, maxillae utrinque primus reliquis paullo major. Truncus longus, gracilior, ab occipite usque ad medium angustus, compressiusculo-teres, dein usque ad caudae basin sensim incrassatus, magis conpressus, supra et subtus in medio compresso-rotundatus; squamae parrae, trunci superioris medii rhombeae, lateralis hexagonae, omnes medio punctulo prominulo munitae; scutella abdominalia angustissima, hexigona, punctulis duobus aut tribus gibbis obtecta; scutella ante anum desinunt, quae re- 
gio squamis obtecta est pone rotmulatis subtilissime veruculosis; ani orificim semilunulare occurrit, margine postico spmammlis mmerosis, ingustis cinctum; canda hrevis, compressissina, munco postico non altior, subito trigono-teminata, in apice extimo squama magna, juxta quam latealiter utrinene alia trigona adest, terminata, squamis obsolete hexagonis, medio punculo gibbo armatis obsita; margo aaudac superior ac inferior rectissimms, integerrimus est, non pellucidus. Sculella abdominalia $15 / 4$.

Color: Caput flavum, macula lata a scutorum firontalium posticonm margine postico usque ad mucham extensa ac taenia supra oris angulum incipiens, infra oculos et arcuata ante nares supra rostrum ducta nigricantes; scutum labiale ac scuta mentalia olivascenti-lavata; dorsun totum laete olivascenti-viride squanamu limbo subtili nigricante, ejus latera ac abdonen lacte sulphmreo-flava, exceptis scutellormm abdominis serie schistaceo-atra ac lasciis ante caudam duabus penulimis flavis rubro-lavatis; canda tota schistaceo-aterima, micolor; truncus al) occipite usque ad caudae basin cingitur anmulis $4 / 4$ angustinsculis schistacen-atris per interstitia aequalia diremtis; in ntroque margine integerrimi, subtus vero abdominali linea schistaceo-atra disseeta sunt; anmuli utplurimm in dorso medio ac versus scutella abdominalia paullo latiores sunt quam ad latera; in spina dorsi adsunt inter lascias, in medio interstitii olivaceo-virescentis, maculac schistace-altac, prima pone occiput conspicua; maculae hae utplurimum rofundae sumt, mox majores mox minores; raro versus truncum infinum in fasciolam ellipticam vergunt; omnium minimae sunt in medio trunci; irides...?

Proportio corporis partium e mensura. Longitudo a rostri apice usque ad caudae finem 17", caudac solius 1" 4\%", capitis 7"'; ambitus capitis immediate pone nares 10", pone oculos 1", occipitis 1", colli supremi 1", tunci in medio 1" 5"', prope anum 1"; cauda ad basin alta est 31/2", in medio et ante apicem $4^{\prime \prime \prime}$.

Habitalioet vitue ratio. Indiam orientalem inhabitat.

OBSERVATIONES. Specimen purissimae conservationis nunc descriptum, communicatum ab amico venerato BoILLEAUD, inspectore summo in rebus navalibus in Portu Gratiae, adest in nea amphibiornm colleclione. Hoc ex India orientali allatum finisse dixit amicus. - Color ruber prope candae basin mox pallescebat; reliquns hucusque restabat; epidermide detrita fasciae schistaceo-nigrae aterrinae funt. SHAwII Hydrus spiralis (Gen. Zool. V.J. T.2. p. 564. t. 125) nisi eadem, attamen species perquam affinis similisque est. De Hydrophe in el anuro sermentibus characteribus potissinum discedere videtur. In Hydro spirali trunci color universalis favus est; truncus vero cingitur annulis intense castaneo-fuscis; inter. quos quidem annulos adsunt maculae rolundae concolores, in trunco medio (?) incipientes, alternantes; canda lata nigra gerit prope basin flavas macnlas; margines ipsius quoque sunt subpellucidi. - Ad ampliorem cero conparationem Angli ipsa verba ponam: Yellowish Hydrus with brown bands, lon. gitudinally confluent bencath. Its length is about two feet, and its habit slender: the body nuch compressed throughout; the back rising into a very sharp carina (in HJdrophe inel anuro non conspicur); the abdomen being also carinated, but having a flattened ed ge of scales sonewhat wider than the rest, and measuring about the fifteenth of an inch in diameter: the head is small, and covered with large scales: the monde wide; the scales on the whole animal moderately small, ovate and slightly carinated: the ground colour is jellow, barred in a leautiful manner from head to tail uith deep chesnust-broun or blackish fascine, each widening on the abdomen, and thus forming a highly distinct and handsome pattern when vieued on each side seeming to constitute so many large, round, yellow spots on a blackish ground: the back, at about the middle, is marked, along its upper part, with a rou of rather large, round, blackish spots situated betucen the fasciae, and so placed as to be in some parts on one side, and in others on the opposite side of the dorsal carina; while some few are seated on thie middle of the ridge itself: this variegation is continued to the tail, which is about an inch and three quarters long, black or deep brown, with a few yellow patches towards its biginning: it is remarkably broad for the size of the animal, and very thin on the edges, so as to be semitransparent on those parts; the carina on the back is so sharp as to surpass in this respect every other species of serpent. - In Hydrophe mel anuro dissectio mihi nullum ostendebat extremitatis posticae vestigium. 



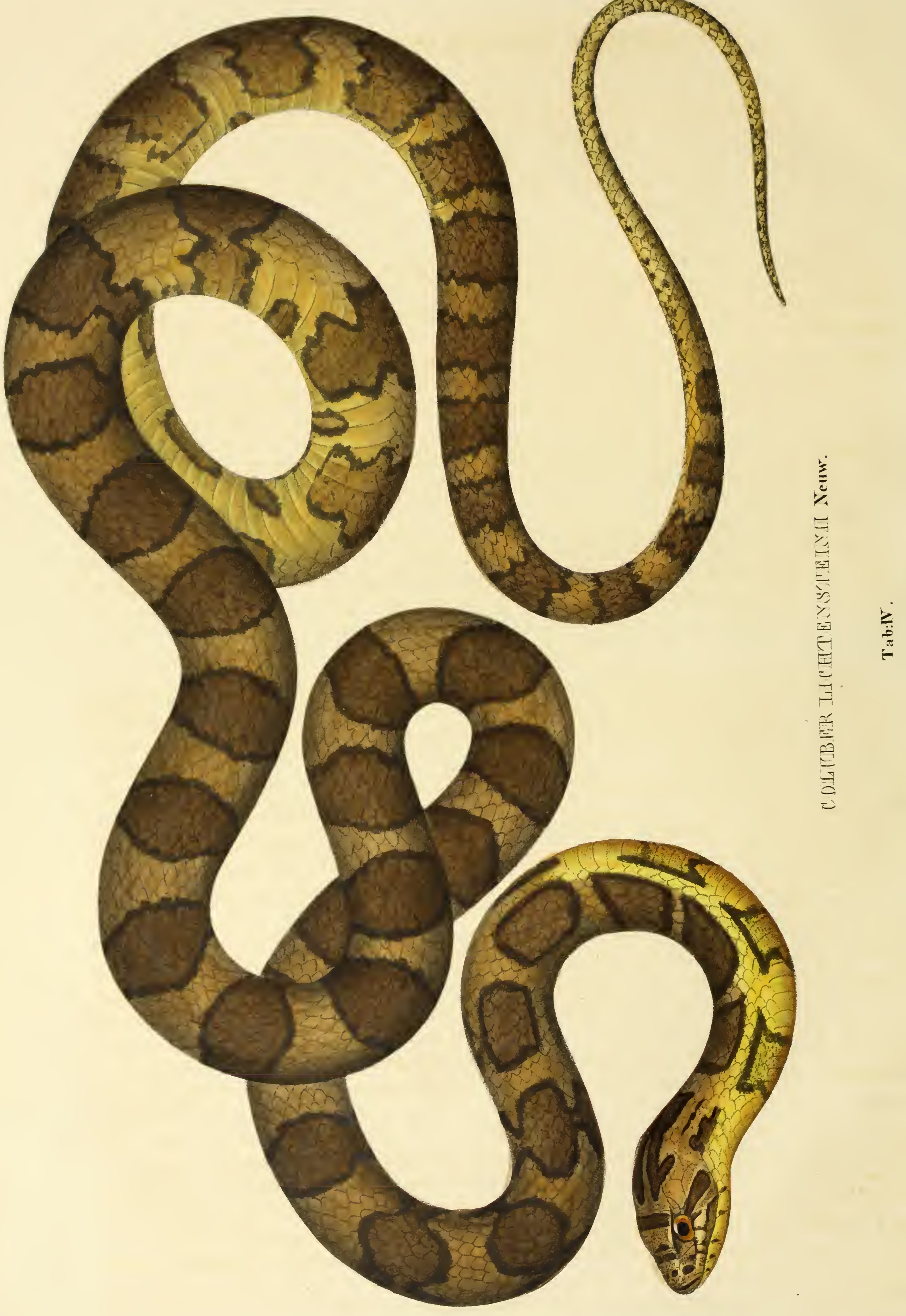




\title{
COLUBER LICHTENSTEINII Neuw.
}

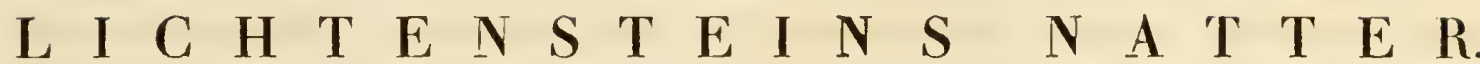

T A B. IV.

Character essentialis. Capite subdistincto aeque alto ac lato, quadrangulo, longiusculo; oculis maximis; trunco subfusiformi tereti-compressiusculo; cauda longiuscula, tereti; squamis laevibus trunci supremi ac caudae subhexagonis, trunci medii rhomboideo-lanceolatis; capitis lateribus laete olivascentibus, macula infra-oculari lata, oblique versus oris angulum ducta nigra, pileo olivascentibrunneo; ejus fasciis tribus nigris et occipitis macula subgeminata longitudinali fusco-nigris; trunco ac cauda supra olivascenti-brunnescentibus, lateribus olivascentibus, illo superius maculis magnis, distinctis, intense brunneis, nigro-marginatis, prinis oblique rhombeis, reliquis orbicularibus, omnibus cum annulis lateralibus scutorum abdominalium, irregularibus, nigris colaterentibus; trunci inferioris parte reliqua pallide flawa; scutis labialibus omnibus flavidis nigro-marginatis; cauda subtus in fundo flavido annulis obsoletis nigricantibus varia. Scut. abdom. 178-183, scut. infracaud. 85-97.

Synonyma: Coluber cap istratus Lichenst. Ver\%. der Dubl. des Berl. zool. Mus. p. 104. Coluber Lichtensteinii Neuw. Beitr. zur Naturg. Brasil. 1. p. 305. (Descriptio optima) Id. Abbild. zur Naturg. Brasil. und Schriften der Kaiserl. Leop. Carol. Akad. T. 12. P. 2. p. 496. t. 46. (F ig. m ediocr.) -? Coluber bifossatus Raddi ment. di Rettili nuor. p. 23.

\section{E S C R I P T I O.}

Corporis forma universalis. Caput subdistinctum, aeque altum ac latum, quadrangulum, subangustum, altum, longiusculum, lateribus perquam perpendicularibus, superius planum, versus rostrum altiusculum, acutiusculo-obtusum parum declive, ante oculos admodum impressum, maxillae marginibus parum prosiliens; facies longa; or is angul us versus occiput ascendens; nares majusculae, perpendiculari-ovales, rostri apici perquam approximatae, in medio scuti nasalis sitae; oculi laterales, maximi, in medio fere capitis siti, vix conspicue versus rostrum deorsum inclinati, pupilla majuscula rotunda; lin g a a nigricans, lateribus albida, pallida apice; scuta: scutum rostrale magnum, altum, medio convexum, versus scuta frontalia anteriora subtriangulari-terminatum, dein lateribus subemarginatunı unde versus basin in utroque latere angulosum; scuta frontalia anteriora mediocria subpentagono-quadrangula, scuta frontalia posteriora anterioribus ultra dimidium majora, subquadrangula, attamen angulo postico externo subrotundato-truncata; scutum verticale campanuliforme, pone trigono-terminatum, mediocre, scutis superciliaribus paullo majus, lateribus subtiliter sinuatum; scuta occipitalia majuscula, irregulariter subquadrangula, pone non acuminata sed rectiusculo-truncata, ibi apice subsinuata, dein lateribus usque ad medium laterale subtiliter oblique truncata ac ibi angulum parvum, prominulum efficientia; scutunı nasale magnum quadrangulum, attamen in utroque latere subrotundato - terminatum; scutum loreum irregulariter quadrangulun, latere interno perquam oblique truncatum, unde parte suprema quam infima multo angustius; scutum oculare anterius unicum subclavatum, magnum, apice superiore sursum flexunı et cum apice scutorum superciliarium apicem anticum attingens, paullo infra haec latiusculum, infra subsubito attenuatum; scuta ocularia posteriora duo parva, angusta, superius inferiore paullo majus apice supremo tri -, infimo subbiangulosum, inferius longius quam altum, margine externo obsolete triangulosum ; scuta superciliaria mediocria, pone rectiusculo - truncata, latiuscula, antice attenuato-acuminati, margine externo supra oculos paullulum prosiliente; scuta temporalia duo oblonga, mediocria, anticum postico paullo majus, illud superius squama interjecta elliptica munitum, ambo in utroque apice triangulosa; scuta marginalia labii superioris 8 , excepta squama ultima subscuti-formi, scuta tria prinı fere magnitudine aequalia, primum parte antica superius suboblique truncatum, secundum suboblique collocatum et tertium quadrangula, quartum et quintum illis vix dimidio majora ejusdem forma, attamen superius versus oculos subintrorsum flexa, sextum magnum, trigonum, attamen apice supremo sub-

Wagles, Aмтн1в. 1 . 
trucatum, margine externo obliqum, septimum omnium latissimum pentagonum, angulum acutum versus oculos vergens, octasum septino dimidio tere mims, quadrangulum, attanen marginis postici angulis obsoletis; scutum labiale parmu trigonum; scnta labialia accessoria mediocria, angusta, pentagona, apicen triangulosum versus scuta mentalia vergens; scuta marginalia labii inferioris 7, duo prima parva, quarlrangula, posticum antico parum majus, tertimn secundo paullo majus ejusdem formae, excepto margine postico infimo subsinıato, quartum omnim maximum, magmın, oblongmm, antice bi-postice subtriangulosum, quintmm quare dimidio bevins, lhombenm, sextum et septimum quinto dimidio fere angustiona, oblongo-subquadrangula, scuta mentalia anteriora mediocria posterioribus breviora, oblonga, antice subtri-, postice in liuea ohliqua bi-angulosit; scuta mentalia posteriora longa, apice postico extrorsum verso divergentia, antice biangulosi, ponc subobsolete trigona; squanae gulares oblongae; den tes parvi, numerosi, aequali longimeline ut in omibus Coluhris formati. 'Truncus elongatus, fusilormis, tereti-compressiusculus, subgracilis, dorso vix compressus, abdomiue rotumlatus, superius per totam suam longitudinem squanarum seriebus 15 olfectus; squamae omues laeves, aequales, exceptis abdomini confinibus majusculis pentagonis, trunci supremi ac caudae subhexagonae, trunci medii rhomboideo-lanceolatae; scuta a bd o m in a lia latiuscula, meeliocria; scutum anale mediocre medio fissun; ca uda longiuscula, teres, a trunco subdistincta, acuta, scutellis inferioribus ommilus medio divisis. Scuta abdominalia 178-183, scuta infracaudalia $85-97$.

Color: Cippitis et tmuci antici latera olivascentia, postici ac caudae in grisescenti-flavidum vergentia, caput et truncus nec non canda supra magis magisque in colorem olivascenti-brunnescentem vergentia, hic et illic atomis uigricautibus ronspersa, vero scuta marginalia labii superioris ac inferioris flavida nigro-marginata, Iaenia transrersa capitis superioris subconspicua in scutis frontalibus anterioribus, altera ante, tertia inter oculos, onues brumen-nigricantes, dnae ultimae subtiliter fulvescenti-limbatae; vitta, verticalibus prorsus concolor, ab oculorum cautho postico versus oris angulum ducta, latiuscula; taeniac duae nuchales longitudinales in utroque scuto oscipitali incipientes, ibi latiusculae et latere externo lineolam obliquam emittentes fasciolis dictis concolores; maculae pone has taeniolas in trunco supreno sitae, interstitio mediocri inter se distantes, magnae, rhomboideae, intense brunneae, taeniola nigra, extus dilute fulvescenti-limbata, cinctae; trunci pars superior reliqua notata est maculis concoloribus et aequalibus interstitiis distantibus, attamen rotundis, versus latera extensis, ibi strictioribus, dilutioribus, margine nigro perquam denticulato et in binorum, rarius trinorum scutorum abdominalium latere externo in annulum irregularem, nigricantem, intus flavidum desinentibus, trunci antici vero maculae primae ab annulis abdominalibus lateralibus striola transrersa nigla, flavido-marginata diremtae, mox iis cum striola transversa flavida approximatae sunt: omnes hae maculae atomis numerosis nigricantibus consperguntur; caudae maculae illis trunci infimi forma et colore similes, strictiores attamen et fere fascii-formes, versus caudae finem minus distincte marginatae; cauda subtus flavida, anmulis indistinctis, ex atomis nigricantibus compositis, subnubilatis obtecta; capitis pars tota inferior flavida, immaculata, interdum scutis nigricanti-marginatis; truncus subtus, exceptis annulis, flavidus, interdun in scutorum medio annulis nigricantibus, obsoletissimis notatus; irides . .?

Proportio corporis partium e mensura. Longitudo a rostri apice usque ad caudae furem 3' 3\%", caudac solius 10\%", capitis 13\%"“; ambitus capitis immediate pone nares 1\%", pone

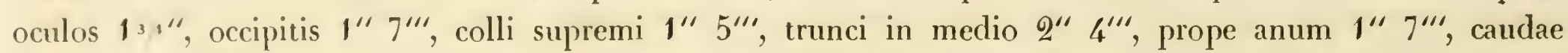
ar basin $1^{\prime \prime} 5^{\prime \prime \prime}$, in medio $9^{\prime \prime \prime}$, in apice extimo $21_{2}^{\prime \prime \prime}$; oculi distant a rostri apice $4 \times 12^{\prime \prime \prime}$.

Habitatio et vitae ratio. Habitat vulgatissimus in Brasilia a Rio de Janeiro usque ad Para locis paludosis, fruticosis, aquam ipsam intrans, parum velox, non timidus, ranis ac bufonibus victitans.

OBSER VATI ONES. Specimina hujus Colubri asservantur in Museo Parisiensi, Lugdunensi Batav., Monacensi ac in collectione mea; in spiritu vini parum pallescunt, abdominis pars solum flavida in sordide albidum vergil. - Animalia juenilia adultis similia sunt, si excipias dorsi maculas in lateribus latiores, sibi approximatiores, intensius coloratas; adulta, quorum nonnulla ultra 7 pedes longa observavi, colore nunquam aut parum variant. - Species Colubro pantherino MERREMII proxima. 



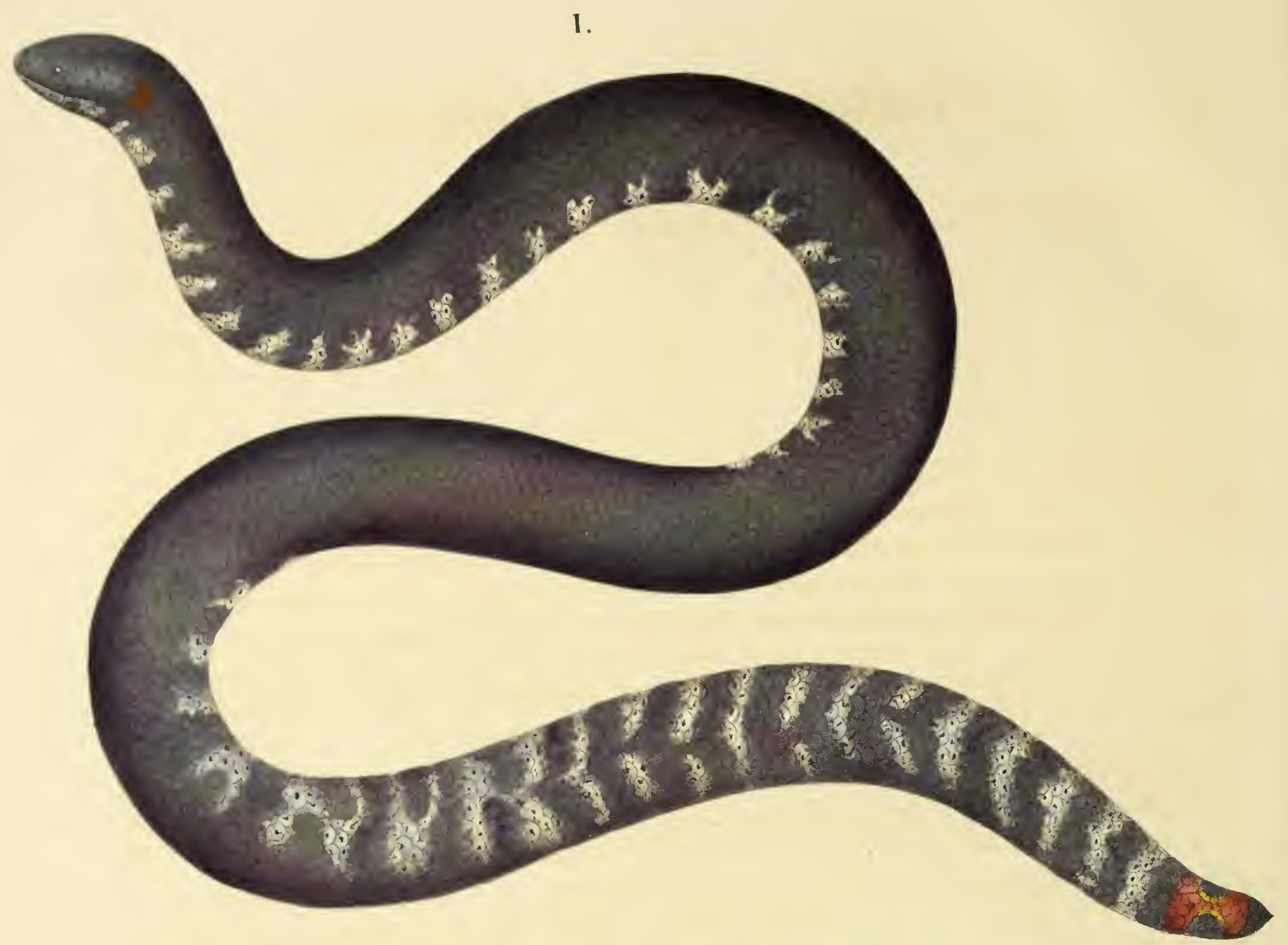

II.

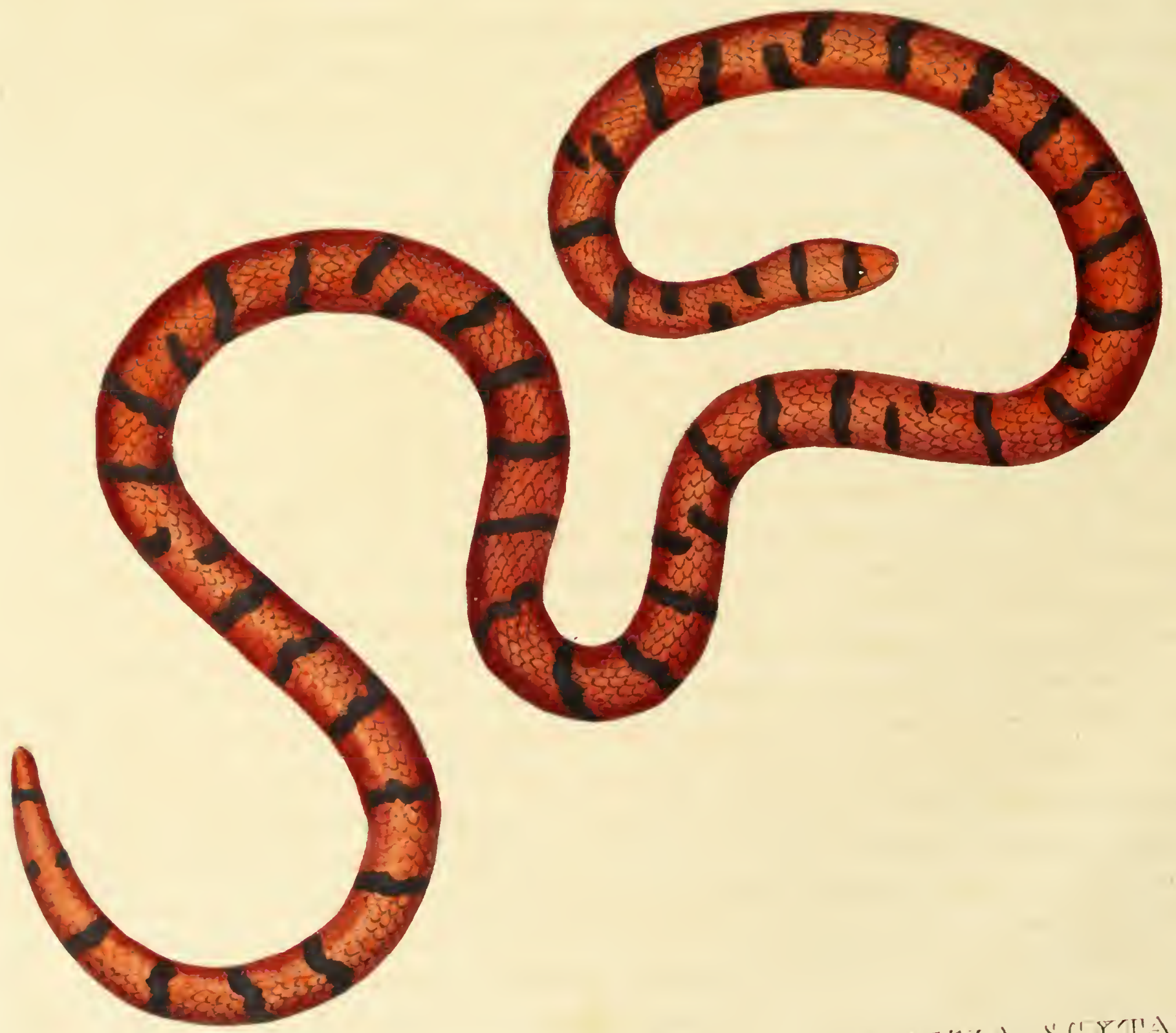

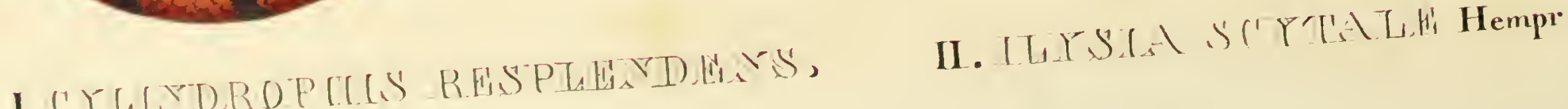
Tab: V. 


\title{
CYLINDROPHIS RESPLENDENS.
}

\author{
S T R A H L E N D W A L Z E N S C H L E I G H E.
}

T A в. V. F. 1.

Character essentialis. Scuto loreo nullo; scuto oculari postico unico, antico nullo; capite plano, declivi, indistincto; trunco crasso, cylindrico, squamis ormibus laevibus, superioribus rhombeis, inferioribus subsemicirculari-angulosis; cauda brevissima, apice conico-ovato; capite et toto trunco supra in fundo nigro laetissime cyaneo-, viridi-ac aureo-viridi resplendentibus, unicoloribus, exceptis fasciolis occipitis duabus (rarius unica) ac (interdum) puncto pone utramque narem aurantio-rubris; trunco inferiore fasciis albis ac cyaneo-nigris frequenter alternantibus vario; cauda subtus macula intense aurantio-rubra notata, cyaneo-nigro terminata. Scutel. infrac audal. 7-9. Adult.

Sinonimis. Scheuchz. Phys. sacr. t. 629. f. F., t. 748. f. 6., t. 660. f. 3. (Iuvenis.) Seba Thes. 2. t. 7. f. 3, 4. (fig. pessimae e speciminibus prorsus detritis) t. 25. f. 1. (figura (coloribus non illustrata) satis accurata, falso a cl. Kuhl pro specie nora habita. Vide Is is 1822. p. 373. Anguis corallina Shaw Gen. Zool. 3. T. 2. p. 282. 1. 131. (icon Sebana) - Ang. squam. abd. 179. et squam. caud. 7. Gron. Mus. ichth. 2. p. 53. Zooph. 1. p. 18. Anguis rufus Laur. Rept. p. 71., Gmel. Syst. Nat. L. p. 1123., Schneid. Hist. Amphib. 2. p. 333., Anguis striatus Gmel. l. c. p. 1119. Eryx rufa Daud. Rept. 7. p. 263. Tortrix rufa Oppel, Merr. Syst. Amphib. p. 84. - Anguis Scytale Russ. Ind. Serp. 2. p. 30. t. 27. (fig. accurata exclusa caudae pictura.) Ilysia rufa Hempr. in litt.

\section{E S C R I P T I O.}

Corporis forma universalis. Animal adultum. Caput indistinctum, a vertice versus rostrum perquam declive, depressissimo-planum, latum, rostro subsemicirculari-obtusatum, lateribus rotundato-declive, occipite subintumidum; oris rictus rectissimus, angulus vix uscendens; nares rostri apici proximae, parvae, punctiformes, antrorsum spectantes, versus angulum externum, anticum scuti frontalis primi sitae; oculi admodum parvi, recti, circulares, scuti superciliaris margine nullo modo prosiliente haud adumbrati, pilei margini proximi, rostri apici parum propiores quam oris angulo; scuta: scutum rostrale mediocre, lateribus rectum, pone trigono-acuminatum; scuta frontalia anteriora subparva, pentagono-quadrangula, posteriora, omnium capitis scutorum maxima, anticis quadriplo et ultra majora; horum angulus posticus externus attingit oculum; fere quadrangula sunt, margine postico subobliqua; scutum verticale, inter oculos situm, aeque altum ac latum, mediocre, fere trigonum, margine antico in medio obsolete angulosum; hoc sequuntur duae squamae majusculae, trigonae; scutum nasale, scutum loreum, scutum oculare anticum non adsunt; scutum oculare posticum unicum pusillum, quadrangulum; scutum superciliare aeque altum ac latum, trigonum, solum cum angulo antico externo oculum attingens; scutum temporale verum nullum, ejus loco squama majuscula trigona, scuta marginalia labii superioris sex, magna, primum omnium minimum, pusillum, immediate infra nares situm, regulariter quadrangulum, ultimum hoc parum majus, pentagonum, secundum anticunı pentagonum, tertium, omnium fere maximum, quadrangulum, margine supremo subobliquo, quartum irregulariter quadrangulum margine supremo postico obliquo, sextum, quinto paullo angustius, pentagonum; scutum labiale medium parvum, trigonum; scuta labialia accessoria majuscula, oblonga; scuta marginalia labii inferioris quinque,

Wagler, Amphib. I. 
secundum omnimm maximum, majusculum, pentigonmu, primum, secundo paullo minns, fuadrangulum, tertimm et quitum oblongo-quadrata, tertimn quarto paullo longius, ultimm pusillum, longiusculnu, margine inferiore subrotundatum; scuta mentalia solum dno, scutiformia; inter amborum margincon internm exstat lovea lincari-longitudinalis; reliquum mentun obtegitur squanis mediocribus, subrhombeis, eae quae proximae scntis marginalibus, reliquis paullo majora sunt; den tes haud mumerosi, validi, acutissimi, palatini maxillanibus non minores; li ng u a teretiuscula, profunde enırginata, apicilus gracillinis. 'Tru cus crassus, longnlus, cylindricus; sq ua ma e onnes glaberrimae, politae, trunci superioris hombeac, inferioris subsenicirculari-angulosae, omnes imbricatae; squamae corporis inferioris eas superioris latiucline paullo superaut, altanen sqquamae seriei abdominis medianae adstantibus forna sintes iisdenque vix majora sunt; ani orificinm mediocre obtegitur squanıis duabus majusculis, in medio interstitio diremtis; $11 \mathrm{~g} \mathrm{~g}$ is extremitatis posticae majusculus latitat in foveola juxta utrumque ani latus; foveolan ipsam obtegit squanı; scutella candac inferiora transversa, hexagona sunt; primum corum, omnimn maximm, antice squanis tribus quadratis limbatum est; cauda brevissima, in apicis conicn-ovati medio mucrone brevissimo, obtusiusculo armata. Scutella infracaudalia T- 9.

Color. Caput supra et ad latera ac truncus totus superior in fundo nigro pro vario ad lucem objectu violaceo-, violaceo-viridi ac aureo-viridi, lapidis Labrador ad instar, egregie resplendent; juxta occipitis latera observatnr lineola ascendens intense aurantio-rubra, alia interdun concolor juxta nucliae latera; interdum adest prope utrancque narem macula punctiformis rubicunda; caput et totus truncus variegantur pagina inferiore fasciis aeque latis albis ac nigro-chalybeis, alternantibus, margine denticulalis; lasciae atbate punctulis nigricantibus conspersae sunt; caudae pars inferior et fascia lateralis intense aurantio-rubrae, fere rubrae sunt, apex extimus chalybeo-ater est; irides obscurae.

I uvenis. Adulto similis, exceptis abdominis fasciis versus dorsum ascendentibus, in dorso medio diremtis; color trunci niger potius in castaneo-fuscum vergit, nitet.

Proportio corporis partium e mensura. Longitudo a rostri apice usque ad caudae finem 2. 1\%", caudae solius 7"“, capitis 11\%"“; ambitus capitis immediate pone nares 13"', pone oculos 11\%", occipitis $1^{\prime \prime} 11^{\prime \prime \prime}$, colli supremi $1^{\prime \prime} 11^{\prime \prime \prime}$, trunci in medio $1^{\prime \prime} 4^{\prime \prime \prime}$, prope anum $1^{\prime \prime}$. 7 ".", caudae ad basin $1 " 6 "$, in medio $1 \% "$, ante apicem extimum 10"; oculi distant a rostri apice 3ะ."

Habitatio et vitae ratio. Habitat satis frequens in Java insula; animal mirae pulchritudinis.

OBSERVATIONES. Specimina Cylindrophios resplendentis exslanl in Museo Parisiensi, Lugdunensi Bat. ac in collectione mea. Auctorum, imo RussELIT, descriptio e speciminibus decoloratis; picturam $e$ specimine vivo debeo cl. de REIN⿴囗十аDT. Color ater, cyaneo-resplendens inclinat ad rufum in exemplaribus per longissimum tempus in spiritu vini asservalis; fasciae albae vergunt in albido-flavidum, nec non caudae macula occipitisque fasciola rubrae albescunl; unde auctorum descriptiones prorsus erroneae. A Cy-lindrophe melanoto (in Museo Lugdunensi Bat. exstante) simili specie ex Java, quam pictam sub nomine Tortricis mel anoti mox expromit amicissimus de REINWARDT differt C. resplendens caudae apice nigro (in C. me la noto albo) fascia occipilali rubra (in C. mel anolo non conspicua) nec non fronte nigra (in Mela noto alba medio macula nigra) fasciüsque trunci inferioris latioribus, sibi approximatioribus - RussEIII Shilay Pamboo (Ind. Serp. 2. p. 32. t. 28) juniorem Cylindrophem resplendentem exhibere videtur; in ista attamen figura maculae duae prope nares, animalijuvenili praecipue propriae, non conspiciuntur. - Bene observavit primus, descripsit ac depinxil MAYER extremilatum posticarum unguem (Verhandl. der Leop. Carol. Gesellsch. der Naturf. B. 4. 1825. S. 831.t.67.f. 5, 7.), tarsum ac metalarsum. 


\title{
IL Y S I A SCY T A LE Hempr.
}

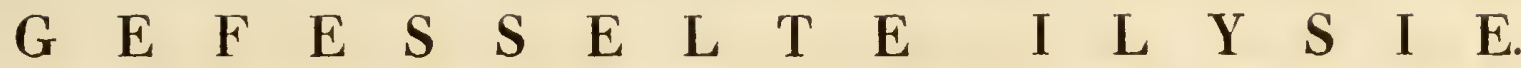

\author{
T А в. V. F. 2.
}

Character essentialıs. Scutts frontalibus posticis omnium capitss maximis, scuto vertıcali quadruplo majoribus; oculis in medio scutelli subrhombei sitis; trunci aequalis, cylindracei, squamis laevibus rhombeis, caudae brevissimae, corpori aequalis, cylindraceae, obtusae hexagonis; capite, trunco ac cauda intense cinnabarino-rubris, fasciis numerosis, margine denticulatis, annuliformibus, attamen supra, ad latera aut subtus alternantibus, aterrimis pictis; annulo capitis prope oculos incipiente, altero ante caudae apicem rubrum nigris, integris. Scutellis hexagonis abdominis $240-258$, caudae $10-13$.

Scheuchz. Phys. sacr. t. 628. f. B., t. 668. f. 2. - SebA Thes. I. t. 84. f. 1. (fig. mal a.), II. t. 30. f. 3. (fig. bona.) Anguis squamis abdominalibus 240, caudalibus 3. Lins. Mus. Princ.-Amoen. Ac. 1. p. 296. Anguis Scytale Linn. Mus. Ad. Fr. 1. p. 21. t. 6. f. 2. Syst. Nat. 1. p. 392. Laur. Rept. p. 70. Gmel. Syst. Nat. L. 1. p. 1121. cum varietatibus $\beta, \gamma$ Latr. Rept. 4. p. 220., Daud. Rept. 7. p. 302. t. 87. f. 1. - Anguis scutis abdomillalibus 227 et squamis caudalibus 14. Grovov. Mus. ichth. 2. p. 54., Zooph. 1. p. 18. Anguis coerulea, A. Íasci a ta Laur. Amphib. p. 70, 71. Le Rouge Bonnat. Ophiol. t. 42. f. 7. Lacíp. 2. p. 450. t. 19. f. 7. Anguis rubra Latr. Rept. 4. p. 224. Tortrix Scytale Oppel., Cuvier, Merr. Syst. Amph. p. 83. Ilysia Scytale Hempr.

\section{E S C R I P T I O.}

Corporis forma universalis. Caput indistinctum, trunco aequale, depressum, superius lateribus devexum, apice obtuso rotundato-truncatum, mandibula quam maxilla paullo brevior; oris rictus rectus, angulo recto; nares in rostri apice fere extimo in scuti frontalis antici margine extremo sitae, punctiformes, subparvae, introrsum spectantes, intus plica pusilla, subtereti, cutanea munitae; oculi fere in medio scutelli subrhombei siti, nullibi protuberantes sed cum relicua capitis planitie aequales, laterali-superni, minutissimi, circulares, pupilla minima rotunda; scuta: scutum rostrale mediocre pone trigono-terminatum, margine infimo parum emarginatum; scuta frontalia anteriora parva fere pentagona, margine postico recto; scuta frontalia posteriora ingentia, omnium scutorum capitis maxima, latere externo angulum ante medium emittentia obtusiusculum, pone trigono-terminata; scutum verticale parvum, pone attenuatum, subconico-terminatum, antice trigonum, scutis frontalibus posticis quadruplo minus; scuta superciliaria nulla; juxta utrumque scuti verticalis latus adest squama majuscula, subrhombea, quam lateraliter sequitur secunda, latior, obliquo-hexagona, scutum temporale quodammodo repraesentans; reliqua capitis pars superior obtegitur squamis, quarum tres scutum verticale sequentes, reliquis occipitis parum longiores attamen earumdem fere forma sunt; scuta lorea et ocularia, tam anteriora, quam posteriora non adsunt, scuta marginalia labii superioris sex, quorum ultimum squamiforme, tertium omnium maximum permagnum, supra trigonum, primum mediocre regulariter quadrangulum, secundum hoc paullo majus quadrangulum, quartum primo paullo majus quadrangulum, quintum quarti magnitudine attamen superius angulum parvum emittens; scuta marginalia labii inferioris sex, quorum ultimum squamiforme; tria antica majuscula aequali fere magnitudine, quadrangula, quartum quadrangulum primo vix dimidio minus, quintum quarto paullo minus postice subovatum; scutum labiale medium magnum, acuminato-trigonum; scuta labialia accessoria angusta, medio longiora, pone trigono-terminata; scutorum mentalium par unicum; maxima sunt haec 
scuta, lattuscula, pone ovato-trigona, antice latere externo paullo biangulosa; medio includunt squamulam oblongan, pone trigonam, antice stricte acuminatam; pone utrumque scutum adsunt praeterea squamae duae rhombeae, reliquis, quibus catera capitis inferioris pars obtegitur, paullo majores; harum antica tertia fere parte major quam postica; gulae squamae hexagonae, menti reliquae thombeate. Truncus cylindraceus, elongatus, gracilior, squamarum seriebus 19; squanae trunci rhombeae, laevissimae, caudae fere hexigonae; scutella abdominis ac caudae transversa, angusta, hexagona, caudae integra; scutellum :mi oriticium obtegens semilunulare integrum; nnguis extremitatis utrinque juxta ani latera minutus in fiveoli, superius squama tecta, latitans; cauda brevissima corpori aequalis, cylindracea, apice parum attenuato obtusissima. Scutella abdominalia $240-258$, scutella infracaudalia $10-13$.

Color: Caput, truncus et cauda intense cinnabarino-rubra; annulus aterrimus, latiusculus, regularis cingit caput, incipit prope oculos et ambit oris angulum; annulus similis, integerrimus cingit caudam aute apicem, qui ruber est; fascia caudae altera concolor, latior, utphurimum latissima omnium corporis, adest supra suan hasin, sed desinit juxta ani orificii latus; truncus totus obtegitur fasciis aterrimis munerosis, quae aequalibus interstitiis inter se distant; earum latitudo mediocris, margo undulatodenticulatus; mox alternant in dorso medio, mox in lateribus, saepissime in dorso; squamae rubrae dorsi medii variegantur apice maculis pusillis, interdum subobsoletis, nigricantibus, in animale juvenili conspicuioribus quan in adulto; lingua pallida est; irides..?

proportio corporis partium e mensura. Longitudo a rostri apice usque ad caudae finem $2{ }^{\prime} 7 \% "$, caudae solius 10\%", capitis 10"“; ambitus trunci pone occiput et in medio 13\%", prope anum $I^{\prime \prime} \ell^{\prime \prime \prime}$, caudae ad basin ac in medio $1^{\prime \prime} 1^{\prime \prime \prime}$, in apice extimo $7^{\prime \prime \prime}$; oculi distant a rostri apice $3 \times 2 \cdots$

Habitatio et vitae ratio. Habitat in Surinano, in Brasiliae provinciis Amazono flumini confinibus ac, ut dicitur, in Guiana et Cajenna; vivos edit foctus; victitat parvis amphibiis. ${ }^{*}$ )

OBSERVATIONES. Specimina Scytales, in Surinamo vulgatissimae, conspiciuntur in plurimis museis :oologicis. Color ruber pulcherrimus post animalis mortem mox in album vergit annulique nigri plus minusve ad fuscum sive plumbeum colorem inclinant; unde auctorum varietates Scytales. Sola BonNA-

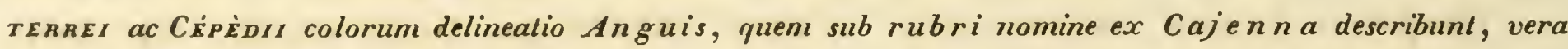
est. - Picturam Sebanam utramque (Il. t. 75. f. 2, 3.) pessimam, scula ventralia et subcaudalia monstrantem huc ponendain esse vix dubito; ïs innituntur LAVRENTII, GMELINI ac SCHNEIDERI Anguis corallinus et Anguis ater. - Extremilatis posticae unguis a cl. MAYER primo detectus, descriptus et delineatus est in libro titulum gerente: Verhandlungen der Leopold. Carol. Akad. der Naturforsch. Bd. 4. S. 829. 1.67.f.6. Unguis facile reperitur; in foveola angusta, juxla ani latera sila, superius squama tecta, latitat. Unguem hunc in speciminibus tredecim a me observatis nunquam quidem extra diclam foveolam inveni, nihilominus, ut in felibus, emissilis esse videtur.

-) In specimine a me dissecto parun ultra pedem longo inveni Caeciliam lumbricoïdem pedalem, in solo capite laesam. 



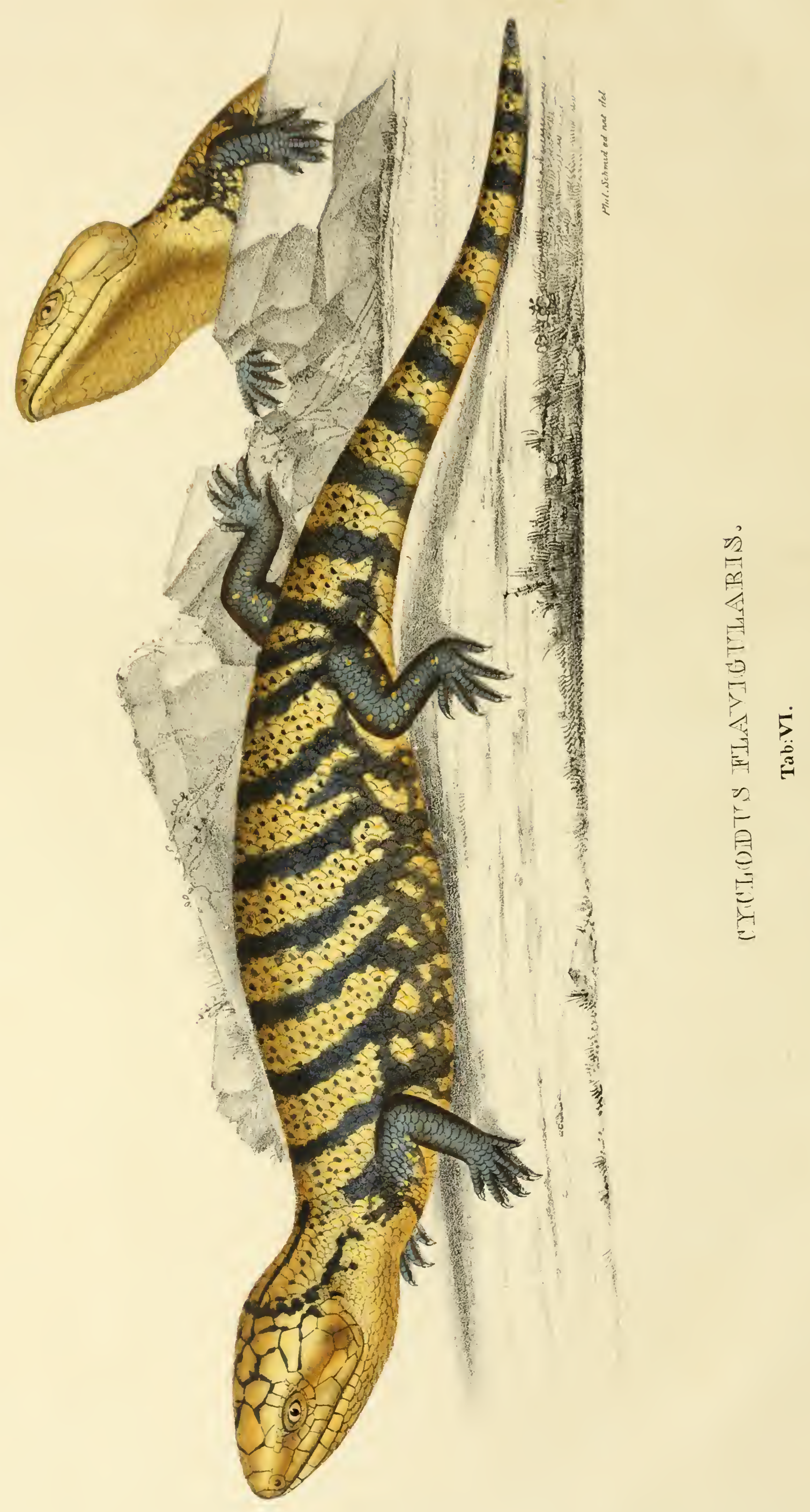




\title{
CYCLODUS FLAVIGULARIS.
}

\author{
G E L B K E H L I G E R K R E I S Z Ä H N L E R.
}

\section{T A в. VI.}

Character essentialis. Capite maximo tetragono-pyramidali scutis verticalibus quatuor, quorum antico, omnium maximo, et postico pyramidalibus, duobus mediis anguloso-rotundatis; squamis laevibus hexagono-semicircularibus; cauda compressa culmine obsolete quadricarinata; uropygio plano; capite toto ochraceo-aurescente, solum scutis verticis ac occipitis nigricanti-marginatis; trunco et cauda subsordide ochraceo-aurescentibus fasciis 19 fusco-aterrimis, in abdomine variolosis, in caudae pagina infima regulariter confluentibus; fascia occipitali subarcuata, angusta, nigra, e medio et ex utroque latere lineam longitudinalem emittente; pedibus fusco-nigris, subtus, raro supra, maculis pusillis flavis pictis. Scutel. infracaud. 53.

SYNONYMIA: 0

\section{E S C R I P T I O.}

Corporis forma universalis. Caput maximum tetragono-pyramidale, temporibus perquam intumidum et protuberans, dein lateraliter usque ad rostri apicem applanatum, quare verticis margine angulosum, ante oculos vix impressum; tota capitis pagina superior applanata, obsolete supra frontem conspicuius convexa, apex extimus obtuso-acuminatus, maxillae ac mandibulae margines verticales; mandibula maxillae longitudine; nares rostri apici proximae, laterales, mediocres, subelliptico-rotundatae, antrorsum spectantes, in foveola sitae; oculi mediocres, aeque distantes ab auribus ac a naribus, verticis-margini confines, a margine maxillae longe distantes; scuta: scutum rostrale mediocre parum altum triangulum, in utroque apice laterali scutello minimo terminatum; scutum superius sequens majusculum hexagonum, apice antico angustiore; hoc sequuntur scuta duo, unum alteri lateraliter-adnatum aequalis magnitudinis ac formae, majuscula, pentagona, juxta scutorum verticalium primum perquam oblique truncata; scuta verticalia quatuor, quorum "primum omnium capitis longissimum, elongato-campaniforme, antice trigonum, parte posteriore angustato-lanceolatum, scuta verticis sequentia duo lateraliter sibi adnata aequalis magnitudinis et formae, mediocria, subanguloso-rotundata, scutum verticale posticum latiusculum, primo tertia parte brevius, triangulo-hexagonum, lineolis duabus longitudinalibus exaratis, parallelis (an in omnibus speciminibus?); in utroque hujus scuti latere extat scutum majusculum fere pentagonum, quod pone sequitur aliud paullo minus subobliquum, fere quadratum, subsequentibus scutellis ac scutis, irregulari-quadratis; scutellum nasale parvum situm est in scuti majoris parte antica inferiore; hoc scutum majus pone subrotundatum, versus scutum rostrale angustato-acuminatum, minus, nares recipiens, potius pro plica exarata majoris habendum est; hoc scutum inter et oculum intermedia scuta duo, quorum anticum oblongo-subquadratum, magnum, posticum pentagono-quadratum, antico minus dimidio; oculus cingitur supra et infra scutellis quatuordecim, magnitudine inter se paullo discrepantibus, subquadrangulis; scutella superciliaria adsunt quinque, quorum primum mediocre triangulari-pentagonum, secundum maximum pentagonum, versus scutum verticale primum praecipue dilatatum, tertium angustissimum, secundo minus altum, quartum subpentagonum, tertio paullo brevius attamen multo latius, ultimum minutissimum, fere triangulare; scuta temporalia extant in utroque latere duo (an tria?) magna, quorum inferius maximum, oblongosubquadratum, superius hoc dimidio fere minus altum, anguloso-cuneatum, parte strictiore antrorsum spectantes; haec scuta duo, sicut scuta labialia 7 et 8 , foveolis numerosis obsoletissimis obsita sunt; scuta terminantur scutellis polygonis usque ad aures; scuta marginalia labii superioris $10 ; 2,3,4,5$, 6 aequali altitudine sed inaequali latitudine, quadrangula, primum quadrangulum infra nares situm dictis parum minus altum, septimum iisdem paullo altius, subpentagonum, octavum omnium altissimum ac latissimum, permagnum, pentagonum, nonum oblongo-quadratum antecedente multo minus altum, ultimum minutum quadrangulum; scuta marginalia labii inferioris 11 , omnia aequali fere altitudine ac latitudine, exceptis secundo et tertio reliquis paullo latiora ac altiora, omnia subobliqua et quadrangula; scutum labiale medium latiusculum margine postico rectiusculo-truncatum; pone id observatur scutum 
transversum margine postico in medio angulum subobsoletum emittente, dein sequuntur infra utramque seriem scutorum labialium scuta quaterna, labialibus inulto nıjora, inagna, irregulariter quadrangula, laevissima; duo ultina lıorum scutorum a labialibus interjectis squamis diremtae sunt; reliqua capitis pars inferior obtegitur squamis cuti adpressissimis, lacvissimis, transversis, hexagono-semicircularibus, quae vero inter scuta infra-labialia extant potius polygonae sunt; n ares mediocres, verticali-ellipticae, latiusculae, margine antico squamulis tribus semicircularibus munitae, postico squamulis pusillis ejusdem formae, numerosis obsitac; dentes palatini nulli, maxillares postici corona orbiculares, antici ac primores parvi, angusti apice obtusi; lingua carnosa non emissilis, plana, lanceolata, pone perquam dilatata, apice integra sed margine ibi sursum subplicata, pagina lata superiore numerosissime et subtilissime papillosa; tr u n cus tereti-compressiusculus, longulus, medio paullo incrassatus, dorso medio obtusissime compressus, supra liumeros et uropygium applanatus; collum mediocre, lateribus compressum, supra dilatato-applanatum; squama c colli ac totius trunci laeves, hexagono-semicirculares, imbricatae, in collo supremo quanı in reliquo trunco, praesertim pone occiput, latiores ac per series longitudinales regulares dispositae, in trunco undique aequali magnitudine; in uropygio applanato, et subconspicue in spina dorsi, squımae medio longitudinaliter compressae sunt, quo fiut carinae obtusae ac lineae in uropygio ac caudae culmine (ubi aequo nıodo conformatae apparent) longitudinales; pedes quatuor digitis aequali longitudine; pedes breviusculi, robusti, brachium ac femur compressa, antibrachium depressum, tibia teres; squamis obteguntur transversis fere hexagonis; digitorum teretum, robustorum, brevium squamae superiores transversae, quadratae, omnes aequali forma et magnitudine, excepta squama rhizonychium obtegente reliquis paullo majore perquam gibba, squamae inferiores superiorum forma in eo differunt, quod margo anticus prominulus sit, quare rugosae apparent; palmae ac plantae obteguntur squamulis gibbis, verruciformibus attamen lacvibus; u n gues parvi, parum arcuati, acutissimi, pagina inferiore longitudinaliter excavati, in ommibus digitis aequali longitudine; an us transversus antice marginatus est scutellis 6 majusculis, margine postico subrotundatis, laevibus; cauda longiuscula, compressa, inferius rotundata ac scutellis hexagonis 53 (in specimine meo) transversis, lateraliter squamis hexagonis, superius squamis supra descriptis obtegitur; apex extimus conico-acuminatus.

Color. Caput totum ac collum infimum ochraceo-aurescentia, immaculata, exceptis pilei scutis, praesertim verticis, fuscescenti-lavatis subtiliter nigricanti-limbatis; pedes digitique fusco-nigri, paullo cyaneo-micantes, subtus, raro supra, maculis pusillis, flavis picti; palmae ac plantac olivascenti-fuscescentes; ungues corneo-albidi; truncus et cauda ex subsordido-ochraceo aurescentes fasciis 19 aequalibus interstitiis diremtis, angustiusculis, fusco-aterrimis, paullo cyaneo-micantibus ornata; fasciae hae caudam in morem annulorum prorsus amplectuntur ac latiores sunt quam in trunco, ubi ad latera stricta, in dorso medio paullo latiores fiunt, in abdomine rero marmoreo-dissitae maculis flavis interpositis lacte variegantur; occiput terminat linea subarcuata fusco-nigra (arcu antrorsum spectante) quae e medio lineanı concolorem emittit rectam supra nuchae medium extensam, et in utroque latere in taeniam concoloren, versus humeros porrectam desinit; prope brachii articulationem taeniolae duae, mox divergentes, antrorsum spectantes nascuntur fusco-nigrae; brachium versus articulationem flavo-variegatum; lingua ac palatum incarnata; irides..?

Proportio corporis partium e mensura. Longitudo a rostri apice usque ad caudae finem

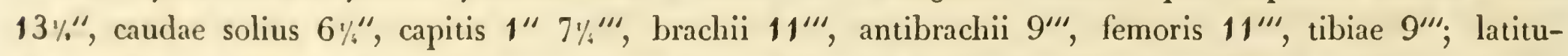
do capitis immediate ante oculos $6 " \prime$, supra nares $2 \%{ }^{\prime \prime \prime}$, ejusdem altitudo ante illos fere $7{ }^{\prime \prime \prime}$, ante has $3 \%$ "'; oculi distant a rostri apice fere $8^{\prime \prime \prime}$.

Habitatio et vitae ratio. Habitat....?

OBSERVATIONES. Specimen descriptum, nitidissimum exstat in collectione mea. Spectat forsan aucto. rum Scincus Gigas hoc animal. Quis vero e verbis ,Scincus giganteus, albus, fasciis 19 nigris" speciem certo colligere valeat? MERREMII S cincus Gigas (Tentam. Syst. Amph. p. 75. n. 17.) differt tolo coelo; adest hic ab eo descriptus Cjclodus in Museo Lugdunensi Bat., fusca taenia pone oculos insignis, pedes 2 longus. Scincus nigro-luteus e Nova-Hollandia, qui describitur ac depingitur a cl. Quor et G.IMARD (Voy. du Capit. FREYCINET, Part. Zoolog. T. 41.) spectat hoc genus ac species affinis est, quae a Cyclodo flavigulari colorum distributione, capitis praesertim, maxime variat. Adhuc huic generi inserendae sunt SHAwI Lacerta occidua ex Antillis insulis ac Lacerta scincö̈des e Nova Hollandia. (WhITE Journ. p. 242. c. Tab.) 


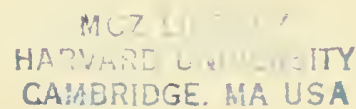





\title{
HELICOPS CARINICAUDUS.
}

\author{
K I E L S C H W E I F I G E S S C H E E L A U G E.
}

T A B. VII.

Capite depresso vix distincto; trunco tereti, aequali, longo, squamis rhombeo-hexagonis, dorsi supremi antici obsolete-, inferioris magis magisque conspicue-, infimi ac caudae tenuis totius perquam carinatis; capite, trunco toto ac cauda latere superiore fuliginoso-nigris, subtiliter olivaceolavatis, splendentibus, unicoloribus, squamarum serie ultima maculis parvis nigricantibus varia; taenia utrinque ad trunci superioris latera inferiora flava a capite usque ad anum ducta flava; capite, trunco et cauda subtus flavidis, triplici serie macularum guttiformium nigrarum, in trunco supremo ac in cauda solum duplici. Scut. abd. 137-148, scut. infracaud. 50-55.

Symonyia: Coluber carinicaudus, Prinz von Neuwied. Beyträge zur Naturgesch. Brasil. Bd. 1. S. 300 . (Descript. opt.) Id. Abbild. zur Naturgesch. Brasil.

\section{E S G R I P T I O.}

Corporis forma universalis. Caput vix distinctum, obtusum, depressum, latum, lateribus maxillae prominulis convexiusculum, rictu reverso-subarcuato, latiusculo; facies admodum brevis, ante oculos impressiuscula, latiuscula; nares subsuperae, minutae, punctiformes; oculi subparvi, oblique collocati, large inter se distantes, rostri apici subapproximati, laterali-subsuperi, longiusculorotundati; scuta: scutum rostrale latiusculum, pentagonum, basi quam apice paullo angustius, angulo supremo medio acutiusculum; scutum frontale anticum unicum subtrigonum, versus scutum rostrale perquam attenuatum, pone admodum dilatatum et margine rotundatum; scuta frontalia posteriora duo mediocria, subirregulariter quadrangula attamen versus scutum verticale angulo brevi, subprominulo, extus versus scuta lorea deflexa; scutum verticale longiusculum, antice et postice fere aeque latum, pentagonum; scuta occipitalia' magna, versus scutum frontale suboblique truncata et medio angulo parvo prominulo, postice acuto-angulosa, extus ibi oblique truncata; scutum nasale quadrangulum, majusculum a naribus superne sitis usque ad marginem infimum fissum; scutum loreum mediocre, quadrangulum, scutum oculare anterius unicum strictissimum, altiusculum, medio impressum, subtus et supra biangulosum; scutorum ocularium posteriorum superius mediocre, subirregulariter quadrangulum, inferius paullo majus quadrangulum, versus oculum oblique truncatum; scuta superciliaria mediocria, versus rostrum subtrigono-acuminata, postice biangulosa, supra oculos non procumbentia sed ibi subsinuata; scuta temporalia, bina utrinque, majuscula, superius inferiore majus, versus scuta ocularia irregulariter tri-, pone bi-angulosum, inferius ibidem tri-, antice in linea obliqua bi-angulosum; squamae occipitales majusculae irregulariter hexagonae, laeves; scuta marginalia labii superioris 8 , marginibus perquam distincta, medio admodum convexo-protuberantia, omnia, excepto ultimo postice subobtuse acuminato, quadrangula, duo prima omnium minima, tertium his parum majus, sextum et septimum omnium maxima aequali magnitudine, magna, margine inferiore versus orem inflexa; scutum labiale medium minimum trigonum; scuta labialia accessoria angustiuscula versus scuta mentalia trigono-lanceolata; scuta marginalia labii inferioris 9, 
duo prima minuta aecuali magnitudine, quadrangula, tertim his dimidia parte majus, subquadrangulum, quintum quarto ertia fere parte majus, magine postico in medio angulo prominulo, sextum, septimmm, octarmm et nomm nuagnitudine magis magisque decrescentia, imegrulariter quadrangula; scuta

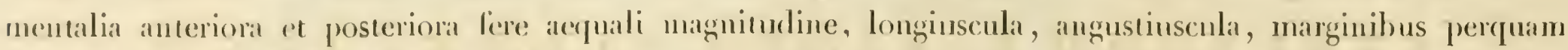
distincta, anteriora apice antico subtrigona, postico oblique truncata, subliangulosa, posteriora pone suhulato-acuminata; squamae gulares oblongae, angustac 6; dentes ut in ommibus speciebus hujus generis formati; lingna stricti, teres, bifirca, longe emissilis. Truncus longus a capite usque ad anum aequali crassitie, teres, abdomine rotundatus, squamam seriebus medio 19, versus anum seriebus 17; sy 11 a 11 a e hexangulo - rhomboidales, dorsi totins medii cárina obsoleta, rersus truncum posticum magis magisque conspicna ac magis magisque squanas laterales quoque occupante, in omnibus squanis a medio trunci usque ad anum conspicua et perquan distincta, munitae, exceptis seriebus squamarum scutis abdominalibus confinium duabus lacvibus; scuta abdominalia mediocria, angustiuscula; scutum anale medio lissun1; cauda subulato-acuminata, tenuis, teres, aculeo parro, subcorneo, recto terminata, squamarum seriebus 8; squanae dorsalibus forma similes, solum breviores, sine exceptione peryuan carinatae; scula infracaudalia ommia divisa. Scula abdominalia 137-148, infracaudalia 50-55. CoLor: Capput, truncus et cauda supra linliginoso-nigra, subtiliter olivaceo-lavata, immaculata, attanen pro vario ad lucem objectu egregie lazulino-resplendentia ac squamae ultinae seriei maculis obsoletis parvis, niggris notatae; labia naxillac, taenia lata, rectissima utrinque juxta trunci superioris latera inferiora a capite usque ad anm ducta atc totum caput subtus flava; scuta abdominalia onnia quan taenia lateralis pallidius flava, horum unumquodque guttis tribus aterimis, pro vario ad lucem objectu egregie lazulino-resplendentibus, scnti marginem anticum non altingentibus, notata, unde in abdomine series tres longitudinales guttularum; guttulac intennediae circiter post scutum vigesimum, primum obsoletae, sensim conspicuiores incipiunt et multun ante scutum anale evanescunt; scutum candae unumquodque flavum guttula subnubilata nigra notatum, unde in hac guttularum series duae longitudinales, rectae; guttulae in ultimis scutis hic et illic subnigricanti-conspersis evanidae; squamae scutis confines macula intermedia obscuriore nigra notatae; lingua nigra basi pallida; irides flaro-fuscae.

Proporlio corporis partiume mensura. Longitudo a rostri apice usque ad caudae finem

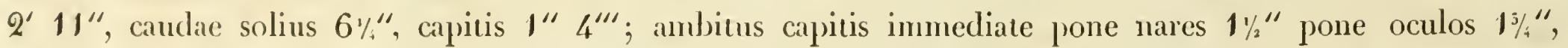
occipitis 2" 2"', colli supremi 2", trunci in medio 2\%", prope anum 2" 1 ", caudae ad basin 18", in medio 10"', in apice extimo 4\%"'”; oculi anoti sunt a rostri apice vix $3^{\prime \prime \prime}$.

Habitatio. Habitat in Brasiliae provincia Bahia, ac in sylvis magnis prope flumen Itapémirim, ubi lecta est a Principe Neoredano.

Fitae ratio: ..?

OBSERVATIONES. Specimen delineatum, masculinum, purissimae conservationis, a nauta ex Brasiliae provincia Bahia allatum, emi in Portu-Gratiae; adest in mea animalium collectione. Seren. Principts Vedani Neovedani descriptionis pars maxima egregie in illud quadrat, attamen dorsi medii supremi squamas obsolete carinatas describere omisit hic historiae naturalis fautor excelsissimus. - Colores Helicopos carinicaudi in spiritu vini asservati partum pallescunt; corporis solum partes flavidae ac favae in coloren albidum, sordide rubicundum quast vergunt. - Coluber plicatilis ac Coluber ergthrog rammus species affines sunt et hoc genus spectant. 
HARVARD U: WE SITY

CAMERIDGE. MA USA 


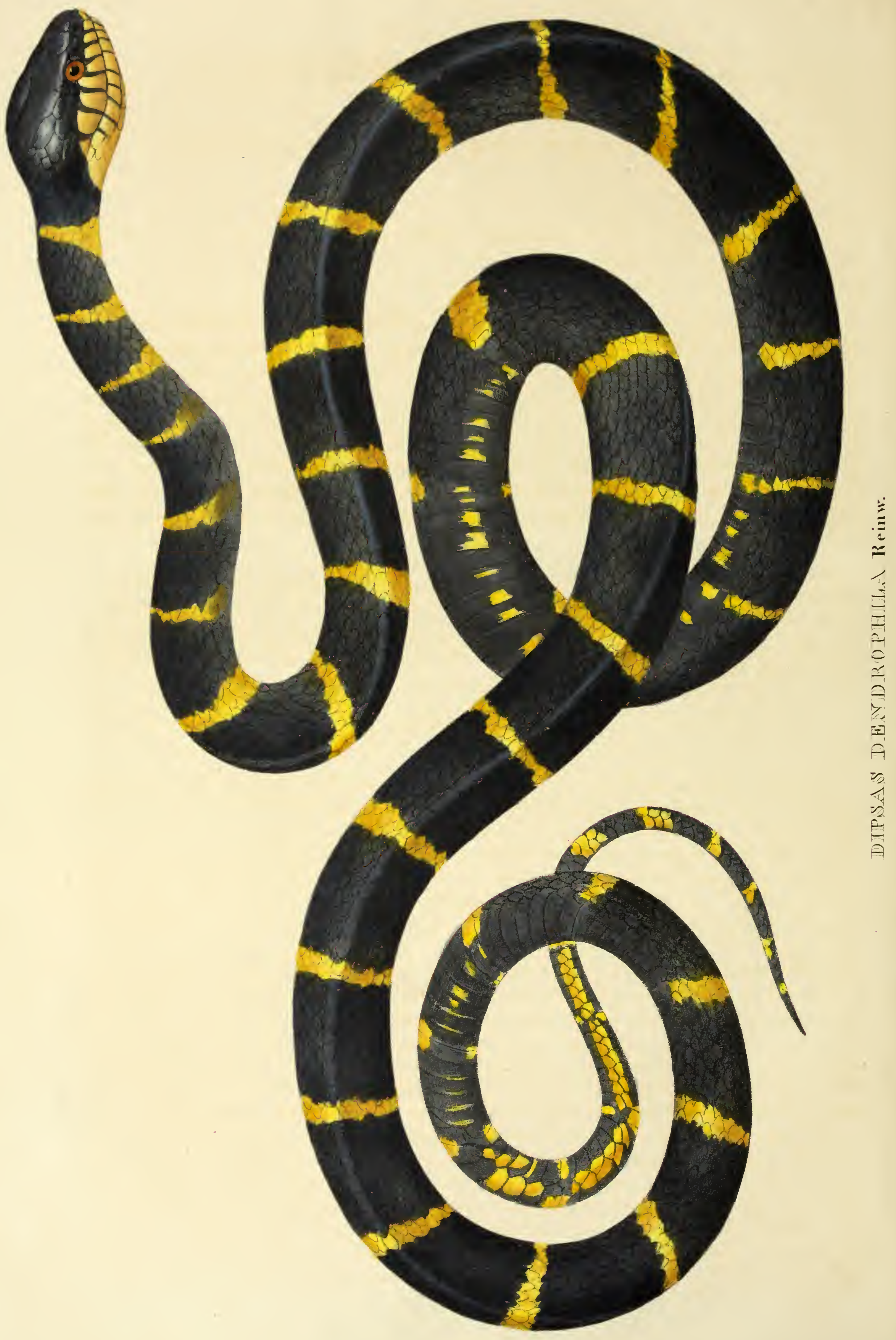




\title{
DIPSAS DENDROPHILA Reinw.
}

\author{
B A U M L I E B E N D E K O P F
}

\section{T A в. VIII.}

Character essentialis. Trunco fusiformi percompresso, dorso cestriformi-compresso; squamis lanceolato-imbricatis laevibus, spinae dorsi majoribus, regulariter hexagonis, candae omnibus hexagonis; scutis frontallbus perquam convexis; capite supra, trunco ac canda aterrino-cyaneis; scutis labialibus, maxillae ac mandibulae, flavis margine apicali atro; capitis parte infera gillaque aureo-flavis, immaculatis; trunci latere fasciis aureo-flavis 36-40 angustis aureo-flavis, in spina dorsi diremtis, in trunco postico subconfluentibus, juxta scutorum abdominalium latus externum latioribus ibidemque desinentibus; cauda aureo-flavo annulata, annulis subtus latioribus, interdum inter se linea longitudinali concolore connexis; reliqua trunci inferioris parte nigra, subolivaceo-lavata, antica solum maculis aureo-flavis variegata. Scut. abd. 223-225, scut. infracaud. $106-107$.

Synonyia: Scheuchzer Physico-Sacr. t. 662. f. 11. (fig. mediocr.) Seba Thes. 2. t. 21. f. 1. An tabula Scheuchzeriana? Figurae ambae a cl. Merrem inter synonyma sui Colubri Caninana falso receptae.) Coluber peruvianus Shaw Gen. Zool. V. 3. T. 2. p. 483. (Descriptio e figura Sebana.) Dipsas dendrophila Reinw. in Mus. Lugd., F. Bo ie Isis Bd. 20. S. 549.

\section{E S C R I P T I O.}

Corporis forma universalis. Caput distinctissimum, breve, perquam ovato-incrassatum, occipitis lateribus admodum intumidum, superius inter oculos impressiusculum, laterum angulis obsoletis subrotundatum, ante oculos compressiusculum, supra nares perquam convexum, apice subtrumcato-obtusum; maxillae labia, praesertim versus oris angulum, convexo-prominula; maxilla quam mandibula paullo longior; oris angulus adscendens; nares rostri apici approximatae, laterales, majusculae, rotundae, introrsum spectantes; oculi majusculi, circulares, prominuli, laterales, rostro quam oris angulo multo propiores, supremo scuti superciliaris margine externo non adumbrati; scuta: scutum rostrale mediocre subsemicirculare, margine externo obsoletissime angulosum, margine infimo vix sinuatun sed ante eum paullo impressum; scuta frontalia anteriora duo mediocria, quadrangula, latere externo oblique truncata, perquam convexa; scuta frontalia posteriora anticis duplo fere najora, admodum convexa, subpentagona, latere externo usque ad scutum loreum deflexa; scutum verticale mediocre, latiusculunı, pentagonum, antice recto-, postice trigono-terminatum; scuta occipitalia majuscula, antice rectiusculo-, postice trigonoterminata, postice latere externo perquam oblique truncata; scutum nasale anticum mediocre, subquadrangulum, pone lunulari-emarginatum, scutum nasale posticum antico vix majus, concavo-lunulare; scutum loreum mediocre subtriangulum; scutum oculare anticum unicum magnun, subquadrangulum, multo altius quam latum, apice supremo scuti superciliaris marginem anticum attingens, scuta ocularia posteriora duo, mediocria, inferum superiore paullo minus oblongum, subovato-angulosum, superum pentagonum, fere aeque latum ac altum; scuta superciliaria scuto verticali paullo breviora eodemque paullo minus lata antice trigono-, postice recto-terminata; scuta temporalia utrinque duo, subsquamiformia, inferius admodum elongatum, angustum, in utroque apice trigonum, superius brevius polygono-oblongum; sequintur nunc squamae plures majusculae, polygonae, quarum maxima pentagono-hexagona immediate pone scuta occipitalia; scuta marginalia labii superioris 8 quorum penultima maxima, antica mediocria; 
ah antico nsque al pentumm magnitudiue magis magisque crescunt; primum scutum triangulum, secmudum quadrangulum, tertimu subpentigonum, quartum quadrangulum, quintum et sextun altitndine aequalia fere quadrangula, quintum tantum apice supreno juxta oculnu paullo truncatum, septimum omıimm litissimm, attamen dnorum pracedentium altitudine, adnodum latum, pentagonum, octavum septino dimidio lere mims quadranguhum, panllo longius quam ahum; scntum labiale parvum, latinsculum, subtrigonum; scuta labialia accessoria pone elongato-lanceolata, mediocria; scuta marginalia 10, sextum omminm maximmm, magum, primmn parvum subquadrangulum, secundum, tertium, quartum "t quinmm aequali fere altitudine et latitudine, subquadrangula, solum versus scuta mentalia subacuminata, sextmm magmm, quadrangulum, subtus admodum dilatatum, angulo infimo postico perquam prosiliente, septimun, octavun, nomm, decimun quadrangula, versus ultimun magnitudine magis magisque decrescentia, septimum sexto minns; mentun linea longitudinali perquam profunda exaratum; scuta mentalia anteriora magna ovato-lanceolata, latere externo postico oblique truncata, postica anticis duplo minora subtrigona; squamae gulares oblongae, angustae, in utroque apice subtrigonae; dentes ut in congeneribus lormati validi. 'Truncus elongatus, fusiformis, compressus, dorso carinato-compressus, abdomine rotundatus, squamarmm seriebus 21; squamae onnes laeves, lanceolato-imbricatae, spiuae dorsi magnae regnlariter hexagonae; hae squamae versus oeciput magnitudine magis magisque decrescunt et sub forma ovatosubhexagona apparent; squamae occipitis ipsius rero subparvae sunt el fere circulares; squamae abdominalibus scutis contines reliquis parum majores oblongo-rhombeae; scuta abdominalia latiuscula, numerosa; scutum anale integrum; canda tenuis mediocris, teres, superius medio obsolete acuninato-compressa, acuta, squamis hexagonis, scutis inferioribus omnibus medio divisis. Scuta abdominalia $223-225$, scula in fracaudalia $106-107$

Color. Caput, truncus et cauda aterrima, egregie cyaneo-resplendentia; scuta marginalia maxillae et mandibulae flavae pone nigro-marginatae; capitis pars inferior ac gula inmaculate aureo-flavae; truncus a nucha usque ad caudae basin pictus est ad latera fasciis aureo-flavis 36 - 40 angustis, margine subdenticulatis, in spina dorsi diremtis, ibidem versus truncum posteriorem interdum subconfluentibus, ommibus versus scuta abdominalia paullo latioribus haec attamen non cingentibus sed solum latere externo attingentibus, interstitiis latis atro-cyaneis distantibus; truncus subtus ater, paullo olivaceo-lavatus, immaculatus, excepto antico guttis maculisque aureo-flavis, cum fasciarum lateralium basi plus minusve cohaerentibus variegato; cauda prino fasciis lateralibus, dein annulis aureo-flavis, pagina inferiore latioribus ac interdun inter se linea longitudinali, e guttis magnis concoloribus conflata, connexis, variegatur: apex extimus niger est; lingua apice flavido-alla; irides...?

Proportio corporis partillm e mensura. Longitudo a rostri apice usque ad caudae finem 5 $3 \%$, caudae solins 12", capitis 1" 10"; ambitus capitis immediate pone nares 2" 1“", pone oculos 3", occipitis 3\%", colli supremi $23 \% "$, trunci in medio 4", prope anum 2\%", caudae ad basin

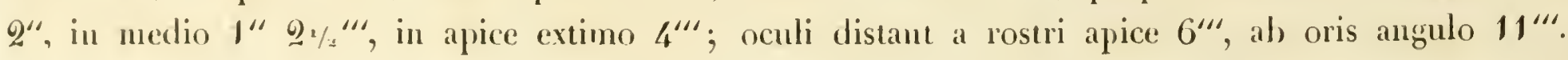

llabitatio et vitae ratio. Habitat vulgatissima in insula $\mathrm{J}$ ava in arboribus, inquilinis admolum venenata falso hahita; victitat nammalibus parvis.

OBSERVATIONES. Specimina Dipsadis dendrophilae asservantur in Museo Lugdunensi Batav., Parisiensi, Monacensi ac in collectione mea; in spiritu vini solum color aureo-flavus in flavido-album aut in albun vergit. In ventriculo speciminis a me dissecti inveni Murem decumanum ab europaeo mullo modo dicersum. 


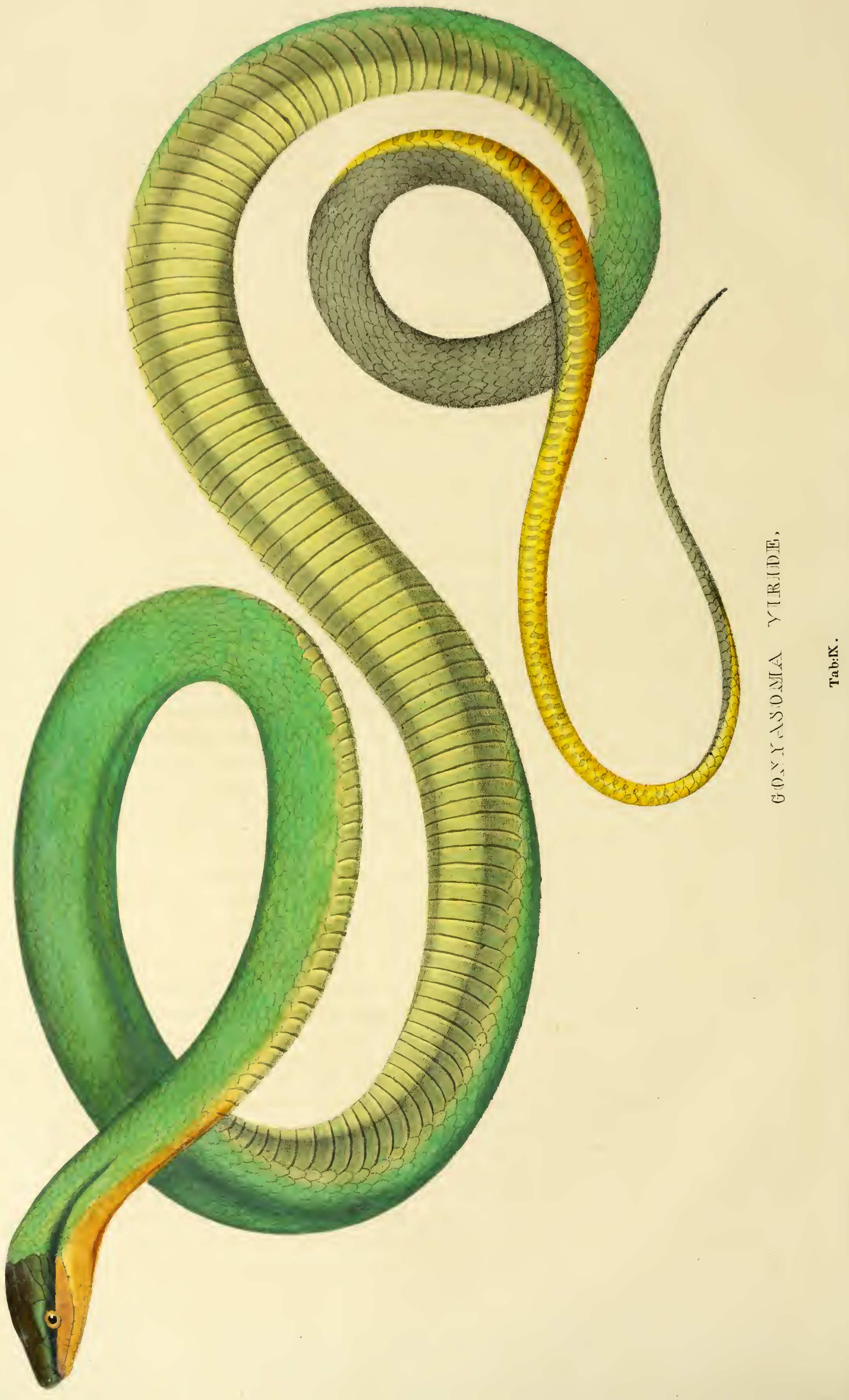




\title{
GON Y OSOMA VIRIDE.
}

\author{
$\begin{array}{lllllllllllllllll}G & \mathbf{R} & \mathbf{U} & \mathrm{N} & \mathbf{E} & \mathrm{R} & \mathrm{K} & \mathbf{A} & \mathbf{N} & \mathrm{T} & \mathrm{E} & \mathrm{N} & \mathrm{B} & \mathrm{A} & \mathbf{U} & \mathrm{C} & \mathrm{H} .\end{array}$
}

'T A в. IX.

Character essentialis. Trunco longissimo, fusiformi, compresso, squamis laevibus tecto, viridi supra, unicolore, flavido-virescente subtus, immaculato; capite elongato-lanceolato, rostro subproducto, obtusiusculo, supra viridi, maxillae lateribus et subtus flavo; vitta a naribus per oculos usque ad occipitis latera ducta obscura, subobsoleta; cauda gracili longissima, subpentagona, supra pallide griseo-olivascente, subtus flava, pallidissime olivascenti-nebulata. Scut. abd. 237, scut. infracaud. 138.

Synonyia: Seba Thes. 2. t. 83. f. 1. (fig. satis a ccurata.)

\section{E S C R I P T I O.}

Corporis forma universalis. Caput subdistinctum, longissimum, obtuso - lanceolatum, depressum, planum, parum altum, supra scuta marginalia labii maxillae lineari-impressum, angustiusculum, ab occipite usque ad rostri apicem magis magisque attenuatum, superius ab oculis versus rostrum vix declive, lateribus, praesertim ante oculos, angulosum; mandibula maxillâ multo brevior; cutis gularis sublaxa; rostrum elongato-productum apice subintrorsum truncato obtusiusculum, labiis vix prominulis verticaliter descendentibus; rictus rectiusculus; nares laterales rostri apici admodum approximatae, majusculae, antrorsum spectantes, subrotundae, in margine antico impressiusculo scuti nasalis sitae; oculi laterales, fere in medio capitis lateralis siti, majusculi, rotundi, superius scuto superciliari non adumbrati, subprominuli; scuta: scutum rostrale majusculum, altum, juxta scutum nasale anticum sinuatum, versus scuta frontalia antica attenuato-subtrigonum, supra marginem basalem profunde lunulato-impressum; scuta frontalia antica duo majuscula pentagona extus versus scutum nasale anticum, oblique pone recte truncatum, scuta frontalia posteriora duo maxima, paullulum versus faciei latera declinata sexangula, attamen antice et postice recto-truncata, solum postice latere externo oblique truncata; scutum verticale maximum, campanuliforme, antice latissimum, lateraliter subemarginatum, apice subangustato trigonum; scuta occipitalia maxima extus oblique marginata ac medio subsinuata, apice postico trigona; scutum nasale anticum majusculum quadrangulum, angulo supremo antico modice ascendente, postice medio naribus subemarginatum, posticum antico paullo minus, pone rotundato-terminatum; squamula elliptica, subobliqua scutum hoc nasale inter et oculare intermedia, stricta, margine scuti frontalis postici deorsum flexi munita; scut um oculare anticum unicum maximum, quadrangulum, angulo supremo externo acutissimo prolongato scuti verticalis basin attingente; scuta ocularia postica duo, parva, superius inferiore paullo majus, quadrangulum, inferius longiusculo-quadrangulum; scuta superciliaria magna, trigona, antice acuminata, pone suboblique truncata absque margine supra oculos prosiliente, plana; scutum temporale verum nullum sed scuta occipitalia latere externo scutellis tribus munita, quorum duo antica aequali fere magnitudine quadrangula, tertium longiusculum, polygonum; scutellum aliud inter scutorum occipitalium apicem posticum, scutella inter nunc nominata et scuta marginalia labialia quatuor, quorum duo inferiora majora pentagona, superiora obsolete pentagona; scuta marginalia labii superioris 9, antica parva, postica sensim majora, antica 5 sibi perquam approximata ac angusta irregulariter quadrangula, multo altiora quam lata, quartum perquam angustatum, sextum quadrangulum aeque altum ac latum, majusculum, septimum sexto multo altius sed non latius, juxta oculum rotundato-emarginatum, octavum septimi altitudine eodem vero paullo latius, quadrangulum, ultimum omnium longissimum, perquam elongatum, longiusculo-quadratum, pone subrotundatum; scutum labiale medium subparvum trigonum; scuta labialia

Wagern, A MPGib. I. 
accessoria lanceolata medio duplo majora; scuta marginalia labii inferioris 12, ulima duo potius squamulae, tria antica angusta, fere aequali magnitudine, quadrangula, quartmon et quintum ejusdem formae, longiora, sextum omnium maximum, magum, pone trigono-terninatun, septimum sexto minus ohliquo-quadrangulum, octavin et nomun ejusdem formae, septimo tamen paullo breviora, decimum longiusculo-(puadrangulum, parum altum; scuta mentalia anteriora ingentia, oblonga, autice subtrigono-ol)tusata, pone latere externo suboblique truncata, longitudinali linea perquam profunda inter se distantia; scuta mentalia posteriora parva, quadruplo inticis minora, pone subovata; squamae gulares oblongae, onnes aequali magnitudine; dentes numerosi, acutissini, mediocres, maxillares postici anticis vix longiores; palatini fere maxillarinn longitudine. Truncus longissimus, admodum fusiformis, perquam compressus, gracilior, in spina dorsi subcarinatus, subtus a gula usque ad anum in utroque latere distinctissime angulosus; canthus, abdomen angulosum formans, scutorum abdominalium flexura rectangula efficitur; squantarum series 25, squante totius trunci ac caudae laeves, mediocres, adpressae, trunci antici et medii subrhombeo-lanceolatae, postici rlombeac, caudac trunci illis majores, basi rhombeae, versus apicem regulariter hexagonat; squamae scutis abdominalibus proximae reliquis vix majores sul_rhombeo-ovales; scuta abdominalia numerosissima perquan ingusta, lata et ultra flexuram multum versus squanas confines extensa, anale medio fissum; cauda continua, longissima, gracilis, sulppentagona, subtus perquam applanata, apice acuta, scutis inferioribus omnibus divisis. Scuta abdominalia 237, scutella infracaudalia 138.

Color. Caput supra ac totus truncus supra et ad latera laetissime viridia, immaculata, latera trunci paullo pallidiora, vertex paullo in olivaceum vergens; cauda supra et ad latera olivascenti-grisescens subtus vitellino-flava, prope scutellorum juncturam subtilissime olivascenti-nubilata; maxillae margo llavus; vitta a naribus per oculos versus occiput ducta olsscure virescens, subolssoleta; mandiloulae margo, mentum, gula ac abdominis pars suprema vitellino-flava, imnaculata, reliqua abdominis pars flava, subtiliter virescenti-lavata, praesertim ad latera, immaculata, solum scuta abdominalia margine apicali strictissimo pellucido subrufescenti-apparente; faux incarnata; lingua nigricans; irides ..?

Proporlio corporis partium e mensura. Longitudo a rostri apice usque ad caudae finem 5 ' 2", caudac solius 17" 2", capitis 1" 7"“; ambitus capitis immediate pone nares 1\%", pone oculos 2"

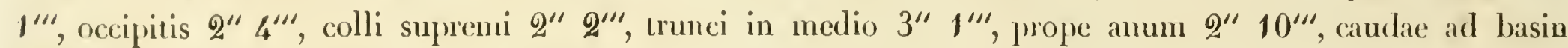

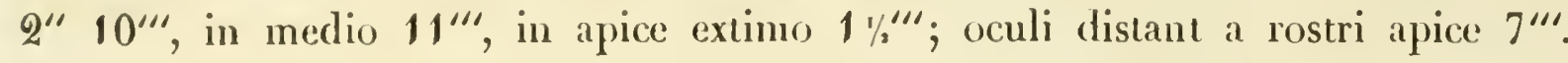

Habitatio et vitae ratio. Habitat in Brasiliac provincia Bahia.

OBSERVATIONES. Specimen depictum et descriptum, recentissime e Brasilia allatum, egregie conservatum adest in collectione mea; aliud nec in Museo Parisiensi nec in Lugdunensi Batav. observavi. Auctorum Coluber viridissimus (scut. abdom. 217, caud. 122), Coluber coerulescens (scut. abd. 215, caud. 170), Coluber pileatus NEUw. (scut.abd. 188, cand. 100) et Coluber herbens NEUW. (scut. abd. 192, caud. 75) colore similes, non solum capilis ac trunci forma toto coelo differunt, sed yuo. que numero scutorum abdominalium ac caudalium. Nullam descriptionem nosco in hoc anuphibium prorsus quadrantem, el SCHEUCHZERI solum icon, in operis sui labula $717 . f .2$. exhibita, ab aucloribus ad Colub rum fulgidum cilata sicut $S_{E B A E}$ cilata icon huc ponenda erunt. Haec $S_{E B A E}$ icon, melius adhuc illius descriptio exhibent satis accurate squamarum structuram, quam in figura nostra, quoad caudae superiorem partem, parum artifex respexit. - Capitis forma et praecipue structura particulari anticorum scutorum marginalium labii superioris strictissimorum, scutis maximis mentalibus anticis, scutello elliptico scutum oculare anticum inter et nasale posticum intermedio nec non abdominis perquam angulosi habitu haec species facile distingrienda erit ab omnibus simili modo tinctis. Trunci superioris color speciminis in spiritu vini asservati in dilule viride, inferioris in sordide flavum vergit; epidermide detrila truncus superius coeruleus apparet squamarum marginibus nigris, cauda albida, caesio-nubilata. 



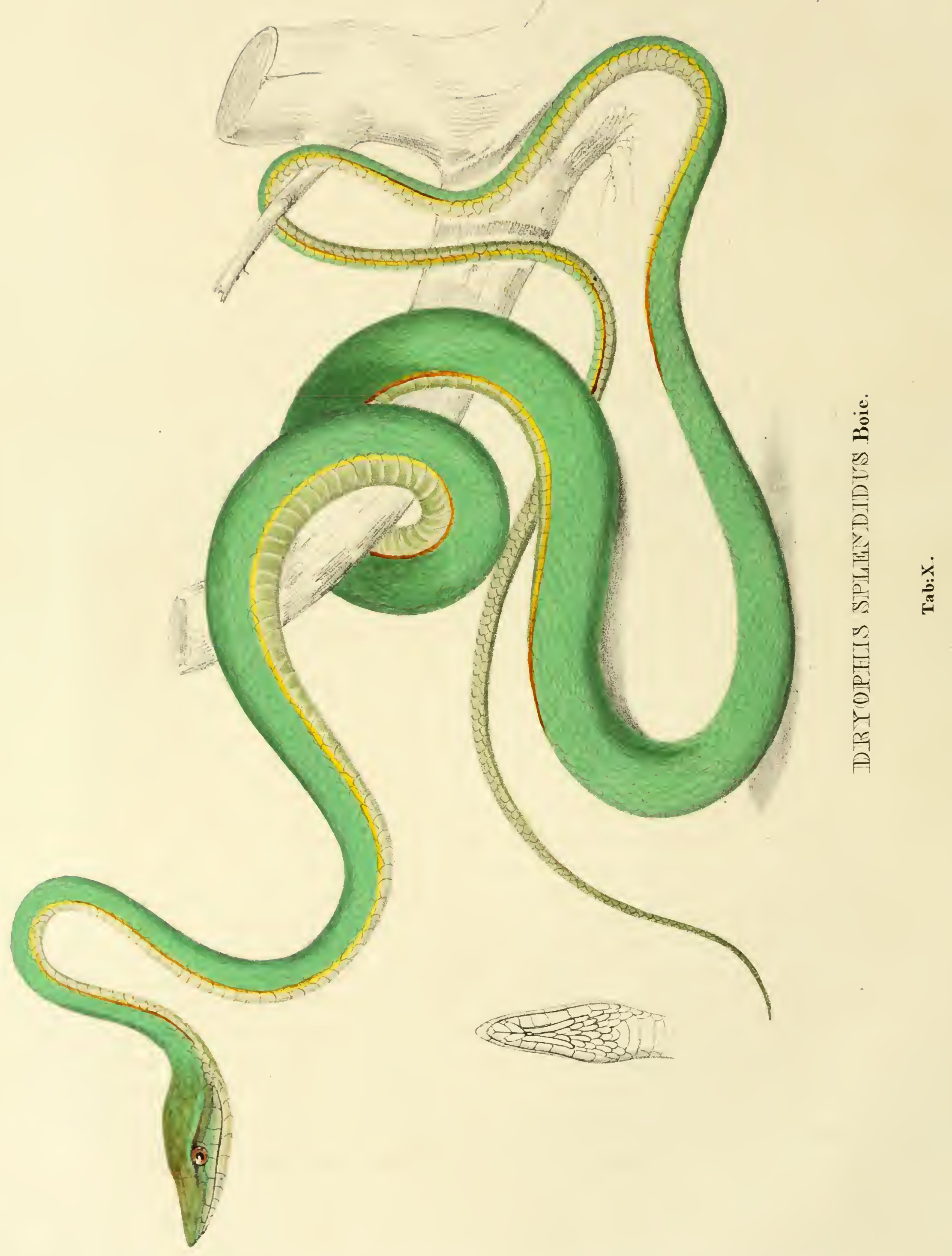




\title{
DRYOPHIS FULGIDUS Boie.
}

\author{
B L I N K E N D E B A U M N A T T E R.
}

T ^ в. $\mathrm{X}$.

Character essentialis. Capite elongato tetragono-pyramidali rostro elongato, trigonolanceolato, recto, mobili; trunco longissimo, gracili, fusiformi; squamis lanceolatis, dorsi medii carinatis, laterum laevibus; cauda longissima, continua, subtus plana, acuta, squamis rhombeo-hexagonis, laevibus, solum anticis mediis carinatis; capite, trunco et cauda supra laetissime viridibus, unicoloribus; maxillae lateribus, capitis, trunci caudaeque latere inferiore laete virescentibus, unicoloribus; linea rectissima utrinque a gulae lateribus usque ad caudae finem juxta scutorum abdominalium latera externa ducta, aureo-flava, versus caudae finem magis magisque obsoleta. Scut. abd. 197, scut. infracaud. 130.

Symonyia: Coluber fulgidus Daud. Rept. 6. p. 352. t. 80. (descriptio non satis accurata, icon mediocris) Dryoph is fulgidus Bore in litt.

\section{E S G R I P T I O.}

Corporis form a universalis. Caput distinctum, longissimum, angustatum, telragono-pyramidali-acuminatum, ante oculos paullo latius quam altum, pone hos fere aeque altum ac latum, supra occiput convexiusculum, supra verticem et frontem applanatum; rostrum longissimum, lateribus applanatum, superius devexiusculo-depressum, apice prolongato, recto, trigono-lanceolato acuminato, basi latiusculo, non subito attenuato, mandibulae apicem multum excedente, plano, mobili; mandibula maxillâ multo brevior apice labii trigono-rotundato subprolongato, mobili, plano; oris angulus subarcuato-ascendens; nares laterales rostri mobilis basi sitae, mediocres, rotundatae, retro spectantes; oculi laterales, subobliqui, majusculi in nedio capitis lateralis siti, ergo a rostri apice largissimo interstitio diremti, scuto superciliari margine externo paullo prominulo parum adumbrati; scuta: scutum rostrale inferum, lanceolatum, in utroque baseos latere recto-abscissum; scuta frontalia anteriora majuscula subirregulariter trigona, multo longiora quam alta, pone suboblique truncata; scuta frontalia posteriora anticis triplo fere majora, maxima, latere externo usque ad scuta marginalia labii superioris rectangulo-deflexa, pone, juxta aciem externam, anguloso uni-emarginata, juxta scutum verticale recto-, prope scuta frontalia anteriora subtrigono-terminata; scutum verticale campanuliforme, longiusculum, subangustum, pone trigono-rotundatum; scuta occipitalia majuscula, latere externo suboblique truncata, antice paullo bi-emarginata; scutum nasale anticum admodum pusillum, regulariter trigonum; scutum nasale posticum (ad cujus marginem anticum nares sitae) parvum, antico parum majus, rhombeum (scutum inter hoc et oculare anticum intermedius scutorum nasalium posticorum margo latus deflexus, ergo scutum loreum nullum); scutum oculare anticum unicum majusculum cum margine sursum flexo scuti superciliaris et nasalis postici apicem attingente trigonum, latere antice et postice subtiliter arcuato-emarginatum; scuta ocularia posteriora tria (an duo?) parva, quadrangula; scuta superciliaria majuscula scuti verticalis magnitudine, postice rotundato-, antice subattenuato-trigono-terminata; scuta temporalia tria, quod pone scuta ocularia magnum, dilatato-trigonum aut potius trigono-pentagonum, duobus subsequentibus minoribus subrhombeis; scuta marginalia labii superioris 10 , ab oculo versus rostrum magnitudine sensim decrescentia, subparva, primum longiusculo-subtrigonum, 2, 3, 4, 5, 6 quadrangula, septimum fere quadrangulum angulo postico supremo subtruncato, octavum omnium maximum, magnum, quadrangulum, nonum octavo dimidio minus altum quadrangulum, ultimum quadrangulum, praecedentis longitudine eodem autem di- 
midio fere minus altum; scutum labiale medium parvum, trigonum; scuta labialia accessoria mediocria, longiusculo-ovata, pone acuminata, scutorum mentalium anticomm magnitudine el forma; scuta manginalia labii inferioris 10, dno ultima vix senta potius squamae parvae, reliqua ommia subobliquo-quadran-

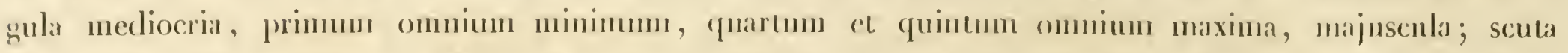
mentalia anteriora seutorum labialium accessoriormm magnitudine et forma muiversali, allamen antice magis attenuata, postice squammla ovali oblonga munita; seuta mentalia posteriora longissina, strictissima, subarcuato-linearia, ambo simnl combemplata fere )(-formia, medio stpamulan strictissinam, dongatam includentia; squamae gulares imbricatac laeves; dentes mediocres, acutissimi, maxillac tres postremi validi omnimn maximi. 'T' r u planiusculo-rotundatus, senamarum seriebus 17; squamae trunci superioris lanceolatae, versns caudam subrhombeo-lanceolatie, laeves, dorsi medii per series septem carinatae, in ultima utrincpue serie subobsolete carinatae, caudae rhombeo-hexagonae trunci illis latiores, laeves, exclusis anticis mediis subcarinatis; squanue scutis abdominalibus proximac, reliquis paullo majori, rhombeo-lanceolatie; scuta abdom inalia mmnerosa, angustiuscula, anale medio fissm ; caud a continua longiuscula, gracillima, teretiusculo-subangnlosa, subtus planiuscula, apice acuta, scutis inferioribus omnibus divisis; ani orificiun utrinque scutellis quatemis munitum quormm tria antica parva aequali latitudine, quartum anticis triplo majus transversim ellipticum. Scuti abdominalia 197, scutella infracaudalia 130.

Color. Caput, truncus et cauda supra lactissime viridia, immaculata; capitis partes laterales ac inferiores, totus truncus inferior nec nou caudae pagina inferior lacte virescentia, immaculata; linea utrinque a gulac latcribus usque ad caudae funem juxta scutorum abdominalium latus externum ducta rectissima, aureo-flava, attamen versus caudae finem magis magisque obsoleta; lingua virescens; irides..?

Proportio corporis partium e mensura. Longitudo a rostri apice usque ad caudae finen 4" 2\%", caudae solius 17", capitis 1" 5"'; ambitus capilis immediate pone nares 101/2", ponc oculos 1"7"“, occipitis 1" $8^{\prime \prime \prime}$, colli supremi $1^{\prime \prime} 4^{\prime \prime \prime}$, trunci in medio $2^{\prime \prime} 2^{\prime \prime \prime}$, prope anum 1" $3^{\prime \prime \prime}$, caudae ad basin $1^{\prime \prime} 3^{\prime \prime \prime}$, in medio 7\%",", in apice extimo vix 3"'; oculi distant a rostri apice 7"“; latitudo capitis supra oculos $44^{2 / 4}$ ", supra nares $2^{\prime \prime \prime}$; rostri apex mobilis $13^{3 / 4}$ " longus, basi $2^{\prime \prime \prime}$ et apice $3 / 4^{\prime \prime \prime}$ latus est.

Ilabitatio et bitae ratio. Habitat in Brasiliae provincia Pará in arboribus, et, ut dicitur, in Antillis insulis.

OBSERVATIONES. DAUDINI descriptio parum accurata non tangit dorsi medii squamas carinatas quam ob rem pro aliena eam vendidissem, nisi ipse observassem specimen ab, illo descriptum in Mnseo Parisiensi asservatum. Coluber fulgidus MERREMII cum DAUDINII Colubro fulgido unitus differt toto coelo; anctoris descriptio exhibet speciem in Java insula vulgatissimam, ab amicissimis REINWARDT et BoIE „Dryophis rostratus" denominatam. - SHAWII Coluber purpurascens a MERREMIO citatus et a Dryophe rostrato et ab hac specie prorsus distinguendus est. Dryophis prassinus REINwandTI (SCHEUchz. Phys. Sacr. t. 630. f. A. SEBA Thes. 2. t. 53. f. 4. (Coluber nasutus SHAr), Russ. Ind. Serp. 2.p. 28. t. 24, species similis est, attamen squamis onnino laevibus facile ab hac discernenda. - Scutorum ocularium posticorum humerum verum ignoro; in specimine Musei Monacensis unum capitis latus monstrat scutella tria, alterum duo solnm. - In spiritu vini hujus egregii serpentis color ad sordide viride, linea lateralis ad album inclinat; epidermide detrita truncus superior in cyaneum vergit. - Pro Dryophe splendido tabulae nostrae per errorem adjecto, lege: Dryophis fulgidus. 



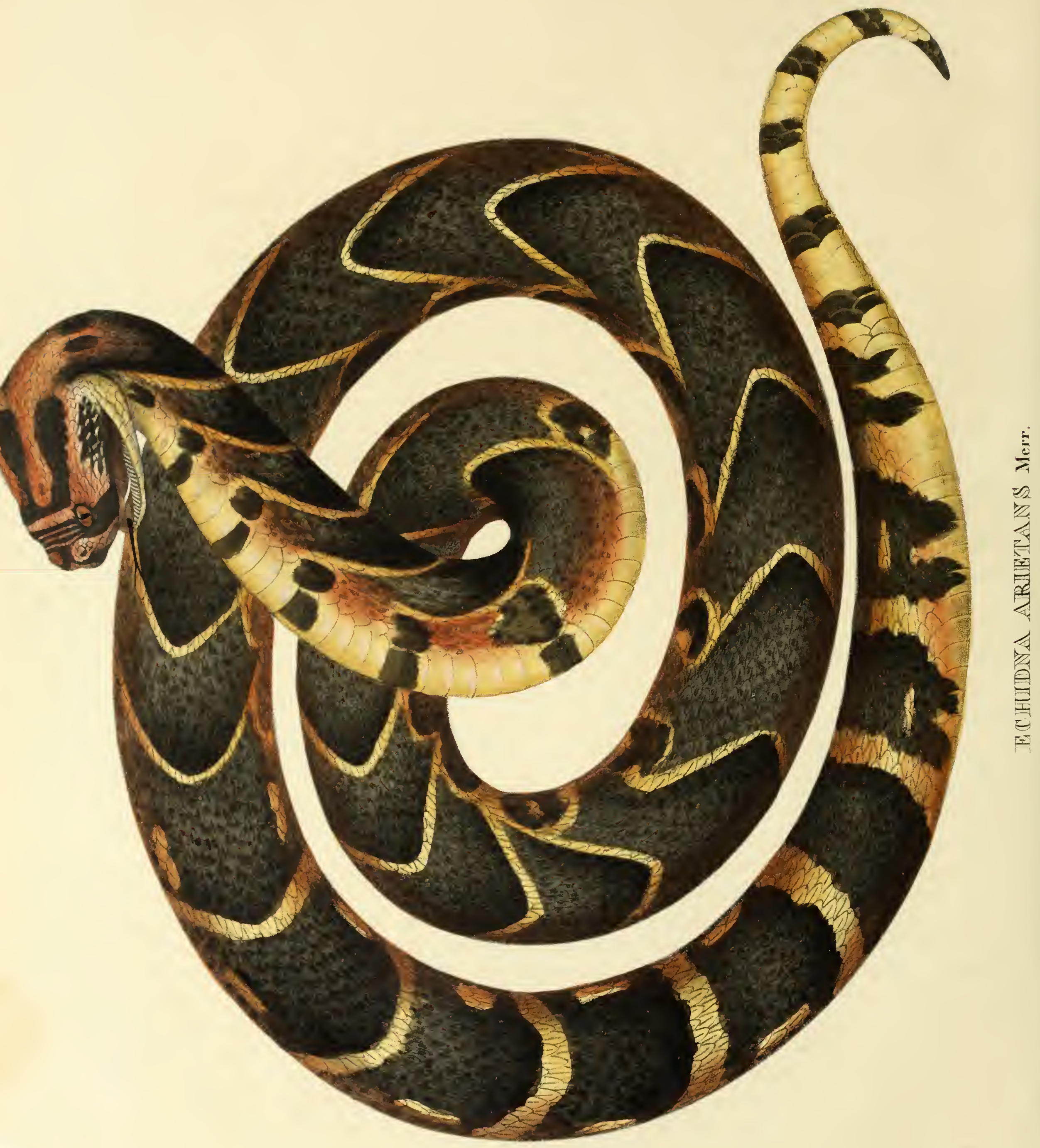




\title{
E C H I D N A A R I E T A N S Merr.
}

\author{
$\begin{array}{llllllllll}\mathrm{P} & \mathrm{U} & \mathrm{F} & \mathrm{F} & - & \mathrm{O} & \mathrm{T} & \mathrm{T} & \mathrm{E} & \mathrm{R} .\end{array}$
}

T A B. XI.

Character essentialis. Naribus magnis superius menbrana rugosa, integra cinctis; squamulis inter illas intermediis duabus margine externo sursum flexis; scutis mentalibus anticis maximis; trunco incrassato, subbrevi; cauda bresissima conico-terminata; squamis trunci, caudae capitisqué ovato-lanceolatis admodum carinatis; capite supra, trunci candaeque lateribus testaceo-rubris; fascia supra frontem per oculos ducta, taenia verticis gemina longitudinali antice connata, altera parallela supra oris angulum ducta nigricantibus; dorso nigricanti-, aut cinereo-fusco, antico maculis circiter 19 magnis trigonis, nigris, pone flavido-rubicundo marginatis, lateraliter macula concolore utrinque munitis, dorso postico fasciis latissimis nigricantibus, lateraliter utrinque lineola horizontali flavo-rubicunda notatis obtecto; gula flavida; trunco ac cauda subtus flavidis, illo taeniolis transversis per magna interstitia nigris notato, hac subtus nigricanti-nubilata, supra fasciis latis nigricantibus ac strictis testaceo-rubris signata. Scut. abdom. 136-150, scut. infracand. 20-27.

Synomyin: Seba Thes. 2. t. 30. f. 1. (Fig. bona; Vipère à courte queue Cur. R. a. 2. p. 84. Coluber Lachesis var. Shaw Gen. Zool. V. 3. P. 2. p. 403. t. 106. quae tabula Sebana) t. 54. f. 4. (Fig. satis accurata a Gmelin et Daudin falso inter synonyma Xenodontos severi Borer recepta; Coluber Bitis Bonvat. Oph. p. 22.) t. 93. (fig. pessima; Colu ber Clo тho Guel. Syst. Nat. p. 1086.) t. 94. (fig. mala; Coluber Lachesis Gmel. Syst. Nat. p. 1085 et Shaw 1. c. p. 402. t. 105. quae tabula Sebana.) Echidna arietans Merr. Tent. Syst. Amph. p. 152. n. 12. Coluber hebraicus Lacér. Quadr. ov. 2. p. 106. Vipera hebraica Latr. Rept. 3. p. 335.

\section{E S C R I P T I O.}

Corporis forma universalis. Caput distinctum, depressum, trigono-obtusatum, latum, superius planum, lateribus devexum, ante oculos paullo angulosum, apice truncato-obtusato impressiusculum; oris rictus amplissimus angulo rectiusculo; nares magnae, superae, rotundatae, antrorsum spectantes, membrana plana, subplicata, integra limbatae, alia interius margine postico plica subbursaria exstructa semi-expletae, oculis aeque approximatae fere ac rostri apici; oculi laterales attamen frontis margini admodum approximati, mediocres, subobliqui, longiusculo-rotundati, rostri apici duplo propiores quam oris angulo; scuta capitis superioris praeter marginalia labialia vera omnino nulla, scutum rostrale majusculum pone subtrigono-terminatum; scutella labii superioris 15, omnia quadrangula, quatuor antica reliquis paullo minora, reliqua aequali fere magnitudine, plana; squamae capitis supremi ac lateralis omnes ovatae, perquam carinatae, et praeterea, accuratius contemplatae, rugosae, exceptis squamulis oculum cingentibus ovato-rotundatis, aequo modo rugosis ac formatis; inter nares adsunt squamulae duae oblongae margine externo sursum adscendentes, quae sequuntur 5 ovatae, carina maxime elevata praeditae; squamulae immediate pone scutum rostrale 8 fere in morem flosculi collocatae subpentagonae, admodum rugosae; squamae scutellis labialibus proximae reliquas capitis magnitudine superant, attamen earum forma gaudent; scuta marginalia labii inferioris 14 , mediocria, tria antica reliquis majora, tertium omnium altissimum, angustum, quadrangulum, duo antecedentia ejusdem formae, reliqua aequali fere magnitudine, quadrangula, exceptis scutellis 4, 5, 6, 7, 8 inferius subtrigono-terminatis; scutum labiale medium latiusculum regulariter triangulum; scutum labiale accessorium elongatum, apice infimo 
trigonum, margine externo augulo in medio prominulo insigne; scuta mentalia anteriora maxima subcordiformi-pentagona; scuta mentalia posteriora antecedentibus duplo minora ovato-elliptica; capitis inferioris pars reliqua obtegitur squamis subovatis, laevibus, quarum tres pone scutellum labiale tertium incipientes omminm maximae, majusculae, praesertim intermedia, polygonae; squamae quoque scutellis labialibus proximae subhexagonae sunt; dentes ut in reliquis congeneribus formati, validi; telum, in utroque maxillae latere unicum, ingens, arcuatum. Truncus breviusculus, torosus, fusiformis, teres, abdomine rotundatus, squanarum seriebus pone collum 29; squama e omnes carina perquam prominente, acuta, solum in squamarum serie scutis abdominalibus proxima minus prominula, interdum obsoleta, munitae, ovato-lanceolatae, ultimae utrinque seriei reliquis paullo latiores, apice magis obtusae; scuta abdominalia breviuscula, lata; scutum anale margine rotundatum integrum; cauda brevissima, crassiuscula, teres, conico-acuminata, apice non in aculeum terminata, superius squamis lanceolatoovatis, perquan carinatis, subtus scutellis pentagonis obtecta. Scuta abdominalia 136-150, scuta infracaudalia $20-27$.

Color. Caput supra, trunci caudaeque latera testaceo-rubra; fascia supra frontem per oculos usque ad maxillae marginem ducta lata nigra, in fronte lineis quatuor testaceo-rubris dissecta; taenia supra oris angulum horizontalis, versus oculum directa, nigra; taenia verticis transversa nigra, latiuscula, ex utroque apice linean latiusculam, concolorem, usque ad occiput porrectam emitters; regio ante et inter nares paullo nigro-variegata; scutella marginalia labii superioris in fundo flavido testaceo-rubicunda, labii inferioris, mentum ac gula flavida; dorsum nigricanti-, aut cinereo-fuscum, anticum maculis circiter 12 magnis, longitudinalibus, trigonis, nigris, pone linea flavido-rubicunda, margine subdenticulata distinctissina laete marginatis, lateraliter utrinque cum macula concolore, ipsis triplo minore, trigono-rotundata connexis notatum, posticum variegatum fasciis latissimis, interstitiis angustiusculis testaceo-rubicunlis diremtis, nigricantibus, in utroque latere inferius emarginatis, ante apicem lateralem lineola latiuscula, longitudinali, testaceo-rubicunda notatis; cauda supra nigricans fasciis angustiusculis testaceo-rubicundis quatuor dissecta; truncus et cauda subtus flavida, ille fasciolis brevibus, vix usque ad medium scutum abdominalis ductis, alternantibus, magnis interstitiis distantibus, nigris notatus, haec, praesertim apice, paullo testaceo-rubicundo lavata et maculis subobsoletis nigricantibus nubilata; irides...?

Proportio corporis partium e mensura. Longitudo a rostri apice usque ad caudae finem

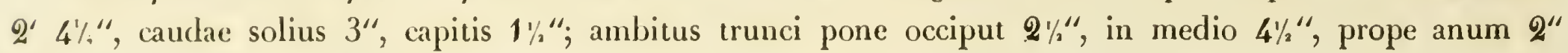
$2^{\prime \prime \prime}$, caudae ad basin $1^{\prime \prime} 8^{\prime \prime \prime}$, in medio $1^{\prime \prime} 4^{\prime \prime \prime}$, in apice extimo 10"' oculi distant a rostri apice 4", ab oris angulo $8 \%$.

Habitatio et vitae ratio. Habitat in Africa meridionali, in Promoutorio bonae spei satis frequens, ac, ut dicitur, in Senegambia.

OBSERVATIONES. Specimina Echidnae a rietantis asservantur in Museo Parisiensi, Lugdunensi Batav.; duo adsunt in collectione mea, quorum unum 4\%"longum, et in trunci medio $5^{\prime \prime}$ latum est. Speciem hanc Senegambiam quoque inhabilare audivi ex navilis qui ibi maxime detestatam magno numero offendisse tradidere. - 



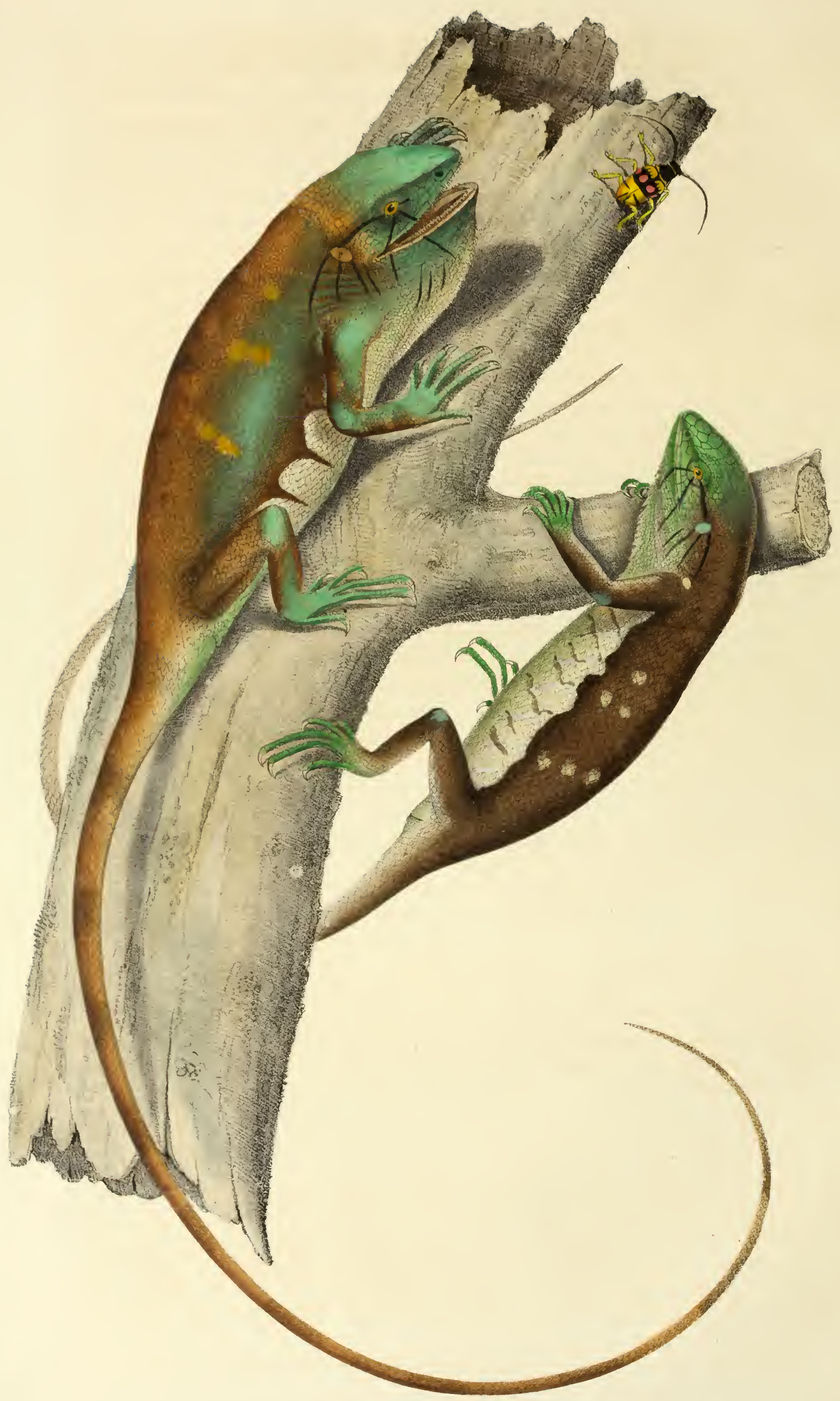

PORYCHARTS YIRESSCHYS NeUW.

Tiab:XII 


\title{
POLYCHRUS VIRESCENS.
}

\author{
$\begin{array}{llllllllllllllllllll}G & R & U & N & L & I & C & H & E & F & A & R & B & E & R & E & C & H & S & E\end{array}$
}

T A в. XII.

Character essentialis. Gulae plica mediana denticulata; femoribus absque poris veris; capite tetragono-pyramidali, obtuso, pagina superiore applanato; cauda tereti truncô triplo longiore; capitis parte maxima, digitis, cubito ac genibus laete virescentibus; partibus pedum ac trunci inferioribus albis, fuscescenti-, ani regione ac abdomine medio virescenti-lavatis; corporis partibus superioribus rufo-fuscis lateribus ex flavo ac viridi variegatis; oculis lineis tribus nigris percussatis.

Synonyma: Polychrus virescens Prinz von Neuwied in Schinz Uebers. des Thierr. von Cuv. Bd. 2. S. 65. Id. Polychrus inarmoratus Beytr. zur Naturg. Brasil. Bd. 1. S. 110 et Spix Lacert. brasil. t. 14. (icon e specimine decolorato.)

\section{E S G R I P T I O. $\left.{ }^{*}\right)$}

Corporis forma universalis. Caput majusculum tetragono-pyramidale superius ab oculis ad rostri apicem admodum declinatum, lateribus perpendicularibus planum, pagina superiore latiuscula non impressum, irregularibus scutis majusculis, margine non prominulis praesertim a rostri apice usque ad oculos obsitum, apice multo latius quam altum, obtusum, depressum, ante aures intumidum; mandibula quam maxilla paullo longior apice rotundata; nares a rostri apice subamotae, subobliquae, majusculae, antrorsum spectantes; oculi parvi, cute granulato-squamulosa, ut in Chamaeleonte convexo-prominula, horizontaliter parum fissa, inclusi, occipiti fere propiores quam rostri apici; scuta labii superioris ac inferioris omnia regulariter quadrangula, labii superioris 8, inferioris 9, ultima minima, labii inferioris quam scuta superioris panllo majora, labii superioris margine inferiore prominula, scutum rostrale latum sed parum altum; scuta ante nares duo, anticum parvum irregulariter rhombeum, posticum magnum sublanceolatum; scutum nasale magnum, supra subrotundatum, subtus scuto labiali recto-truncatum, nasi foramen medio gerens; scutella duo immediate pone scutum nasale, inferius superiore subirregulariter quadrangulo longius ac dimidio minus altum, quadrangulum; scutella tria sequentia forma perquam diversa; eorum infimum omnium longissimum, elongatum, in utroque apice subtriangulari-acuminatum, medium, trium minimum, triangulum, supremum subrliombeum; scutella sequentia pone haec scuta palpebras inferius cingentia irregulariter quadrangula, interdum emarginata; capitis pagina superior gerit a scuto rostrali usque ad oculorum medium, inclusis scutellis marginalibus, scuta 23, plana, duo pone scutum rostrale mediocria, rotundato-subhexagona, pone scutum, capitis omnium fere maximum, magnum, scutiformi-subhexagonum, in utroque latere scutello anguloso-ovali munitum gerentia; scutum supra scutum nasale conspicuum subparvum, longiusculo-hexagonum; alia inter haec duo posita tria magna, intermedium laterali pentagono paullo minus regulariter pentagonum, versus rostrum strictius; scuti hujus lateralia margine externo subpostico utrinque scutellum subpentagonum sequitur, pone quod sequuntur supra orbitas scutella duo oblonga, hexagona, quae scuta quatuor includunt magna, excepto medio quadrangulo, pusillo; laterale hujus utrinque hexagonum, posticum, inter quatuor maximum, subseptangulum; pone quod vertex impressiusculus scutellis circiter 20, irregularibus, prominulis obsitus est; scuta capitis reliqua laevia sunt ac neutiquam margine prominula; temporum regio lateralis ac margo supremus squamis polygonis, laevibus, palpebrae squamis minutis subgranulosis, numerosis, cutis gularis squa-

*) Descriptio haec spectat foeminam a me dissectam, ac in tabula 12 arboris in pagina superiore delineatam; figura inferior, minor exhibet verisimile speciem distinctam.

Wabler, AMphib. I. 
mis admodun parvis, subgranuloso-longiusculis et medio antico squamulis longioribus, trigonis, erectis 8 , serrae speciem formantibus olsita; scutmin labiale mediocre in utroque latere semel-, postice bi-emarginatum, medio lineola, marginem anticum non attingente, exaramm; squanae inferiona scua labialia inferius marginantes laeves, hexagonae in duplice serie positae, versus gulan magis magisque magnitudine decrescentes; a 11 res ovales; dentes maxillares ac dentes palatini haud numerosi ut in reliquis congeneribus formati; Iruncus crassiusculus, compressiusculus, altior quan latus, spina dorsi carinato-compressiusculus et subcurvatus, versus fenora angustior; s qu a m a e colli totius pusillae subquadrangulo-granulosac, laterales interjectis mimutissimis, laeves, superiores subcarinatae, pelum, trunci totins ac caudae carinatae, trunci lanceolatae solum in spina dorsi subpentagonae, abdominis perquam acuminatae et carinatae, pedum subrhombeac, digitormu superiores latiusculae, laminiformes, obsolete carinatae, margine antico subfimbriatae, palmae ac plantae pentagonae, punctulo intermedio prominulo exasperatae, digitorm inferiores mmerosac, transversae, granuloso-carinatae, caudae basi rhombeae, versus basin rhombeo-subhexagonae, carina perdistincta; pedes graciliores, postici anticis paullo validiores; femora subtus abs fue poris, horum loco squainulae, reliquis paullo majores, irregulares, convexiusculac, medio inargine utplurimum punctulo prominulo sive impresso munitac; an us transversus margine squanis pusillis, muncrosis, lacvibus, conrexiusculis, obsitus; cauda longissima, gracillima, tereti, solum squamarum carinis angulata, corpore, exclusis collo et capite, triplo lere longior; ungues scansorii, compressi, acutissimi ut in Galeopitheco formati, superius ac inferius a basi usque ad medium squama laevi muniti.

Color. Caput totum, exclusis temporibus in medio ac occipite, colli ac trunci latera, digiti, cubitus ac genu laete ac dilute viridia; trunci latera praeterea maculis laete flavis picta apparent; gula, pedun latus interius ac totum abdomen albida, subfuscescenti-, gnla, abdomen in medio ac anus viridilavata; occiput, tempora in medio, dorsmu totum, pedum pars superior rehicua ac cauda rufescenti-liusca, spina dorsi lasciis nonnullis, obsoletis, cauda annulis obscurioribus ejusdem coloris pictae; trunci latera infra viredinem lusco-variegata nonnullos angulos ejusdem coloris versus abdomen emittunt; tympanum viride; lineae per oculum tres nigrae, suprema horizontalis omnium longissima supra et ultra aures-, sequens oblique versus et paullo ultra oris anguluin, tertia rerticaliter versus maxillam ducta; cutis gularis et colli latera albido subvariegata nomnullis lincolis nigricantibus variegantur; pedes supra fasciis obsoletissimis obscurioribus picti; iricles aureae; ungues albi culmine nigro; lingua et faux incarnatae.

Proportio corporis partium e mensura. Longitudo a rostri apice usque ad caudae finem 21", caudae solius 14\%", capitis $1^{\prime \prime} 44^{\prime \prime \prime}$, brachiii $1^{\prime \prime}$, antibrachii 8“", femoris $\mathcal{1}^{\prime \prime} \mathbf{1}^{\prime \prime \prime}$, tibiae $11^{\prime \prime \prime}$; latitudo capitis immediate ante oculos $6 \%{ }^{\prime \prime \prime}$, supra nares $44^{\prime \prime \prime}$, ejusclem altitudo ante illos 6x/4", ante has $3 \%$ "'; oculi distant a naribus $3 \%$ " a rostri apice $7 \%$,

Habitatio et vitae ratio. Habitat in Brasiliae sylvis, in arboribus, prope Rio de Janeiro ac in provinciis confinibus non infrequens, insectis victitans ${ }^{*}$ ); pulmonibus magnis instructa bestiola haec pulcherrima corporis colorem pro lubitu mutare valet, ita quidem, ut trunci lateralis color fuscus sive viridis $110 \mathrm{x}$ obscurior sit, mox dilutior; iritata gulae cutem in orbem fere expandit; foemina, mense Majo gravida, parit ova 10-12 alla, magnae nucis avellanae forma, tali vero paullo longiora; Brasiliac colonis Camale ão rocatur.

OBSERVATIONES. Num inferior figura minor, in tabula cilata 12 conspicienda, e specimine masculo, Brasiliensi (?) hausta, sexus sive aetatis diversitatem praecedentis, aut speciem distinctam exhibeat, ego, pluribus speciminibus comparationi inservientibus destitulus, discernere non audeo. - Color, quo hoc animal Polychro virescenti proximum, et quo iterum ab eo discedit in collo, dorso ejusque lateribns et in pedibus obscure chocoladino-fuscus est, in dorso supremo fascüs nonnullis dilutioribus, vix conspicuis, in ejus lateribus macnlis subrotundis, per tres series dispositis, haud numerosis, albi-

*) In ventriculo speciminis a me dissecti inveni praeter formicas ac Blattarum residua exemplar paullo mutilatum Lamia e mutilla. riac lírcu, in alio Polychro flores plantae mili ignotae. 
dis variegat us; rostrum, verticis pars, cubitus, genua digitique supra virescunt, reliquae corporis capitisque inferioris partes nec non pedum latus internum albidae sunt, gula et abdomen medium anique regio viridi-lavata; juxta trunci latera conspiciuntur in fundo albo lineae 6-7 subdenticulatae nigricanti-fuscescentes, suboblique antrorsum spectantes et basi utplnrimum sinubus coloris fusci trunci respondentibus; maxillarum margo paullo in flavidum, cauda unicolor in fuscum vergit et superius basi subtiliter virescenti-lavata est; tympanum viride; oculorum lineae nigrae ut in Polych ro virescent e occurrunt, attamen non ultra aures et orem extensae; viridi-marginatae sunt. Gula medio squamuloso-denticulata, femur poris veris privatum est; in hoc solum, pororum loco, squamulae nonnullae reperiuntur reliquis paullo majores, subrotundae, medio punctulo impressiusculo munilae. Pedum, trunci gracilioris, caudae, squamarum, trunci capitisque forma prorsus eadem, quae Polychri virescentis, attamen caput superins scutis minus planis tegitur; ante et pone nares unicum solum scutellum adest, exclusis scutellis duobus immediate ante oculorum cutem conspicuis; reliqua capitis scuta superiora forma parum discrepant. Longitudo a rostri apice usque ad candae finem 18", caudae solius 121/2", capitis 11/4", brachii 3/4", antibrachii 7" , femoris 11" tibiae 3/4"; latitudo capitis immediate ante oculos 6 ", supra nares $51 / 2$ ", ejusdem altitudo ante illos $4 \%$ ", ante has $3^{1 / 2} "$ "; oculi distant a naribus $3^{\prime \prime \prime}$, a rostri apice 6"'. - Polychrus hic, si sane differat a Polychro virescente, nomine adjectivo "strigiventris" designari potest. - Polychrus marmoratus a Polychro virescente capitis structura, femorum poris distinctissimis, colore etc. toto coelo differt; attamen observare debeo femorum poros in designandis speciebus Po lych rorum nullius magni momenti esse et solum secundum vitae stadia (an secundum sexus.?) plus minusve perfectos occurrere videri. Polych ri virescent is specimen vivum, admodum aegrotans, vidi Londini; ejus iridum color in meo itineris diario zoologico aurea vocatur, quam cl. princeps Neovidensis obscuram ait. - SPIXII Poljchrus acutirostris (Lacert. brasil. t. 14 a.) species distincta est, cujus dentium et interna corporis structura sicut externa ut in Polychro virescenti et P. marmorato adest; non foret ideo, cur eum in distinctum genus ,Ecphymotes" (FYTZYNGER Neue Classification der Reptil. S. 17.) secernamus, si nil aliud discriminis inter eos intersit, nisi quod pori femorum adsint sive desint. - Polychrus fasciatus ( $D_{E}$ LA Porte, Annales des scienc. nat. T. 9. Sept. 1826. p. 110) quem Moluccarum Insularuin incolam credunt, species mihi ignota vix hoc genus spectat. Colores virides Poly־chri virescent is in spiritu vini asservati paullo, attamen non subito et prorsus pallescurt, color dorsi rufo-fuscus restat, ejus vero maculae flavae subito albescunt. Specimina descripta adsunt in Museo Monacensi; Polychri virescent is alind exstat in Museo Lugdunensi Batav. 


\section{$\begin{array}{llllllllll}C & O & R & R & \text { I } & G & \text { E } & \text { N } & \text { D } & \text { A. }\end{array}$}

Coloris descríptio jam exhibita Aspidoclonii se in ifasciati falsa, calami lapsu orta, cum sequente e specimine vivo commutari debet.

Aspidocl. seruifasciat. Corpore et cauda albis fasciis, in abdomine apertis, latis nigro-chalybeis ornatis; corporis albedine fasciam primam inter, secundam tertiam et quartan nec non mandibula subtiliter flavo - lavatis. 

3. 3
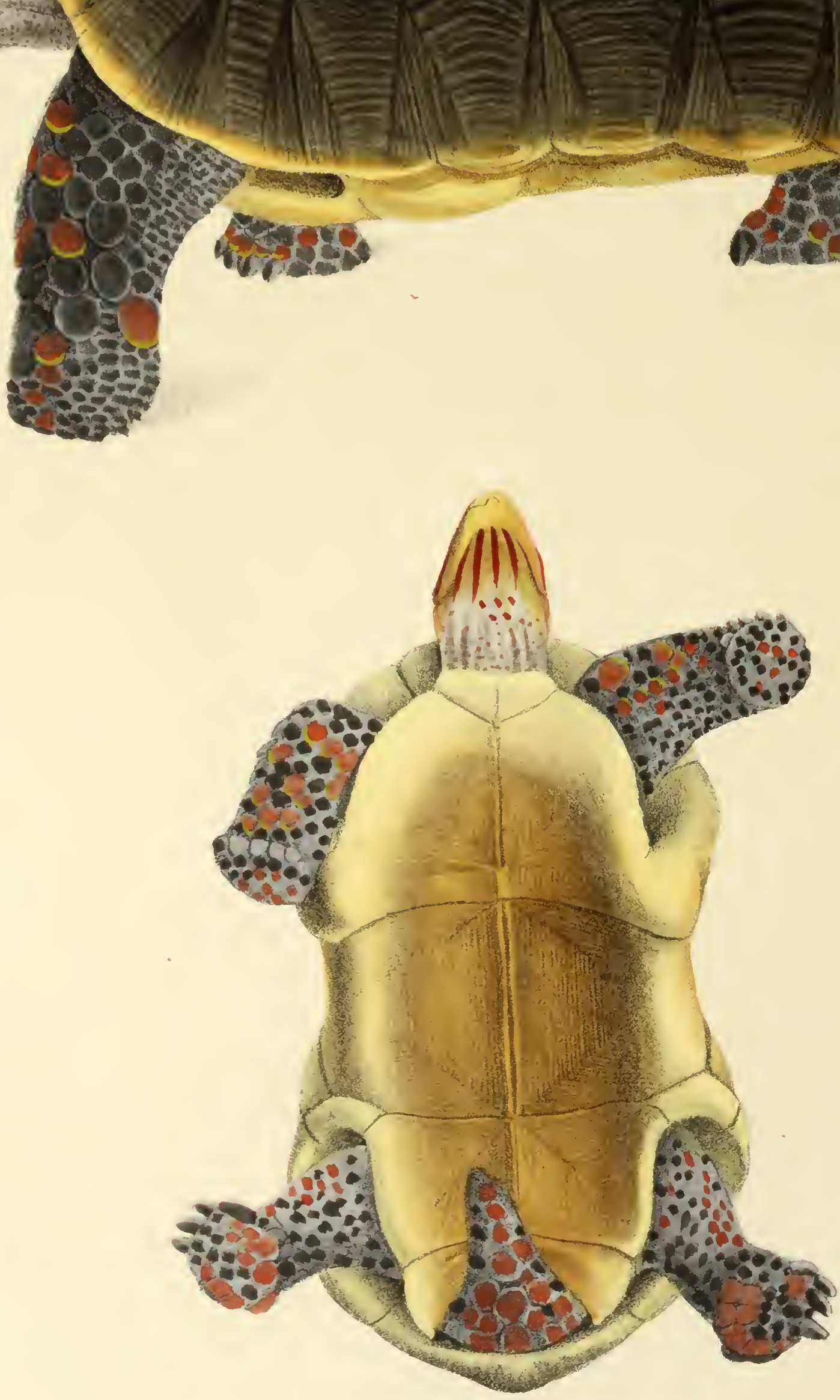

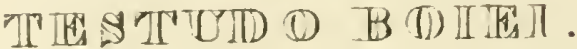

'Tak:XIII 


\section{T E S T U D O B O I E I.}

\section{$\begin{array}{llllllllllllllll}\text { B } & \mathrm{O} & \mathrm{I} & \mathrm{E} & \mathrm{S} & \mathrm{S} & \mathrm{C} & \mathrm{H} & \mathrm{I} & \mathrm{L} & \mathrm{D} & \mathrm{K} & \mathrm{R} & \ddot{\mathrm{O}} & \mathrm{T} & \mathrm{E}\end{array}$}

\section{T A B. XIII.}

Character essentialis. Testa oblonga, ephippii-formi, postice dilatata, rotundata, recto-, antice plano-descendente, subangustata et marginibus subexpansa; scutellis disci sulcatis piceo-nigris, areolis flavidis obsolete gibbis, laevibus, bracteis marginalibus, piceo-nigris, margine infimo flavido; sterno impressissimo; cauda pedibusque squamis rubris, capite scutellis aurantïs flavisque variegatis. (Mas adult.)

Synonyma. Testudo terrestris a mericana Kil. Stobaeus in Act. Litt. et scient. suec. 1730. p. 59. (excl. syn. Marcgr. Test. geometr. ab auctoribus inter synonyma Testudin is tabulatae fialso recepta;) descriptio animalis junioris. Testudo .... Gmel. Syst. Nat. p. 1045. 1. 33.

\section{E S C R I P T I O. ${ }^{*}$ )}

Corporis forma universalis. Caput subdistinctum oblongo-ovatum, angulosum, rostro subproducto, alto, compresso, obtuso-acuminato, pileo depresso, plano, temporibus subrotundatis; 11 ares angustae, longulae, subobliquo-horizontales; maxilla superior inferiorem pyxidum instar includens tomiis rectis, integris, acutis, dertro elongato antice in medio semilunulari-emarginato; oculi modice magni subobliqui; aures squama majuscula, ovata, molli, frons scuto magno, pone acute lanceolato, antice rectotruncato, obsolete gibbo, rostrum supra scutis duobus magnis, regio supra orbitas ac tempora scutellis polygonis, impressiusculis, occipitis margo posticus scutellis subquadratis parvis, planis, regio circa oris angulum scutellis irregularibus, rugulosis, impressis tecta; scutum oblongum supra utramque aurem simileque juxta utrumque latus partis posticae scuti frontalis; collum modice longum, crassiusculum, teretiusculum, cute sublaxa, circa occiput et juxta latera subplicata, tota verrucis minimis, depressis, in mento latioribus, subscutelliformibus, applanatis tecta. Testa elongata, convexa, in medio supra et ad latera ephippii instar maxime impressa, in parte postica quam antica paullo altior, multo latior, convexior, rotundata, postice maxime rotundata et rectiusculo-descendens, margine ibi introrsum, ad latera extrorsum flexa, parte antica maxime convexa, versus latera planiuscule descendente, margine antico supra collum descendente, sinuata, laterali extimo protracta, paullo ascendente, supra pedes subsinuata; scutella disci tredecim, vertebralia quinque, lateralia quaterna, magna, polygona, lineis numerosis profunde exsculptis, rectis sulcata, areolis angulosis obsolete gibbis, laevissimis, in vertebralibus scutellis in medio, in lateralibus et in ultimo vertebrali supra centrum sitis; scutum vertebrale primum quinquangulare ultimo paullo minus, angulo antico protracto, acuto, circa et supra areolam convexum, secuudum et tertium magnitudine aequalia, sexangularia, illud in medio gibbum, hoc planum, quartum iterum in medio, praesertim supra areolam gibbum, sexangılare, postice angustatum recto-truncatum, quintum ingens, postice maxime dilatatum angulato-arcuatum, toto convexum, areola extra medium sita; scutellum late rale primum circuli quadrantem aecuans margine externo dilatatum, angulato-arcuatum, marginibus internis distincle angulosum, supra areolam arcuatam gibbum, fere sexangulare, lateralium angustissimum, impressun, areola plana vix prominente; tertium secumlo majus, applanato-convexum, quinquangulare, quartum subtus subdilatatum, subobliquum, quadrangulare areola subprominente; bracteac marginales viginti-tres, absque areolis, quarta, quinta et sexta exceptis, margine infimo areola subquadrangulari parva, obsolete aut distincte impresso-punctulata, ac insuper infra areolam rngis profunde impressis, angulosis, transversis auctis, omnes lineis versus bracteae apicem in-

*) Maris adulti.

Waglen, AMpuib. II. 
feriorem semsim brevioribus, mumerosis, transversis, in marginibus lateralibus lineis Inngitudinalibus, in suturae bractermm binarum apies supremo couveregentibus, triangulum inter bractearmm biuarum suturam forman-

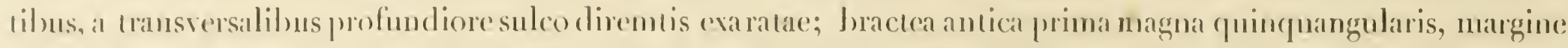
extimo descendente, secunda quitlangularis, margine extimo smbascendente postice angustatia, tertia secundâ

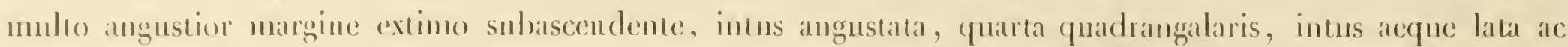

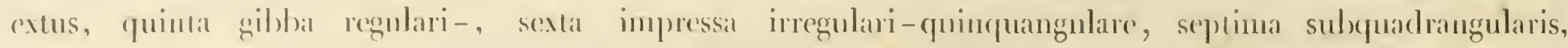
plaul, intus paullo dilatata, octava angusta subquinguangularis, parte suprema plana, inferine intumiclat, nona qualrangularis, parte suprema impressa, inferiore ascendente, extima antica introrsum, postica

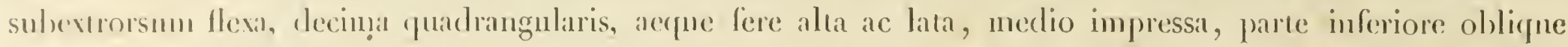

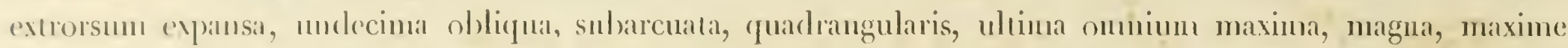
conrexa, quadrangularis, margine infimo introrsun flexa; sternum cum thorace intume conjunctum ejusdem fere longitudine, (puan maxine excavatum, antice et postice aequali latitudine, antice ascendens, apice cxlimo angnstato recto-truncatum, integrum, narginibus planis, postice descendens profundissime arcuatu-cmarginatun, margine distincto, proninente, utroque entarginationis apice acuto, bracteac marginalis ultmae marginem lere atlingente; scutella duodeciun, exclusis duobus parvis pone antiperles et scutello quin(fuangulo ante scelides, supra apices juncturac sterni sitis; scutellorum, praesertim intermediorum suturae rugosac, funminular, scutella ipsa lineis profundis anguloso-dispositis exarata, marginilous extimis integra, laevia, dun antica parva, triangularia, sequentia dun magna, quadrangalaria, margine antico interno obliquo, sequentia (luo angustissina, evtus supra juncturam latiora, intumida ac ibi lievia, intus rugis transversis aucta, lacenia transversal quasi scutcllata intermptis, scutum octavum nonumque onmium maxima, magua, quadrangularia, decimun undecinumuque quadrangularia antecedentibus dimidio fere minora, ultima duo angustissima, trigona: margo junctura sterni cum thorace rotundatus; pedes toto sfultunosi, antici compresso-angulosi mugnil)us (nuinque, postici teretes unguibus quatuor, ungue primo oblique subincumbente; u squa nu a antiperlum magnae, scutelliformes, planae, laeves, quarum majores margine antico patentes, scelidum parvae, quadratac, onnes appressac, planac, pronimulae, circa basin plantac magnae, polygonae, scutetlifornes, plinae, posticarmu una maxima margine infino libero, insistente, plantae majusculae, planac, polygnae, palmac ejusclenu forntae attamen minores; cauda conica longula, crassa, depressa, apjece rotunda, scpuamis supra magnis minoribusque applanatis, irregularibus obtecta.

Color. Pedes et cauda nigra; squamae antibrachii anticae majores cimnabarino-rubrac margine flavo, posticac duo magnac ejusdem picturac; squamac in rhizonychii basi flavac, superius rubro-marginatae, palmae plantae ac superioris lateris caudae testaceo-rubrae, intermixtis squamis nigris; collum cincreo-rufescens (in anmale jurenili cincreum) immaculatum; mentum punctulis nonnullis et vittis quatuor rubris; caput fusconigricans sculis suprat nasum et verticen aurantiacis; scutum supra aures oblongun aurantio-rubrum; genae inferius ac occiput rubro-punctata; irides flavidae; palpebrarum margo inferior ruber; testa picea, areolis scutellormm disci flavidis; ejus nargo inferior ac sternum flavida, hoc fusco-adumbratum, et intus ante temora rulso-cincinn; rhamphotheca corneo colore; ungues nigricantes apjice albidi.

Proportio corporis partium e mensura. Longitudo a rostri apice usque ad caudae finem (juxa testam) 20\%", caudac solius 3\%", capitis 2\%", colli 20 , brachii $2 \% "$, antibrachii 3\%", fenoris et Libiar 2", lhoracis 13\%", sterni 10\%2", ambitus occipitis collirue 5" 2", testac inmediate pone antipedes $20 "$, in medio 18\%", immediate ante antipedes $21 "$; altitudo testae 5", in medio $4 \% 2$; latitudo sterni immediate pone antipedes 4\%", ante scelides 5"; oculi distant a naribus 4", a rostri appice 10".

Inimal junius. Thorax arluli, testa supra et ad latera minus impressa, pagina superiore fere recta, marginibus mimus expansa; color adulti, si sternum excipis flarum, corneo-nulbilanm, in unedio macula oblongo-hexagona signatum; mentum absque lineis quatuor lougitulinalibus rubris; squamae: rubrae antiperlum vis flavo-marginatac; vertex macula $T$ - formi, pone macula utrincpue prarva rotunda, concolore ancta, flava notatus.

Habitatio el vitae ratio. Habitat....?

OBSEMVATIOXES. Specinen depiclum xirum possedil Monachii comes a Bulller idque ad describendum mecum commmnicarit. In animale effarclo, nunc in musen nostro obvio, pedum squamae rubrae mox

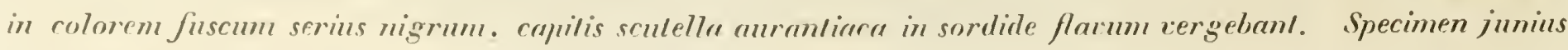
possidel vir supra dichs. 
MC7 LIS ARY
RVARO UNIVERSITY

CAMBRIDGE. MA USA 


$$
8
$$




\title{
B I P E S P A L L A S I I opp.
}

\author{
P A L L A S I S C H E P A N Z E R S C H L E I C H E.
}

$T_{\text {A }}$ в. XIV.

Character essentialis. Corpore toto rufescenti-fusco aut sordide stramineo, unicolore; squamis trunci et caudae annulari-dispositis subosseis.

Synonyia. Lacerta a poda Pall. It. 3. p. 702. Nor. Comm. Petropol. 19. p. 435. t. 9. Lacerta a pus Griel. Syst. Nat. T. 1. P. 3. p. 1079. n. 77. Cha ma esaura a pus Schneid. Hist. Amph. II. p. 212. Bipes Scheltopusik Bonnat. Encycl. meth. T. 1. P. II. p. 68. t. 12. 1. 7. Sheltopusik didactylus Latr. Rept. 2. p. 273. Seps Sheltopusik Daud. Rept. 4. p. 351 . Bipes Pallasii Opp. Rept. p. 43. Pseudopus serpentinus Merr. Syst. Ampl. p. 78. (Pseudopus Oppelii Frtzing. Classific. der Rept. S. 50.)

\section{E S C R I P T I O.}

Corporis forma universalis. Caput subdistinctum tetragono-ovatum, aeque fere altum ac latum, rostro longo, compresso, acutiusculo, supra a fronte rersus apicem subtiliter devexo; regio ophthalmica impressiuscula; maxilla quanı mandibula vix longior, hanc non includens; lamina supraorbitalis ossea; asserculum obtusiusculo-acutum; nares subapicales infra finem canthi rostralis distincti, sed subrotundati intra scutella nonnulla irregularia sitae, subrotundae, simplices, extrorsum patulac; oculi naribus paullo propiores quam auribus, horizontales, pupilla circulari; palpebra superior ac inferior completa; a ures distinctae multum ab oris angulo distantes, longitudinali-rimiformes, simplices, membrana tympani profunde sita; lingua pone multum dilatata, antice oblonga, apicis rotundati medio emarginato-furculata, emissilis, supra papillis mollibus obtecta; dentes primores ossis intermaxillaris octo, approximati, lomogenei, robusti, cylindracei, erecti, recti, corona simplici rotundata, extinus utrinque a reliquis subamotus, maxillares heterogenei, erecti, recti, validi utrinque tredecim, simplices, octo anteriores subdiscreti, reliqui approximati, primus, secundus et tertius primorum magnitudine et forma, quartus, quintus, sextus, septimus ejusdem formae sed sensinı majores, octavus et nonus omnium maximi, validissimi, corona globulosa, decimus, undecimus et duodecimus homogenei et praecedentium magnitudine et forma sed dimidio minores, tredecimus minimus corona orbiculari, mandibulares maxillaribus quoad situm similes, utrinque tredecim, heterogenei, anteriores sex lomogenei obtuso-conici, multum discreti, septimus et octarus homogenei his paullo majores, corona rotundata, nonus onmium maximus, maxillari nono paullo minor, corona oblongo-globulosa, sequentes tres magnitudine et forma octavi, ultimus his paullo minor ejusdem formae; dentes palatini distincti, numerosissimi, conici, utrinque per series duas irregnlares dispositi; truncus longissimus, cylindraceo-compressiusculus, cum collo undique aequali crassitie, utrinque ad latera a brachii regione usque ad scelides plicato-unisulcatus; collum compressiusculum, simplex; pedes antici externi nulli, posticorum solum tibia abbreviata, metatarso incompleto, adactylo interdum subbipartito aucta, supra sulci lateralis finem inserta, brevissima, compresso-subulata; cauda longissima tertia parte corpore longior, tereti-polygona, sensim in acumen strictum desinens, integra. Pholidosis dura, caput supra scutis multis, maxime ossibus appressis tectum, ad latera fere squamosum; scutum verticale omnium maximum, ovatum, postice rectiusculo-truncatum; sfuamae trunci caudaeque osseae (attamen epidermide subtili obtectae), postpositae, plus minusve rhombeae, in trunci et caudae parte inferiore marginis postici medio emarginatae, trunci ceterae laeves, caudae carinatae, unde ibi lineas longitudinales prominulas efficientes, in trunci et caudac parte superiore

Wagten, АмPHib. II. 
arepun moto lincari-carinatare, practerea radiatae, sed margine postico non emarginatac, homogeneac; ani

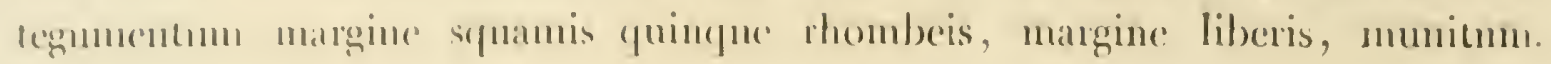

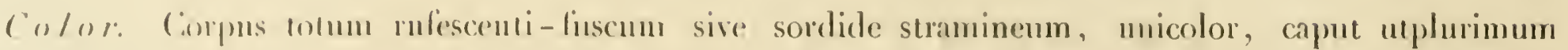
relippis corporis partibus dilutins; irides in fundo griseo punctis creberrimis aureis, pupilla magna, rotundis.

Proporlio rorporis parlium " mensura. Longitudo a rostri apice usque ad caudae finem

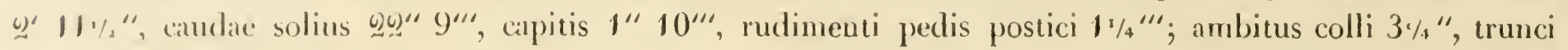
prope aи111 2 " 11 ".

Ilabilalio l, vilae ralio. Habitat in convallibus herbidis deserti Sibiriac australis Naryn et al fluvios Sarpan, Kumam, Terehum ac in Dalmatiae vallis fruticosis ac saxosis aquis confinilus, Inguis fragilis in modum infa frutices saxosque degens; animal mitissimum, Angui quoad corporis motus, ac virendi rationem reliquam simillimum.

OBSERVATIONES. Bipes Pallasii olim rarissimus in Museis zoologicis adest nunc in mullis collectionibus tam publicis, tam prixatis; duos vivos observare, aliorumque fabricam corporis internam examinare mihi licuit. Specimen al OPPExın 1. c. descriptum dalmalicum, alio, quod possideo, dalmatico in ommi puncto simile. si excipis pedes in hoc integerrimos, mecum uxor cl. viri communicavit. In exemplo illo pedes in apice subbiparlitos sed non vere digilatos, ac in eorum margine postico sryamulas nonnullas punclatas, sed non porosas, offendi. Similem struclurain pedum vidil PAL.LAS. Non dubium unde videlur quin sunumi viri Lacerta apoda huc referenda sit. Al eos admonilos volo, quibus specimina sibirica intuendi facultas, "t in hanc rem accuratius inquirant. - Specimina in spiriln vini asser. vala parun aut cix pallescunt. 



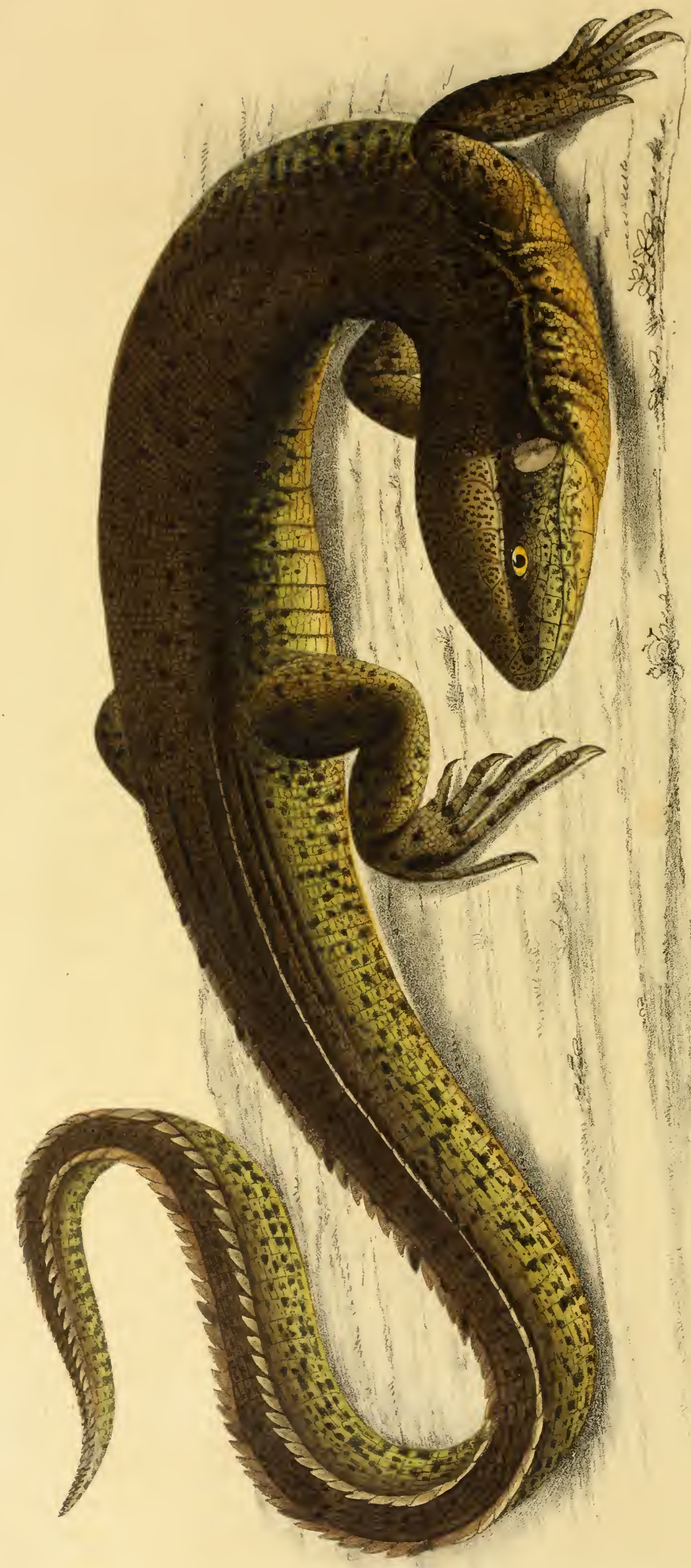

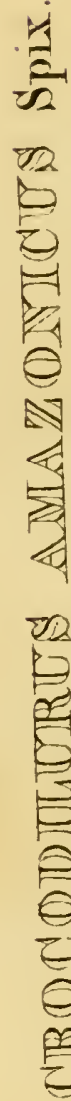




\title{
CROCODILURUS AMAZONICUS
}

Spix.

\author{
E I D E C II S E N A R T G E R C R O G O D I L S G H W E I F.
}

\section{T A. B. XV.}

Characteressentialis. Corpore supra fusco obsolete nigro-maculato, subtus flavo, ad latera virente maculis parvis, in cauda subquadratis, nigris, variegato; collo subtus vitellino; digitis nigro-annulatis. Anim. adult.

Synonyma. Tupinambis lacertinus Daud. Rept. II. p. 85. (Descriptio animalis junioris optima.) Le Lézardet Cur. R. an. II. p. 26. (citata Lacerta bicarinata LinNaEI vitanda) Crocodilurus amazonicus Sprx Lac. bras. t. 21. (Anim. adult.; fig. non satis a c c u ra ta.)

\section{E S C R I P T I O.}

Corporis forma universalis. Caput tetragono-pyramidale, longulum, occipite aeque alto ac lato, indistincto, supra omnino planum, a fronte versus rostri longi apicem compressum, acutiusculum, parum declive; maxilla mandibulae longitudine; lamina supraorbitalis cutanea, asserculo vero nullo; oris rictus vix ultra oculos extensus; labia non prominula; canthus rostralis distincurs, acutus; nares in canthi rostralis apice in foveola profunda trigona, sitae, mediocres, retrorsm patulae, simplices; oculi auribus paullo propiores quam naribus, pupilla circulari; palpehrae superioris rudimentum; t ym p a n um altissime situm, admodum planum, magnum, rotundum;, mandibulae latera postice musculoso-intumida; lingua musculosa, planiuscula, emissilis, apice furcata; dentes alternantes, primores ossi intermaxillari inserti decem, aequales, erecti, graciliores, conici, simplices, subdiscreti, maxillares heterogenei, utrinque quindecim, primus corum a primoribus diastemate angusto direnutus, parsus, primormm lougibuline, simplex, subcurvatus, secmolus, terius quartusque forma et magniumline aequales, omnimu fere longissini, simplices, tretes, conico-acmminati, sulurelinati; punintus et sextus parvi, pracedentibus dimiclio minores, obsolete tribhi, septimus reliquis sequentibus forma et fere magnituline similes, secundi longitudine et eodem validior, erectus, corona acuta distincte triloba, mandibulares septendecim, prinores discreti, ommes, exceptis sex primorihus, magnituline et forma silsi similibus, mreribus, simplicilns, conico-acmminatis, subreclinatis, superiormm posticormu magnituline, tres corum anteriores olsolcte-, reliqui distincte triblsi; dentes palatini nulli; truncus molice longus, teres, pectore applanato; cutis ad latera impressionihus V-formilous, cohaerentibus aucta; collum longulum, trunci fere crassitic, lateraliter rugoso-, subtus distincte bi-plicatum; cauda corpore toto tertia parte longior, alta, admodunı compressa, culmine applanata, unde ibidem acute bi-angulosa, suhtus rotmelata, arl latera plana, sensim acuminata, simplex; pedes modice longi, pentadactyli, crassi, postici teretes anticis compressinsculis dimidio validiores; digiti palmae teretes, brevinsculi, robusti, toto liberi, tertins a quartus longitudine aequales, plantae graciliores, liberi, maxime inaequales, quartus longissimus tiliac Iongitndine; pori femorales distincti; ungues validi, hreres, arcuati, compressi, acutissini.

Pholzlosis. Caput supra et ad latera scutis lacvibus polygonis, majusculis, numerosis, gula et collum suhtus squamis scutelli-formilons quadratis, planis, abdomen totum scutellis regularibus, quadrangulis, planis, margine postico patentil)us per series transversas digestis, anus supra scutellis irregularibus tecta; trunci superioris squamulac numerosissimae, oblongo-quadratae, compressae, unde subelevatae, medii homogeneac, lateralis ejusdem formae sed majores, cervis granulosae, omnes fasciatim dispositae, caudae

Wagter, AMPHIS. II. 


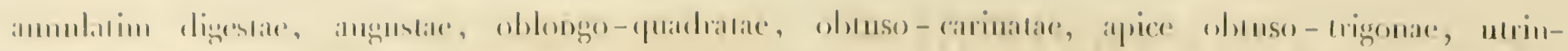

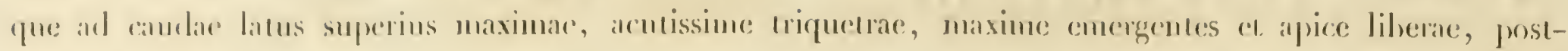

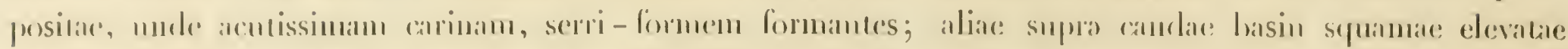

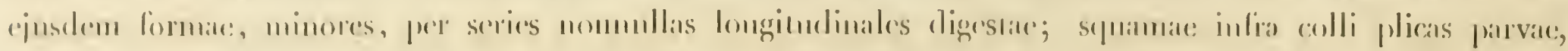

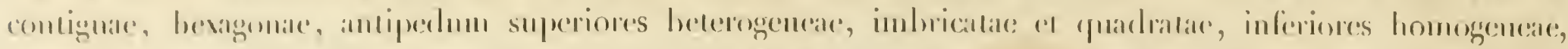

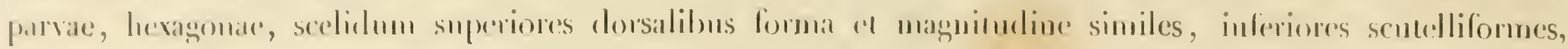

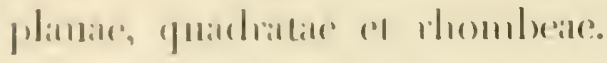

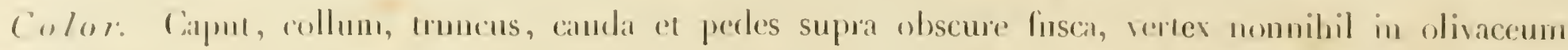
vergens maculis pusillis mumerosis, subobsoleris, nigricantibus, dorsum et pedes supra talibus maculis sed majorihus variegata; caput, collum et truncus subtus flava, immaculata, collum obscurius, fere vitellinum;

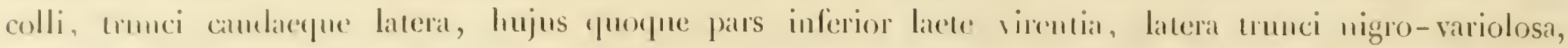

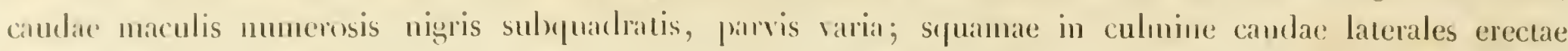
pallide luscar intermixtis macnlis nigricantibus; digiti nigricanti-annulati; irides rubro-flavae.

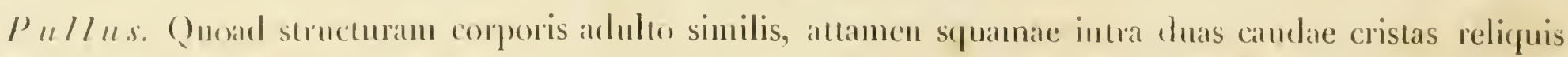
vis majores; capur, collum, truncus et cauda supra pallide fusca; dorsun ocellis dilutioribus obsoletis varegatum; colli, trunci atc camtae latera caesio-nigricantia, ocellis magnis rubicundo-allbis belle variegata; camla tota suhtus catesio-nigricans, unicolor; squamac utriusque cristae culminalis el luscescenti-albae et toto nigrae: (aput, collum et truncus subtus allsa, maculis irregulariter dispositis caesio-nigris, irregulariLus; antipertes scelielespue rubicundo-albidi nigro-reticulati, antipeeles subtus magis maculati; digiti in eonlem fundo nigro-annulati; ungues dilute fusci; scuta marginalia labii inferioris allida, supra sutulas nigro-llarginta; masilla vertici concolor; cauda subtus rotundatil sed versus finem fere quadricartnala. (I) nesciplin especimine in spirilu viui asservalo.)

S) nony min: Cirocodilurus ocellatus Spr. 1. c. 1. 22. r. 1. (lig. lona.)

Proporlin corporis parliume mensura. Longiturlo a rostri apice usque ad caudae finem 2-", caudae solius 178/2", capitis $2^{\prime \prime} 2^{\prime \prime \prime}$, ejusdem altitudo supra aures fere 1\%", totidem latitucho supra aures.

Ilabilatio elvitae ralio. Habitat in Guiana ac in Brasilia rersus flumen Amazonum.

O BSERV ITIONES. Specimina dun hajus speciei adsuml in Museo Monacensi; jumins exemplum a cl. 1) AVIIX descriptum possidel Museum Parisiense; hoc, quocum specimina nostra prorsns cameeniunt ipse ridi: Crocodilnri amazonici diversilatem igilur conlendil frustra FHTZJNGER (Isis 1827. p. 746.) LANAEI Lacerla bicarinala, (CEPEnI Dragonne, SchNeIDER Lacerla Dracaena Denkschriflen der Mï̈ch. Akad. der Wr. 1821. Bd. 8. S. 1.57. 1.8.), tuam ad Tnpinambem lacerlinum referplamil DAUm, ac CUrren, differl lolo coelo ac formal semus nostrum "Thoriclis."

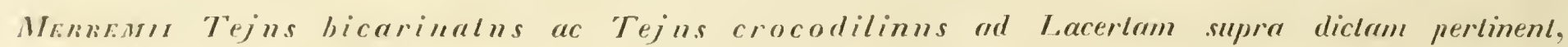
red ambornum symonymia, maxime confusa, spectal el ad hanc speciem el ad Tupinambem lacerli-

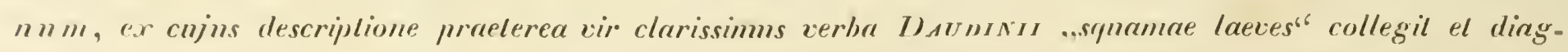
nosi sni Teji licarinali insernit. - Colores virides specimimum effarclormm ant in spiritn vimi adservalormm mox ad sordide flavmm inclinam, reliqui restant. 


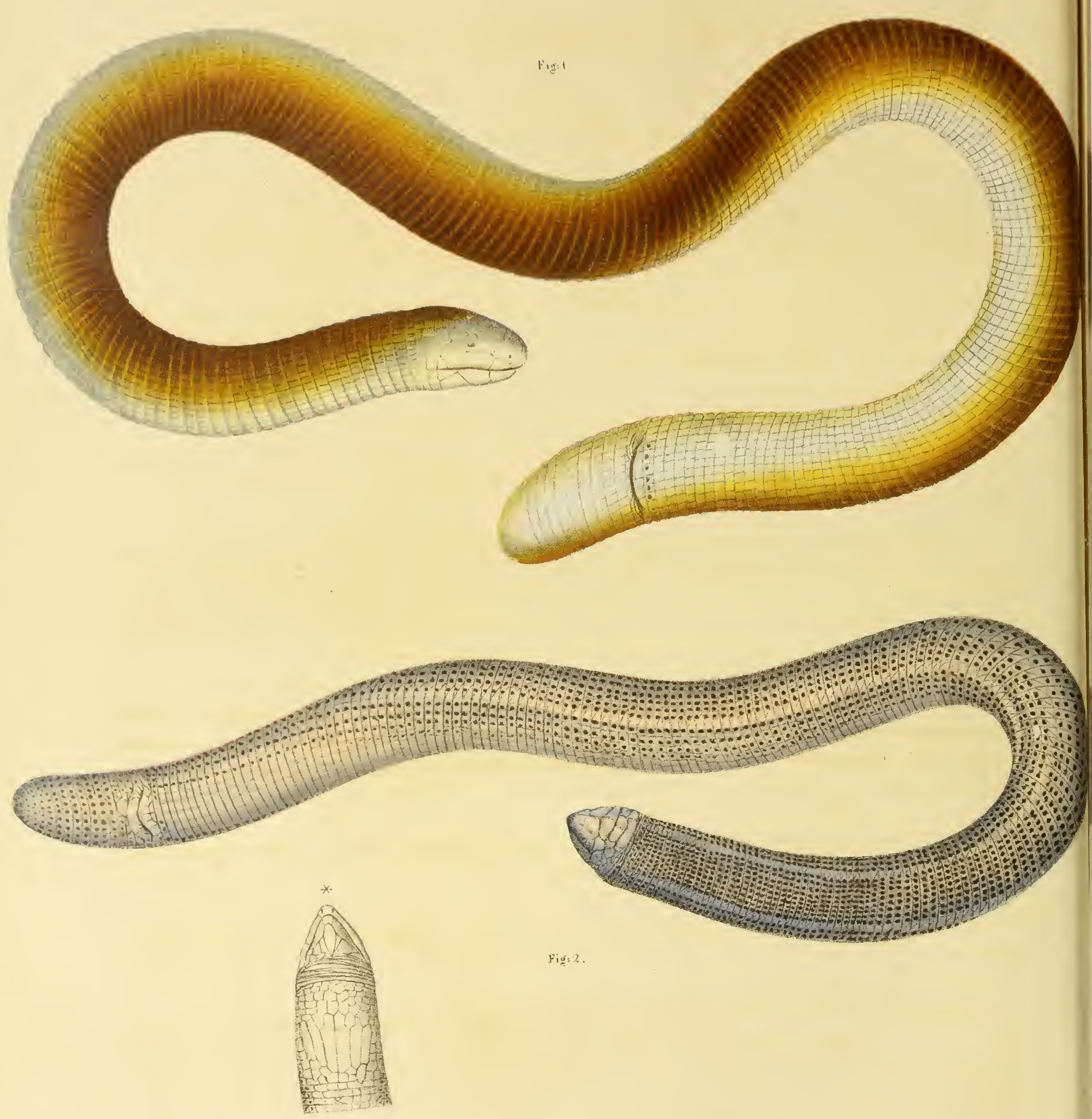

Fig: 2.

ATIPHISBAENA FHATESCENS。Neur. Fig: 1.

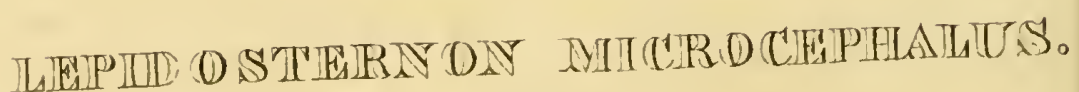
Fig:2.

Tab:XV1 


\title{
AMPHISBAENA FLAVESCENS Neur.
}

\author{
G E L B L I G H E D O P P E L S G H L E I C H E.
}

T А в. XVl. F I G. 1.

Character essentialis. Nitide flavo-fusca, lateribus trunci dilute flavis, abdomme coernlescenti-albo; cupite albido; poris supra unum sex; trunci annulis 292, cuutue 14-15.

Synonmia. A mphisbaena pachyura Worf Abbild. und Bescheeil. merhwärdiger naturgeschichtlicher Gegenstände II. p. 61. t. 17. (Icon e specimine decol.) Amphishaena flavescens Neuw. Beytr. zur Naturg. Bras. 1. p. 50\% (Descriptio opt.) ID. Abbild. zur Naturg. Bras. (Fig. bona.)

\section{E S G R I P T I O.}

Corporis forma universalis. Caput ovatum, trunco fere angustius, laeve; maxillae apex acutiusculus largiuscule supra mandibulae apicem extensus; oris rictus rectus, modice magnus; nares ad rostri latera fere in ejus fine, parrae, formes; oculi capitis cute commmi tecti, vis in modum puncti nigri pellucentes; lingua ac dentes congenerum; truncus elongatus, crassus, cylindricus, undique aequali ambitit; cauda brevis trunci crassitic apice obtusissima, rotumdata.

Pholidosis. Rostrum totum ac frons impressionibus cutis scuti-formibus polygonis; truncus impressionibus annularibus 222, satis profundis; annuli ipsi lineolis longitudinalibus crebris exarati; cauda aequo modo annulata ac lineolata, impressionibus anuularibus $14-16$, apice extimo integra; pori ante anmm ses distincti.

Color. Corporis partes superiores omnes eleganter flavi-fuscae, nitidae, laterales dilute flavae, inferiores glauco-albae, hyalino-nitentes; rostrum albidum.

Proportio corporis partium e mens ur a. Longitudo 19" 3", candae 1" g", capitis $1 \%$

Habitatio et vitae ratio. Habitat, utplurimum sub terra degens, in Brasiliae provincia Bahia, ar in regionibus prope flumina Belmonte et Vucuri sitis, in sylvis inter Bromelias;

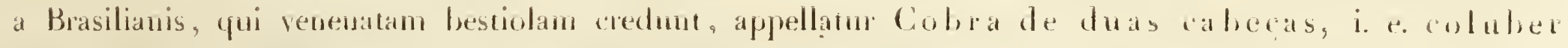
biceps.

O B SER VAT IO. Specimen delineatum purae conservationis adest in mea collectione: picturam ex principis seren. NEUNIED icone ac descriptione hansi. Specimen in spirim vimi conditum apparet in corporis partibus superioribus dilute fuscum. in partibus inferioribus sordide allmm. Amplisbaena alba, a Spixio in Brasilia non obsemata. sed freqnens in Surinamo obviu. differt a hac specie pictura ac poromm ani mmero, quomm in Amplistaena alba constanter novem conspicinitur. 


\title{
LEPIIOSTERNON MICROCEPHALUS.
}

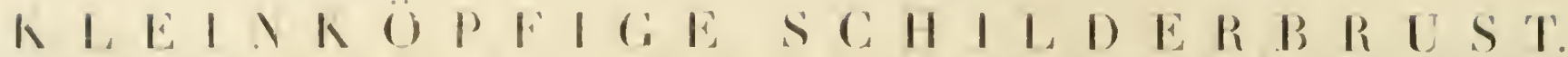

$$
\text { T I i. XVI. Fir. }
$$

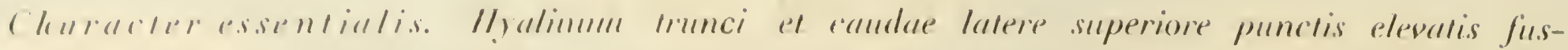

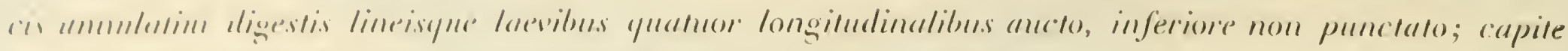

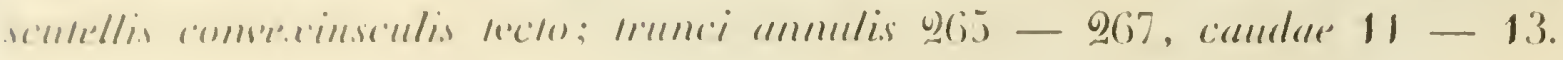

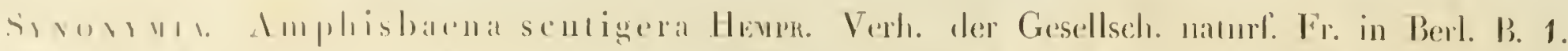

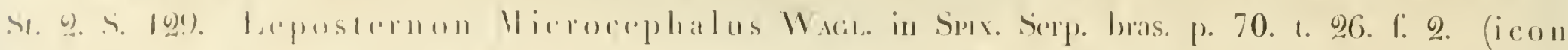

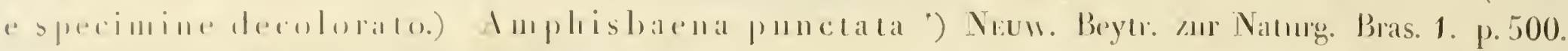

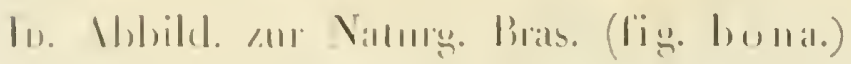

\section{1) ES S R I P T I O.}

C'orporis forma universalis. Catput parvum trunco angustins, brevi-conicum, postice sube-

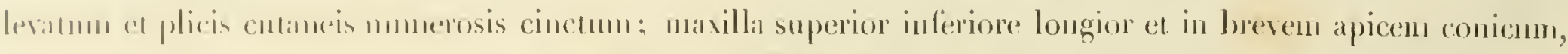

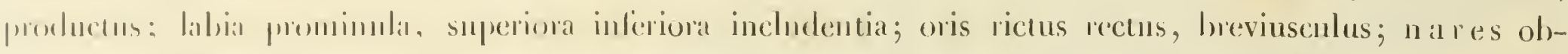

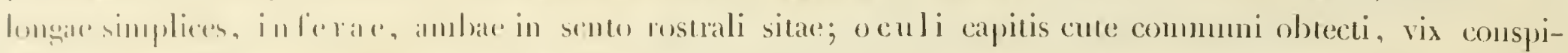

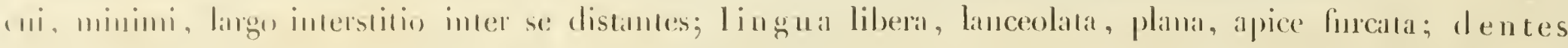

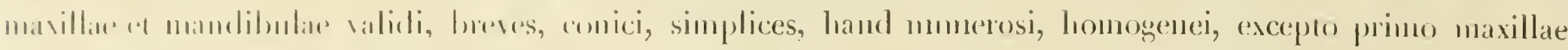

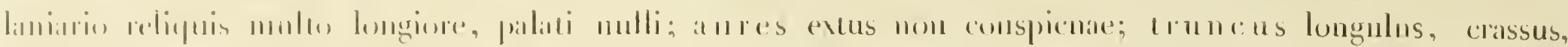

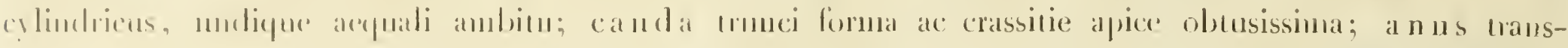
resils, lillus.

Pholidosis. Scuta in capitis parte superiore subconvexa obsolete angulosa; scutum verticale subrotundım, lere septangulum; scuta ocularia subquadrangula; scutum marginale labii inferioris utrinque unicun, maximun, subtriangulare; sculum mentale unicum, longiusculım, subcordatum, obsolete angulosum; scu-

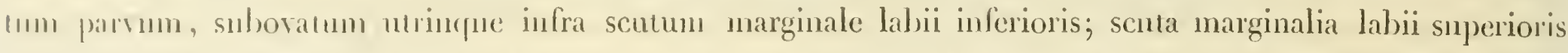

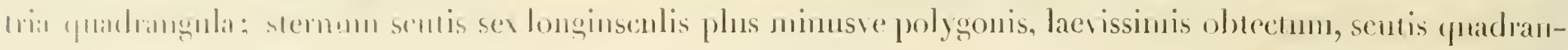
sulis mommllis partis inlia " jula illat almevis; truncus candaque fola annulis impressis mumerosissinis,

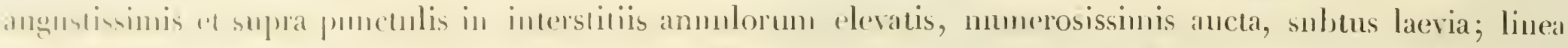

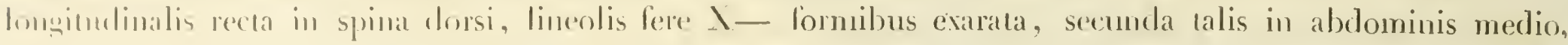

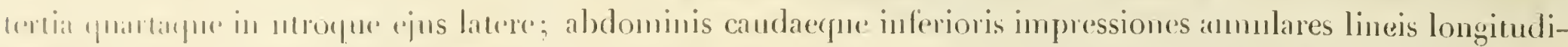

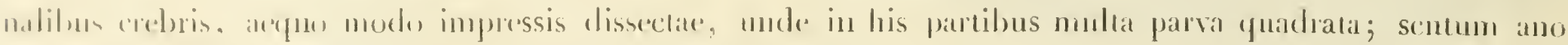

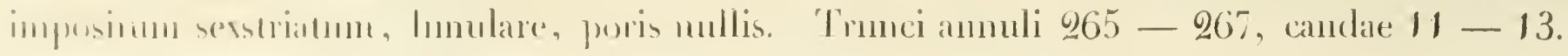

lolor. Corpus pallidissime violaceum sive lilacinum, fere hyalimm; colli color magis coerulescens,

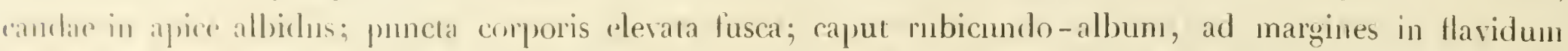

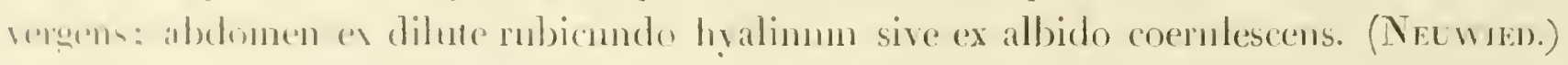

Propurlio corporis parlinme mensura. Congitudo at rostri apice usque ad candae finem

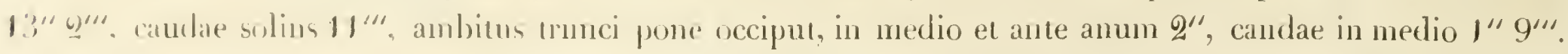

/l"bil"lin"l viloe rolio. Habitat in Brasilia a Rio de Janeironsque arl flumenEspirito

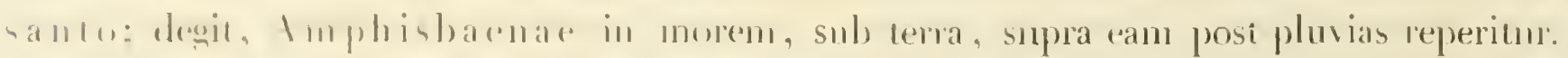

UBSERVATHOLS. Speciem hane habel Musenm Herolinense. Lugdunense ar Monacense: specimina necata mose in rordide allumm rerguml. 



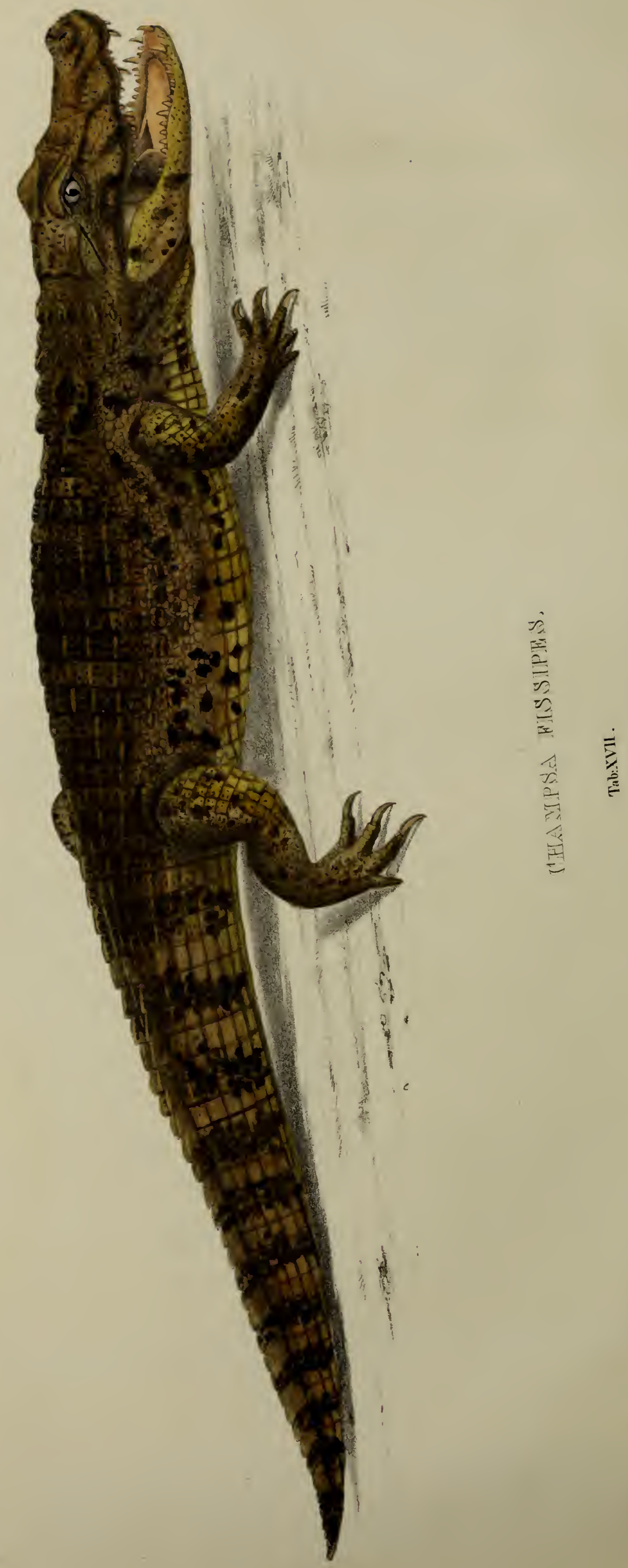




\title{
CHAMPSA FISSIPES.
}

\author{
S P A L T F Ü S S I G E R A L L I G A T O R .
}

\section{T \ $_{\text {в. }}$ XVII.}

Character essentialis. Plantae digitis liberis; porca inter canthos anteriores oculorum; corpore supra olivaceo, nigro-vario, subtus flavo, ad latera fiusco-maculato.

Synonyma. Caiman fissipes Spix Lac. Bras. p. 4. t. 3. (fig. bona.)

\section{E S C R I P T I O.}

Corpors forma universalis. Caput depresso-ovatum, latum, obtusum, breve Champsae Lucii, diversum ab hujus capite porca inter canthos anteriores oculorum, admodum elevata, oblique utrinsecus versus maxillae tomia supra dentem nonum excurrens; rostrum ac maxillae latera foveolis rugosis; orbitarum margo superior elevatus; interstitium inter utrumque angustum, versus porcam inter oculorum canthos anteriores situm sensim latius, postice in vertice planiore sed ter impresso et canaliculatorugoso late excurrens; supercilia cutanea antice lamina ossea trigona suffulta, pone cutaneo-acuminata; nares, oculi, ac aures congenerum; tomia distincte undulata; dentes alternantes, mandibulares omnes introrsum, maxillae omnes extrorsum versi, maxillae triginta sex, totidem mandibulae, ceterum ut in congeneribus formati; collum et truncus congenerum; cervix fasciis scutorum quinque; scuta fasciae primae octo angustissima, circuli segmentum breve aemulantia, latis intervallis inter se distantia; duo intermedia eorum omnium minima, reliqua magnitudine fere aequalia; scuta fasciae secundae tertiaeque in modum nunc descriptorum formata, iisdem minora, fasciae secundae quatuor, latissimis intervallis inter se distantia, duo intermedia eorum minima, fasciae tertiae octo, in modum praecedentium posita, duo intermedia ommium maxima; scuta fasciae quartae quatuor, omnium cervicis maxima, adnodum magna, praesertim duo intermedia inter se in basi lata connexa, planiuscula, quadrata, in nıedio carina valida elevata, rugoso-impressa; scuta fasciae quintae duo, intermediis duobus fasciae praecedentis forma universali, situ magnitudineque similia, carina excepta obliquiore; scuta praecedentium fasciarum laevia; dorsum fasciis scutorum octodecim; prima earum e scutis duobus, secunda ad septemdecimam a scutis sex distinctis, fascia septemdecima et octodecima e scutis quatuor compositae; scuta fasciae secundae lateralia utrinsecus duo plus minusve obsoleta; cauda scutorum fasciis triginta septem, ad latera laevibus, in parte superiore a dorsi fascia ultima usque ad fasciam quindecimam $6-7$, penultima duo \%, antecedentia quatuor 3, carinis, reliquis superius in cristam compressam, simplicem, altam desinentibus; truncus ad latera scutellis oratis, distincte carinatis, per seriem subarcuatanı digestis, infra dorsi fasciam quintam incipientibus, infra tredecimam desinentibus; reliqua trunci superioris, colli ac pedum scutis irregularibus rhombeisque, planis, plus minusve magnis tecta; scutella menti, partis inferioris colli, trunci caudaeque quadrata, laevia, palmae plantaeque subtus squámoso-granulosae; pedes congenerum exceptis digitis plantae toto fissis; digitus extimus eorum cum penultimo basi palama brevissima connexus, muticus, reliqui unguiculati; palmae digiti fissi, tres interiores unguiculati, reliqui mutici.

Color. Olivaceo-virescens; dorsum et cervix nigro-conspersa, illud ad latera maculis nigricantibus variegatum; pedes ex olivaceo-viridi et nigro variegati; cauda ex olivaceo-viridi et ex nigro fasciatovariegata; mentum virescens; collum, truncus et cauda subtus flava, immaculata, abdominis scutis ulti- 
mis exeeptis matula magna irregnlari fusca notatis; capur olivaceo-virescens lituris nigricantibus subtilibus fmuctispue concoloribus, mandibulae latera lasciis obsoletis nigricantibus variegata.

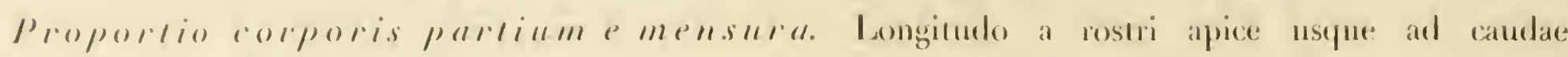

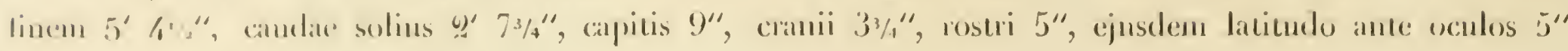

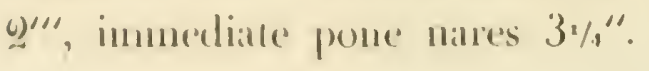

Il"billlio elvill" ralio. Habitat in Brasilia, in terris flumini Amazonum confinibus.

OBSEMVATIONES. Specimina hujus speciei duo adsunt in Mnseo Monacensi, fuorum unum vix bipe. dale, discript" majori, "Sixio allatum in omni puncto simile. 



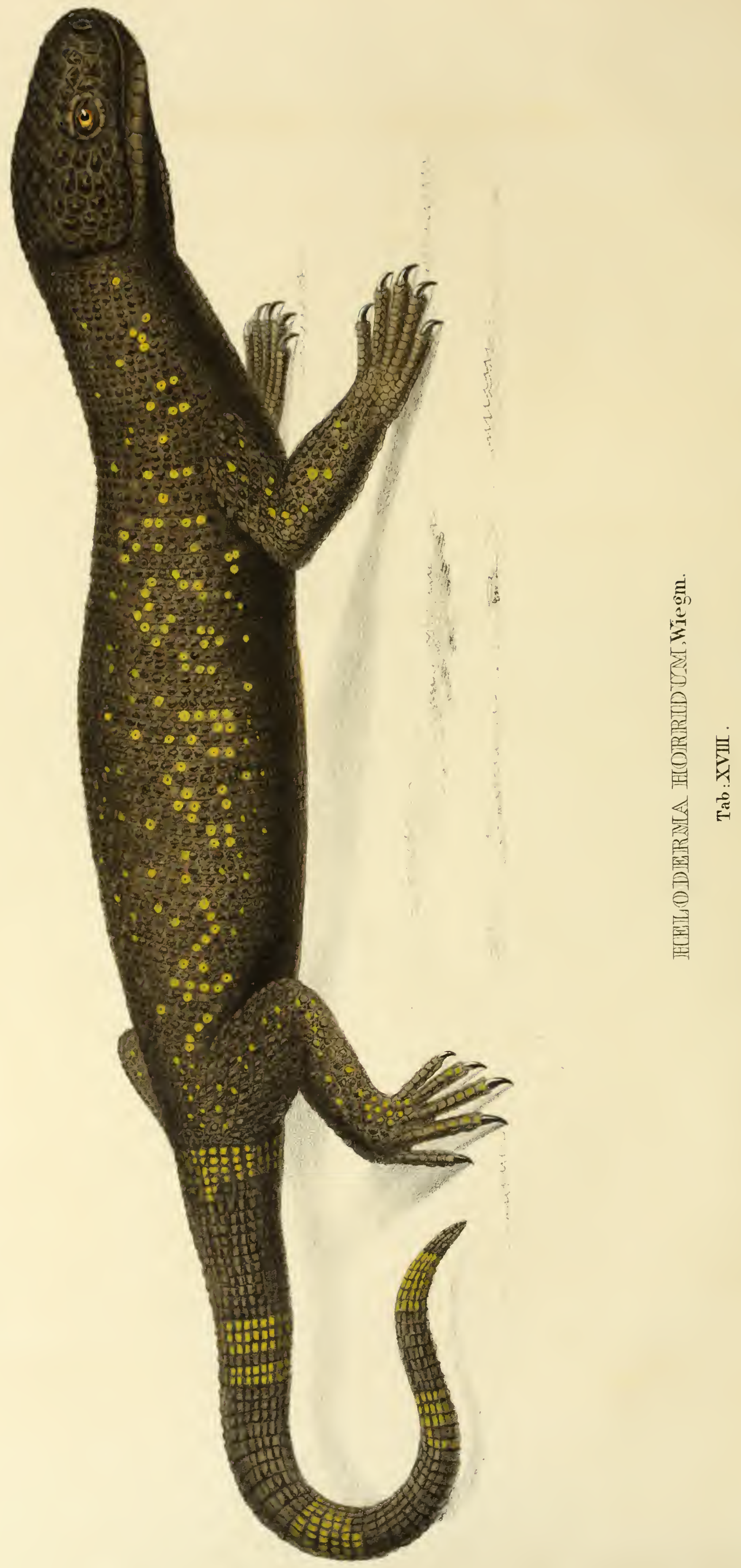




\title{
HELODERMA HORRIDUM
}

Wiegm.

\author{
$\begin{array}{lllllllllllllllll}\mathrm{R} & \mathrm{A} & \mathrm{U} & \mathrm{H} & \mathrm{E} & \mathrm{K} & \mathrm{R} & \mathrm{U} & \mathrm{S} & \mathrm{T} & \mathrm{E} & \mathrm{N} & \mathrm{E} & \mathrm{C} & \mathrm{H} & \mathrm{S} & \mathrm{E} .\end{array}$
}

\section{T A B. XVIII.}

Character essentialis. Terreo-fuscum, squamis durissimis lentiformibus gibbis, supra caput majoribus, subangulosis, nigro-fuscis et aureo-flasis sariegatum; cauda annulis quinque flavis; squamis abdominis oblongo-quadratis, laevilus, planis, fusco-el flavo-variis.

Syonrmia. De Acaltetepon, seu Moneosillo mucronato, quod privatim Tenacuincahura vocant, Lacerto novae Hispaniae, Hervanof. Hist. Nov. Hisp. Cap. Il. p. 315. Heloderma horidum Wieg. Isis 1829. S. 624.

\section{E S C R I P T I O. $\left.{ }^{*}\right)$}

Corporis forma universalis. Caput ovatum, depressum, planum, rostro longo ad latera rotundato, obtusissimo; occiput rostro non altius applanatun, ad latera subintumidum; mandibula maxillae longitudine, hunillima; tomia utriusque rectissima; rictus ultra oculos extensus angulo recto; labia maxillae et mandibulae versus oris angulum proniuula cacterun plana; canthus rostralis of soletus, rotundatus; nares laterales al) oculis largo interstitio distantes, verticali-patulae, magnae, pyriformes, intra squamas tres planas sitae, extrorsum spectantes, simplices; oculi alte siti, subobliqui, nares inter et oris angulum intermedii; palpebrae superioris rudimentum, inferior completa; a ures angustae, perpendiculares, patulae (?); gula inermis, non inflabilis; dentes tomiorum margini adnati, haud numerosi, graciles, conici, acutissini, antico latere intus sulco profundo exarati, homogenei, maxillae primores reliquis breviores, recti, reliqui retrorsum et extrorsum versi, palatini nulli; truncus torosus, longulus, pone antipedes et ante scelides parum attenuatus; collum longulum trunci fere longitudine, crassum, depressiusculum, occipitis latitudine, non plicatum; squa mae capitis, trunci, pedum nec non totius caudae supra et al latera, capitis et colli sultus quoque osseae, maxime gibbae, lentiformes, obtusae, cum tota basi cuti insidentes, rugulosae aut sublaeves, par is interstitiis subgranulosis inter se subdistantes, per series numerosissimas transrersas dispositae, magnitudine formacque omnes aequales, solum supra caput majores et polygonae, partis inferioris trunci caudaeque scutelliformes, planae, laeres, oblongo-quadratae, cuti cum tota hasi affisae, per series transversas digestae; pedes robusti, teretiusculi, modice longi, postici anticis vix validiores, omnes pentadactyli; digiti crassi, breviusculi, tereti-compressiusculi, antipedum omnes liberi, tertius cum quarto, secundus cum quinto aequali fere longitudine, quintus primo paullo longior; scelidum digitus secundus et (puartus cum intermedio cute modice lata usque ad finem phalangis primae connexus, reliqui liberi, tertius cum quarto aequali fere longitudine, extimus vix, sed rersatilis, cum reliquis fere in una eademefue basi situs; digiti scelidum digitis antipedum non longiores, nmues unguiculati; ungues validi, compressi, arcuati, acutissimi, in pedihus anticis quam in posticis fere majores; pori lemorales nulli; cauda trunco longior, teres, sensim in acumen desinens, integra.

Color: Corpus totum terreo-fuscum; collum, truncus et pedes supra, illud subtus quoque squamis aureo-flavis per maculas irregulares dispositis pulchre variegata; cauda fasciis latis aureo-flavis, in medio annulo terreo-fusco diremtis picta; abdominis scutella corneo-fuscescentia, plus minusve flavidovariegata. 
Proportio corporis parlium e mensura. Longitudo a rostri apice usque ad caudae finem

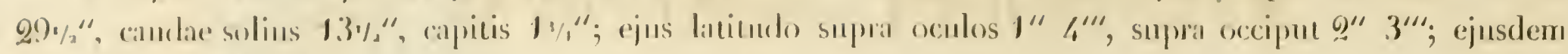

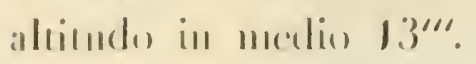

Mulilulio el vilue fulio. Habitat in Mesico, incolis ol, colporis structuram formirlabilem,

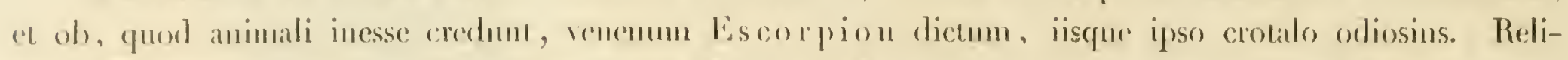

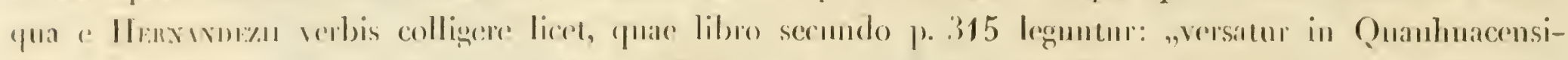

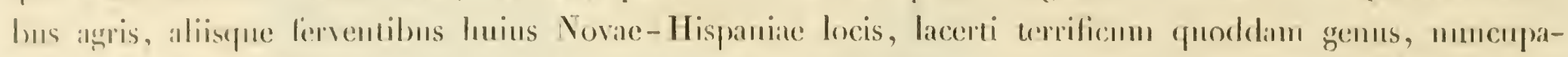

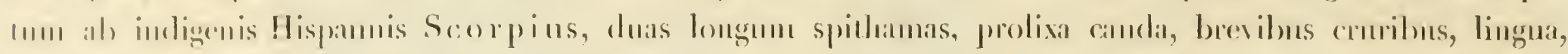

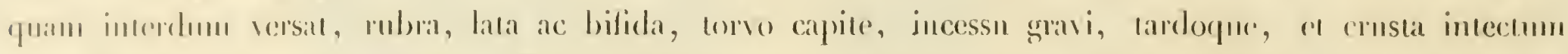

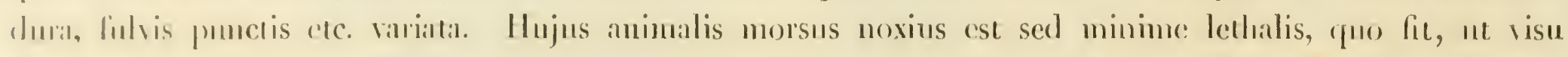

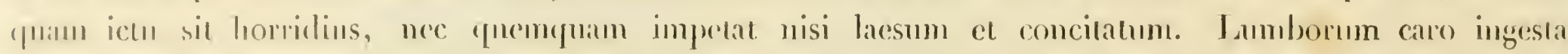

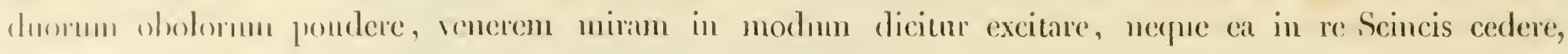
crustan vero Scorpionum adversari morsibus. Nec desunt gentes, quate arcus suos catem operiant muniant'fue, pulantes, ita venchatorum animalium nosiam naturam hebetari, atque coerceri.

OBSERVITIONES. Specimen depichum, muperrime necalum, nilidissimum, misit ad Musenm Berolinense cum rudjectis notis supra communicatis dom. DEPPE. In specimine effarcto linguae structuram auriumtue non satis examinare licuit. Secundum dentium stuamarumque formam ac posilionem ad lacertas thecoglossas Jj)stematis mei Heloderma referendum erit. HERNANDEZIT descriptio colorum ex bestiola viea ad specimen supra dichum plane quadrat. Ob squamarum duriliem color in speciminibus effarchis sice spirilui vini condilis vix pallescat. 



$$
\{\}
$$




\title{
AMPHIUMA DIDACTYLUM Cu.
}

\author{
Z W
}

\author{
T A в. XIX. F I g. 1.
}

\begin{abstract}
Character essentialis. Pedibus didactylis; corpore griseo-nigro, unicolore, subtus pallidiore.

Symonymia. Amphiuma means Garden Letter to Ellis, in the Corresp. of Linnaeus l. p. 399, to Linvaeus, l. c. p. 333. Sireni similis, Livv. to Gard. (Ed. Suith Corresp. of Linv. 1821.) Chrysodonta larvaeformis Mirchill, Medical Recorder 1822. p. 529. - Rich. Harlax: Journ. Acad. N. S. Phil. 1823. c. lig. ID. Ann. Lyceum Nat. Hist. New-York 1825. c. fig. - Amphiuma means (didactylum) Cuv. Mém. du Mus. d'hist. nat. XIV. 1827. p. 4. t. 1. f. 1. 2. 3. t.2. (Anatome.)
\end{abstract}

\section{E S C R I P T I O.}

Corporis forma universalis. Caput indistinctum, depressum, trunco minus altum, obtusum, lateribus rotundatum, longulum; maxilla quam mandibula paullo longior ejusque tomia cum labiis prominulis obtegens; oris rictus ultra oculos extensus, rectus; nares anticae, in rostri apice sitae, parrae, simplices, punctiformes, sibi approximatae; oculi capitis cute pellucida obtecti hinc inde absque palpebris, laterales, minuti, circulares, parum prominuli; lingua vix ulla; dentes simplices, conico-acuminati et subarcuati, approximati, in maxillis et in palato, hic quam in maxillis paullo minores per seriem $\Lambda$ - formem dispositi, maxillae viginti, mandibulae sexdecim, palati quatuordecim aut quindecim; a ures extus non conspicuae; branchiarum (?) fora men utrinque ad capitis latera postica oris angulo oppostitum, ovatum, subobliquum, in margine externo limbo, intus limbis duobus pusillis membranaceis, mobilibus, subtilissimis exstructum; truncus longissimus anguillae, teres, undique aequali crassitie et versus caudam vix angustior, a capite usque ad caudam plicis impressis, transversalibus auctus; pedes brevissimi, minuti, teretes, didactyli, postici anticis vix majores, hi paullo pone et infra branchiarum foramen siti; d igit i brevissimi, liberi, teretes, exunguiculati, externus interno parum longior; an us rima longitudinalis, simplex; cauda longula, sensim acuminata, acuta, compressa, in culmine superiore circiter a medio usque ad apicem carinata, subtus rotundata. Corpus totum undum, laeve, caput solum in parte supera subgranulosum.

Color. Animal totum griseo-nigricans, unicolor, in partibus inferioribus paullo pallidius; dentes aureo - flavi.

Proportio corporis partiume mensura. Longitudo a rostri apice usque ad caudae finem $10^{\prime \prime} 9^{\prime \prime \prime}$, caudae solius $5 \%{ }^{\prime \prime}$.

Habitatio et vilae ratio. Habitat in Novi-Aureliani, Floridae, Georgiae et Carolinae australis paludibus, in quorum limo in antris a se fossis, non raro duos aut tres pedes altis lumbrici terrestris in morem latitat; sed supra terram quoque per aliquot tempus vivere valet. Apud Nigros terrarum nominatarum. bestiola haec, maxime ab iis detestata, vocatur Congo-Snahe. Sunt specimina ultra duos pedes longa. 


\section{AMPHIUMA TRIDACTYLUM Gw.}

D) R E I \% E H I G E L R R A A L

$$
\text { 'T A B. XIX. F' I G. } 2 .
$$

Character essentialis. Pedibus tridactylis; corpore griseo-nigro, unicolore, subtus pallidiore.

Srnongma. Amphinma Lridactylum Cur. Mém. du Mus. d' hist. mat. XIV. p. 7. 1.1. f. 4.5.6.

\section{I) ES S C R I P ' T I O}

Corporis partes omnes ut in specie antecedente formatae ac pictae, pedes solum tridactyli. Longitudo y)", catudae solius $9^{\prime \prime \prime}$.

Il "bilalio el bilae ralio. Habitat cum praecedente specie.

O BSER VA'TIONES. Vidi Amstelodami hanc el praecedentem speciem cnm mullis aliis Amphibiis ex America septentrionali allatis in domo megotialoris aciarii. Jir ymi hestiolas emeral plmres mecum al relineandas ac describendas commmmicavil. 



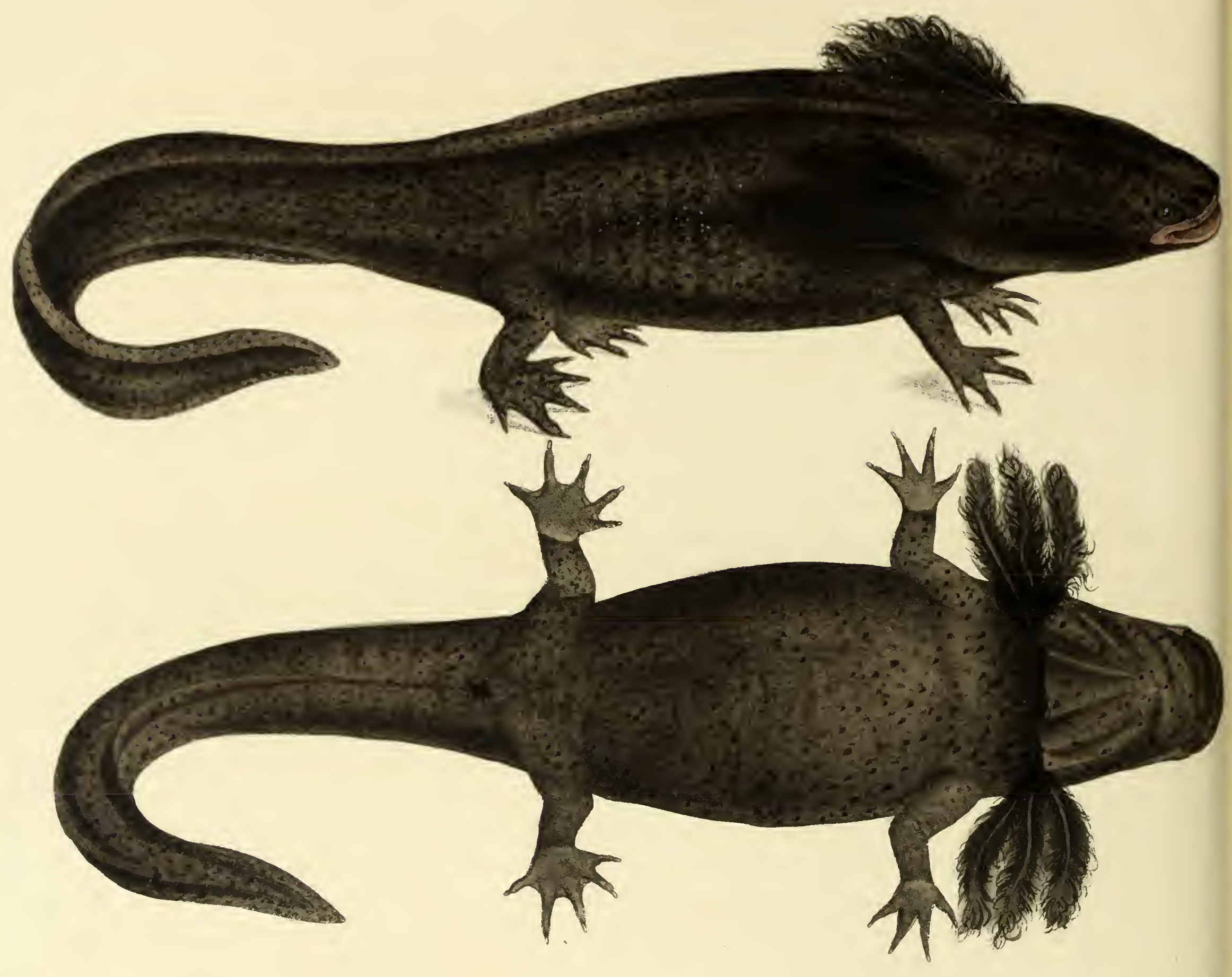

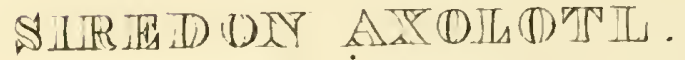

Tab:XX. 


\section{S I R E D O N A X O L O T L.}

\section{$\begin{array}{llllllllll}A & X & O & L & O & T & L\end{array}$}

\section{$\mathrm{T}$ a B. $\mathrm{XX}$.}

Character essentialis. Ex fusco nigricans maculis obscurioribus irregularibus nigris parvis, numerosis variegata; cute granulis parvis asperula; palmis tetra-, plantis pentadactylis.

Symonym. Axolotl sive lusus Aquarum Hernand. Thes. Nov. Hisp. p. 26 et p. 316 (exclusa adjecta icone ad Cycluram pertinente.) ID. Atolocatl l. c. p. 77. c. 4. Gyrinus mexicanus Shaw Naturalisi's Miscellany t. 343. ID. Siren pisciformis Gen. Zool. Vol. III. P. II. p. 612. t. 140 (fig. satis a ccur.) Axolotl Cuv. in Humb. et Boxpl. Observ. de Zool. 1. p. 109. t. 12. t. 14. f. $10-16$. Axolotl Ever. Hone Account of the organs of generat. of the Mexic. Prot. Philos. Transact. 1824. p. 419. t. 21. t. 22. t. 23. (figurae optimae.)

\section{E S C R I P T I O.}

Corporis forma universalis. Caput Siluri Glanis, depresso-rotundatum, latum, rostro obtusissimo, brevi, depresso-devexiusculo; maxilla quam mandibula fere brevior hanc non includens; oris rictus Siluri rectus, latus; tomia rectissima versus angulum oris exterius membra sublibera, laxa, maxillae descendente, mandibulae ascendente, utraque attamen apice antico ita connata ut infra mandibulae membranam cavum quodammodo nascatur, aucta; nares apicales, parvae, rotundae, simplices, introrsum patulae, largo interstitio inter se distantes, non breviori ab oculis; oculi parum prominentes, circulares, modice magni, cute communi capitis obducti, sed infra hanc pellucentes; a ures latentes; branchiarum foramen exterius maximum, usque ad mentum extensum, ibidem hujus cute laxa, libera in morem subligaculi obtectum; arcus branchiales ossei quatuor, latere interno cartilagineo-denticulati, tres eorum inferiores extus ramulis carnosis (branchiis) longissimis, in margine infimo in cirros numerosissimos excurrentibus, subulatis ceterum et longe ultra antipedes extensis aucli; dentes ranini, homogenei, nunerosissimi, parvi, simplices, angusto-conici, subtiliter introrsum flesi in maxilla et mandibula; palatini distincti, conformes, congesti, per lineanı arcuatan digesti; lingua parum mobilis, antice solum libera, cartilaginosa, apice truncata; tru ucus torosus, teretiusculus, ejus postica pars sensim angustior, antica occipitis latitudine; collum brevissimum, indistinctum; pedes quatuor perfecti, pro trunci mole imbecilles, tereti-compressiusculi, postici anticis validiores, pentadactyli, antici tetradactyli; digiti perfecti, subulati, depressi, acuti, basi, praesertim palmae, cute communi subconnexi, omnes mutici, apice vero subpellucido acutissini ac quasi huridi, longuli; palmae extimus cum intimo ac duo intermedii aequali fere longitudine, plantae omnes longitudine inaequales, quartus omnium longissimus; ca uda musculosa valida, compresso-lanceolata, supra ac infra membrana natatoria erecta, integerrima, superiore usque ad dorsi medium expansa, limbata, truucum longitudine aequans; an us rima longitudinalis simplex, marginibus copulae tempore perquam intumida ac rugoso - plicata.

Corp us totum nudum granulis cutaneis asperulum.

Color. Corpus totum fuscescenti-nigricans maculis numerosissimis parvis polymorphis, aigris variegatum.

Proportio corporis partium e mensura. Longitudo (speciminis modice magni) a rostri apice

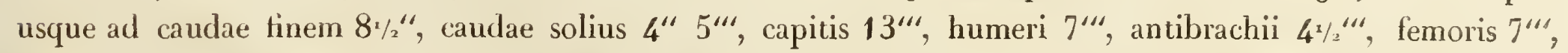
tibiae $4^{2} 2^{\prime \prime \prime}$, branchiarum ramuli supremi, omnium longissimi, 1 " $5^{\prime \prime \prime}$ infimi, omnium brevissimi, 10"; ambitus occipitis et trunci infra humeros $3^{\prime \prime} 1^{\prime \prime \prime}$, hujus ante femora $2^{\prime \prime} 10^{\prime \prime}$.

Wagler, Amphib. II. 
II abilllio "l pilar ralio. Habitat in Mexico, magun mumero in lacu juxta urbem Nexico sito

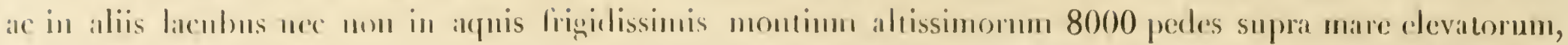

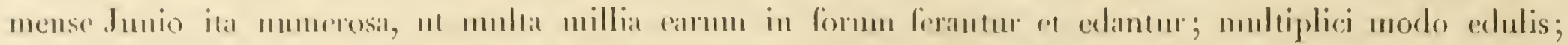

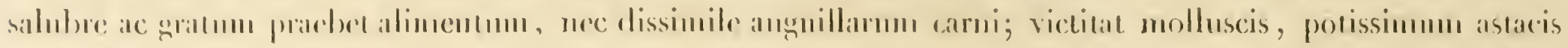
parvis"); copmlate tomprore ani regio, paresertim in matre, maxime intumescit; excrenenta non laro rubra; il) indigenis animalculum hoc vocitur 1,0 lotl.

()BSERVATIONLS. Specimina Siredonis Axololl adsunt in Museo Parisiensi, Berolinensi ac Monacensi: summus Lichtenstein tria mecum communicavit. - Colorum allerationem in speciminibus in spirilu rimi condilis lucusigue uondum observavi. Vidi nommulla 15 "longa. - 



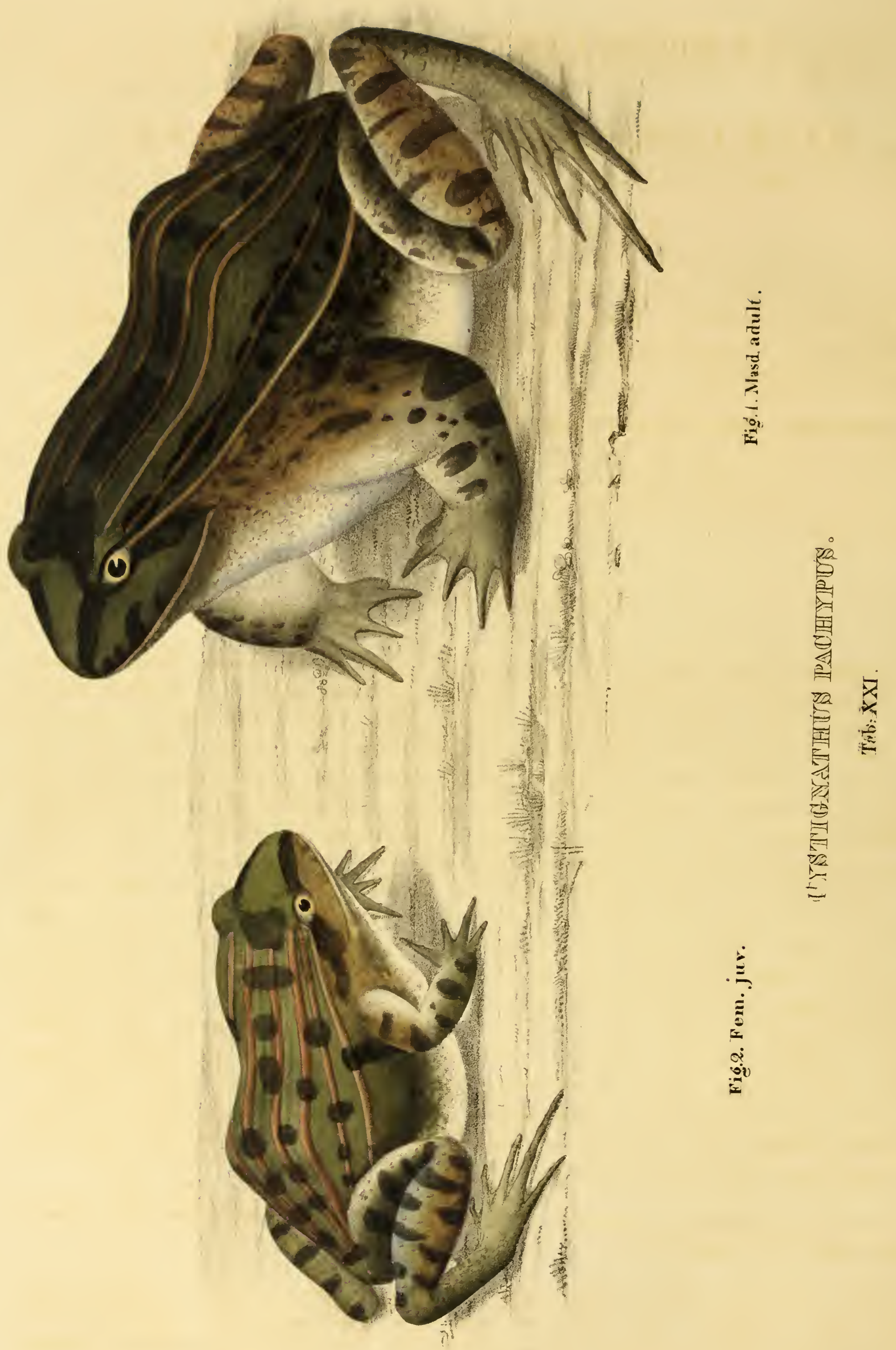




\section{I C K A R M I G E R L A D E N B L A E S E R.}

Character essentialis. Trunco supra olivaceo, nigro-maculato, vittis cutaneis longitudinalibus elevatis octo, sex intermediis fusco-rufis, interpositis lineis elevato-punctatis, subtus allo; antipedibus (maris) incrassatis.

Synonymia. Rana Gigas Sprx Ran. bras. p. 1. t. (foemina; descriptio e specimine detrito ac decolorato.) Id. Rana pachypus cum variet. p. 2. t. 2. f. 1. Mas. f. 2. Foem. (Figurae e speciminibus decoloratis ac detritis.) ID. t. 3. f. 2. (Jur.) Ib. Ralla coriacea p. 5. t. 5. f. 2. (Icon e specimine manco.) Rana pachypus Neuw. Beitr. zur Naturgesch. Bras. 1. p. 540 .

\section{E S G R I P T I O.}

Corporis forma universalis. Caput proportionatum depresso-ovatum, lateribus rotundatum; rostrum longuhum acutiusculum, in medio obsolete biangulosum; oris rictus maximus; lingua magna, plana, tenuis, rhombeo-circularis, in marginis postici et antici medio subexcisa, pone et ad latera libera sed non supra rostri apicem emissilis, papillis minimis, vix conspicuis, planis tecta, inde laevis; dentes maxillae unmerosi, homogenei, subapproximati, conici, palati dentibus maxillae forma similes, arcuato-dispositi, mandibulae nulli; nares rotundae, rostri apici proximae, sursum spectantes, angustiusculo intervallo inter se distantes, supra rostri proeminentiam subangulosam sitae; aculi modice magni, admodum prominentes auribus quam naribus approximatiores; tympanum modice nagnum, circulare haud procul ab oculis immediate supra oris angulum situm; truncus validus Ranaf temporariae vittis supra culaneis octo elevatis, quarum duae intermediae ab occipite, reliquae ab oculorun cantho postico usque ad femora ductae; puncta cutis elevata inter binas vittas per lineam digesta; reliqua trunci et pedis antici laevia; pedes postici verrucosi, anticis multo graciliores, pentadactyli, illi tetradactyli; palmae digiti breves, liberi, primus cun tertio, oumium longissimo, secundus cum quarto aequali fere longitudine, ommes largo intervallo inter se distantes; digiti prini phalanx prinia latissima, angulum a digito late distantem fornans; os metacarpi digiti ejusdem aequo modo dilatatum, palmae tuberum alterum formans; brachium et bresissimum antibrachium ingenti crassitie, teretia ${ }^{*}$ ); femora, tibiis breviora, tereti-compressinsculi, brachio multo graciliora; digiti longi, secundus, quartus ac quintus membranae rudimento inter se subconnexi, reliqui toto liberi, tertius quinti fere longitudine, quartus omnium longissimus, primus onmium brevissimus. Mas adult.

F'oe mina. Antipedes non incrassati, scelidibus graciliores, digiti primi phalanx prima atcue os metacarpi non dilatata; reliqua maris.

Color animalis adulti. Truncus, pedes et caput supra nigricanti-olivacea, subtus alba, plus minusve olivaceo-conspersa; vitta a naribus per oculos supra et ultra aures ducta; macula frontis inter oculos sita trigona, pone emarginata, aliaeque dorsi maculae oblongo-quadratac, quarum posteriores sensim minores, ad latera supra vittam elevatam penultimam irregulares et discretae, fasciolae breves in utroque fenoris tibiaeque latere nec non antipedum maculae nonnullae nigrae; vittae dorsi elevatae rufofuscae extima earum rubicundo-flavida; humeri et scelides nitore aureo-cupreo; pupillae margo aureus.

*) Os brachii dilatatum perfecte omoplatae tiguram aemulat. Vide meum Systema Amphibiorum.

WAGier, Axrutr. II. 
Color forminae junioris. Animali aduloo similis, corporis partes superiores guam in hoc dihtiores, maculae nighare unmerosiores, rotundatae; maxillac margo nombihil aureo-resplendens; vittae dorsi elevatae rulescenti-liscale.

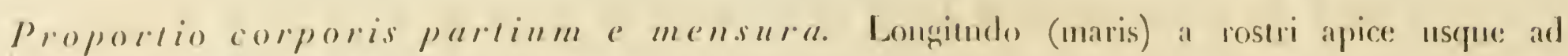

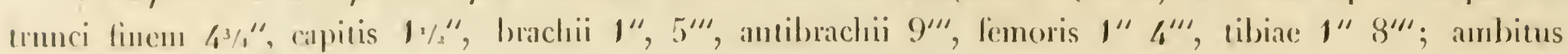

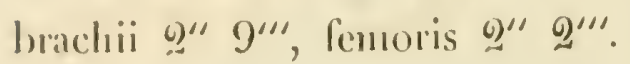

I/abilalio "l vilup ralio. Habitat in Brasilia ad flumina Espirito Santo et Jucu; vox phurium, qui coaxant, interdum non ingrata; timidus; ab indigenis ob femora, quibus vescuntur, capitur; alle salitil.

() BSERVATI(). Rana haec adest in Mheseo Monacensi ac in collectione mea. Exeniplaria nommilla communicavil unstrum Museum cum M. Lıgdunensi Bat. - In spiritu rini condilum animal tolum nigrical, si centrem exripis albidum. Notilias meliores de colore animalis viventis el de ejusdem vilae ratione debemus serenissimo principi MAX. NEUMTED. 
MCZ LIBRARY
MAVIRD UNIVERSITY CAMBRIOGE. MA USA 

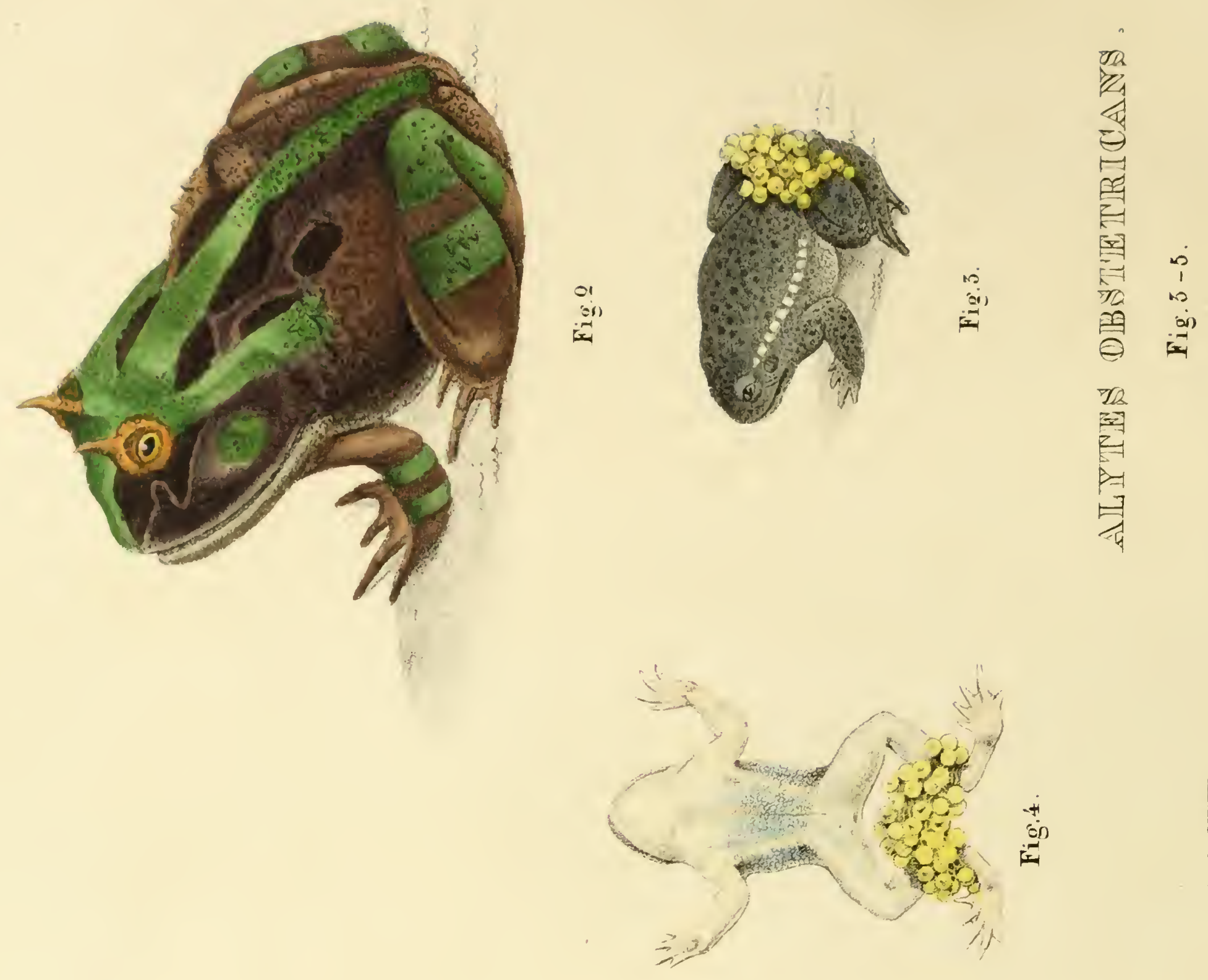

7
3
0
0
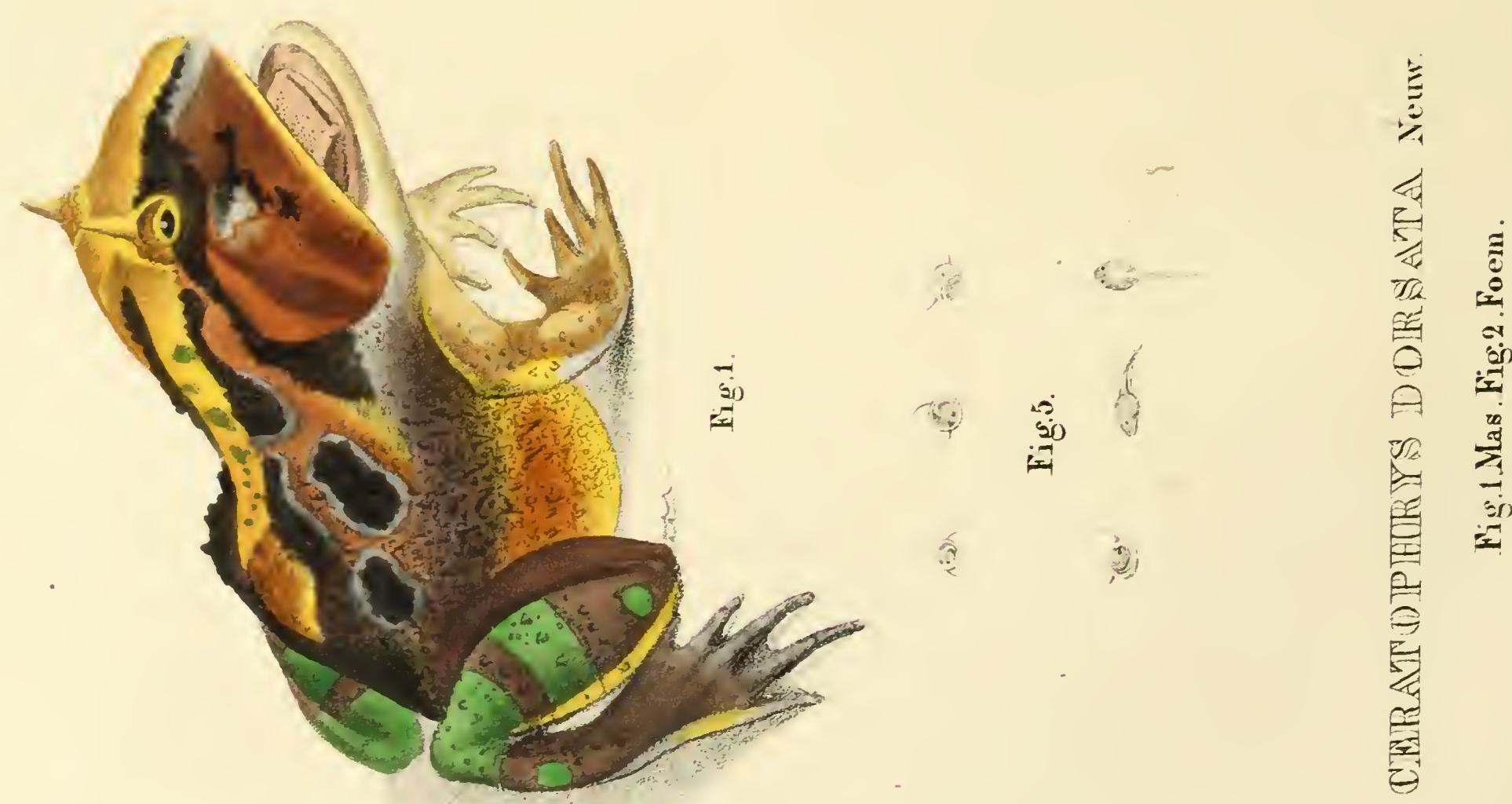


\title{
CERATOPHRIS DORSATA TEm.
}

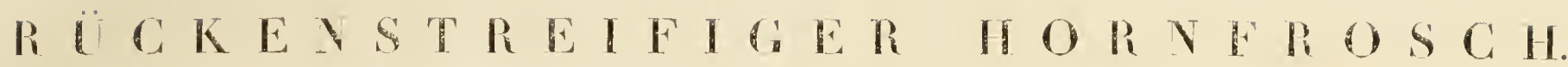

T А в. XYII. F г с. 1. 2.

Character essenlialis. Obscme fusca; villa docsi anumlaca (ua ris) sive siridi (fo eminae)

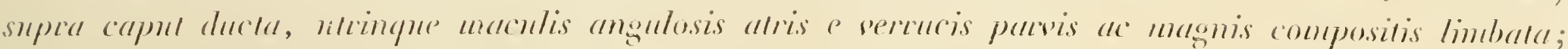
libiis viridi-bifasciatis; abdomime immacnlano; plantis semipaluantis.

Symonyma. Sebi Thes. 1. t. 71. 1. 1. Rana cornuta Liny. Mus. Ad. Fre 1. p. 48. Syst. Nat. 1. p. 356. Shaw Nat. Misc. t. 76. Merr. Teut. Syst. Amph. p. 176. 1. 14. Tules. Mag. der Gesells. naturf. Fr. in Berl. 1809. p. 92. (c. lig. mala.) Buf́ cornutus Laur. Rept. p. 95. Latr. Rept. II. 13. 117. Daud. Ran. p. 102. 1. 38. (fig. mala e specimine decoloralo Cerat. Daudini Cur.) Rana megastoma Sprx. Ran. bras. t. 4. f. 1. (Pict. e specimine decol. C. Spixii Cuv.) Ceratophrys dorsata Neuw. Beitr. zur Naturg. Bras. i. p. 576. ID. Abbild. zur Naturg. Bras. c. fig. opt. maris et foeminae e speciminibus vivis.)

\section{E S C R I P T I O.}

Corporis forma nniversalis. Caput ingens trunco multo latius ejusdemque fere longitudine, rostro longo, obtusissimo, latissimo a fronte versus apicem et ab oculis rersus tomia pierquam devexo; rostri niedium obsolete biangulosum eleratum; frons fere inter oculorum canthos anteriores foreolariimpressa, occipite multo altior; rertex planus, lateribus asserculiformi-prominens; oris carum ingens, richus usque infra aures extensus; lingua incrassato-musculosa suborbicularis, marginis postici medio sinuata, pone libera, emissilis (?), papillis minimis, planis tecta; dentes maxillae numerosissimi, homogenei, conici, subreclinati, mandibulae nulli, palati parvi (in animali adulıo nulli); margo tomiorum mandibulae integerrimus, acutus, apex ntrinsque denti-formi-ascendens; nares ad utrumque latus proeminentiae intermerliae rostri sitar, parrae, semilumblares, sursum spectantes, in medio oculormm canthum anteriorem inter et rostri apicen sitae; oculi altissime juxta frontis latera siti, orbitis clevatis; palpebrae inferioris rudimentun, superior completa, totmm oculum obtegens, in acumen cutanenm teres, gracile, corm recti formam aemulans terminata; a ures cute communi capitis tectae; rostrum glabrum regin parotica ac palpebra superior papillis nomullis tectae; truncus brevis, crassus, rersus femora subattenuatus, supra undique papillis cutaneis, conicis, erectis, mollibus ohtectus; dorsum superius in medio glabrum; caput, truncus et pedes subtus fere glabra, cutaneo-subgranulosa; antipedes proprortionati tetradactyli, scelicles antipedibus parum majores pentadactyli; digiti palmae toto liberi, teretes, tertius ommium longissimus, secundus brevissimus, primus a secundo largo intervallo distans, basi intumidus, ommes subtus glaberrimi; digiti plantae a basi usque ad medium palmati, membrana digiti extimi apicem attingens, inter digitum vero primum et secundum solum in basi conspicua, laevis; digiti omnes inaequali longitudine, quartus omnium longissimus, primus brevissimus, tertius quinto paullo longior, quarto multo brevior, ommes subtus glaberrimi sed, sicut plantae, infra articulationes verruca semiglobosa, glabra tecti; pedes supra, digitis exclusis, rerrucis, trunci illis conformibus, non creberrimis obtecti; lobulus cutaneus, pendulus supra anum.

Color. Mas. Obscure fuscus; vitta dorsi intermedia in rostri apice incipiens aurantiaca, nomnihil viridi-variegata; capitis latera humerique maculis striisque rubro-fuscis rariegata; maculae capitis vitta oculari nigro-fusca descendente diremtae; trunci latera maculis nonnullis virenti-nigris, limbo pallide rubicundogriseo cinclis; uropygium obscure fuscum virenti-lavatum; tibiae fasciis tribus eleganter viridibus; abdomen imnaculatum, flavido-album, ad latera ex fusco-rubro máculatum et punctatum; antipedes obscure fusci 


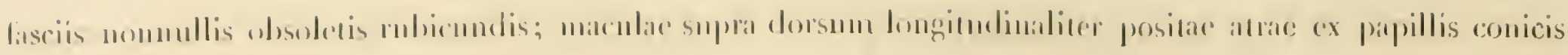
elongatis el multis aliis brevibus conflatare.

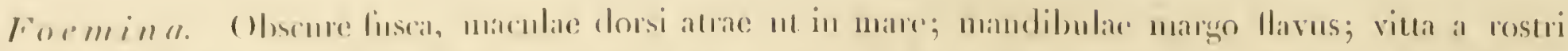

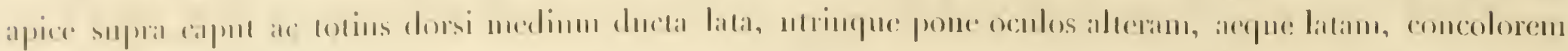

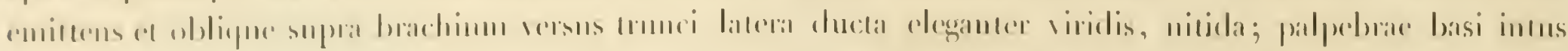

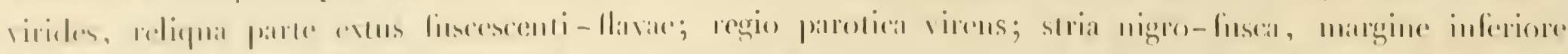

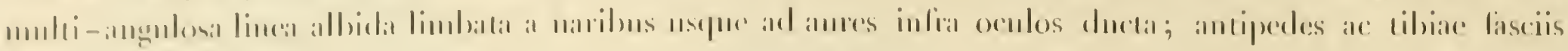

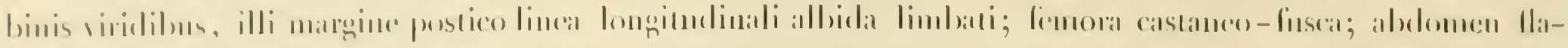

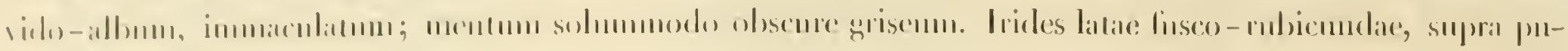

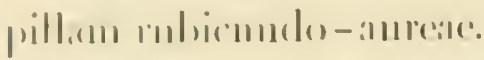

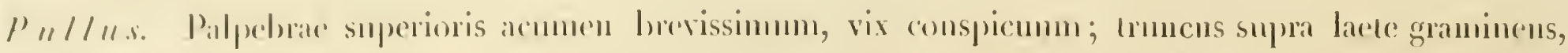

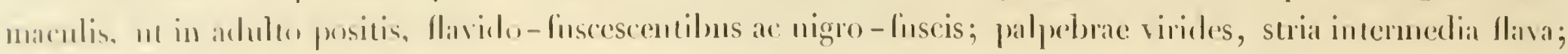

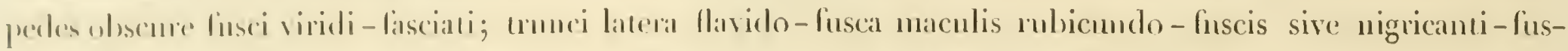
cis; partes corporis inferinris onnes sordide allare, immaculatac; verrucae nullae; naculae dorsi nigro-fuscae 111 in adulto clevatie.

Proportio corporis partium e mensura. Longitudo a rostri apice usque ad anum 4" 3²", "apitis g" g"', trunci 2" 5"', brachiii et antilsrachii 13"', femoris ac tibiae 1", 5"'; ambitus capitis prope oris angulum 6\%", trunci in medio 53/."; altitudo frontis $1^{\prime \prime} 4_{4}^{2} \%^{\prime \prime}$, occipitis $10^{\prime \prime \prime}$.

Habilalio elvilae ralio. Habitat in Brasilia a provincia Bahia usque ad prov. Rio de Janciro ac in Paraguaya. Degit in sylvis magnis, obscuris, humidis, praesertim in harum paludibus, undique, imo in sylvis ac locis cultivatis aridis saliens; victitat muribus, ranis, avibus, molluscis aliisque paris animalibus, resperi roce crocitante, monotona clamans; adpellatur Itannia sive Sapo de Chifre al, indigenis prope flumen Mncnri, Peruly peoc in Caravellas habitantibus, Kururú in provincia Bahia; apud Botocudos audit Engoallg. Neuw.

TOTA. Exemplaria Ceratophryos dorsatae exslant in Museo Parisiensi, Berolinensi, Monacensi; in spiritu vini condita tolo fusca apparent. Picturam animalis vivi hausi ex icone operis magnifici principis seren. NeUnIED. - Ceralophrys Boiei (NEun. Beitr.p. 59y el Abbild. sur Nalurg. Bras. C. granosa Crt.) species sane distincta, in Museo Berolinensi ac in collectione mea obvia. Sul nomine .Stombus cormutus"s descrilitur a GRArExmonst. (Isis 1825.p.952.) Differt a C. do rsata lobulo supra anum deficiente, plantarum digitis basi solum palmatis, abdomine constanter nigro-maculato, palpeürarum acuminibus longioribus etc. Dentes maxillae, a principe ron observati, ul in C. dorsala formati sunt. 


\title{
A L I T E S O B S T E T R I C A N S.
}

\author{
G E B L R T
}

\author{
'T A в. XXII. F г g. 5. 4. 5.
}

Character essentialis. Canus supra, verracarum juxta utrumque trunca latus albidarum serie, subtus albidus, mmaculatus.

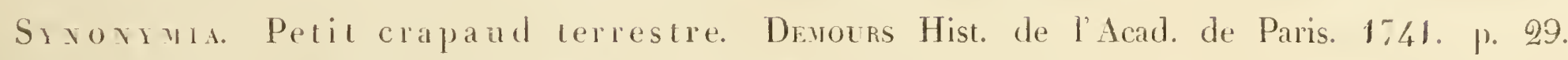
Bufo obstetricans Latr. Rept. p. 28. 128. Brongx, in Bullet. phil. 11. 36. p. 91. t. 6. f. 4. Latr. Salam. p. 40. Rept. 2. 1. 119. Daud. Ran. p. 87. 1. 39. f. 1. (lig. mala.) Rept. 8. p. 176. Geburtshelfer Kroete. Schran Faun. boic. 1. S. 272. Rama obstetricaus Wobr in Sturus Deutscht. Faun. III. Hft. 4. (duae figurae, non sat is accuratae.) Bombinator obstetricans. Merr. Syst. Amph. p. 179. n. 5. Bufo campanisonus Golof. Handb. der Zool. p. 484.

\section{E S C R I P T I O.}

Corporis forma universalis. Caput latiusculum admodum depressum; lateribus rotundatum; rostrum brese obtusum; maxilla mandibulae marginem includens; nares puncti-formes supra rostri apicem sitae, haud lato intervallo inter se distantes; oculi magni admodum prominentes auribus quam naribus proprores, pupilla triquetra; t pmpanum distinctum, subtile, circulare, haud procul ab oculis et immediate supra oris angulun situm, nodice magnum; dentes maxillae numerosi, homogenei, conici, subapproximati, palati dentibus maxillae forma similes per lineam rectam dispositi, mandibulae nulli; lingua lata, subcircularis, postice subtruncata, margine toto integra, tota mento affixa, postice vix libera, plana, papillis subtilissimis, laevibus tecta; parotis nulla; truncus depressiusculus, brevis, supra granulis minutis, laevibus, in utroque latere serie renucarum majorum laeium a brachio usque ad femur ducta tectus, subtus cutaneo-granulosus; an tipedes scelidibus multo minores tetradactyli; digiti breves, basi rudimento membranae inter se subconnexi; tertius corum omnium longissimus, primus extimi fere longitudine, secundus his paullo longior; planta pentadactyla; digiti semipalmati, quartus omnium longissimus, primus brevis, omnium brevissimus, secundus quinti longitudine ac forma, basi latiusculus, tertius his paullo longior.

Color: Caput, truncus et pedes supra cana, maculis parvis, discretis, fuscescentibus variegata; verrucae seriei lateralis albae; partes corporis dictae subtus albidae, immaculatae; irides perlaceo-albae.

Proportio corporis partium e mensura. Longitudo a rostri apice usque ad

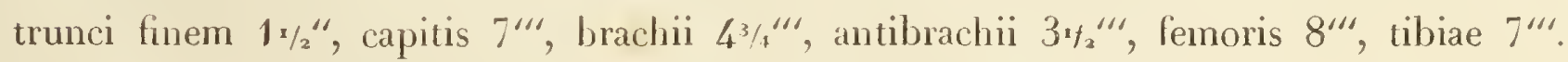

Habitatio et vitue ratio. Habitat in Galliae, Helvetiae et Germaniae regionibus meridionalibus, satis frequens in terra rhenana; rox sonum campanulae ritreae aemulans; copulatur sub finem mensis Martii; sub initium mensis Aprilis edit focmina ora 50 - 60,1\%" lata, flarida, mare cum pedibus adminiculante; tum foemina, mare ii alligato inter utramque tibiam utriculum cutaneum, ova includentem, in cellas subterrancas, pedem aut duos pedes altas, arcte clausas, aberrans de via defertur; in his usque ad certan ororum maturitiem latitat. Reliqua quae nunc sequuntur et ritam animalis spectant, dom. Dr. Lunuvicus Acassiz, vir doctus, historiae naturalis amore flagrans, in zoologia ac zootomia experientissimus, qui Alytent obstetricantem magno numero rivm vidit, mecum communicavit. lidem debeo multa specinina in spiritu vini condita ac icones ex animali viro.

Vagter, AmpHib. II. 


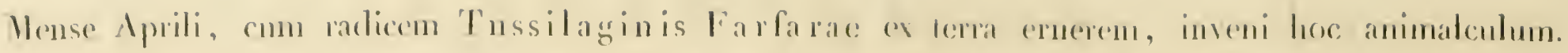

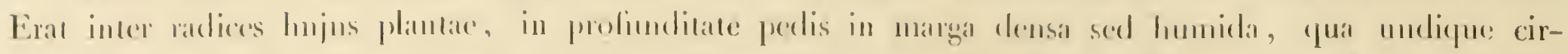

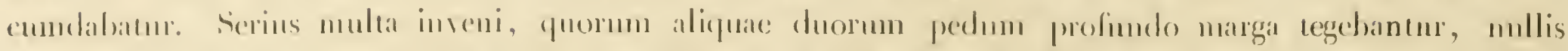

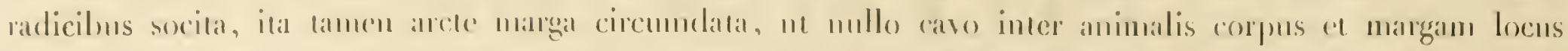

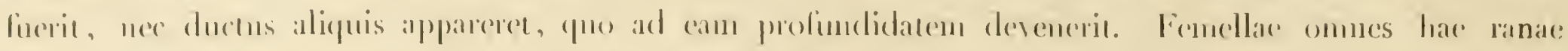

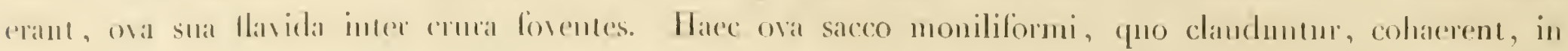
wormm interstitiis quasi articulato, coi lamen non allacent: nam si saccus in interstitie comprimatur, ora

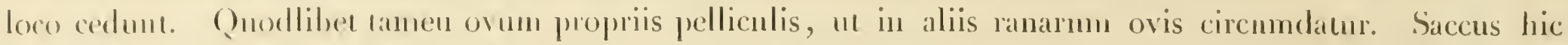
ergo pro condensilu phlegnate, quo aliarum ranarum ova obvoluta sumt, habendus.

Generatio verismiliter eodem modo peragitur, ac, in Bombitatore iggneo, amplexante mare lumbos

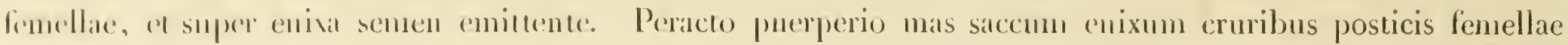

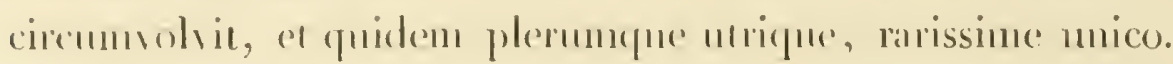

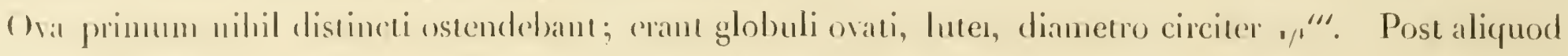
dies pellucidituten aliquan nanciscebantur; distinguebantur duo puncta obscura (oculi) et stria elevata longitudinalis. Pos plutes alios dies orat plane pellucida liehant, animalis partes explicabantur melius, os, nares, oculi, semicircularisyur syrini canda corporiapplicata manifeste videbantur; et paullo post etiant cordis pulsus el sangninis circulatio conspiciebantur; luteim oni tum valde diminutum erat; animalis motus augebatur, motns crant celeres, licbantque trudendo.

Post tres seplimanas na magniludime partorm pisorum nacta, pelliculae vero illa involventes ruptae sunt, animalibus sorum cavun omnino replentibus celeriterque sese et continuo agitantibus. Nunc etiam mater inquieta fiebat, omnique modo sese ovis liberare conabatur, redibat vero ad quietem affusa aqua. Nunc ora mox a pedibns crant soluta. Imposui illa in vas planum, aqua plenum, vidique animalia continuo fieri inquietiora, denique singuli gyrini fulguris celeritate suas pelliculas deserebant, mox attonita sistebant, denique natabant, cauda pro temone utentes.

Quac vero ora aquae non immisi, ab animalculis aeque deserebantur, sed gyrini mox emoriebantur, etian tum, quum illos nox a deserta pellicula aquae immisi.

Ex his colligi posse videtur, circa hoc tempus matrem subterraneam suam habitationem deserere solere, aquamque petere, in qua gyrini excluduntur. 
MCZ LIBRARY

HARVARD UIVIVERSITY

CAMBRIDGE. MA USA 


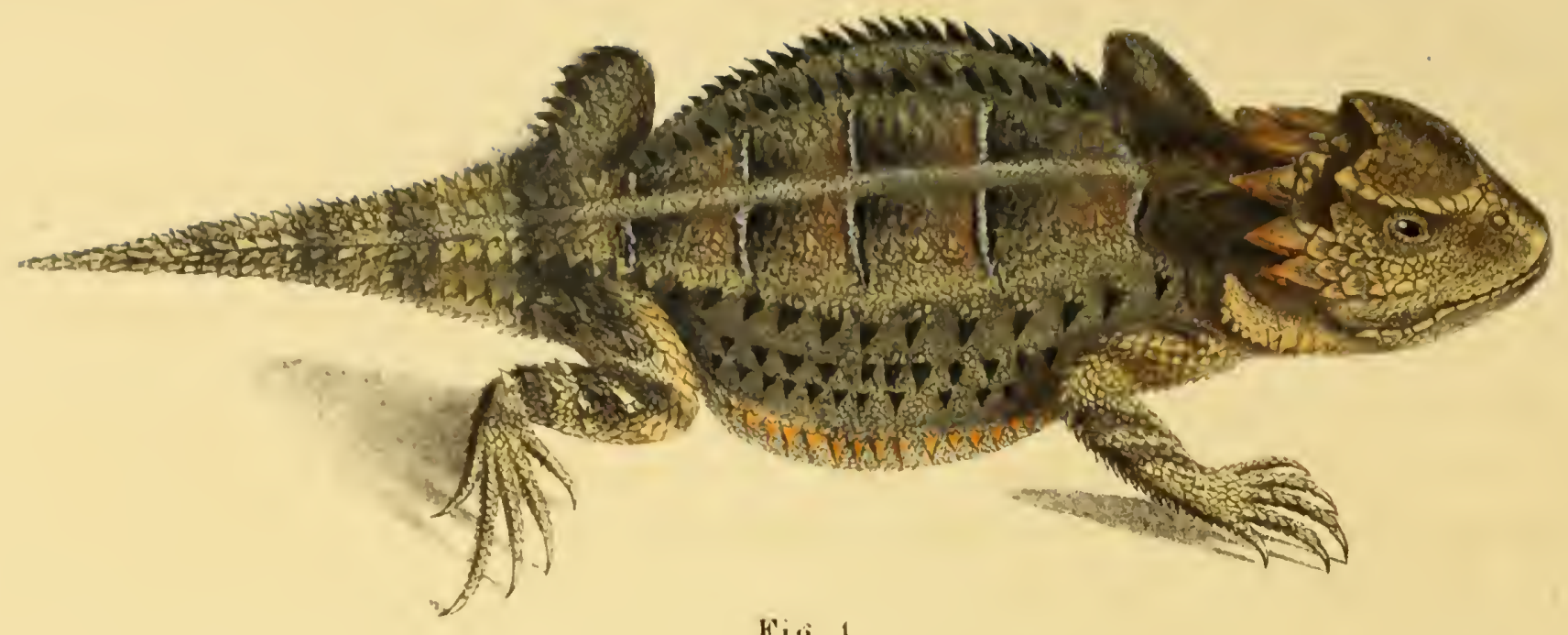

Fig. 1.

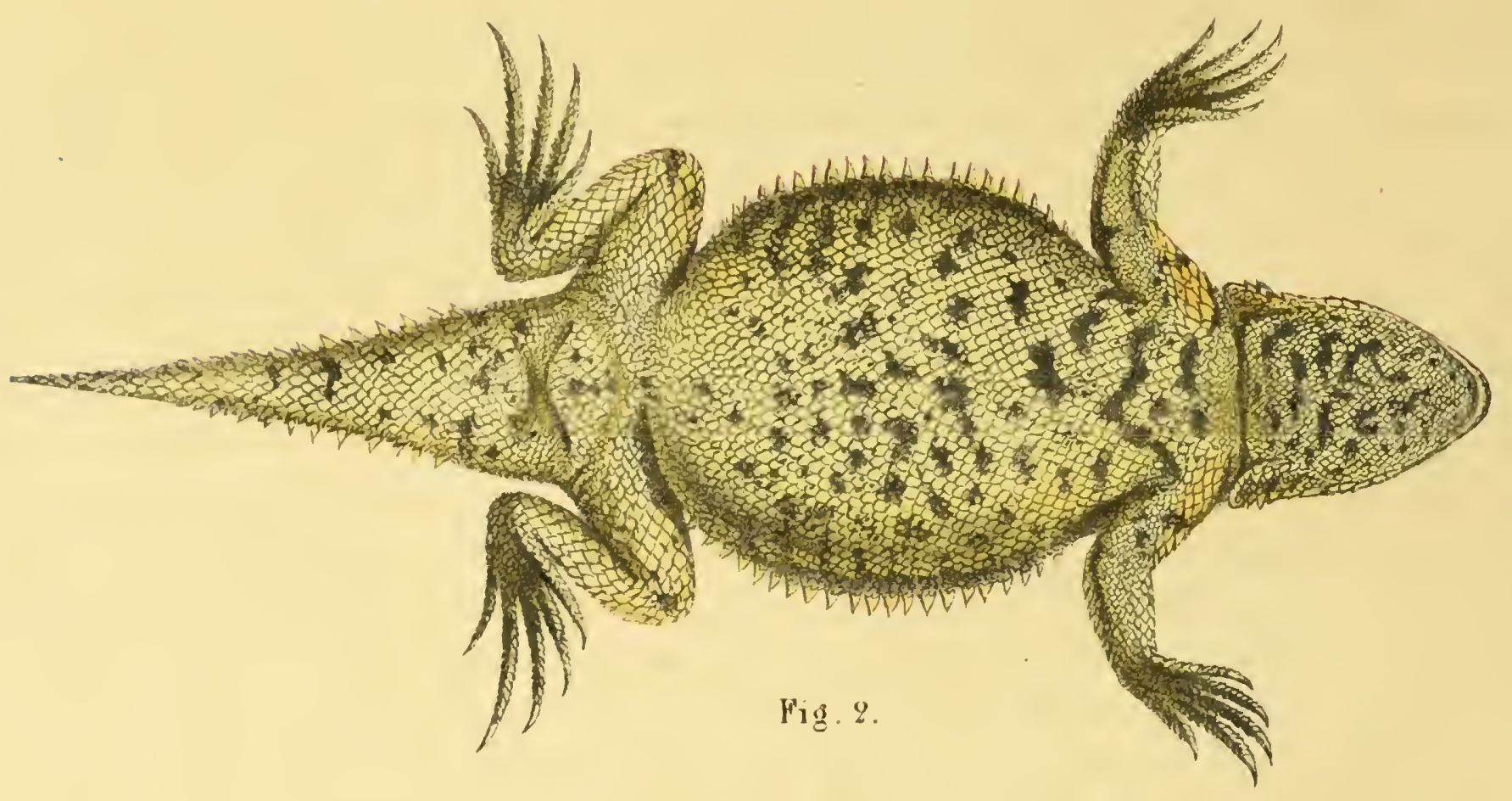

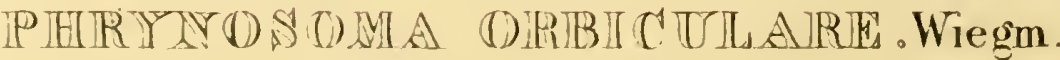

Tab. XXIII 


\title{
PHRYNOSOMA ORBICULARE wiegm.
}

\author{
K R E I S F Ö R M I G E R K R Ö T E N B A U C H.
}

\author{
T А в. XXIII. F I G. 1. 2.
}

Character essentialis. Occipite cormubus octo, trunco ad latera serie unica squamarum trigonarum, horizontaliter positarum cinctis; squamis abdominalibus laevibus; corpore supra cinereo, rubro-mixto, lineolisque transversis albidis quinque anucto, subtus stramineo nigro-maculato.

Symony 1. Tapayaxill Hermaxd. Thes. Rer. med. Nor. Hisp. 1. 67. c. 44 . Reccins ibid. p. 327. Cap. 16. c. fig. p. 327. et p. 328. Agama cornuta Harl. Jours. of the Acad. of natur. scienc. of Philadelph. IV. p. 299. c. fig. Phrynosoma orluiculare Wilgu. Isis 21. p. 367.

\section{E S C R I P T I O.}

Corporis forma universalis. Caput testudineum brevissimum, tetragonum, aeque fere altum ac latum, superins in clypeum cordis chartae lusoriae ad instar formatum, medio impressiusculum productum, in occipite et temporum lateribus series cornuum osseorum octo, retroversorum, conicorum, validorım, lateraliun sensim breviorum, discretorum arnatum, facie brevissima oblique truncata, conıpressiusculo-rotundata, temporibus osseo-intmmidis, regione ophthalnica impressiuscula; maxilla quam mandibula fere brevior, hanc non includens; lamina supraorbitalis ossea nulla; asserculum ante nares obsoletum; nares in asserculi apice obsoleto sitae, planae, rotundae, superius patulae; squanulae rotundatae margo, in qua sitae sunt, planus; oculi alte siti naribus quam auribus multo propioies, subobliqui, modice magni, palpebra utraque perfecta, crassa; aures distinctae immediate pone oris angulum conspicuae, tympano ovato alte sito; lingua crassa, lata, apicis rotundati medio incisula ibidemque latere inferiori papillis duabus laevibus, fere trigonis auctae; dentes primores ac anteriores maxillares inandibularesque simplices, conici, approximati, maxillae et mandibulae posteriores sensim majores, obsolete trilobi; palatini dentes nulli; truncus brevis, depressus, latissimns, fere orbicularis, inter axillas et ante fenora intractus; coll um brevissinmm, constrictum, infra distincte uni-, ad latera rugoso - plicatum; ruga lateralis validissima, sacculi speciem sursum ascendentis profundi formans; cauda dorsi longitudine, tereti-depressa, validiuscula, sensim acuminata, basi dilatata; pedes pentadactyli, proportionati, modice longi; digiti ralidiusculi, simplices, teretes, liberi, omnes, et palmae et plantae, longitudine inter se inaequales, palmae breves, primus quinti lere longitudine; ungues omnes magnitudine aequales, modice magni, compressi, subarcuati, acutissimi, subtus angulo in médio prominulo; pori femorales veri nulli, corum loco squamae in medio punctato-impressae.

Pholidosis. Squamae capitis ossibus quam maxime adpressae polygonae, elevato-punctatae et elevato-striatae; squamılarım labialium series unica; squamulae strictissimae, oblongo-quadratae, maxillae obliquo-, mandibulae recto-postpositae, laeves; nenti gutturisque squamae numerosissinae, rhombeae, laeves, mininae, partis inferioris trunci, pedum caudaeque majores plus minusve quadratae, aut rhombeae, omnes laeves, mmmerosac; menti sive ani seutella nulla, partes hae totae squamosae; squamae pedum ac digitorum superiores imbricatae, acute carinatae, colli trunci caudacque heterogeneae, minimae, conico-, plano-, rotundato-sive polygono-granulosae, intermixtis majoribus trigonis, compressis, plus minusve carinatis, supra caudan per series quatuor longitudinales, juxta trunci ntrumque latus per seriem unicam, supra dorsum per series circiter octo plus minusve regulares, supra femora per series duas dispositis. 
Color: Corporis partes inferiores ommes alliclae sive stranineae, maculis parvis, numerosis, singularibus, imrogulathus, nipris varegatac, superiores ommes cincreo-fuscescentes, restaceo-rubro nubilatae; dorsi medimm macnlis quatuor nigricantilus, lateraliter non distiucte, sed pone linea transwersa rubi-

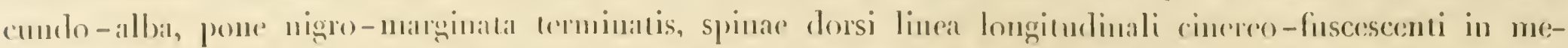

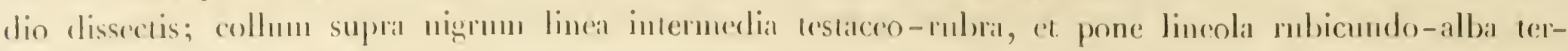

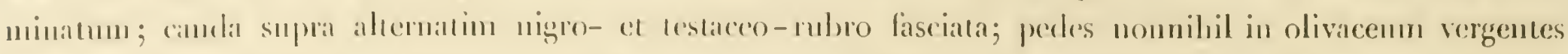

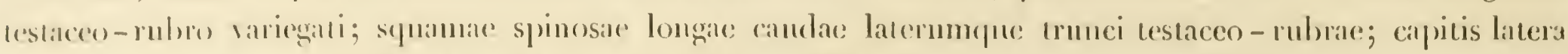
testices-rubro-varia; irides obscurate; mugues fisco-albae.

Proportin corporis parlinm o mensura. Longitudo a rostri apice usque ad caudae

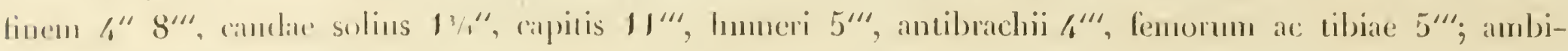

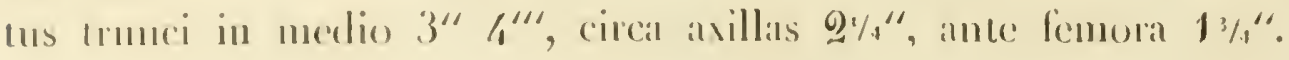

Il abilalio "l vilar lalio. Mabitat in Mexico inter lapides, colorem mutans. Reliqua vitae

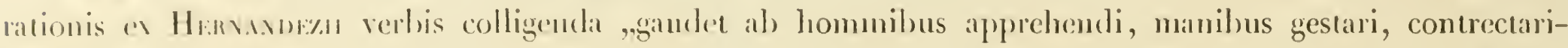
qure; placida fuadam securtate, ale trumpnillitate inmobilis, quamobren amicus homins ab indigenis numcupari solet. Hllul ast in ro mirabile, compressis vexatisve oculis, aut capite, aut parum indulgenter

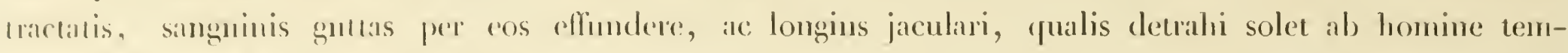
peratissimo, et salla fincenti valendine: lanto impetu, ut trium passum distantiam contingat trajectari. Vivit in montilus fregirlarmm regionum, ubi passim offenditur; gelidum semper tactu repertum, tardi incessus, ac raro multus focum, etian contrectatum atque apprehensum."

OBSEMVA'JU NiS. Speciem hanc habel Museum Berolinense el Museum Monacense; ad illud misit e Me. xico domimus J)ripl: ad hor baro de Kanmissir plura specimina optime conservata. Specimen purissimae conserualiomis dedil milui Museum primo dictum. - Color ruber speciminum in spirilu vini condilorum ac strmmineus mo.r prellescil; relipure colormm remanenl. - In oris cavo nonmullorum specimimum imeeni succum

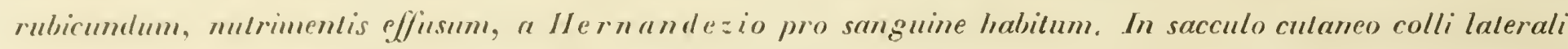
l.xorlis species gregalim degil. - Plir.nosoma orbiculare facile distinguilur a Phrynosomate bufonio (IIEG... Isis 1828.1).567.) simillimo stguamis corporis inferioribus laevibus ac serie unica squamarum majorum juxta abdominis latera. Agama cornula sane eadem species ac Phrj`nosoma orbicun lare; apices spnamarum ventralium emergenles facile pro serie secunda spuamarnm majorum haberi potnerunt. (Cfr. WIEGAAN l. c.) In hoc genere duae secliones occurrumt, quarum prima:

") squamis abdominalibus laevibus,

comlinel Phrymosoma orbiculare, ac Phr.jos. Douglassii (Ag. Dongl. BexL Transact.

of the Lim. Soc. 16. p. 105. 1. 10.)

allera syuamis abdominalibus carinalis,

phrymosomire bufomium. 


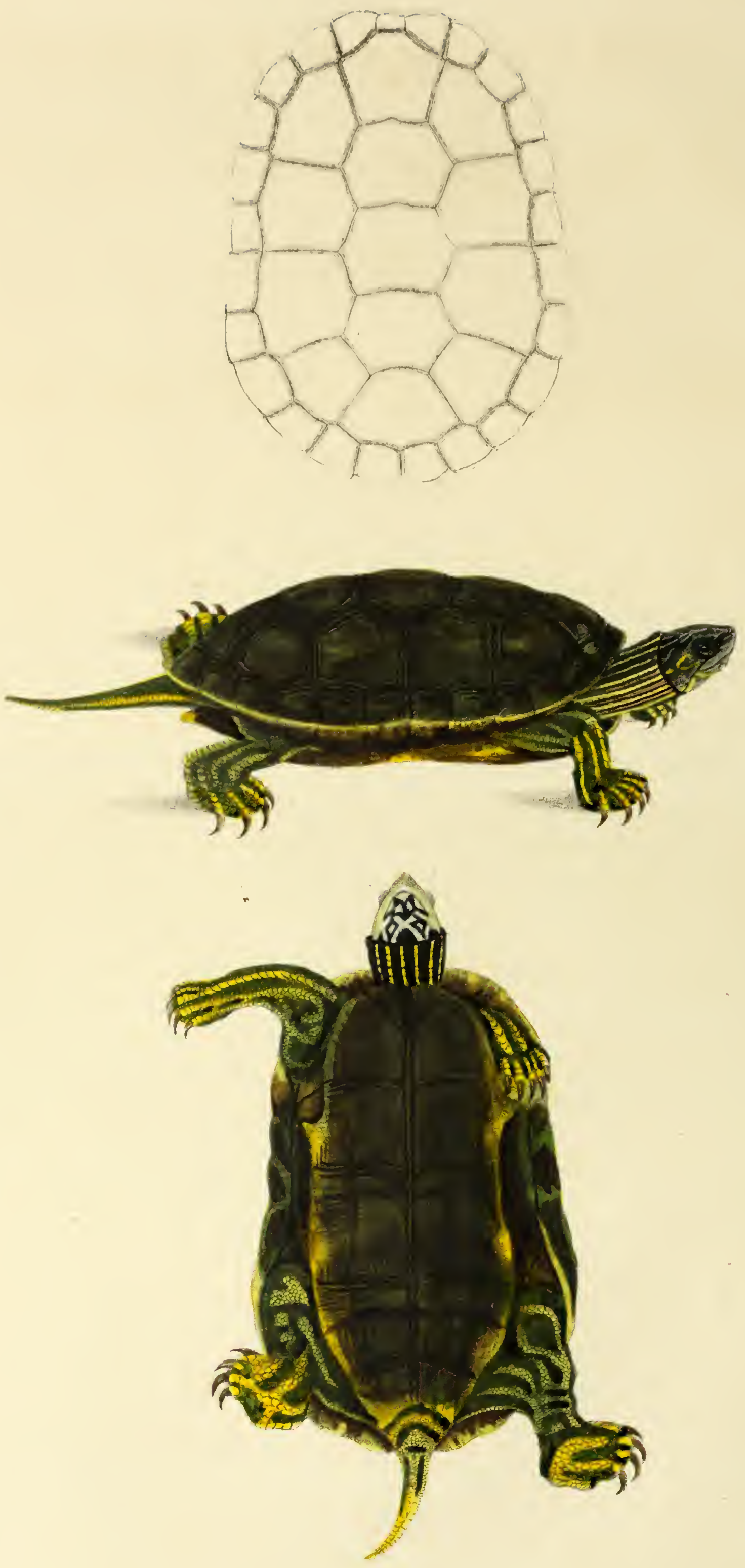

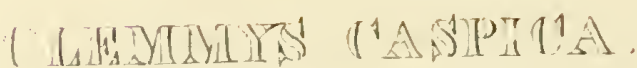

T:ab: XXIT 


\section{L E M M Y S C A S P I C A.}

C A S P I S C H E W A S S E R S G H I L D K R Ö'T E.

\section{T $\Lambda$ в. XXIV.}

Character essentialis. Testa humili, ovata, margine integro replicata, sordide olivacea, sterno apice postico emarginato nigricante, albido-variegato; collo, pedibus, cauda ac capite olivaceis luteostriatis.

Synonymi Testudo caspica Guel. Reise durch Rufsl. T. III. S. 59. t. 10. 11. (I)escript. et ic. pessim.) Gruel. Syst. Nat. 1. p. 1041. Schnemb. Schildkr. S. 344. Daud. Rept. 11. p. 104. Emys caspica Schweigg. Koenigsh. Archiv 1812. p. 306. spec. 21. et pag. 430. Emys lutaria var. \%. Merr. Syst. Amph. p. 25. - Emys scripta Schöpr. Schildhr. 1. 3. f. 4. 5. ic. mala e specinine sicco, pullo.

\section{E S C R I P T I O.}

Corporis forma universalis. Caput gracile, humile, planum, collo non latius, tetragonopyramidale, rostro subprodncto, acutiusculo, compresso, angnloso, pileo unica lamiua glaberrima tecto; rhamphotheca maxillae hanc mandibulae pysidis instar tegens, utraptue inlegra, acuta, superior narium latera attingens; nares simplices, parvae, rotundae; t ympanum conspicum, majusculum, ovalum; oculi subobliqui, proportionati, subproeminentes; mentum inerme; collum validum, depressiusculum, longulum, cute laxa ruguloso-granosa tectum sub testam prorsus reflexile.

T'esta ovata, humilis, margine toto integerrima, replicata, supra lỵpochondria subdilatata, supra (in animali juniori) distincte, sive (in animali adulto) obsolete tricarinata; carinae longitudinales, olsusae in scutellis vertebralibus omnibus, sed tantun in tribus lateralibus conspicuae; scuta vertebralia quinque, quorum anterius, omniun latissimum, pentagonum, secundım, tertium fuartumque magnitudine aequalia, hexagona, latiora quan alta, ultimun vertebralimm minimum obsolete hexagonum, sive potius pentagonum; scnta lateralia ntrinque quatuor, quorum primm atque nltimun irregulariter hexagona, reliqua, nlagnitudine aefualia, (juadrata; suturac horum scutorum onnes latinsculae, scuta ipsa margine subtiliter lineari-exarata; scuta marginalia laevia, viginti quinque, quorm anticum inıar angustum, quadratum, reliquis brevius, reliqua quadrata, prinum ntrinque prope scutum impar angustatum, omnia, impari scuto excepto, margine replicata; sternum cum lesta per symphysin connatum, magnum, thorace tamen multo ninus, apice antico olsolete, apice postico distincte sinuatum, ceterum integerrimum, laeve, planum aut parum concavunı (in foemina subconvexum), scutis duodecim integris, margine excepto, lineariexarato, planis tectum; hormu duo prima omnium minima, parva, triangularia, reliqua quadrata, quartum utrinque, dein quintum omnium maxima; in utraque juncturae fine scutellum quadrangulare.

Pedes robusti, anteriores compressi, posteriores tereti-compressiusculi, illi quinque hi unguibus quatuor armati, omnes rugulosi granisque cutaneis verrnciformibus in scelidibus, squamiformibus potius in antipedum latere superiore tecti, intra thoracen reflexiles; plantae pahmaeque verrucis graniformilous hispidae; digiti validi, supra scutellati, prorsus mol)iles, laterales sensim breviores, membrana laxa, lata, usque ad rbizonychii finem producta, robusta, margine semicirculari granoso-denticulata connexi; ungues robusti, longi, rectiusculi, teretes, acuti, subtus excavati; cauda dimidio sterno brevior, gracilis, teres, sensim attenuata, granoso-rugosa; an us infra caudae basin.

Waglen, Amphib. II. 
Color. Capmu nlivacem supra of ad latera lineolis longiudinalibus, mox apice anteriore convergentibus mudulifomibuspue, sive $\cong$ - Cormibus (in occipite) flavis ornatum; irides flavidae punctulo ante at pome pupillam nigricante signalae; colhum ad latera flavens, supra magis ad olivacenm inclinans,

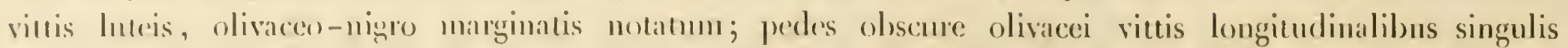
luteis picti; palma lasciis arcuatis luteis oruala; digiti luteo-maculati; caluda olivacent huteo-viltata; ster-

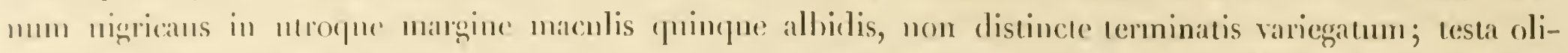

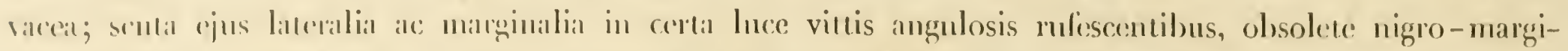

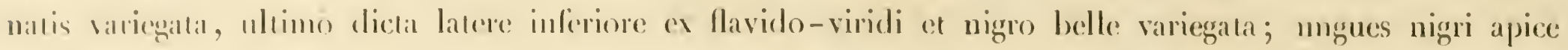
dilutiones. - In speciminibus hispanicis vittac capplis, pedum candaeque sunt aurantiacae.

Proporlio earporis purlium e mensure. Iongitudo a rostri apice usque ad caudae linem

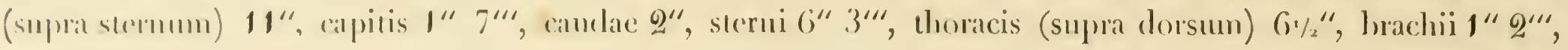

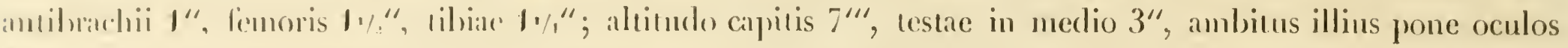

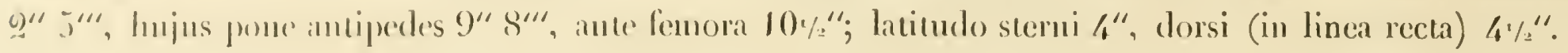

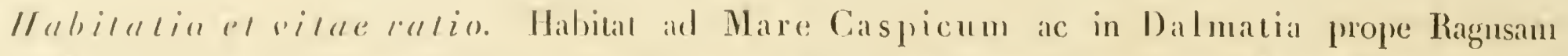

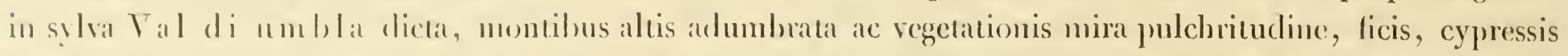

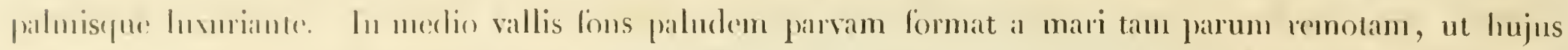
acressus et recessus in illa animadrerti possit. Hic vivit animal, et com homines adpropinquantes audiat repente sallando (puasi in apulm salit. Igile est et ambulans sive natans candam exserit et collum in altum

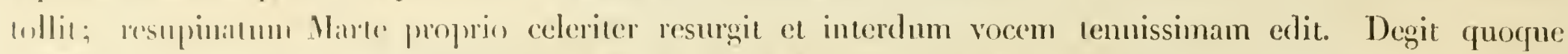
in Il ispan ia. (;visx, peregrinator, aftimat bestiam saepe tali magnitudine reperiri, ut tres homines ejus testae insistere prossint.

O B S E R VA'IO NES. Descriplionem ac ironem ex animali vion cum speciminibus duobus in spirilu vini us.erralis merum communicuvil dom. Mr. Carolus Michahelles, zoologine ac zoolomiae cullor indefessus, yui Ilalium superioren ar lerras illy ricas muperrime peragravil ibique non paucas res nalurales magni prelii collegilt. Specinen vinum ipse vidi. Nemo anle hos praeler GMELINUM hanc lesludinem ad nal u r am descripsil. Ilauseruml auclores capul squancalum ex GMELINT icone miserabillima. - Schoepfir Testudinem scriplam in Musco Parisiensi exslanlem ipse vidi. In specinuinibus pullis siccalis saepenumero videntur slrigae teslae obscuriores vario modo inter se connexae. Capilis, colli, pedum candaeque striae flavae animalis morlui mox pallescunt. 


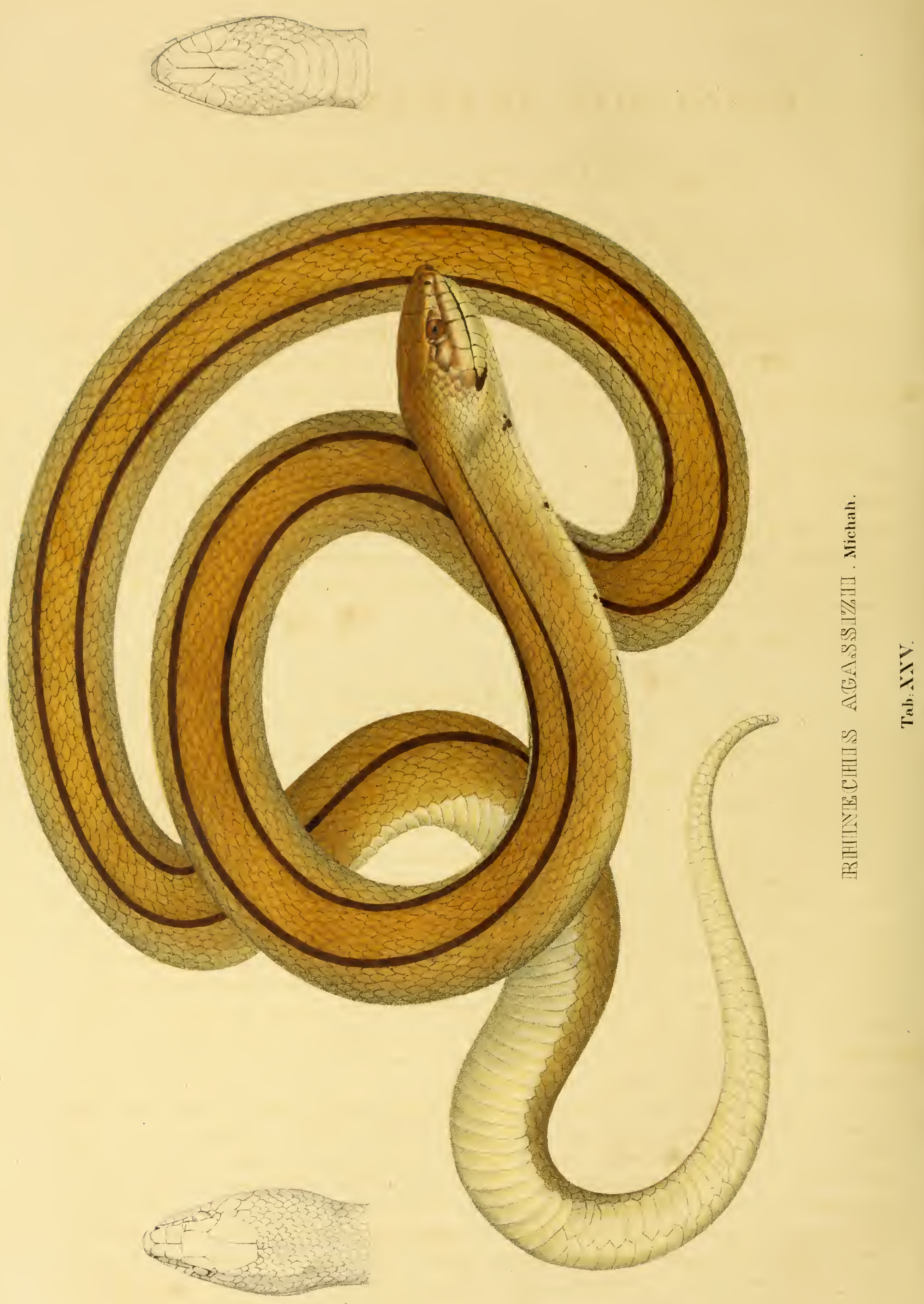


\title{
R H I NECHIS A G A S I ZII michahelles.
}

\author{
A GAS SIZ'S S CHNA UTZEN-N A T TER.
}

\section{T A в. XXV.}

Character generis. Habitus universalis atque pholidosis trunci Periopseos hippocrepis, capitis forma labio superiore acuminato, inferiorem longe supercedente insignis, scutum rostrale trigonum subtus medio profunde sulcatum, scutum oculare anterius unum, scula ocularia posteriora bina, pultus lateraliter levissime subsulcatus, dentes maxillares et palatini inter se homogenei, parvi, acutissini, retrorsum flexi, cauda brevis.

Character speciei essentialis. Trunco cylindrico, robusto a nucha usque ad anum eodem fere ambitu, squamis magnis laxe adjacentibus, oblonge rhomboidalibus, pone rotundatis, laevissimis, 27 in quavis serie obliqua dorsi medii, seriebus binis (scutis abdominalibus approximatis) reliquis majoribus, scutellis abdominalibus subangustis circiter 216, scutellis subcaudalibus serie dupplici alternante collocatis circiter 61, cauda in apicem obtusiusculam sese attenuante; supra colore leonino fulvo, infra flavescente, linea haud inlerrupta castanea in utroque dorsi latere a nucha usque ad caudae apicem protracta.

Sy nonymia. Nulla.

\section{E S C R I P T I O.}

Corporis forma universalis. Caput subdistinctum, versus apicem conico-acuminatum, mediocre, oris apertura a mento usque ad angulum recta, rictus ruber, lingua tenuis bifida, nigra, oculi laterales, majusculi, non prominentes, pupilla mediocris, rotunda, nares laterales, parvae a scuto frontali anteriori et a scuto nasali anteriori et posteriori cinctac, labium superius inferiori multo longius; scuta: scutum rostrale trigonum maxime prominens, subtus medio profunde sulcatum, scuta frontalia anteriora mediocria subpentagona, scuta frontalia posteriora magna, irregulariter quadrata, scutum vertebrale campaniliforme, basi lata, recta, scuta occipitalia magna, oblonga, margine externo curvato, irregulariter pentagona, pone ea squamae dorsales ceteris homogeneae, scutum temporale mediocre, subrotundatum, pone idem squamae ceteris majores, scutum loreum oblongo quadratum, scutum oculare anterius mediocre subquadrangulum, scutorum ocularium posteriorum bina, quorum inferius majus oblongo rotundatum, superius minus subrotundum, scutum superciliare mediocre oblongo-rotundatum, scuta marginalia labii superioris in exemplaribus nostris ${ }^{*}$ ) octona, quorum quartum et quintum inferiorem orbitae marginem format, primum rostro approximatum, parvum trigonum, alterum usque ad quintum subquadratum, sextum majus, angulum rectum postice linea curva clausum formans, septimum maximum subpentagonum, octavum mediocre, squamiforme, scutum labiale medium parvum, trigonum, scuta labialia accessoria tenua, lanciformia, scutorum nonorum marginalium labii inferioris primum minimum, sicut alterum et tertium subquadratum, quartum et quintum maximum, quintum postice rotundatum, sextım usque ad nonum mediocria squamiformia. Truncus longus, robustus, cylindricus, ab occipite usque ad anum parum sese attenuans, dein usque ad caudae apicem sensim sensimque acuminatus, undique rotundatus; squa mae magnae laxe adjacentes, oblonge rhomboidales, pone rotundatae, laevissimae; series binae scutellis abdominalibus approximatae ceteris majores, squamae versus caudae apicem hexagonae, scutella abdominalia numero 216 subangusta, oblonge-hexagona, paria dua ultima medio divisa, scucella subcaudalia numero 61 priora oblonga, posteriora regulariter hexagona in serie duplici alternante disposita, cauda brevis, octantalis, sensim sensimque abtuso acuminata.

*) Figura haecce, quam clarissimus praemature morte deraptus autor jam in lapide depictam reliquit, in hoc tantum puncto, a descriptione quam mihi pridem ex exemplaribus nonnullis desumsi, discrepat, novem ścuta marg. labii superioris repraesentans.

VVagler, AMphib. III. 
Color: Partes omnes superiores leonino-fulvae, inferiores flavescentes, paulisper in virile vergentes, a quovis muchae latere linea castanea ad vertebram latus usfue ad camdac apicem protacta excurrit, ad angulum oris parvi stria castancil, sitepius pone hanccem strian aliae parvae maculae castancac irregulariter collocatac.

Proportio corporis partium e mensura. Longitudo totalis a rostri apice usque ad caudace finem $4^{\prime}$, caudac solius $7^{\prime \prime}$, capitis $1^{\prime \prime} 6^{\prime \prime \prime}$, ambitus trunci medii $1^{\prime \prime} 4^{\prime \prime \prime}$, oculorum distantia a rostri apice $5 \% " 1 "$, labium superius prominet inferius $3^{\prime \prime \prime}$.

Habilatio et vitae ratio. Hispaniam neque non Galliam meridionalem confinem incolit, observationes accuratiores desunt.

OBSERVA'J'IONES. Primus dominus praecl. Dr. Walll ophidium huncce amoenissinum ex ilinere suo hispanico retulit. Prostant exemplaria in colleclione mea et domini praecl. Dr. Agassiz, porro in museis Vindobonae, Lugduni Batavorum el Turici.

Dr. M. 


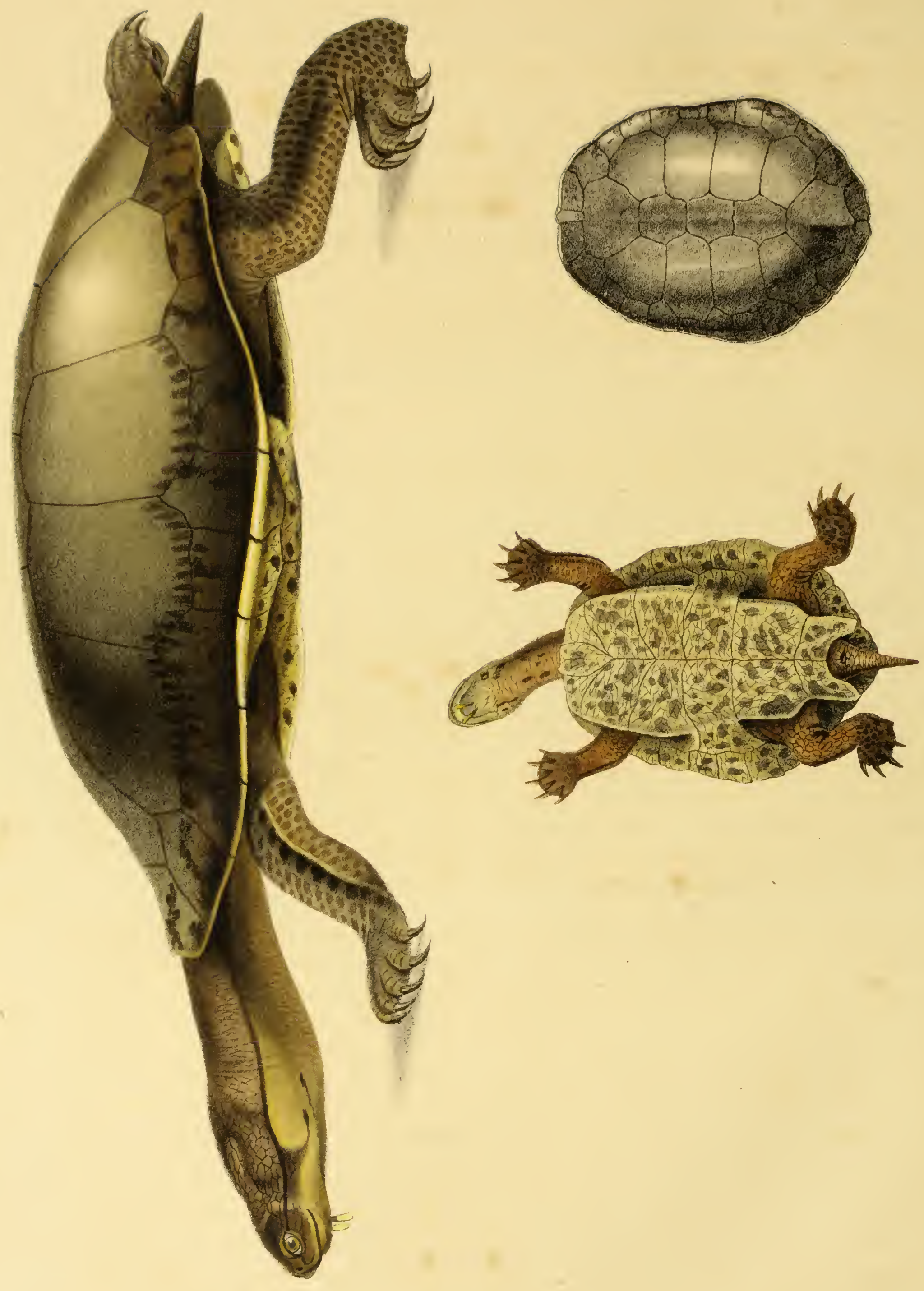

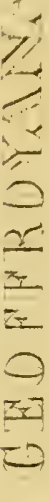

$-1 \quad$

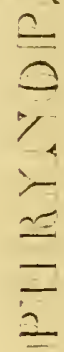




\title{
PHRYNOPS GEOFFROANA.
}

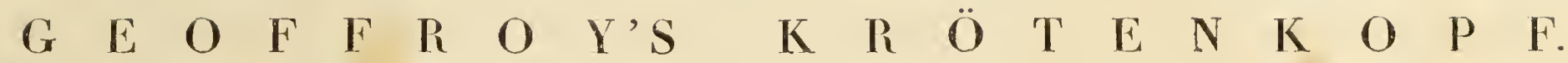

\section{T A в. XXVI.}

Character essentialis. Supra plus minusve obscure olivacea, obscurius submaculata, subuns flavida; testa obovata margine anteriore el laterali integra, hoc replicata, illo planiuscula, margine posteriore planiusculo-devexa quadri-sinuata supra spinam subplana scutellis subcarinatis; sterno apice postico quadridentato, antico truncato-rotundato, integro; collo capiteque supra olivaceis, subtus pallidioribus.

Symonyma. Emys Geoffroana Schweigger Koenigsb. Archiv für Naturwiss. B. I. 1812. p. 302. 350. Emys viridis Spix Testud. Brasil. p. 2. f. 4. t. 3. f. 1. (Testa a latere superiori et inferiori) Phrynops Geoffroana Wagl. Natürl. Syst. d. Amph. p. 135. Tab. V. Fig. 48 - 5 J.

\section{E S C R I P T I O.}

Corporis forma universalis. Caput bufonium, obtuse ovatum, a collo latitudine indistinctum, admodum depressum, planum, lateribus rotundatum, duplo latins quanı altum; rostrum obtusissimum, supra apicem mandibulae paullo productum; mentum planum, latum, angulo arcuato, papillis cutaneis duabus pendulis obsitum; gnathotheca cornea, humilis, lateribus convexa, laevissima, tomiis rectissimis, integerrimis, acutissimis maxillae introrsum flexis, tomia mandibulae includentibus; mandibula humillima; oris cavum latum; rhinarinm rotundatum, prominulum, naribus anticis transversali-linearibus; oculi subobliqui, mediocres, thinarium inter et oris angulum siti; a ures cute communi obtectae; collum elongatun, crassiusculum, depressum, lateraliter sub testae marginem recondendum, sicut caput corio prorsus nudo, impresso-vibicoso indutum.

Testa Obovata, humilis, latiuscula, supra lateribus devexa, supra spinam dorsi planiuscula, margine anteriore et laterali acutiusculo integra, hoc replicata, illo planiuscula, margine posteriore planiusculodevexo, subreplicato quadrisinuata, pars testae, regio supra cervicen et caudae originem convexo-compressiuscula; scutella disci tredecim, i. e. vertebralia quinque, lateralia utrinque quatuor, omnia glabra; scutella vertebralia latcralibus plus quam duplo angustiora; scutum vertebrale primum omnium maximum, permagnum, applanatum, medio longitudinaliter subgibbum, octogonum, in margine antico dilatatum, hexagonum, lateribus subarcuatum, in margine postico attenuato arcuato-emarginato, scuta vertebralia duo, sequentia oblongo-quadrangula, inargine subundulata, plana, longitudinaliter in medio carina obsoleta notata, horum posterius anteriori longius; scutellum vertebrale quartum omnium minimum lyriforme, antice ex utroque angulo in acumen angustunı (in specimine alio minus extensum) excurrens, pone recto-truncatum, angustatum; scutellum vertebrale ultimum subseptangulum, margine postico anguloso-undulato dilatatum, antico angustato recto-truncatum, lateribus obliquum, inedio compresso convexum; scutellum laterale utrinque primum scutellorum lateralium latissimum, pentagonum margine anteriore interuo oblique truncatum, secundum et quartunı quadrangula, angustiuscula, alta, ultimum nunc descriptis, latius, brevius, subsuadrangulum, margine inferiore anguloso-undulatum; bractéa marginales riginti quinque; bractea prima, media angusta, sublinearis, oblongo-quadrangularis, prima sequens utrinque quadrangula, sècunda pentagona, margine interno acuminato, onnes reliquae quadrangulae, margo quartae, quintae, sextae, septinae reflexus, octavae, nonae, decimae, undecimae subconcaro-deflexus, duodecima latere interno convexa; bractea nona et decima bractearum marginaliun onnium maximae,

Vagler, Amphin. III. 
decimae angulus posterior externns distincte prostans; sternum cum thorace per sympliysin latinsculam, super convexinsculam conuexum, lougnu, latiusculum, planum inpressionilus reticularibus in scutellis asperulum, lobo antico margine truncato-rotundatum, postico subangustato utrincpue ad latus anteapicem angulo prö̈minente, margine externo subreflexo, anctum, apice profinde sinuatum, unde bispinosum, scutellis tredeciun tectun; scutellum anterius medium magnum, distincte cordatum, antice subtruncatorotundatum, scutellum sequens utrinque primum, nunc descripto minus, triangul angustatum, magine antico arcnatum, secundum omnium scutellormn sterni maximum quadrangulum, margine anteriore obliquo, tertium secundo vix dimidio angustius quadrangulum, extus paullo dilatatum, quartum spadrangulum, quintum quartae forma, sed longius et margine postico olıliquum, cun angulo externo postico in acumen liberum desinens, ultimm utrinque distincte rhomboïdale; junctnra sterni scutellis duobus quadrangularibus tectum!.

Pedes modice longi, robusti, depressi, scutellis corneis, non contiguis, longitudinaliter dispositis, angulosis obsiti; margo posterior antipedum limbo cutanco, margine integro, superius scutis corneis, tenuibus tecto fimbriatus; digiti membrana lata, laxa, margine integra infra unguium basin extensa, palmati, omnes, excepto digito podariorum externo, unguiculati, ungues validi, longi, acutissimi, subarcuati; ca uda conica, testae marginem paullo transeedens, mutica.

Color: Caput, collum, pedes et truncus supra plus minusve obscure olivacea; testa maculis, plerumque lincaribus, praesertim supra marginem, obscurioribus, fuscis variegata; collum et pedes subtus dilutiora; testae latus inferius, jugulum et sternum flava, hoc nigrescenti-variolosum; stria linearis nigrescens ex oculi cantho postico versus colli latera ducta; irides...? ungues corneo-fusci.

Proportio corporis partium e mensura. Longitudo testae supra spinam dorsi 9", sterni $7 \% "$, latitudo testae supra medium sterni $6 \% 2^{\prime \prime}$, supra medium scuti vertebralis penultimi $7 \% "$, sterni supra medium scuti secundi $33 / . "$, supra medium scuti penultimi $3^{\prime \prime}$. - Sunt specimina maiora.

Habitalio et vilae ratio. Habitat in Brasilia ad flumen Amazonum.

OBSERVATIONES. Vidi et descripsi duo specimina effarcta, quorum unum, a cel. SCHwergGer l. c. descriptum, Museum Parisiense, alterum, optime conservatum, Museum Berotinense possidet. Ex hoc iconem praestantem amicissimus Dr. et Prof. WIEGMANN mecum communicavit. - Testa cum sterno, supra emessa, adest in Museo Monacensi. - Longitudo testae speciminis in Museo Parisiensi $1^{\prime} 22^{\prime \prime}$. Eiusdem descriptio, a cel. SCHWEIGGER exarata, satis accurata. 



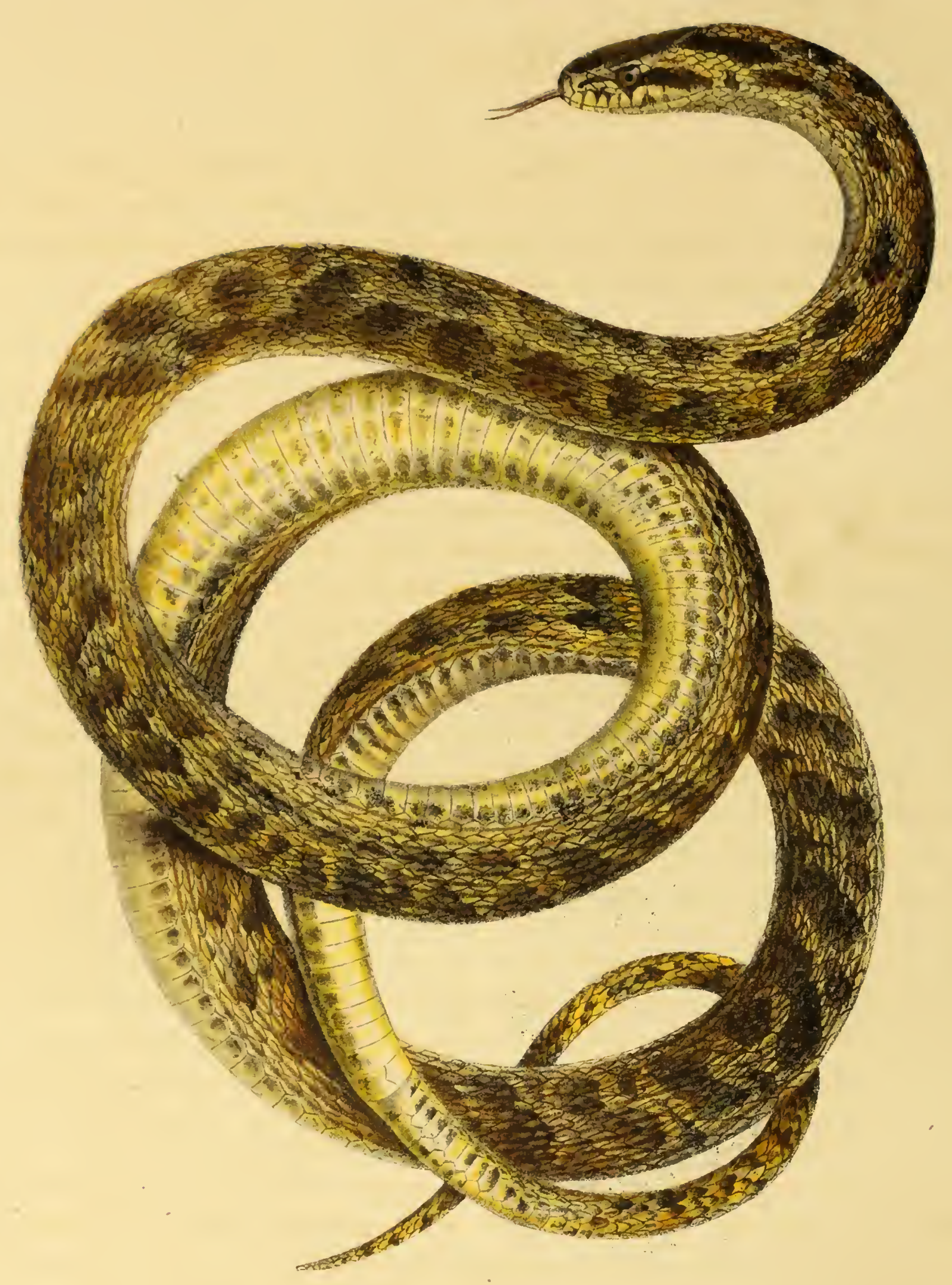

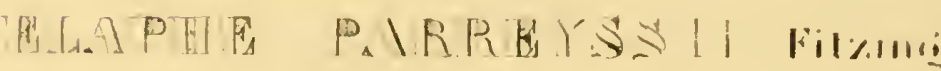

Tab XxVII. 


\section{PARREYSS'S ELAPHE.}

\section{T А в. XXVII.}

Character essentiulis. Notaeo fuscescenti-flavo, maculis fuscis, rotundatis, plerumque geminatis, longitudinaliter dispositis, variegato; vitta pone oculos oblonga, maculis nuchae duabus oblongis et pileo obscure fuscis, hoc obscurius fasciato; gastraeo flavescente utrinque serie duplici longitudinali macularum parvarum fuscescentium. Scut. abd. 220, scutel. subcaud. 63.

\section{E S G R I P T I O.}

Corporis forma universalis. Caput subdistinctum, oblongo-ovatum, supra planum, versus labia subrotundato-declive, rostro longulo obtuso, rotundato, lateribus ante oculos excavatis, labiis convexiusculis, maxillae superioris prominentibus; oris angulus versus occiput ascendens; rictus amplissimus; nares laterales, majusculae, in marginis interni apice scuti nasalis anterioris sitae; oculi laterales, congrui, medii, ovato-rotundati, vix conspicue versus rostrum deorsum inclinati; pupilla parvula, rotunda; lingua valde extensilis, bifida, nigra; dentes in maxillis et palato, solidi, aequales, parvi, acuti, recurvi, fusci; scuta: scutum rostrale magnum, medio convexum, infra emarginato-excavatum, versus scuta frontalia anteriora subtriangulum-terminatum; scuta pilei novem: frontalia an teriora mediocria, subpentagono-quadrangula, latere externo subrotundato; frontalia pasteriora anterioribus ultra dimidium majora, irregulariter quadrangula, antice latiora, postice angustiora; scutum verticale mediocre, scutis superciliaribus ultra dimidium majus, oblongum, quinquangulum, campanuliforme, pone trigonoterminatum, lateribus subtiliter sinuatum; scuta superciliaria mediocria, irregulariter quinquangula, antice attenuato-acuninata, postice latiuscula, declive truncata, margine externo supra oculos paullo prosiliente; scuta occipitalia majuscula, irregulariter sexangula, pone truncato-rotundata, margine externo sinuato; scuta nasalia duo, anterius majusculum, oblique rhombeum, postice naribus medio subemarginatum, posterius anteriori magnitudine aequale, quadrangulum, supra infraque truncatum, lateribus emarginatis; sc u tum loreu m hexagonum, angulis externis rotundatis; scut a ocularia an teriora tria, superius mediocre, in verticem ascendens, irregulariter quinquangulum, valde excavatum, inferior a minima, anterius quinquangulum, posterius sexangulum; scuta ocularia posteriora duo, parva, superius inferiore duplo majus, irregulariter pentagonum, inferius longius quam altum, rhombeum; scuta temporalia quinque, anteriora tria, minima, squamaeformia, posteriora duo, parvula, oblonga; scuta marginalia labii superioris novem: sex anteriora versus oculos introrsum flexa, primum quadrangulum, angulo interno acutissimo, secundum, tertium et quartum magnitudine fere aequalia, quadrangula, minora, quintum et sextum quinquangula, majora, subocularia, septimum anterioribus duplo majus, quadrangulunı, octavum irregulariter sexangulum, maximum, nonum antecedenti paullo minus, quadrangulum, supra angustius, infra latius; scutum labiale trigonum; scuta labialia accessoria rhombea; scuta marginalia labii inferioris decem: primum parvum, quadrangulum, secundum et tertium majora, irregulariter quadrangula, quartum longissimum, quadrangulum, quintum irregulariter quinquangulum, sequentia quatuor minora, irregulariter quadrangula, decimum antecedentibus conforme, minimum; scuta mentalia anteriora magna, posterioribus longiora atque latiora, sexangula; scuta mentalia posteriora angustiora, distantia; squamae gulares permultae, oblongae; scuta gularia nulla. Truncus incrassatus, subtoroso-gracilis, elongatus, fusiformis; collo occipite paullo magis tenui; dorso rotundato; abdomine subangulato-arcuato; depressiusculo, scutis 220. integribus tuto; superius squamis aequalibus, per 24. series longitudinales in dimidia corporis 
parte, versus caudam et caput per 20. series dispositis, obtectus; squamae lanceolato-rhombeae, dorsales laeviter cantrinatae, carin is in nuchalibus ac caudalibus squamis obsoletioribus, laterales series quatuor lieves. An us transversalis, scuto anali diviso. Cauda subtereti-fusiformis, a trunco subdistincta, attennata, acuta, longinscula, $1 / 6$ fere totius longitudinis serviens, scutell is subcaudalibus 63. omnibus medio divisis.

Color. Caput pileo obscure-fusco, nigro irrorato, postice, in margine scutorum occipitalium, late ex nigro-brunneo limbato; lascia inter oculos per scuta frontalia posteriora transversa, arcuata, nigrobrunnea, alterave per scuta frontalia anteriora ad oculos ducta dilutiore, lateribus flavescentibus, scutis marginalibus labii superioris nigro marginatis, vitta verticalibus fasciis concolore pone oculos, versus oris angulum dncta, latinscula. Nucha maculis duabus magnis, oblongis, ex nigro-brumneis, versus dorsum divergentibus, in utroque scuto occipitali incipientibus. Truncus supra ex fuscescente-flavus, maculis magnis, per duas series longitudinales alternatim dispositis atque interdum confluentibus, rotundis, ex brunneo-nigris, lineisque longitudinalibus fuscis, per medium singulac squamae seriatim decurrentibus. Latera dilution, utrinque serie macularum rotundarum, dorsalibus concolorum signata. Trunci pars inferior flavescens, maculis mediocribus, subtriangularilsus, nigrescentibus, in angulis scutorum et dilutioribus adjacentibus rotundatis, per duas series longitudinales dispositis.

Proportio corporis partium e mensura. Longitudo a rostri apice usque ad caudae finem $1^{\prime}, 7^{\prime \prime}, 6^{\prime \prime \prime}$, caudac solius $5^{\prime \prime}, 1^{\prime \prime}$, capitis $1^{\prime \prime}, 1^{\prime \prime \prime}$, ambitus capitis immediate pone nares $3^{\prime \prime \prime}$, pone oculos $5^{\prime \prime \prime}$, occipitis 6"', colli supremi $5^{\prime \prime \prime}$, trunci in medio 7'", prope anum $5^{\prime \prime \prime}$, caudae ad basin $4^{\prime \prime \prime}$, in medio $3^{\prime \prime \prime}$, in apice extimo $\mathbf{f}^{\prime \prime \prime}$, oculi distant a rostri apice $4^{\prime \prime \prime}$.

Habitatio et vitae ratio. Habitat rarissima in Chersoneso taurica provinciisque Russiac meridionalis adjacentibus, locis desertis arenosis, fruticosis, velox, timida, muribus victitans.

OBSERVATIONES. Dixi hanc speciem in memoriam celeberrimi Entomologi, amicissimi Domini Ludo. vici Parreyfs, qui specimen unicum, prope Nicolajew captum, ex itinere per Tauriam anno 1829 suscepto, attulit et vivum exemplar Museo Caes. Regio Vindobonensi libenter communicavit, in cujus vivario per duas menses conservabatur. In spiritu vini conditum parum pallescit.

\section{J. Fitzinger.}

Genus ,Elaphe" a cel. FITZINGER recens creatum mihi autopsia haud notum, Generi "Coelopeltis" postponi debet.

W. 



$$
2
$$




\title{
LOPHURA AMBOINENSIS GRAY.
}

\author{
H I E R O G Y P H I G H E R L O S E N S G H W A N Z.
}

\section{T н в. XXVIII.}

Character essentialis. Lateribus corporis squamulis parvis oblongis tectis, pedibus anterioribus et posterioribus squamis majoribus ovaliter acuminatis. Cauda corpore fere triplo longiori, supra alte cristata, figuris hieroglyphicis pictu.

Synomyia. Lacerta amboinensis, Schlosser Epistola de lacerta amboinensi. Amst. 1768. (Descriptio satis accurata et figura bene colorata). - Lacerta cristata, Bonnaterre Encyclop. XXXIII. Livr. p. 41. pl. 5. fig. 2 (Copia). Shaw Gen. Zool tab. 62 (Copia). Lacep. tom. I. p. 347. - Lacerta javanicà, Hornstedt Nov. Act. Stockh. tom. VI. fig. 1 mas et fig. 2 fem. (figurae malae). Deutsche Uebersetzung von Küffner p. 130. tab. V. fig. 1. 2. - Basiliscus amboinensis, Daud. tom. III. p. 552. Merrem. S. A. p. 46. - Hydrosaurus amboinensis, Kaup. Isis XXI. B. Heft XI. p. 1147. - Lophura amboinensis, Gray Plil. Magaz. and Annals of Philos. 1827. p. 54. Griffith. Anim. Kingsd. tom. IX. synopsis. - Istiurus amboinensis Cuv. Règne anim. II. ed. Deutsche Uebers. p. 60.

\section{E S C R I P T I O.}

Corporis forma universalis. Caput breve, obtusum, pyramidale, rostro plano, fere quadran. gulo, collum breve, corpus cristam versus compressiusculum, pedes anteriores et posteriores modice robusti, graciles. Partem tertiam anteriorem caudae crista ladiis 14-17 munita, supra dentata, antice humilis, pone alta, subito curve descendens obtegit. Cetera pars caudae lateraliter compressa, serra medio sulcata, elevata ornatur. Digiti pedum anteriorum inter se fere aequales, digiti pedum posteriorum inaequales. Ungues omnes acie inferiori bis excisi. -

Squamae capitis omnes rugosae, acuminatae, in vertice scutellum parvulum. Squamae corporis omnes parvae, oblongo quadratae. Cristae humilis sed profunde serratae, collum et crucem obtegentis, squamae obtusae. Squamae pedum ovaliter acuminatae, laeves. Squamae cristae caudalis, partem superiorem, posteriorem versus acute carinatae, supremae serram formantes. Ceterae caudae squamae sexangulae, carinis acutis in series longitudinales collocatis.

Color. Color principalis olivaceus et fuscus. Capitis vertex striis transversis subtilibus, cetera capitis pars pedesque maculis parvis nigro fuscis, latera corporis cristaque caudae figuris hieroglyphicis ornantur. Cetera cauda fasciis obscuris signatur. Crista dorsi viridi flavescens, ungues flavescentes.

Habitatio et vitae ratio. Vivit in Java atque Amboina, Valentino auctore (Descr. Indiae orient.) in aquis degit, quod vero Eschholzius, qui Lophuram pustulatam vivam observavit, valde dubitat, alteram Valentini notitiam, Lophuram bene arbores ascendere, Eschholzius affirmat. Cuvier in stomacho insecta foliaque reperiit.

OBSERVATIONES. Cel. Cuvier nomen Lophuram in Istiurum mulavit, quippe nomen Lophura nomini

Lophyro similius sonat. Equidem in Iside demonstravi, nomen Lophyrum jam pridem a Poli testaceis quibusdam, a Latreille piezatis et a Vicillot avibus quibusdam tributum fuisse neque iterum amphibiv Wagler, Amphib. III. 
ullo dari posse, nomen "Lophura", yuod cel. Gray Saurio huicce contribuil, milhi hisce rationibus haud inidoneum videtur, nomen genericum Cuvieri "Lophyrus" (agama gigantea) vero ïsdem rationibus duclus in Goniocephalum mulavi.

Generi Lophurae Gray plures species admumeral, e.g. Plyrsignathum cochinchinensem pluresque novas ul Lophuram C'uvieri e Cochinchina, Lophuram Lessuenri e Paramalla. Istiurus pustulatus, Eschhol:. zool. Allas tab. 2. poris femoralibus carel, crislayue characteristica in naso ornalur. Valde similis ille saurius est, "ui in museo Lugdunensi numerose conservalur, ex. quo eliam notilias in observalionibus de Merremii Sy-stemate (Isis 1825 . Heft 5) desumsi. Saurius ille dentes quinıue in osse intermuxillari habel, crislaque caudali in prima juventule carel.

Si comparalio horum duorum sauriorum cum typo generis Lophurae alias quorpue differentias ostenderet, nomen Isliurus hisce duobus tribui posset.

Dr. Ka $a u p$. 


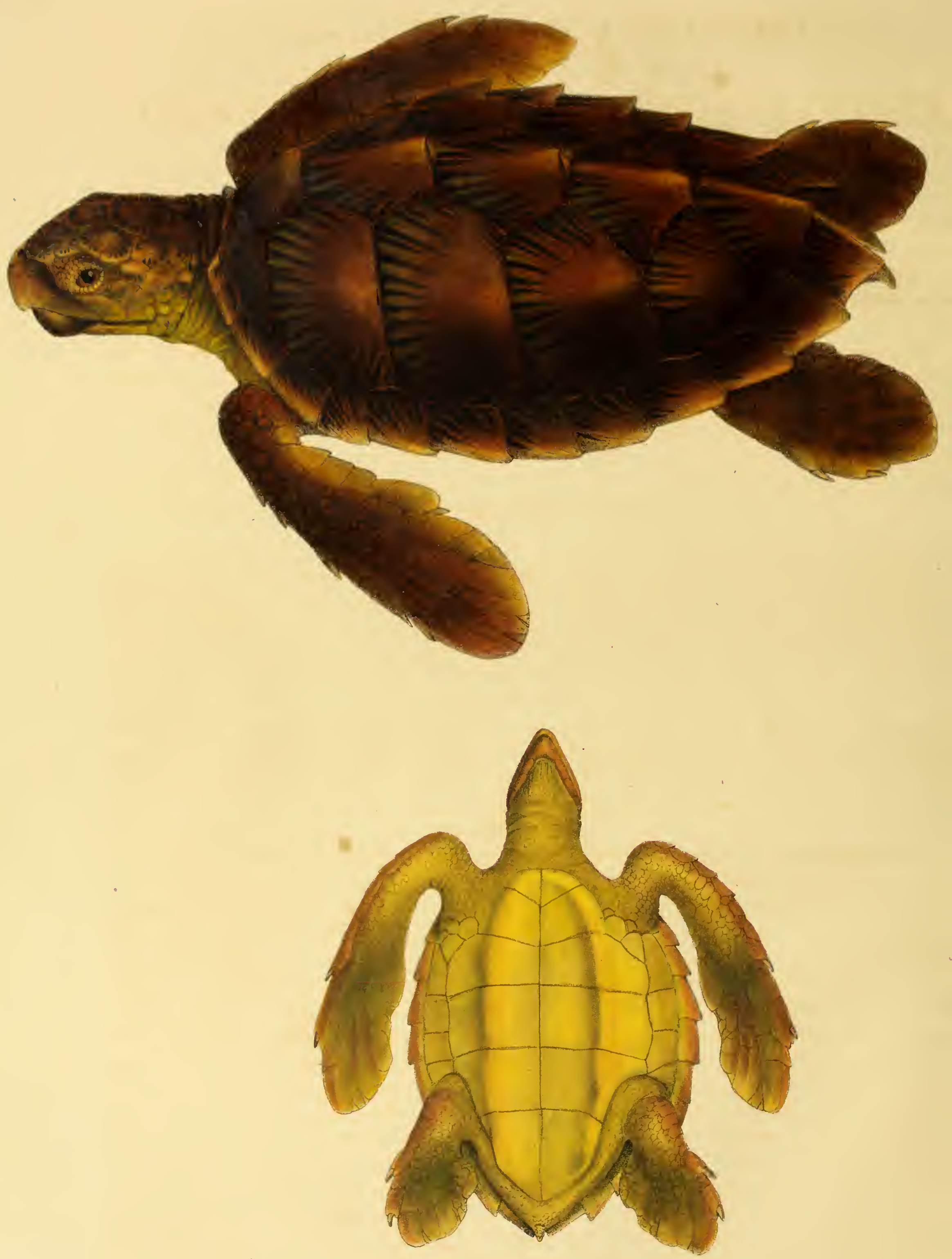

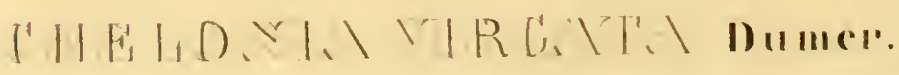

Wab: XXIX. 


\section{S T R A H L E N C H U P I G E S E E S C H I L D K R Ö T E.}

\section{T A в. XXIX.}

Character essentialis. Testa (animalis adulti) suborbiculari sive (animalis junioris) cordatoovata; margine dentata, castanea supra, scutis fulvo-ac nigro-radiatis, subtus flava.

Symonyia. Caretta, or Sea-Tortoise Bruce Voy. V. p. 215 (cum fig. animalis adulti a latere superiore). Testudo semimembranacea Heris. Observ. Zool. ed. Hammer p. 219. (Descriptio animalis junioris). Testudo Caretta ß Daud. Rept. II. p. 48.4 Chelonia virgata Dumer. in Mus. Paris. SchweIgg. Königsb. Arch. für Naturw. 1812. p. $291,411$.

\section{E S C R I P T I O.}

Corporis forma universalis. Caput subdistinctum, cordato-ovatum, ante et prope oculos admodum compressum, supra frontem angustum oculosque convexiusculum, hinc inde ad nares declive, ad tempora planum, occipite latius quanı altum; nares supra marginem V-formem gnathothecae sitae, angustiusculae, verticali-oblongae, mediocres, mesorbinio latiusculo, subconvexo; maxilla superior inferiorem pyxidum instar includens, tomiis rectis, integris, acutis ante apicem obsolete sinuatis, dertro compresso-convexiusculo; angulus mentalis latus, distincte biangulosus, gonyde lata, ascendente, convexa, in uncum desinente, lateribus plana, tomiis acuta, integra; oculi maiusculi, palpebrarum apertura admodum obliqua, palpebris latis squamoso-rugosis cincti; palpebra superior inferiore multo latior; aures scuto magno tectae, unde extus, nisi in speciminibus siccis (hic in forma foveolae), nullo modo conspicuae; collum breve, nudum, supra solum impressionibus, squamulas quasi efficientibus acutum, subtus obsolete verruculosum. Testa (animalis junioris) cordato-ovata, longitudinaliter in medio alte convexo-carinata, lateribus admodum declivibus, margine anteriore supra collum recto, supra brachia lineam obliquam, integram formante, glabro, hinc inde ad caudam horizontaliter prostante et ad scutorum angulum posteriorem externam emergentem dentato; scutella disci sexdecim, vertebralia sex, lateralia utrinque quinque; scutella omnia cornea, contigua, glaberrima, juxta suturam obsolete lineari-exaratis; vertebralia, excepto scutello primo brevissimo sed latissimo, pone recto in utroque vero latere trigono-truncato, medio alta carina, pone in acumen altum distinctum subtrigonunı excurrente aucta, magnitudine inaequalia; scutellum vertebrale secundum latius quam longum, hexagonum sequentium ninimum, tertio fere latius, tertiam nounihil longius quam latum, praeterea regulariter hexagonum, quarto multo angustius; quartum maximum, hexagonum, pone medietatem suam admodum dilatatum; quintum pentagonum, omnium, quoad partem suam posteriorem, latissimum, cum carinae acumine humili, obtusato; sextum campaniforme, quin to multo angustius, cum carina prima medietate angusta, acuta, altera lata, rotundata, recta; scutellum laterale primunı reliquorum minimum, parvum, circuli partem circiter quartam formans, margine externo subangulosum; secundum aequali latitudine, altissimum, margine superiore trigono, margine anteriore laterali inferiore arcuato, tertium secundo latius, quarti latitudine et forma, hoc tamen altius ac margine superiore trigono minus acuminatum, margine inferiore leviter arcuatum, lateribus, sicut secundum, rectissimum; quintun quarto media parte minus, subrhombeum; bracteae marginales viginti sex, prima trigona, tertia et penultinıa pentagona, reliquae quadrangulares, subrhombeae; sexta, septima et octava omnium latissimae; bractea utrinque ultima fere pentagona, pone acuninata; sternum cum thorace per synchondrosin conjunctum annulus oblungo-ovatus, angustus, utrinque e medio duo ossa, oblique ascendentia, cum thorace se jungentia, apice dilatata, recta, interstitium latum, quadrangulare inter se relinquentia, emittens, medio tegmine cartilagineo expletus; scutella disci duodecim, laevissima; duo 
printa, onnium minima, trigona, magine anteriore arcuato, sequentia duo, antecedentibus tertia parte maiora quadrangularia, tertia lata, subpentagona, fuarta, onnium maxima, multo latiora quam alta, tetragona, quinta, tertiis parmm minora, quadrangularia, latere externo ad pedes sinuata, sexta oblongo-trigona, margine externo subarcuato, subparia; supra juncturam sterni cum thorace scutella uninque quatuor tetragona, quormu ultimum latere inferiore angustatum; caput supra et ad latera scutellis corneis polygonis, supra fronten convexiusculis, vertex scuto maximo, plano polygono, pone recto-truncato tectum; scuta superciliaria utrinque tria, polygona, ultimum onnimm maximum, magnum cum margine cxterno crasso, rotundato, glaberrimo supra angulum oculorum posteriorem multo prostans; scuta pilei septendecim, temporum duodecin; maxillae inferioris latera scutellata; pedes pinniformes, toto scutellati, anteriores posterioribus apice rotundatis duplo longiores, supra et infra digitos et margine postico antibrachii scutis mignis, planis, latere superiore ac inferiore scutellis minoribus, polygonis, planis tecti; pollex et digitns index pedum tam anticorum quam posticormm ungniculati; unguis depresso-trigonus, validus, nomnilil introrsum flexus; ca uda brevissima, depresso-conica, scutellis planis aucta; auus fere infra caudae apicem.

\section{Animal adulıum. Testa suborbiculari.}

Color. Capitis, colli, pedum caudae ac totius testae pars superior castaneo-fusca, testa in rubrum nounilil vergens, scutis margine nigro- ac fulvo-radiatis; corporis pars tota inferior laete vitellino flava; irides obscurac(?); ungues corneo-fusci margine pallido.

Proportio corporis partium e mensura. Longitudo (speciminis junioris) a rostri apice ad

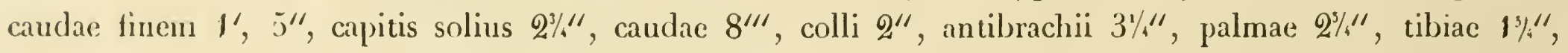
plantae 1\%", lloracis 93.", sterni 7", $7 "$, ambitus occipitis $6 \%_{2}^{\prime \prime}$, testae immediate pone antipedes $18^{\prime \prime}$, in medio 16\%", ante femona 16\%"' ; altitudo testae in medio 4", 8"'; eiusdem latitudo in medio 8", sterni 7"'; oculi distant a naribus 5"', ab auribus 7"'; latitudo capitis supra oculos $1^{\prime \prime}$, 8"', pone naves $8 \%$ '".

Habitatio et vilae ralio. Habitat sub zona torrida; vidi specimina in mare littora Brasiliae et Hispaniae alluente, capta; specimen maximum, 3', 7" longum, ex mare rubro descripsit cl. Bruce; caro saporis ingrati.

OBSERVATIONES. Speciem hanc habet Museum Parisiense ac Vienense; duo specimina possidet amicissimus Dr. Agassiz, qui maioris picturam ad delineandum in lapidem mecum communicavit. - A simili Chelonia Caounna differt animal junius scutellorum dorsi pictura ac crassitie fortiore, aetate provectius praeter dorsi picturam testa sina suborbiculari. 



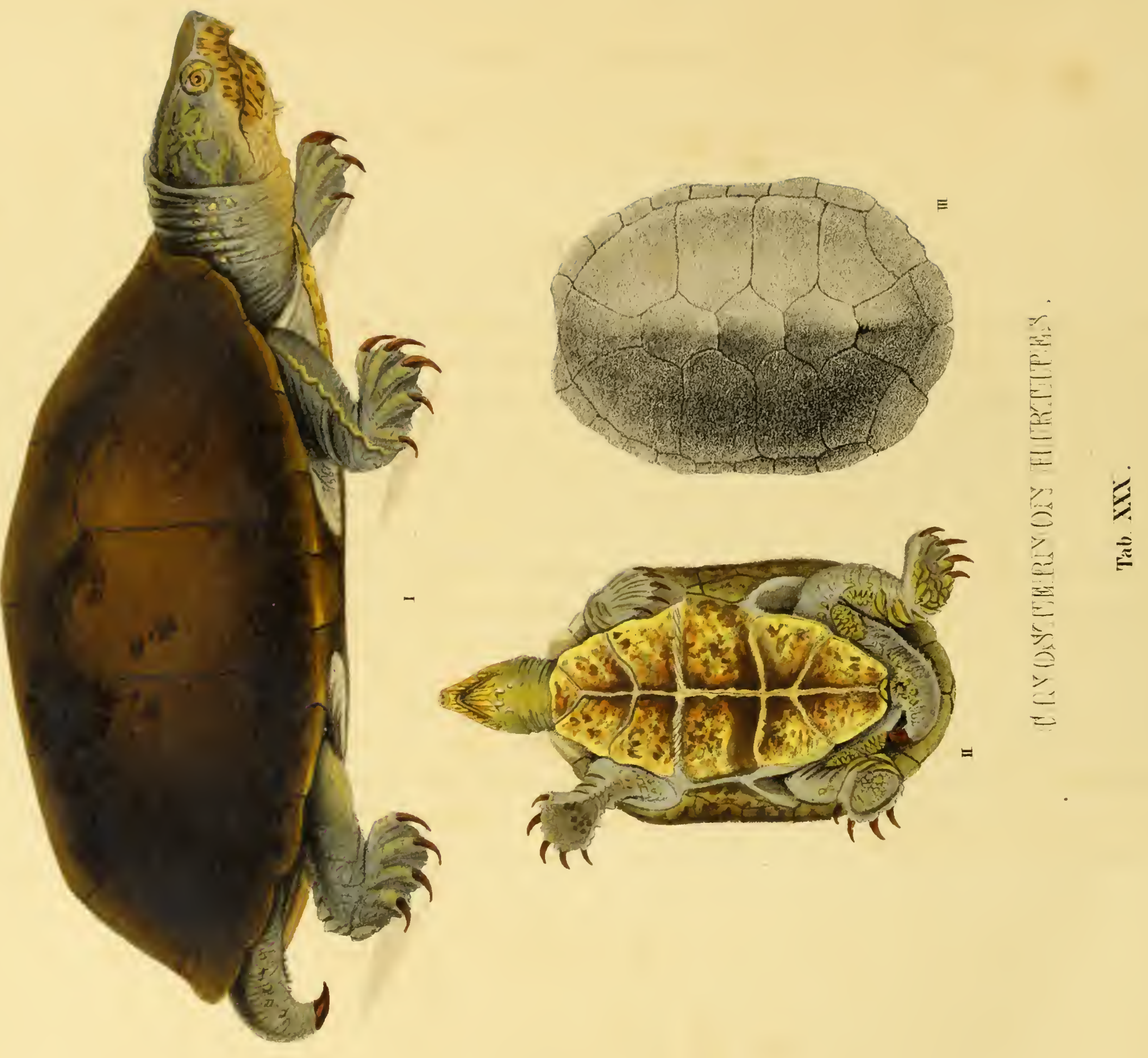




\title{
CINOSTERNON HIRTIPES.
}

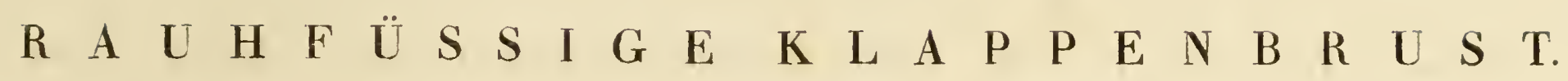

\author{
Т А в. XXX. Fig. 1, 2, 3.
}

Character essentialis. Testa ovata, glabra, convexa, supra spinam planiuscula pone pelvin compresso-elevata, olivacea; sterno oblongo-ovali fusco-et flavido-varioloso; cauda tota collo pedibusque supra cinerascenti-olivaceis; mento bicirrhoso flavido; tibiis femoribusque latere postico scutello ovato echinato auctis; pileo et rhamphotheca variegatis.

Synonmia. Cinosternon hirtipes Wagl. Natürl. Syst. d. Amph. Tab. V. Fig. 29.30.

\section{E S C R I P T I O.}

Corporis forma universalis. Caput depressiusculo-ovatum, ad tempora convexum, ante oculos attenuato-compressiusculum supra cute communi glaberrima tectum; rostrum supra maxillae apicem multo productum, anterius deorsum et introrsum truncatum, rotundato-compressum; maxilla mandibulam pyxidum instar includens, tomiis utrinque ad latus apicis integri profunde uni-sinuata; gnathotheca maxillae in paratonis convexiusculo-plana, mandibulae paullo convexior; maxilla gonyde lata, percuam ascendente, convexa, glaberrima, in apicem simplicem, ore clauso foveam ossis intermaxillaris intrans terminata; tomia mandibulae versus eius apicem arcuato-ascendentes, integerrima angulus mentalis trigono-rotundatus, papillis duabus pendulis, teretibus auctum; rhinarium in apice extimo rostri, subcirculare, convexo-prominulum, naribus rotundis, mediocribus, simplicibus; oculi modice magni, subobliqui in angulo rlinothecae, naso quam auribus propius siti; palpebrae superioris rudimentum uniplicatum in orbitae angulo anteriore oblique expansum; palpebra inferior magis angulum orbitae posteriorem inferiorem occupans completa oculım totum in linea obliqua obtegens; pupilla rotunda (?); aures cute communi obtectae supra et pone oris angulum sitae; collum cum capite sub testam retractile modice longum, validun, cute laxa induta, rugis linearibus, subtilibus notata, glabra, attamen supra verrucis erectis, conicis, parvis, per lineas circiter 9 longitudinales (quarum duae intermediae et extimae reliquis minus distinctae), dispositae.

Testa ovata, postice parum angustior quam antice, convexa, supra humeros applanato-depressiuscula supra pelvin convexior, et paullo pone hanc elevato-compressa; margo ad latera testae angustissimus et huius fine postica recto descendens, reliqua testae parte oblique prominulus, angustus; scutella disci integerrima, glabra, tredecim, i. e. vertebralia quinque, lateralia utrinque quatuor; illa his multo minora; scutum vertebrale primum calyciforme, sub sequente multo angustius; hoc margine anteriore arcuatum, utrinque in medio laterum in angulum acutum excurrens, pone recto-truncatum, unde biangulosum; scuta duo sequentia forma nunc descripti sed margine anteriore recto-truncata horum posterius apicis postici medio compresso-giblum; scutum vertebrale ultimum, quintum, distincte quadrangulare, parte postica dilatatum medio carinata compressum, tertium scutorum vertebralium latissimum; scutellum laterale utrinque primum, subrhomboidale; secundum et tertium nunc descripto angustiora pentagona, multo altiora quam lata, supra subacuminata, ambo magnitudine aequali; scutellum quartum, ultimum utrinque, pentagona, angulo postico inferiore oblique truncato; bracteae marginales viginti tres, subnodoso-planae, integrae; prima harum minima, infra testae marginem solummodo conspicua; sequens utrinque trigona, omnes reliquae oblongo-quadrangulae, exclusa utrinque bractea penultima, omnium altissima, quadrangula - triangula, margine superiore oblique ad angulum posticum inferiorem scutelli disci

Wigler, Amphib. III. 
lateralis ultimi sursun ducto; bracteae ulimae praeter nunc descriptam altissimae; sternnm cum thorace per symphysin intime utrinque conjunctum oblougo-ovale, thorace hrevius, planum, margine integrum, loho anteriore et posteriore molite, scutellis undecim tectum, in medio marginalis apicalis lobi postici parmm cmargiuatum; scutellum anterins medium parvun trigonum, margine anteriore rotundatum, sequens utrinque ad latera subrhomboülale, secundum quadrangulare, latere externo dilatatum; pone hoc lobus sterni cum lnius parte intermedia ligamentis nohiliter connexus; scutellum utrinque inter medimm, i. e. laterale tertimn quadrangulare, parte anteriore quam postica latius, scutellorum sterni omnium maximum, pone cum lobo sterni postico aequo tuodo ut cum eius anteriore connexum; scutellum quartun quadrangulare, latere interno coarctatum, sextum, i. e. ultimum trigonum, margine externo rotundatum; junctura sterni cum thorace longula, scutello subelliptica obtecti.

Pedes modice longi, robusti, depressi, maxima ex parte cute nuda, glabra, parum rugulosa tecti; squanace corneare quatuor lineari-arcuatae, niargine liberae supra maniculorum basin, versus huius latus internum, tales quiuque fortiores, magis prö̈minentes, infra marginem asperulae supra podariorum calcem; limbus cutaneus, subfimbriatus nargine externo antipedum; huius loco in antipedum margine externo compresso series papillarum brevium conicarum; papillac tales in pagina posteriore femorum per series longitudinales 4 5, codem loco in antipedibus per series circiter 3 dispositae: clypeus ovatus in fenornm parte posteriore inferiore et alter talis eodem loco in tibia, illi femorum oppositus, duriusculis, tota verrucis conicis, duriusculis echinatus: inembrana natatoria inter digitos admodum lata et laxa, margine anteriore subfimbriata: plantae naniculorum et podariorum verruculosae et rugulosae: digiti lorum quatuor, illorum quinque unguiculati: digitus externus in podariis incrmis: ungues validi, perquan arcuati, acutissimi, tereti-compressiusculi, tam in maniculis quam in podariis aequali fere magniturline, unguis tamen digiti externi reliquis minor: cauda ad latus dextrum sub testae marginem flectenda, validissima, praesertin hasi, conica, apice ungue valido conico-inflexo exstructa, subus, medio longitudinaliter subimpressa, circa anum papillis conicis brevilous ac talibus supra per series longitudinales dispositis obsita, cacterum nuda, rugulosa: an us a caudae basi subremotus rotundus, anmbitu rugoso.

Color. Caput totum olivaceum lituris marmoreis dilutioribus, rhamphotheca flavida maculis fuscescentibus variegata: cauda tota, collum et pedes supra cinerascenti-olivacea, immaculata, subtus albido-flavida: testa tota olivacea: scutella singula maculis obscurioribus vix conspicuis nubilata: sternum flavidum, fusco-rariolosum: ungues et uncus caudae obscure corneo-fusci: oculi ....?

Proportio corporis parlium e mensura. Longitudo a rostri apice ad caudae finem 8",

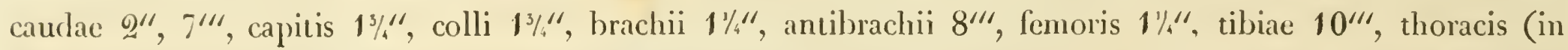
linea recta) $4^{\prime \prime} 3^{\prime \prime}$, sterni $3^{3}, "$ : ambitus occipitis $3^{\prime \prime}$, $5^{\prime \prime \prime}$, testae immediate pone antipedes et in medio $8^{\prime \prime}$, supra pelvin 7/2": latitudo sterni immediate pone antipedes $2{ }^{1} 3^{\prime \prime \prime}$, ante scelides $1 ", 10^{\prime \prime \prime}$ : oculi distant a rostri apice $4 \%{ }^{\prime \prime}$.

Habitatio et vitae ratio. Habitat in Mexico.

OBSERVATIONES. Specimen purissimae conservalionis recentissime necalum in spiritu vini conditum, hic descriplum el depictum misil Mexico ad Museum Monacense dom. baro DE Kantrinskr. Post anni decursum colorum mulalionem in hac teshudine non observavi. - HERNANDEZ eam non habet. 



$$
8
$$




\section{PERIOPS HIPPOCREPIS.}

H U F E I E N F L E K I GES S G H I L D E R A U G.

\section{T а в, XXXI.}

Character essentialis. Notaeo nigro catena e lineis albo-flavidis regulari supra spinam dorsi et caudae extensa ornato, utrinque ad latera cum lineis concoloribus, figuras rhombeas formantibus colaerente; gastraeo albido-flavido utrinque ad latus maculis angulosis atris notato; occipite fascia atra obsito formam ferri aequini aemulante, ab altera frontis fasciola albida diremta; temporibus, cervicis collique lateribus aurantiacis. Scut. abd. 232 - 235, sculel. subcaud. 79 - 82.

Synonyin. Coluber hippocrep is Linn. Mus. Ad.Fr.1.p.16. f. 2. Gmel. Linn. Syst. Nat.T.3. p. 1117. Lacé P. Hist. nat. des serp. II. p. 117. Uebers. v. Bechstein 4. p. 120. t. 15. f. 1. (fig. Linv.) Latr. Hist. nat. des. rept. IV. p. 130. Daud. Hist. nat. des rept. VI. p. 249. MIE r r. Nat. Syst. Amph. p. 105. n. 50. ${ }^{*}$ Natrix hippocrepis Laur. Rept. p. 77. - Couleuvre... Geoffr. Descript. de l'Égypte tab. 4. f. 3. Natrix bahiensis Wagl. et Six. Serp. Brasil. t. 10. f. 2 (icon. e pullo, decolorato).

\section{E S C R I P T I O.}

Corporis forma universalis. Caput subdistinctum, oblongo-ovatum, depressum, ad latera oculorum et in pileo planum; rostrum longulum, latiusculum, obtusum, subdepressum, lateribus planum, cantho subdistincto; maxilla quam mandibula paullo longior; oris rictus rectus, versus angulum subadscendens; nares immediate infra canthum rostralen in margine scutellorum duorum sitae, maiusculae, rotundatae, simplices; oculi maiusculi subcirculares, subdetrusi, pupilla circulari; scu ta capitis; scutum rostrale latum trigono-rotundatum, parum convexum; scuta frontalia anteriora duo posterioribus parum minora, magna, obsolete pentagona, pone oblique truncata, posteriora duo distinctius pentagona, latiora quam longa, cum latere externo supra canthum rostralem deflexa; scutum verticale mediocre distincte campaniforme, planum; scuta superciliora anguloso-ovata, antice angustata, supra convexiuscula; scuta occipitalia duo mediocria, pentagona, antice dilatata, postice in apice recto-truncata, supra plana; scuta temporalia nulla, horum loco squamae laevissimae polygonae; scuta nasalia tria, anterius, omnium maximum, quadrangulum, posterius pentagonum; maiusculum, tertium infra nares situm, minimum, rhombeum; scutum loreum unicım, masculum, quadrangulum; oculus scutellis septem cinctus, excluso scuto superciliari; scutella duo anteriora, horum maxima et tria inferiora (horum ultimum praecedentibus duobus multo maius) (quinquangula, posteriora duo angustissima, subquadrangula; scuta marginalia labii superioris utrinque novem, anteriora sex parva, quadrangula, septimum et octarum maxima, hoc quadrangulum, illud pentagonum, ultimum trigonum, postice acuminatum; scuta marginalia labii inferioris novem, anteriora quatuor coarctata, quintum et sextum omnium maxima, magna, hoc penta-, illud tetragonum, reliqua magnitudine sensim decrescentia; quadrangula; scutum labiale parvum trigonum; scutorum labialium paria duo, horum anteriora oblongo-rhonıboidea, posteriora anterioribus multo longiora et angustiora; squamae gulares planae, strictae, oblongo-sexangulae; dentes maxillares utrinque quatuordecim, reclinati, posteriores sensim maiores, omnes solidi, palatini his conformes, minores, multo numerosiores, primores nulli, mandibulares utrinque quindecim (?) reclinati, posteriores sensim minorem

*) Coluber domesticus Livaei a Merremo cum Per. hippocr. auctus, nobis e Graecia missus, differt toto coelo et spectat genus peculiare.

WAGLER, AmpHib. III. 
attanch quoque 3-/4 anteriores sulsequentibus minores; truncus longissimus, gracilior, teres, gastraeo planiusculo subbianguloso; scuta abdominalia lata, brevia, margine posteriore rectissino; squamae notaci numerosissimac, parvac, laeves, longac, subellipticae, laterales sensin maiores, inbricatac; cánda mnediocris, teres supra sfuamis hexagonis, planiuscula subus, scutellis per duplicem seriem sexangnlis tectal, sensin in acumen desincns. Scuta abdominalia 232 - 235 scutella subcaudalia uni us seriei $79-82$.

Color. (Animal adultum.) Notaeum totum atrum; catená e lineis albido-flavidis formata distinctissima, adnodum regularis supra spinam dorsi et cauda, utrinque latere externo cum lineis concoloribus, figuras rhombeas formantibus, ad latera caudac indistinctis, cohaerens; macula nigra forman ferri equini acmulans in occipite, cum utroque apice infra oris angulum desinens, postice in neclio in maculan supra cervicem ovatam magnam excurrens; macula gemina parva oblongo-ovata, medio nigra, in scutis occipitalibus; fascia nigra frontis inter oculos, a macula occipitali linea transversa albida (medio alıcra linea strictiore, pone oculum versus angulnum oris excurrente nigra, dissecta) diremta; scuta nasalia nigra, albo-trifasciolata; maculae tres nigrae ad latera labiorum maxillae, haec ipsa sicut latera capitis, cervicis et colli aurantiaca; color ille aurantiacus post collum sensinn pallidior; gastracum flavido-album, utringue ad latus maculis maiusculis angulosis nigris variegatum; cauda subtus flavido-albo et nigro variolosa scutum anale ad aurantium nonnihil inclinans; irides. . . ?

Animal pullum et juvenile. Nuchae, occipitis collique latera albida, animalibus aetate provectioribus sensim in isabellimum vergentia; maculae dorsi nigrae olivaceo-brunnescentes, angustius nigromarginatac; scutum anale et cauda subtus interdum aurantio-lavata.

Proportio corporis parlium e mensura. Longitudo a rostri apice ad caudae finem $3^{\prime}$, $4 \% \%^{\prime \prime}$, caudae $6 \%{ }^{3 \prime}$, capitis $1^{\prime \prime}, 3^{\prime \prime \prime}$; oculi distant a rostri apice $4 \%_{2}^{\prime \prime \prime}$.

Habitatio et vilae ratio. Habitat in Aegypto, Barbaria, Gracia, Sardinia, Hispania.

OBSERVATIONES. Vidi specimina e locis supra indicatis. Specimen quod depingi curavi, nitidissimum, cel. Dr. Rud. W animale morluzo mox pallescit. Periops hippocrepis servatur in Museo Parisiensi, Berolinensi, Viennensi et Lugdunensi. 



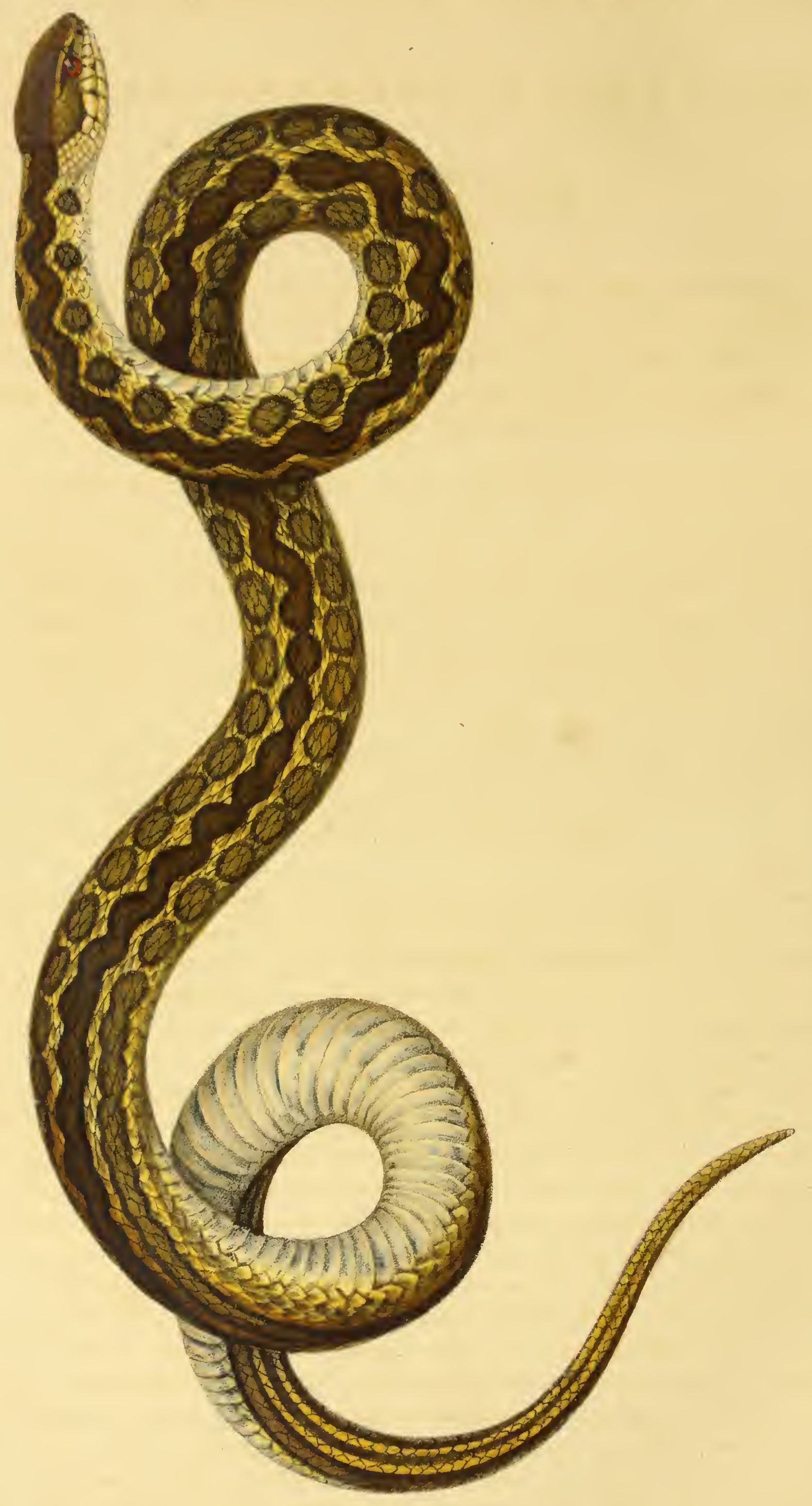




\title{
COELOPELTIS RHOMBEATA.
}

\author{
R A U T E N F L E G K I G E G R U B E N S G H L A N G E.
}

\section{T A в. XXXII.}

Character essentialis. Notaeo dilute olivaceo supra spinam dorsi taenia fusca, nigro-marginata, undulata, in cauda recta, ad latera utrinque serie macularum subrhomboidaliun, fuscarum, nigro-marginatarum, versus caudam et in hac in taeniam confluentibus notato; pileo fusco; occipite fascia obscura circumdato; gastraeo albido, maculis in scutorum margine posteriore nigrescentibus, pusillis variegato. Scut. abd. $164-170$, scutel. subcaud. $65-70$.

Symonymi a. Scheuchz. Physic. Sacr. tab. 739, f. 1. Seba Thes. I. t. 19. f. 7. II. t. 11. f. 3 t. 15. f. 2. t. 38. f. 1. Coluber rhombeatus Linv. Mus. Ad. Frid. p. 27. t. 24. f. 2. (Descriptio e specilmine sine epidermide) Grel. Linv. Syst. Nat. 1. 3. p. 1099. n.227; (Figura cita ta 2 in Scheuchzeri tabula 746 spectat Linnaei Colub. miliare m* ${ }^{*}$ Daubent. Encycl.. meth., Lacép. Hist. nat. des serp. 1. p. 412. Latr. hist. nat. des rept. IV. p. 150. Daud. hist. nat. des rept. VII. p. 119. Merr. Syst. d. Amph. p. 111. n. 72. - Coluber tigrinus Linn. Gmer. Syst. Nat. p. 1113 et reliquor. auctor. (Sebae.

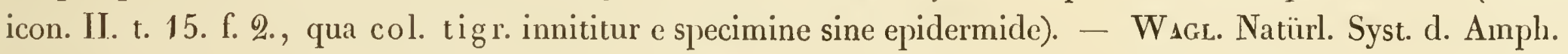
p. 189.

\section{E S C R I P T I O.}

Corporis forma universalis. Caput subdistinctum, fere aeque altum ac latum tetragonoovatum, pileo plano, lateribus occipitis rotundatis; rostrum breviusculum, supra versus apicem subdeclive, altum, lateribus impressiusculum, acutiusculum, cantho subdistincto; oris rictus versus angulum posteriorem arcuato-subascendens; nares in margine scutellorum duorum sitae, laterales, mediocres, simplices rotundae; oculi laterales, mediocres, parum obliqui, subcirculares in medio capitis siti, pupilla circulari; lingua tota nigricans; scuta capitis: scutum rostrale pentagonum, apice postico acuto sursum et retro flexum, margine inferiore sinuato-impressum; scuta frontalia anteriora duo mediocria: subtriangula, postice dilatata, posteriora maiora pentagona; scutum verticale elongatum, medio impressiusculum, campaniforme, antice recto-truncatum; scuta occipitalia duo scuto verticali parum latiora, subtriangula, margine externo obliquo; scuta superciliaria longa, plana, scuto verticali parum breviora, postice recto-truncata, antico attenuata, margine externo supra oculos prosiliente; scuta nasalia duo mediocria, anterius rhomboïdale posterius pentagonum; scutum loreum unicum, quadrangulum, infra dilatatum; scutum oculare anterius infra attenuatum, supra dilatatum, margine superiore prosiliente, cum margine scuti superciliaris cohaerente; scuta ocularia posteriora duo, quadrangula, magnitudine aequali: scuta marginalia labii superioris utrinque octo, posteriora magnitudine sensim crescentia, primum minutum et sicut sequentia duo, obliquum, quadrangulum, quartum et quintum subquadrangulum, sextum quadrangulum infra dilatatum, septimum, onnium maximum, et octavum pentagonum: scuta marginalia labii inferioris utrinque decem, anteriora quinque coaractata, obliqua, horum primum omınium longissimum cum apice apicem huius scuti alterius attingens, sextum omnium maximum, magnum, pone acuminatum, scuta reliqua rhombea, posteriora magnitudine sensim decrescentia: scutum labiale parvum trigonum: scutorum mentalium paria duo: scuta oblonga, anteriora postice oblique truncata, angusta, posteriora anterioribus parum breviora postice subacuminata: scuta temporalia nulla; squamae duae maiores supra scutum labiale sextum et septimum maxillae: squamae gulares laevissimae, planae, mediocres, imbricatae: dentes 
primores nulli, maxillares utringue duodecin retroversi, validi, qosteriores sensim maiores, tres ultimi rongati, recti, retroversi, gingiva laxiore inclusi, sed non perforati, dentes mandibulares nediocres utrinque duodecim suberecti, posteriores magnitudiue seusin decrescentes: palatini mixillarimm forma, numerosi truncus elongatus, rohusus, teres: squamac notaci totius lanceolatac, medio impressiusculac: unde subconcavac, longae, imbricato-positae, penulhimac et ultimac seriei subrhombö̈dales, planae: scuta abdominalia lata, glabra: canda teres, sensim in acmmen acutiusculnm desinens, breviuscula, subtus serie duplici scutellormu sexangulorum. Scuta abdominalia 16/,-170, scutella infra caudalia unius seriei $65-70$.

Color. Pileus olivaceus, unicolor; notaeum totum dilute olivaceum: taenia postice ex medio maculace arcuatae occiput cingentis fiscae excurrens undulata, ex parte unaculis subroundis sive rhombeis forı1ata, fusca, niggro-marginata supra spiuam dorsi et caudac ducta, attamen supra hanc iu utrogue uraargine integerrima: taenia altera talis simili modo tincta, in utroque notaei latere, e maculis rhombeis, partim separatis, partim inter se connexis composita, versus trunci partem posteriorem et in candae lateribus margine utroque integerrima: gastracum totum abidum: scuta abdominis ad margincm maculis pusillis nigrescentilus variegata: cauda subtus unicolor: scuta margiualia labiorum flavida, nigrescenti-subnubilata: irides. . . ?

Proportio corporis partium e mensura. Longitudo a rostri apice ad caudac finem 3/4", caudac 7", capilis 1"; oculi distant a rostri apice $3^{\prime \prime}$.

Habilutio et vitae ratio. Habitat satis frequens ad Promontorium bonae spei.

OBSERVATIONES. Hanc serpentem habet museum Parisiense, Berolinense, Lugdunense, Viennense. Specimen nitidissimum adest in mea Amphibiorum collectione. Epidermide detrila pileus in album, dorsum in griseum, eius maculae in nigrum vergunt. In speciminibus in spiritu vini conditis color naturalis non facile evanescit. 


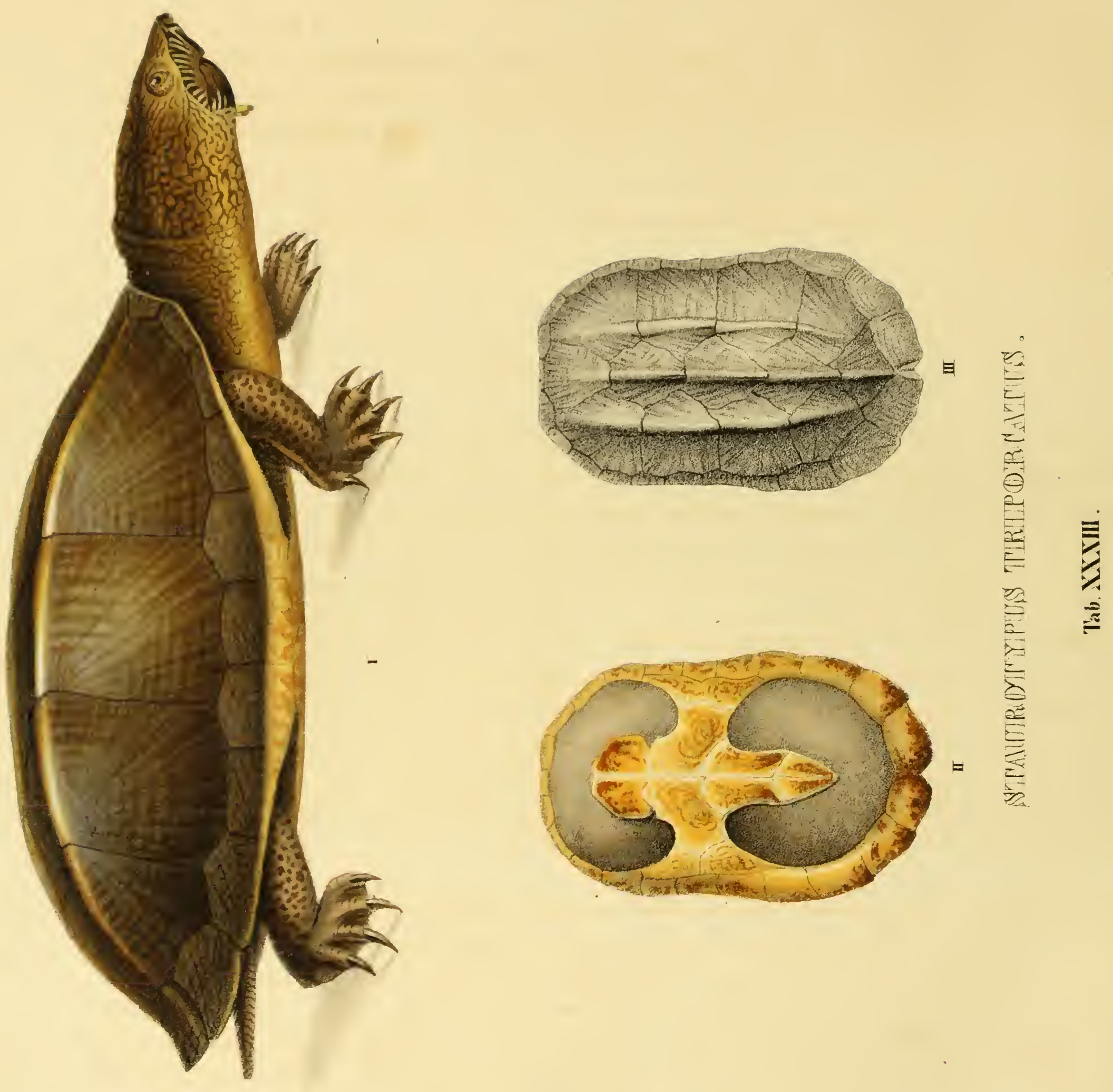


\title{
STAUROTYPUS TRIPORCATUS.
}

\author{
$\begin{array}{lllllllllllllllllllllll}\text { D } & \text { R } & \text { E } & \text { I } & \text { K } & \text { I } & \text { E } & \text { L } & \text { I } & G & \text { E } & \text { K } & \text { R } & \text { E } & \text { U } & \text { T } & \text { Z } & \text { E } & \text { M } & \text { Y } & \text { D } & \text { E. }\end{array}$
}

T А в. XXXIII.

Character essentialis. Testa oblonga, convexa, antice truncato-retusa, lateribus intracta, postice rotundata, margine horizonlali-devexo dilatata, supra fusca, tricarinata, carina intermedia valde elevala, pone cultrala; lesta subtus el sterno flavidis; capite el collo supra reticulato-variegalis.

Symonyma. Testudo pensylvanica Var. Shatv. Gen. Zool. Vol. II. p. 61. t. 15 (testa). Terrapene triporcata WiegianN, Jsis 1828. p. 364. - WAGL. Natürl. Syst. d. Amph. p. 137. tab. V. Fig. 44. 45.

\section{E S C R I P T I O.}

Corporis forma universalis. Caput cute communi glabra tectum, ovato-trigonum, latius paullo quam altum, occipitis lateribus rotundatum, fronte plana, rostro admodum porrecto, acutiusculocompresso, introrsum obliquo-truncato, nıxillae apicem multo transcedente; mandibula quam maxilla multo brevior gnathidiis versus apicem arcuato-adscendentibus, paratonis latiusculis convexis; tomia maxillae tonia nıandibulae includentia acuta, ante apicem utrinque uni-sinuata, gnathotheca glabra alta, convexa, margine superiore undulata, versus oris angulum magnitudine sensim decrescens, in eodem acuminato-angustata; mandibulae apex ascendens ore clauso fovean ossis intermaxillaris intrans: gonys trigona, lata, pone rectiusculo-truncata supra convexa; augulus mentalis angustiusculus, arcuatus, papillis duabus cutaneis pendulis obsitus; rhinarium in apice extimo rostri situm, rotundatum, convexum, prominulum, naribus lateralibus, rotundato-oblongis, patulis, simplicibus; oculi mediocres rostro paullo propiores quam oris angulo; a ures cute communi prorsus obtectae; collum cum capite sub testam retractile, longulum, validum, cute laxa indutum, rugis linearibus, annularibus, subtilibus supra cervicem et nucham reticularibus dissecta, unde locis ultimo dictis asperula.

Testa oblonga, convexa, antice truncata, postice rotundata, lateribus subintractis; margo ad latera et praesertim in apice antico angustissimus, devexus, in apice postico latiusculus, horizontali-devexiusculus, medio subsinuatus; reliqua marginis pars testae integra, haec supra in medio carinis tribus, longitudinalibus admodum distinctis, rectissimis aucta; culmen carinarum rotundatum, carinae intermediae valde elevatum versus apicem posticum cultratum; scutella disci tredecim, i. e. vertebralia quinque, lateralia utrinque quatuor; scutum vertebrale primum subquinquangulum, antice attenuatum, ad latera a medio ad apicem posticum margine obliquo, ibidem supra in medio compresso-elevatum; sequens oblongum, subsexangulum, in utroque apice, praesertim in postico subattenuatum, supra in medio (sicut scutella tria subsequentia) carina elevata, longitudinali (in scutello ultimo cultrata) auctum; scutellum tertium secundo paullo maius subquinquangulum, antice truncatum, postice acuminatum, quartum subrhomboïdale, marginibus obliquis rectis, apice antico subfurcatum, postico lanceolatoacuminatum, quintum scutello praecedenti simile sed latius, postice minus acuminatum; scutellum laterale primum, secundum et tertium magnitudine aequalia, supra ante medium carina longitudinali, elevata, in scutello quarto conspicua, aucta; primum quinquangulum, margine externo subundulato arcuatum, interno in angulum ex medio excurrens, secundum margine laterali et externo rectiusculum, interno in angulum ex medio excurrens, tertium secundo forma simile, attamen apice postico externo subtruncatum, quartum, scutellorum lateralium; magnitudine inter se subaequalium, minimum, pentagonum, 
apice postico externo obliquo tmucatmm; bracteac marginales viginti tres, omnes, exclusis tribus ultunis ntrinsque lateris, angustae, oblomga-quadratae, magniundine inter se subaequales, prima media tenuis, lathuscula, mangine postico subangulosa, sepuens utrinque in medio marginis postici in angulum brevem excurens, nonat magine externo recta, interno ad apicem anterinem oblique truncata, decima ot mudecima quadraugnla, haec antice, illa postice dilatata; superficies scutellormm omnimm testae ghabra: sternum toto osseum, minutum, thorace triplo et nlta minus, cum thorace per symphysin connatum, cruciforme, prorsus planum, glabrum; lobus sterni anticns mobilis, hiscutatus, postico latior, hevior, cum stemi parte intermedia liganento flexibili lobus posticus cum eodem per symphysin comexus, immobilis lanceolatus, latcribus arcuato-emaginatus, triscutatus; stemi pars media subarcuata, in ntroque margine multisinuata; scutellum sterni utrinque primum subquinquangulum, antice apice exterus oblique truncatum, margine postico arcuato-simuatum; scutellum utringue subsequens margine intice Iri-postico bisinuatum, obliqnum, terlium margine antico arcuatum, postico oblique truncatum, externo arcuato-simutum; scutellum extimun impar (?) cortiforme, sed antice in utroque latere oblique truncitum; junctura sterni latinscula in utroque appec oblique truncata, ntrinque scutellis duabus juadrangularibus, glabris tectil.

Pedes Ginosterni, nudi, exclusis scutellis linearibus, transversis, margine postico liberis in latere postico tibiarum; antibrachium latere externo plica longitudinali cutanea auctum; digiti modice longi, ommes membrana latissima ultu unguium basin extensa, laxa, margine antico subcrenulata palmati, omnes mugniculati, excepto digito externo podariorum; ungues longi, rectiusculi, acutissimi; caudá brevis, conica, squamis minimis nonnullis erectis munita, unde asperula.

Color. Testa supra dilute fusca, obsolete obscurius striata, fere unicolor, margine inferiore cum sterno tlara; hoc fusco subvariolosum; caput, collum et pedes fusca, illa supra uigro-reticulata; gnathotheca llavida, striis punclisque corneo-fuscis belle variegata; nngues corneo-fuscescentes; irides ....?

Proportio corporis parlium e mensura. Longitudo testae (supra eins convexitatem) 123/", cius latitudo (supra convexitatem) $10 \%$ ".

Habilatio et vitae ratio. Habitat in Mexico, in flumine Alraredo.

OBSERVA'TIONES. Specimen quod descripsi effarchum, optime conservatum, misil dom. DEPPE ad Museum Berolinense; iconem ex eodem mecum communicavit dom. Dr. et Prof. MrEgMANA.

*) In medio linea longitudinali subexarata, sed non prorsus divisa. 



$$
50
$$




\title{
GERRHOSAURUS FLAVIGULARIS wIEg.
}

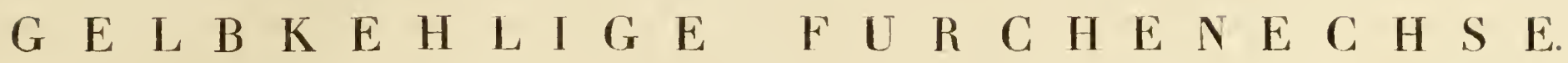

\section{T н в. XXXIV. Fig. 1.}

Character essentialis. Notaeo fusco-olivaceo, linea sulphurea, pone oculos incipiente, nigro marginata in utroque latere, mento gulaque et scutis marginalibus labii superioris laete vitellinis, pectore et abrlomine albidis, coeruleo pallide lavatis, cauda basi tetragona, versus medium tereti, corpore sesquilongiori, poris femoralibus utrinque tredecim.

Synonyma. Gerrhosaurus flavigularis. Wiegmann Isis XXI. fasc. III et IV. p. 379. Wagl. Natürl. Syst. d. Amph. p. 158. - Lacerta Seps Lin. S. N. XII Nro. 17. p. 363?

Seps Shaw General Zool. III. p. 252.

Scincus sepiformis. Schneid. Hist. amphib. II. p. 191. Merr. Syst. Amph. p. 70.

Tachydromus Seps Merr. S. A. p. 69.

\section{E S C R I P T I O.}

Corporis forma universalis. Caput cum trunco continuum, tetragono-pyramidale, a vertice sensim declinatum, ante aures intumescens, in apice obtusum, undique scutellis multangulis irregularibus vestitum; nares in rostri apice, laterales; oculi laterales in medio capitis siti, palpebris duabus squamulosis tecti; aures apertae, ovales, scuto semilunari flavo in margine antico munitae, subtus plicae lateralis initio nonnihil absconditae, ut tanquam foramen triangulum extus appareant, tympanum in meatu auditorio brevi depressum. Scutum rostrale semicirculare; scuta marginalia labii superioris 5 , primum trapezoïdeum, secundum, tertium subaequalia, quadrangula, quartum illis multo longius, subquadrangulum, quintum elongato-trigonum, apice meatus auditorii scutellum attingens; scuta labii inferioris quatuor, tertium ceteris maius; scuta nasalia duo parva, alte trapezoïdea, in confinio naribus pertusa; scutum loreum subquadratum, orbitale anterius polygonum, utrumque magnum, hoc pone scutello parvo, perangusto, subquadrangulo, inter scutella labialia et prima suborhitalia sito, auctum; scutum frontale anterius latum elongato-rhombeun, angulis lateralibus truncatis $(O)$ hexagonum; frontalia posteriora irregularia, subtrapezoïdea; verticale elongato-hexagonum, latum; scutella superciliaria 4, duo intermedia maxima, irregularia, subquadrangula, externa parva, triangula; scutella supraorbitalia 5 anticum parvum, trapezoïdeum, secundum angustum elongatum, pentagonum, posteriora tria minora, quadrangula; scuta postorbitalia 3 parva, quadrangula; orbitam infra cingunt scutella 8 parva, angusta, quorum anteriora ceteris longiora; scuta occipitis 5, intermedium parvum elongate-rhombeum, quatuor magnis undique inclusum, quorum anteriora duo obliqua, lata, trapezoïdea, posteriora duo maxima, irregulariter pentagona; scuta temporalia 5 intermedium parvum quadratum, quatuor permagnis, irregulariter multangulis inclusum; mentum scutellis quatuor tectum, quorum anteriora trapezoïdea, posteriora duo multo maiora, irregularia. Lingua basi crassa, lata, versus apicen obtusum, vix emarginatum sensim attenuata, ultra medium affixa. Dentes maxillarum numerosi, maxillae margini interno adnati, conici, subrecurvi, anteriores simplices, posteriores denticulo antico sub apice aucti, palatin i 8 simplices, obtuse conici.

Truncus continuus, cylindricus, lateribus intractis perpendicularibus subtetragonus dorsi fastigio rotundato obtusus, plica laterali utrinque ab oris angulo infra meatum anditorium in colli latere, et deinceps sub humero in pectoris abdominisque latere excurrente et prope femora sensim evanescenti insignis. Cervix et dorsum squamis adpressis quadrangulis, scutiformibus, per series transversas dispositis verticillata. 
Squanac nuchae et latermu plerumque quadratae, laeves; squanae dorsi acute carinatae, in spina dorsi pone angustatae, a lorma quadrata nomilit recelentes, cantuis singularnm in lineas mulas clevatas longitudinales snpra dorsun dnctas confluentibns. Gula squanis magnis latis hexagonis, ut in scincis, vestita; squamae pectoris maiores, forma irregulares. Abdo une ne sentis transverse seriatis vestitum. Scuta abdominalia angusta, in quavis serie octona, quorum intermedium trapezimm, chera rhomboidea, omnia carinis dnabus subobsoletis, parallellis, cum carinis suprà et infrà adiacentium in strias Iongitndinales conflucntibus instructi.

II umeri ce antibrachia squanis latis, hexagonis vel rotmudatis, laevibus; femora et crura intus squanis magnis, laevibus, plerumque hexagonis, extus minoribus rhombeis imbricatis tecta; palmae plan-

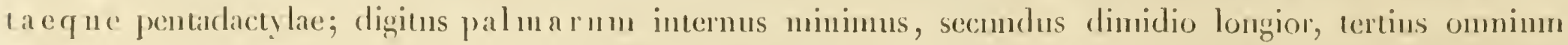
masimns, quartus tertio sublacenal is, quintus hoc dimidlo brevior; plan tae graciles, digitis valde inaequalibus, ab) interno minimo ad (prartum maximun longitudine crescentes, quinto remoto, vix quartum dimidium aequante. Femora poris perconspicnis, papillosis, subcylindricis tredecim instructa. A nus transversus, scutis phrithus magnis, irregulariter quadrangulis antice tectus. Cauda longissima, al basin lateribus perpendicnlaribus tetragona, versum medimn teres, sensim attenuata squanis quadrangulis carinatis, caudae appic'mn versus ungis magisque angustatis, verticillata, corpore sesquilongior. Squamae candae superiores láteribus se invicen tegentes, hinc subimbricatac, carina pone emergente mucronatae, striis pluribus versus carinan convergentibus notatae; inferiores praeter carinam laeves. Ungues falculares, compressi, acuti.

Color. Caput, collum, dorsum, latera candac superficies superna fusco-olivacea; vitta pone oculos incipiens sulphurea, nigro utrinque marginata, ad colli et trunci latera supra femora excurrit et in cauda media paullatim evanescit. Scuta labii superioris, mentum, gula et scutellum in meatus auditorii margine antice colore vitellino, laetissimo splendent; abdonen, pedum interna latera et cauda subtus colorem albnun, uomnihil cocrulescentem ostendunt.

Proportio corporis parlium e mensura. Longitudo a rostri apice ad caudae finem 10\%" mensurac Rhenarae; caudae solius $6 \%$ " trunci (al) apice rostri) ad anum usque $4 \%{ }^{\prime \prime}$; capitis $3 \% "$, brachii $5{ }^{\prime \prime \prime}$, antibrachii $4^{\prime \prime \prime}$, femoris $8^{\prime \prime \prime}$, cruris $5^{\prime \prime} 2^{\prime \prime}$, latitudo capitis immediate ante oculos sumta $3^{1} 3^{\prime \prime \prime}$, supra nares $22^{\prime \prime \prime}$; altitudo ante oculos $4^{\prime \prime \prime}$, ante nares $2 " \prime$; oculi distant a naribus $3^{\prime \prime \prime}$, a rostri apice $4^{\prime \prime \prime}$.

II abitatio el vilae ralio. IT abitat in Africa meridionali. Vitae ratio hucusque ignota.

OBSERV ATIONES. Descriplionem secundum duo Musei Zoologici Berolinensis specimina fecit. Wiegmann.

OBSERVATIO. Color flavus gulae et linearum in dorso mox in album vergit. 


\title{
GERRHONOTUS LICHENIGERUS.
}

\author{
FLE G H T N F L E C K I G E R F A L T E N B A U C H.
}

T н в. XXXIV. Fig. 2.

Character essentialis. Gastraeo albo, unicolore; notaeo olivaceo taenius duabus longitudinalibus canescentibus, maculas concolores inter se includentibus; pilei scutellis gibbis, notaei carinatis; scutorum abdominalium seriebus 12; cauda supra angulosa.

Sy N O N Mi i. Nulla.

\section{E S C R I P T I O.}

Corporis forma universalis. Caput pyramidatum, vix distinctum, vertice plano in medio subimpresso; rostrum modice longum acutiusculum fere altius quám latum, distincte angulosum, supra versus apicem subdeclive, ad latera longitudinaliter impressum; occipitis latera rotundata; mentum prorsus planum; oris rictus rectissimus infra canthum posteriorem oculorum desinens, lamina supraorbitalis distincta, operculo indistincto; canthus rostralis distinctus, rectus, rotundato-acutus; mandibula maxillam longitudine aequans; nares immediate infra finem canthi rostralis in parte posteriore scutelli sitae, subovato-rotundatae, mediocres, patulae, nargine laevi, rostri apici proximae; oculi modice magni naribus quam auribus propiores, subobliquo-horizontales; palpebra superior rudimentum per totam orbitae longitudinem expansa, inferior perfectior ope rudimenti superioris oculum in linea subobliqua claudens; aures patulae, maiusculae subtrigonae; in una eademque linea cum oris angulo sitae margine inermi; mem brana tympani. detrusa; lingua carnosa, crassiuscula, anterius attenuata medio mento affixa, lateribus et apice extimo libera, sed loco postremo dicto lingula quasi altera, attenuata, apice furcata, ultra oris apicem extensili, colore obscura insigni, exstructa; superficies linguae papillis sabtilissimis tecta; dentes maxillae quadraginta octo, approximati, subtiliter reclinati, tereticompressiusculi, anteriores conici, his posteriores parum longiores, apice subtruncati, homogenei, omnes simplices, mandibulae totidem, maxillarium magnitudine, anteriores conici, posteriores in apicis subtruncati medio absolete sinuati; dentes palatini nulli (procul dubio ut in congeneribus decidui); truncus longus, teretiusculus, aequalis, subgracilis; pectore applanato; plica in utroque latere intra aurem incipiens supra colli latera deinceps sub humero juxta pectoris abdominisque latera ducta et infra scelides prope anum excurrens, e cute abdominali sursum flexa aucta; collum longulum trunci crassitie, simplex, exclusa illa plica laterali; ca u da elongata, corpore toto, incluso capite, longior, teres, sensim in acumen strictissimum desinens, supra subverticillata, subtus glabra; pedes proportionati, posteriores anterioribus vix maiores, tereiusculi, omnes pentadactyli, digitis teretibus, gracilibus, toto liberis, unguiculatis; digiti omnes tam in maniculis quam in podariis longitudine inaequali; digitus externus podariorum a sequente lato interstitio distans, subversatilis, quartus omnium longissimus, primus brevissimus, tertius in maniculis omnium longissimus tamen parum brevior quam quartus; pori femorales prorsus nulli; ungues parvi, acutissimi, compressi, subarcuati.

Pholidosis. Pilei scutella convexa durissima, polygona, squamis dorsalibus minora, numerosa; scutellum verticale omnium maximum oblongo-hexagonum, profundius situm quam reliqua; scutellum subsequens minus subrhomboïdenm; paria scutellorum frontalium tria; scutellum rostrale minutum, fere pentagonum; scutella labiorum marginalia angusta, omnia quadrangula, planiuscula, glabra, temporum polygona, convexiuscula, omnia magnitudine subaequali; scutellum nasale parvum subpentagonum scutella marginalia labii inferioris per duplicem seriem disposita, seriei superioris his inferioris multo minora,

VVagler, АмpHib. III. 
omnia plana, glaberrima, ollongo-quadrangula; schtelhm labiale primum parvum trigonum; mentum primo in utroque latere scntellis nomnullis maioribus glaberrimis, polygonis, reliqua parte squamis laevissimis suboblique dispositis, oblongo-hexagonis, jugulum seriebns longitudinalihus decem syuamarum transverse hexagonis, magnitudine aequalibus, tectum, tamen squamae serici extinate reliquis multo minores; abdonen seriebus decen longitudinalibus squamarum rhomboïdearmm, magnitudine aequalinm; squanat: supra anm pracedentibus paullo maiores, subquadratae, onmes totins gastraci laevissimae, planissimat; squamae in caudae parts inferiore per series longitudinales dispositae, subrhomboïdeac, glaberrimae, in eins parte superiore einselem fere formae sed longitudinaliter medio fortiter compressac, unde carinatac, margine sublibero, prominulo, quare caudae superior pars subverticillata; squamac mainscnlae notaei totius ut nunc descriptae partis superioris caudae formatae, per series transversales dispositae; carina squatmarum notaci ante plican abdominalen parum snbobsoleta, in parte superiore anteriore uniuscuiusque spuantac; plica latere interno manicula et podaria parte postica granulosa-squanosa; hac parte anteriore scutellis angulosis glabris tecta; palma et planta verrucis minutis, granosis, acrodactyla scutellis pusillis, quadrangulis, lacvibus tecta.

Color. Notacum totum olivaceum; taeniae duae ex occipite supra dorsum et altra caudae finem ductae, rectissinae, viridi-canescentes, maculas concolores inter se includentes; cauda supra maculis similibus variegata; squanae nonnullae trunci lateralis hic et illic canescenti-terminatae; pedes supra, pileus et capitis latera immaculata, corpus totum subtus, inclusa cauda, alba, unicolor, nitore vitreo; lingua incarnata, excepto eius appice obscuro; irides...?

Proportio corporis partium e mensura. Longitudo a rostri apice ad caudae finem 115\%", caudae $7 \% "$, capitis $10 \%{ }^{\prime \prime \prime}$, humeri $6^{\prime \prime \prime}$, antibrachii $4^{\prime \prime \prime}$, femorum $6{ }^{1 / 2}{ }^{\prime \prime \prime}$, tibiae $5^{\prime \prime \prime}$; ambitus capitis ad aures 2", trunci in medio et prope axillas et ante femora $2 "$.

Habitatio et vitae ratio. Habitat in Mexico, in quercetis inter saxa. Gerrlonoti vivos pullos pariunt.

OBSERVATIONES. Specimen quod descripsi et depingi curavi recentissime necalum, nitidissimum, misit e Mexico ad Museum Monacense dom. baro DE KARWANsKX. Intra anni spatium in spiritu vini servalum colores non mulavit. Genus, ,Gerrhonotus" cum speciebus sex ii peculiaribus, descripsil amicissimas Dr. el Prof. TIEGMANN in OKENIT Iside (Vol. XXI.p. 379 caet.) An ad speciem nostram HERNANDEZH, "YZTACTECoIZIN, seu alba lacerta" (cap. XIX), sive eius ,TLILCUETZPALLIN, sell nigra lacerta" (cap. XXXV) referenda? Sed utriusque color principalis ad Gerrhonolum lichenigerum non optime yuadrat. 



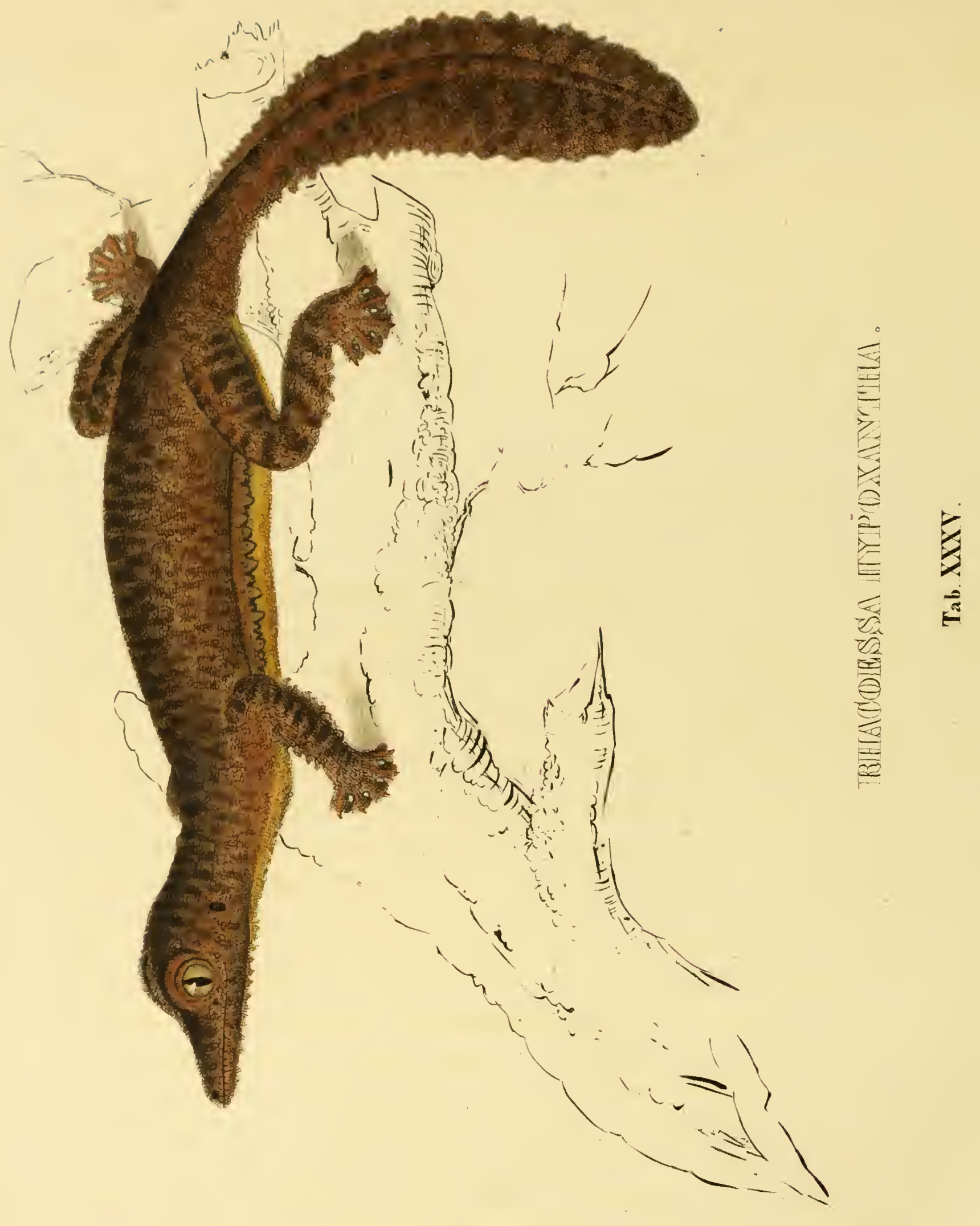




\title{
RHACOESSA HYPOXANTHA.
}

\author{
G E L B B A U C H I G E R F R A N Z F N G E C K O.
}

T A B. XXXV.

\begin{abstract}
Character essentialis. Notaeo sordide fusco, maculis obscurioribus inter se connexis reticulato-variegato; pedibus obscurius fasciatis; gastraeo nitide flavo, immaculato.
\end{abstract}

Synonyia. Fa mo-Cantratra, Flaccourt Hist. de Madagasc. 1661. Chap. 38. p. 155, Dapper Descript. de l'Afrique p. 458. La Tête-plate LAcép. Hist. nat. des Quadr. ovip. T. I. p. 325. tah. 30. (fig mediocr.) Dieselbe übersetzt v. Bechst. Bd. 2. p. 108. t. 14. f. 2. (Plattköpfiger Gecko.) Bonvat. Erpet. sy. 11. 52. pl. 11 f. 2. Stellio fimbriatus Schnein. Amphib. Pliysiol. Spec. II. p. 31. 11. 10. Lacerta fimbriata Donnd. Zool. Beitr. III. S. 134. n. 8. Siraw Gen. Zool. I1I. p. 112. Gecko fimbriatus Latr. Hist. nat. des Rept. T. II. p. 54. Fig. 4. Daud. Rept. IV. p. 160. t. 52. Mrrr. Syst. Amph. p. 40. - Bosc Nouv. Dict. d'hist. nat. XII. p. 513. tab. D. 30. Le Gecko frangé Cur. R. an. II. p. 55. - Wagl. Natïrl. Syst. d. Ampl. p. 142.

\section{E S C R I P T I O.}

Corporis forma universalis. Caput indistinctum a collo, elongatum, esocinum, rostro longissimo, admodum depresso, lato, apice rotundato, versus frontem altiorem sensim adscendente; vertex ipse planus, latus; occipitis latera nonnihil rotundata; canthus rostralis prorsus nullus; maxillae apex extimus subdeclivis, cum apice mandibulae aecquali longitudine; oris rictus longus, paullo pone et infra canthum posteriorem oculorum desinens, labiorum margine rectissimo; mandibula humillima; mentum applanatum; nares rostri apici admodum approximatae laterales, parvae, rotundae, margine simplici, superiore prominulo; oculi maximi, circulares, admodum protuberantes, totam fere altitudinem laterum occupantes, auribus quam naribus approximatiores, pupilla verticali-elliptica; palpebra inferior prorsus nulla, superioris rudimentum in forma plicae arcuatae intra orbitae marginem et bulbum oculi reflexae; a $\mathrm{res}$ distinctae pone oris angulum sitae, parvae, meatu verticali, nargine simplici; membrana tympani detrusa; lingua lata, carnosa, plana, apice extimo libera ibidemque subexcisa; dentes coelorhizi, tomiorum lateri interno agglutinati, homogenei, numerosissimi, oppositi, recti, erecti, simplices, cylindracei, unicuspides, corona indumento vitreo obducta, vix acutata, compressiuscula; posteriores longitudine sensin decrescentes, laniarii et palatini nulli; truncus longulus, robustus, supra convexus, subtus planus, undique subaequali latitudine; collum longulum trunci forma, lıoc parum angustius; cutis libera, pendula, margine fimbriata utrinque a rostri apice infra mandibulan supra colli ac trunci latera inferiora ducta, fine abdominis desinens: limbus cutaneus pendula similis in utroque margine pedum, tam anticorum quan posticorum; cauda circiter trunci longitudine, tereti-depressa, utrinque ad latus cute laxissima, integra, attamen extimo margine fimbriata, versus caudae basin magis magisque attenuata, limbata, unde quasi formant gubernaculi apice spatuliformi repraesentans: pedes validi, depressiusculi: anteriores posterioribus multo minores, omnes pentadactyli; digiti basi membrana connexi, reliqua parte fissi, omnes unguculati et in utroque latere cute squamulosa, margine integra, apice rectiusculo-truncata large limbati, apice pro recipiendo unque fissi: lyypodactyla foliolis cutaneis erectis numerosis, transversim dispositis obtecta, pori femorales distincti: ungues compressi, arcuati, acutissimi.

Pholidosis. Caput, collum, truncus, cauda et pedes supra squamis minimis, granosis, interjectis hic et illic paullo maioribus, labiorum margo scutellis, quadrangularibus, planis, magnitudine aequalibus, utrinque per seriem unicam dispositis, gastraeum totum squamis nonnihil maioribus, rhomboïdalibus, laevibus, planis, homogeneis obtecta.

Waglek, АMPHib. III. 
Color. Notaeum totum sordide fuscum, maculis obscurioribus, inter se connexis variegatum; pedes obscurius subfasciati: gastracum totum nitide flavum, immaculatum: irides ...?

Proportio corporis partium e mensura. Longitudo a rostri apice ad caudae finem 10", caudae $4 \% "$.

Habitatio et vitae ratio. Habitat in insula Madagascar (et ad flumen Senegal, fide Adanso n.) Degit supra arborum corticem, egregie salit a rano ad ranum, cute laxa, pendula pedum, trunci et caudae adminiculante: agiliter scandit sed tarde humo incedit: noctu victum quaerit interdiu in arborum cavis latitans, victitat insectis: dorsi sed non abdominis colorem pro lubitu mutat; audit Famocantratra apud Madagascarienses, i. e. bestia versus pectus saliens. Ob dorsi coloren fuscum a cortice arborum, cui adhaeret, difficillime distinguenda.

OBSERVATIONES. Lutetïs Parisiorum vidi et descripsi nonnulla specimina nitidissima; ynatuor sive yuinque servat Museum Parisiense. Specimen nuper captum cel. OpPEL coloribus adumbravit; idem nostra icon repraesentat. Speciminum per longum tempus in spiritu vini conditis gastraeum albicat, notaum in griseum vergit. 



$$
k
$$




\title{
TRACHYSAURUS PERONII.
}

\author{
$\begin{array}{llllllllllllllllll}\mathrm{P} & \mathrm{E} & \mathrm{R} & \mathrm{O} & \mathrm{N} & \mathrm{S} & \mathrm{K} & \mathrm{R} & \mathrm{U} & \mathrm{S} & \mathrm{T} & \mathrm{E} & \mathrm{N} & \mathrm{S} & \mathrm{C} & \mathrm{I} & \mathrm{N} & \mathrm{K}\end{array}$
}

\section{T н в. XXXVI.}

Character essentialis. Castaneo-fuscus, lateribus obsolete obscurius fasciatis, subtus dilutior; notaeo squamis granuloso-asperulis.

Symonym. Scincus Peronii Duneril in Mus. Paris. Trachysaurus rugosus Gray in Phil. Kings Narrative of a Survey of the coasts of Australia. London 1827. Vol. II. Append. p. 430. WAG L. Natürl. Syst. d. Amph. p. 163.

\section{E S C R I P T T O.}

Corporis forma universalis. Capuı admodum distinctum, magnum, tetragono-pyramydale, pileo plano, occipitis lateribus rotundato-protuberantibus, rostro modice longo; nares a rostri apice interstitio brevissimo distantes in medio scutelli sitae, mediocres, subrotundae, patulae, simplices; oculi nares inter et aures intermedii, pupilla circulari; lamina supraorbitalis distincta; aures supra oris angulum sitas, angustae, verticali-oblongae; dentes breves, crassi, conici, in palato nulli; truncus longulus, crassus, subaequalis, tereti-depressiusculus; collum breve occipite multo angustius, absque torque squamarum, prorsus simplex; cauda brevis, circiter dimidium parten corporis longitudine aequans, crassa, integra, depresso-conica; pedes quatuor perfecti, ambulatorii, pentadactyli, crassi, proportionati; antici posticis debiliores; digiti distincti, fissi, breviusculi, validi, teretes, omnes unguiculis exstructi, laterales longitudine sensim decrescentes; digitus plantae quintus a quarto brevi interstitio remotus; pori femorales prorsus nulli; ungues breves, validi, subarcuato-conici.

Pholidosis. Pholidosis notaei dura, subossea, gastraei ordinaria, membranacea; caput, collum, truncus et cauda supra scutellis homogeneis (per fascias obliquas dispositis) rhomboïdalibus, convexis, carina convexa, subspinosa auctis, epidermide granulosa obductis, subtus scutellis largis, hexagonis, imbricatis, glabris, homogeneis tecta; scutella ad latera capitis et in huius parte inferiore scutellis verticalibus maiora.

Color. Caput, collum, truncus, cauda et pedes supra castaneo-fusca, subtus dilutiora; trunci latera fasciis obscurioribus parum distinctis obsita; ungues corneo-fuscescentes; irides ...?

Proportio corporis partium e mensura. Longitudo (speciminis in Museo Britannico) 14", capitis $3^{\prime \prime}$, caudae $4^{\prime \prime}$, trunci 7".

Habitatio et vitae ratio. Habitat in Nova-Hollandia, in Freto "King George the Third's Sound" dicto.

OBSERVATIONES. Animal hoc pholidoseos suae struclura admodum insigne a celeberrimo PERON de. tectum, nuper a cl. KrNe in Novae-Hollandiae freto supra dicto repertum est. Specimen, cauda laesum, possidet Musenm Parisiense et Londinense, tertium servat Museum Societatis Linneanae Londinensis. Picturam, a nobis datam cel. OPPEL Lntetiis Parisiorum elaboravit. 





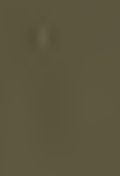

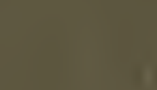

in.

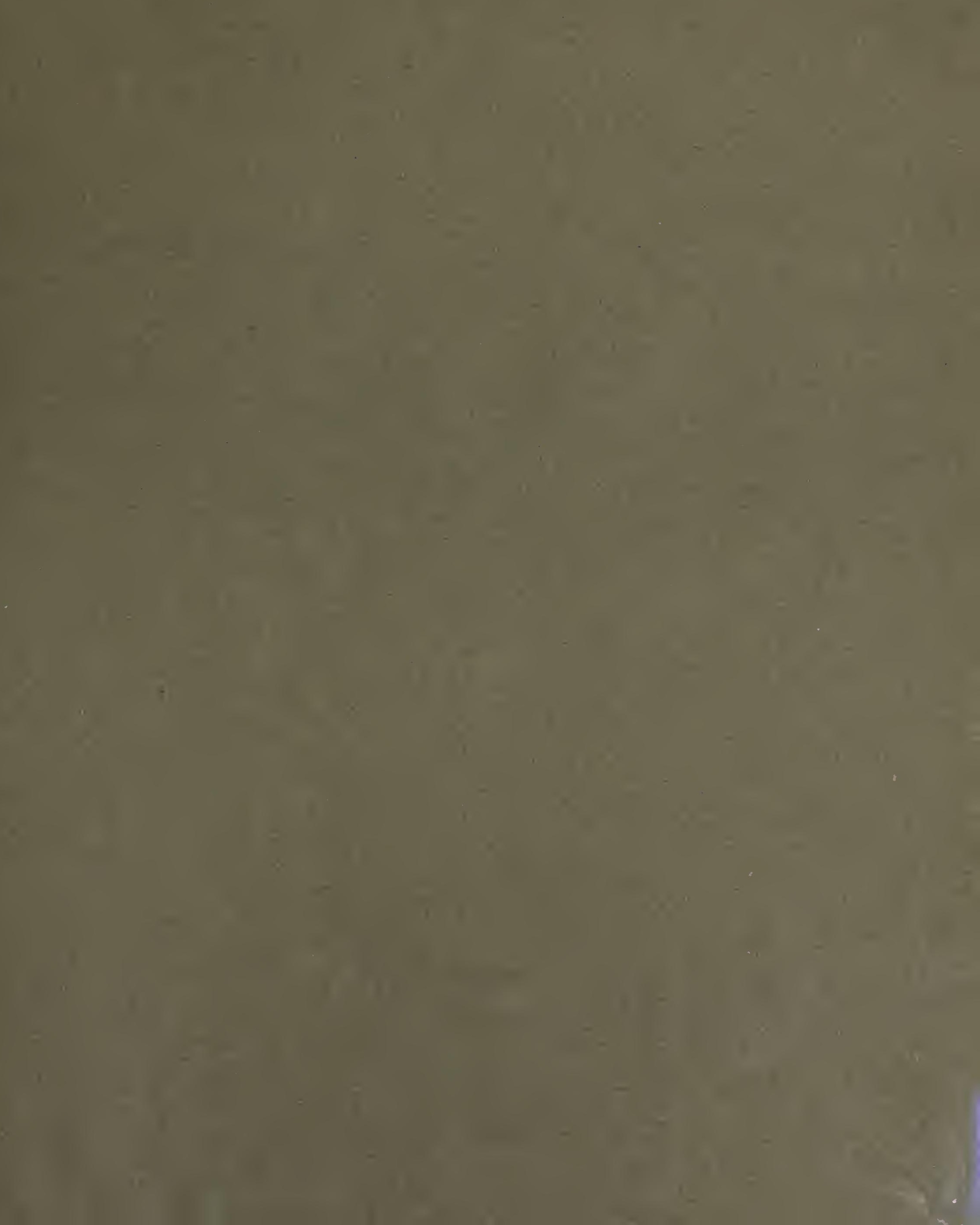


? 



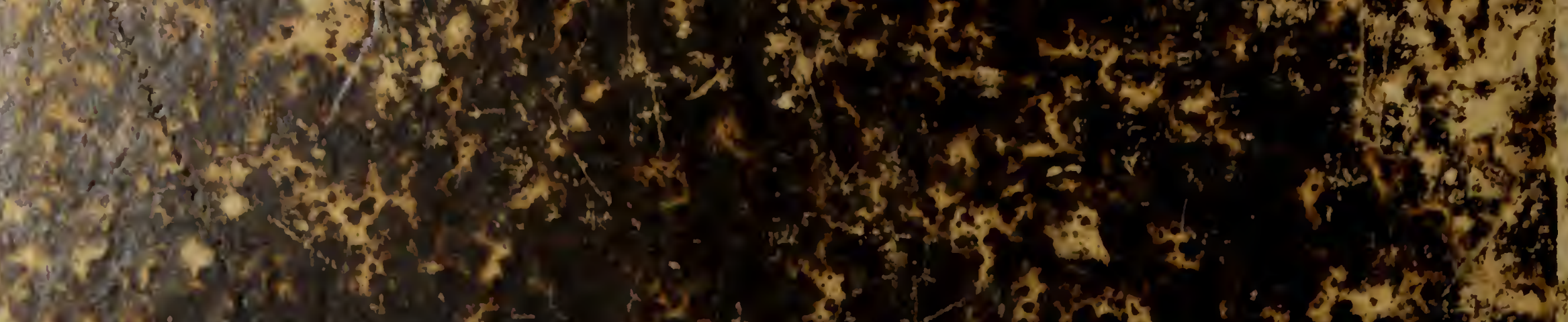

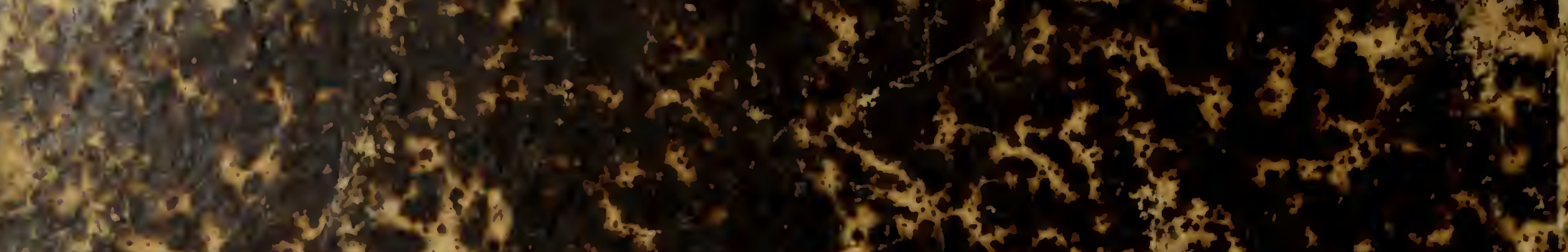

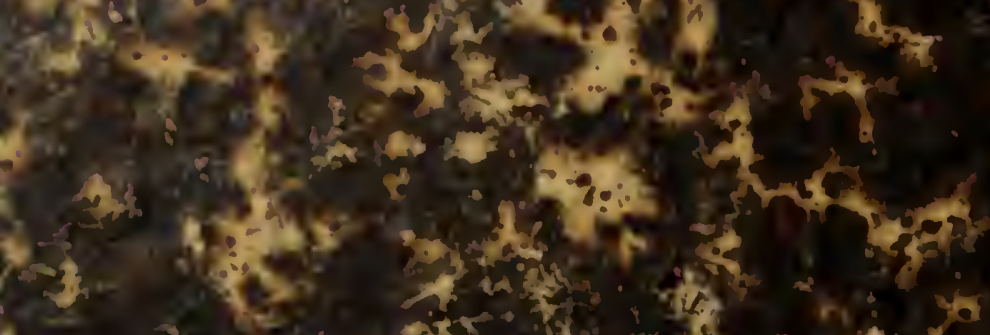

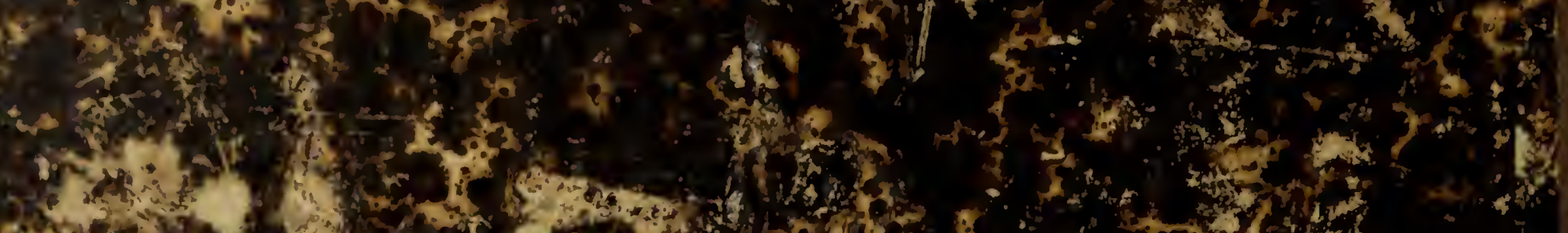

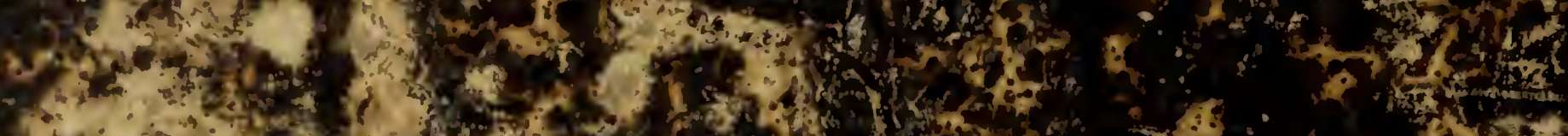

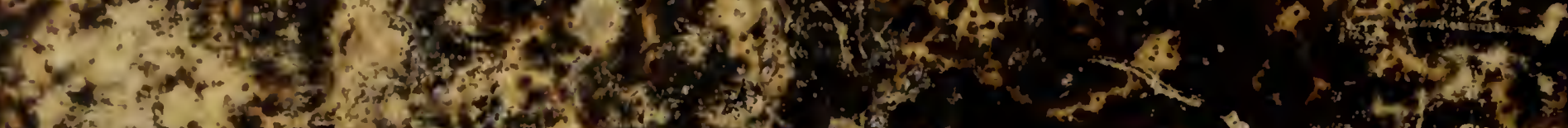
H.

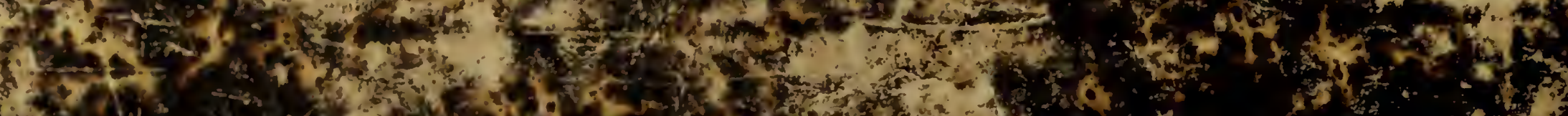

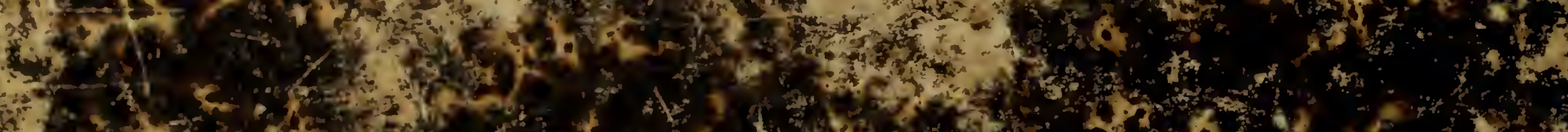

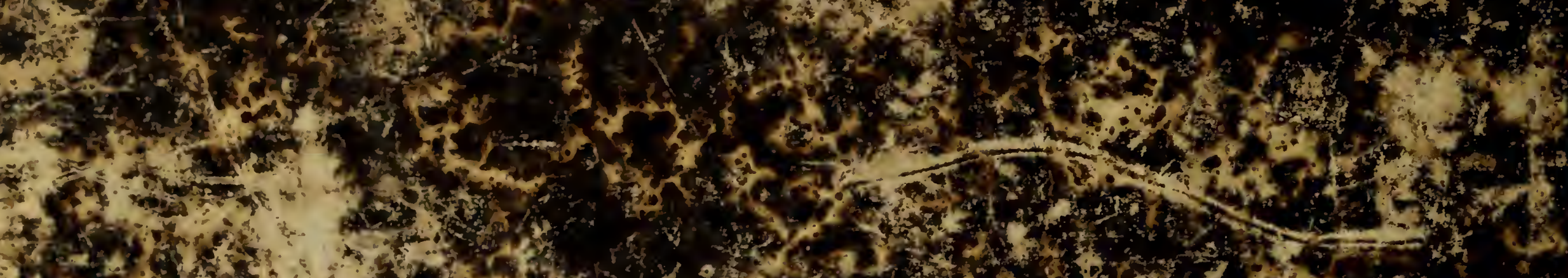
1.2. n)

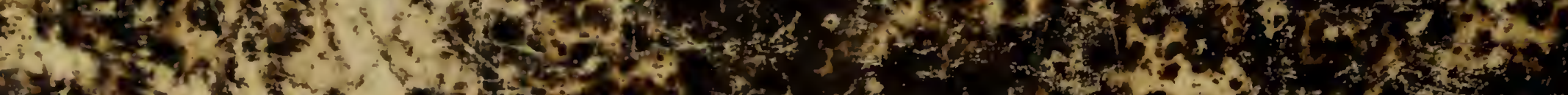
wo

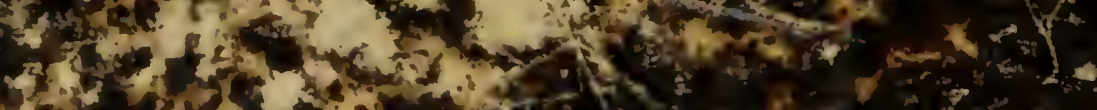

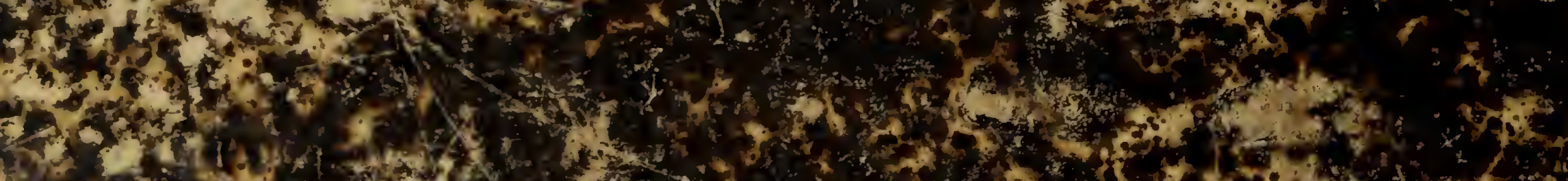

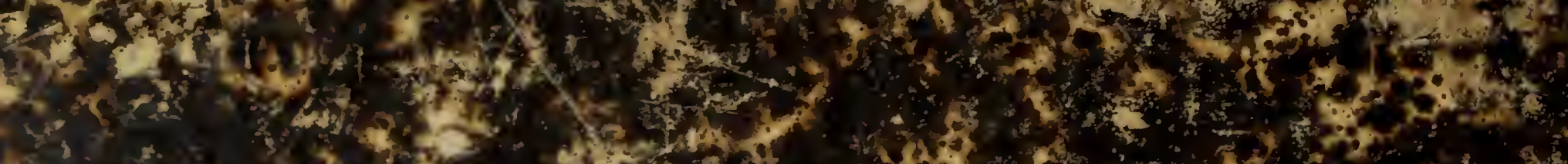
$x_{x \rightarrow 3}+4+2$

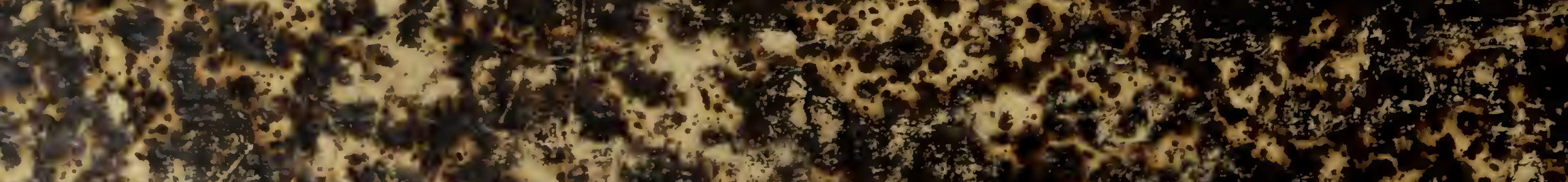
1. C.

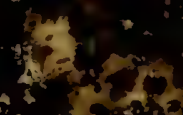

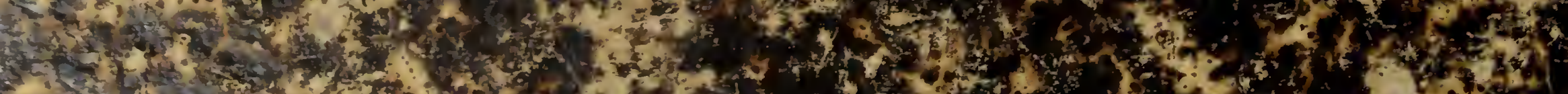

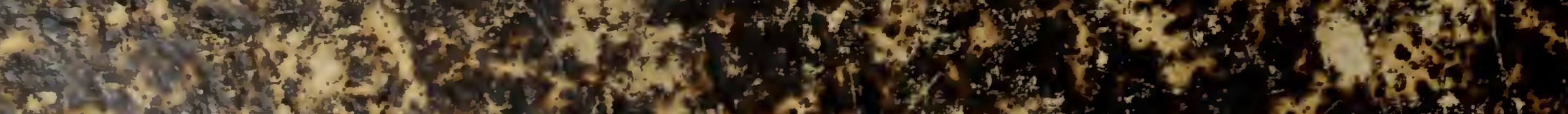

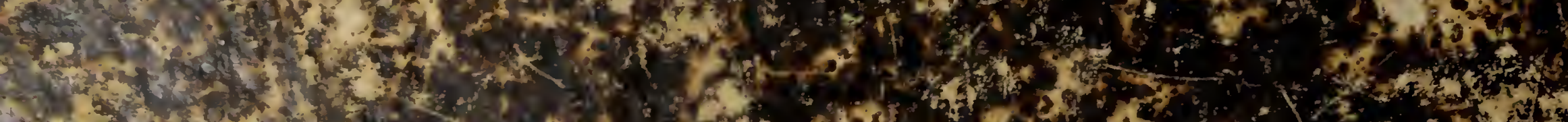

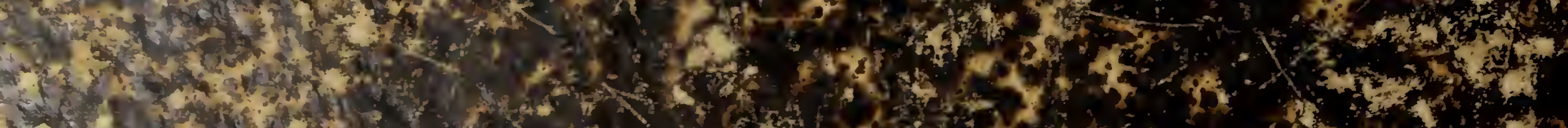

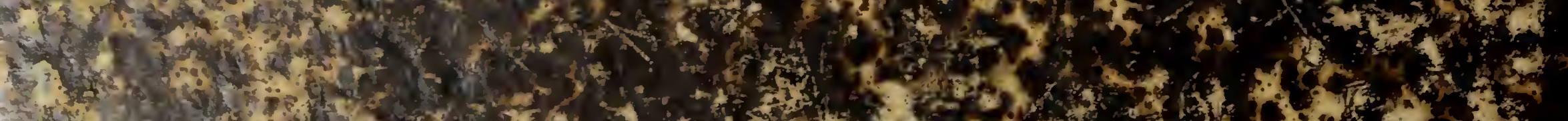

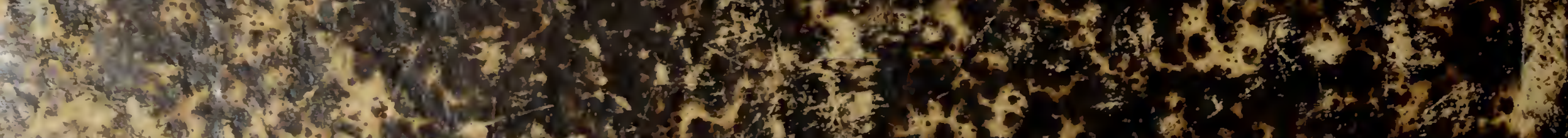

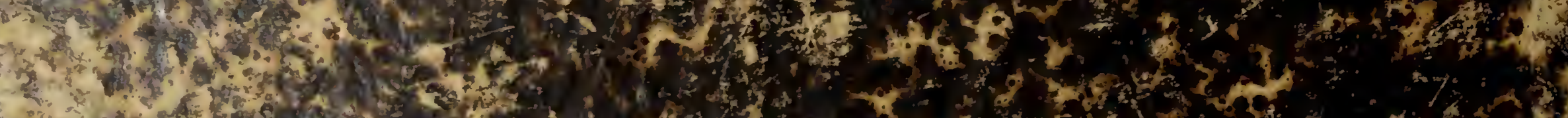
(1)

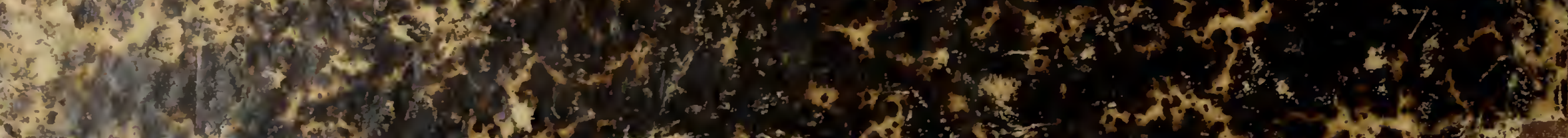

